UNIVERSITE DU QUEBEC

\author{
MEMOIRE \\ PRESENTE A \\ L'UNIVERSITE DU QUEBEC A CHICOUTIMI \\ COMME EXIGENCE PARTIELLE \\ DE LA MAITRISE EN RESSOURCES ET SYSTEMES
}

\author{
PAR \\ LINDA GELEYN \\ B.SC.
}

\title{
MODELISATION D'UN SYSTEME GENERAL DE GESTION INTELLIGEMMENT ASSISTE PAR ORDINATEUR (EIAO)
}

Application à l'enseignement de l'algèbre de Boole et à la gestion d'un bras manipulateur(robotique)

MAI 1992 


\section{Bibliothèque}

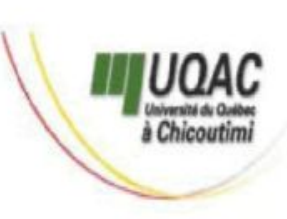

\section{Mise en garde/Advice}

Afin de rendre accessible au plus Motivated by a desire to make the grand nombre le résultat des results of its graduate students' travaux de recherche menés par ses research accessible to all, and in étudiants gradués et dans l'esprit des accordance with the rules règles qui régissent le dépôt et la governing the acceptation and diffusion des mémoires et thèses diffusion of dissertations and produits dans cette Institution, theses in this Institution, the I'Université du Québec à Université du Québec à Chicoutimi (UQAC) est fière de Chicoutimi (UQAC) is proud to rendre accessible une version make a complete version of this complète et gratuite de cette œuvre. work available at no cost to the reader.

L'auteur conserve néanmoins la The author retains ownership of the propriété du droit d'auteur qui copyright of this dissertation or protège ce mémoire ou cette thèse. thesis. Neither the dissertation or Ni le mémoire ou la thèse ni des thesis, nor substantial extracts from extraits substantiels de ceux-ci ne it, may be printed or otherwise peuvent être imprimés ou autrement reproduced without the author's reproduits sans son autorisation. permission. 


\section{A Ghislain, Marie-Josée, Jean-François et Michel.}




\section{RESUME}

La présente recherche aborde la modélisation d'un système général de gestion de ressources (humaines ou non) agissant en temps réel dans des situations de transformations successives des dites ressources. Pour illustrer les concepts nous avons retenus deux situations de gestions: l'une concerne la modélisation de l'apprentissage d'un sujet de l'algèbre de Boole, l'autre concerne la modélisation de la manipulation d'un bras mécanique en robotique. Ces deux exemples permettent également de démontrer l'applicabilité du modèle préconisé.

Un tel système automatique doit posséder certaines qualités essentielles pour être efficace: être "intelligent", évolutif et adaptatif. Celles-ci sont fixées à partir de modèles tirés du domaine de l'éducation et de systèmes tutoriels existants dans ce dernier. Une classification des systèmes tutoriels intelligents permet de situer le nôtre dans la catégorie des systèmes tutoriels intelligents et adaptatifs.

On cherche par la suite à concevoir les composantes intelligentes du système en se basant sur un modèle pédagogique pour la gestion d'une situation d'apprentissage (Ouellet [37]). On obtient ainsi un modèle tutoriel de gestion impliquant quatre catégories d'éléments (règles et faits): programme, contexte, activité et résultat. La structure générale des règles de gestion et des formes des contenus de ces bases de connaissances est ensuite présentée. Elle permet au système de résoudre tant qu'il le peut, et ce de façon automatique, la gestion des événements en adaptant continuellement les normes de décision basées sur la compatibilité des faits. Lorsqu'il ne peut trouver la solution par lui-même, le système fait appel à l'externe pour que l'expert concerné ajoute les éléments manquants dans ses bases de connaissances.

Cette structure est concrétisée dans un exemple concernant l'apprentissage d'un sujet emprunté à l'algèbre de Boole puis implantée sur micro-ordinateur. Cette implantation du système implique la définition et la gestion explicite de tous les 
éléments nécessaires à la bonne marche du système. Lors de simulations de ce programme(PROLIN), l'obtention de résultats conformes à ce qui était espérés démontre l'efficacité du système.

Un deuxième exemple d'application concernant la gestion d'un bras manipulateur est présenté. Ce dernier n'a pas été programmé mais tous les éléments nécessaires à cette programmation sont présentés. Cet exemple permet de démontrer la généralisation du système à des situations autres que l'enseignement. 


\section{REMERCIEMENTS}

Un travail tel que celui-ci demandant une bonne préparation ainsi qu'un apport important de connaissances et de techniques, je me suis entourée de personnes compétentes qui m'ont beaucoup aidé. Je tiens donc à remercier:

- Jean-Joël Vonarburg (directeur de recherche) pour avoir eu la patience de lire et relire mes textes sous un oeil différent me permettant ainsi d'acquérir une meilleure perception de ce que doit être un mémoire de maîtrise.

- Richard Tremblay (co-directeur de recherche) pour avoir superviser le cheminement de cette recherche et ce, du tout début à la toute fin.

- Carl Houde (programmeur analyste au laboratoire REIB-UQAC en 1989-1990) pour m'avoir montré les rudiments de la programmation avec ARITY-PROLOG en plus de me prodiguer de nombreux conseils pratiques à ce sujet.

- Ghislain Goyette (ingénieur et époux) pour avoir fait tous les schémas se trouvant dans ce mémoire en plus de m'éclairer au sujet des nombreux utilitaires du logiciel de traitement de texte utilisé pour le rédiger.

Merci donc à toutes ces personnes. 
TABLE DES MATIERES

INTRODUCTION

\section{CHAPITRE I L'ENSEIGNEMENT COMME GESTION DES}

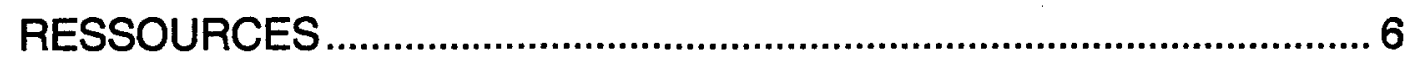

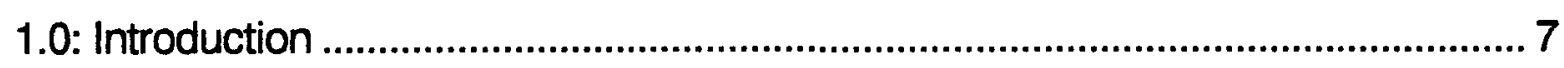

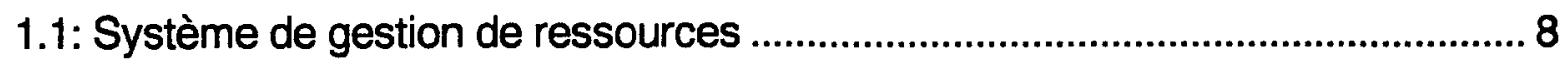

1.2: Première définition du modèle .................................................................... 9

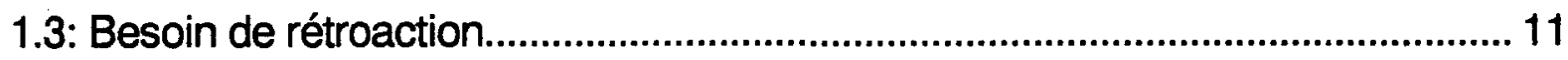

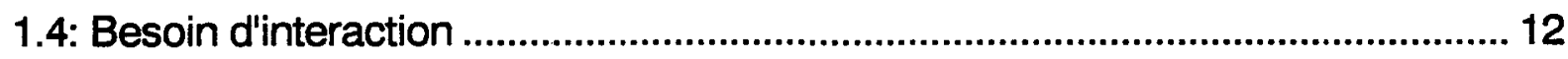

1.5: Besoin d'adaptabilité ........................................................................................ 13

1.6: Besoin de plusieurs niveaux d'évaluation ......................................................... 14

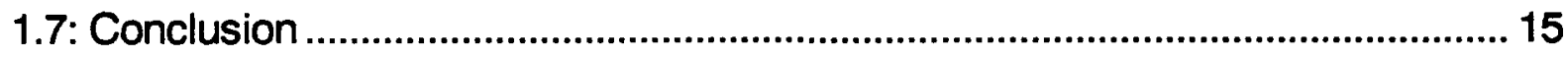

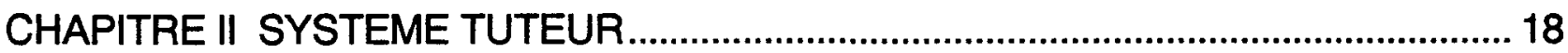

2.0: Les différents systèmes tuteurs ........................................................................... 19

2.0.1: Le modèle étudiant................................................................................... 19

2.0.2: Système tutoriel conventionnel ................................................................. 20

2.0.3: Système tutoriel intelligent.......................................................................... 20

2.0.4: Système tutoriel adaptatif ......................................................................... 21

2.1: Le modèle étudiant ................................................................................................. 21

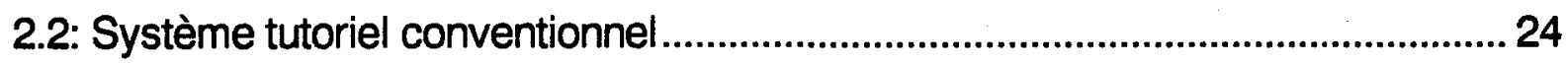

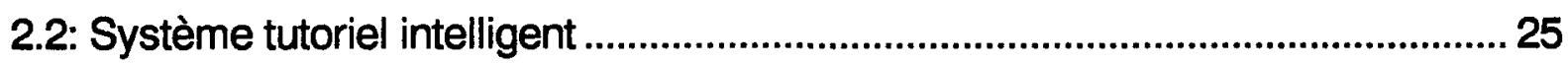

2.2.1: Un exemple de système tutoriel intelligent: GUIDON............................. 26

2.4: Système tutoriel intelligent et adaptatif.............................................................27

2.5: Recherche d'une nouvelle structure minimale ..................................................29

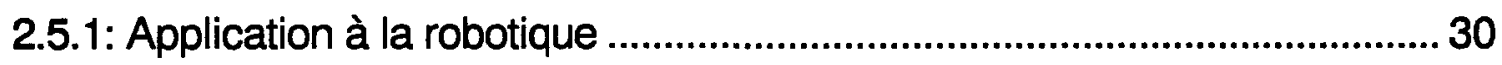

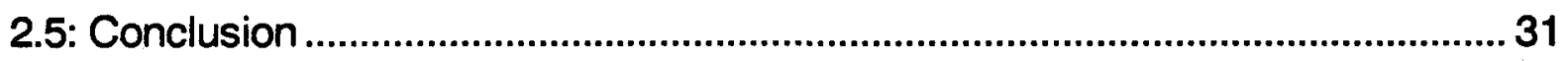


CHAPITRE III LE MODELE UTILISE

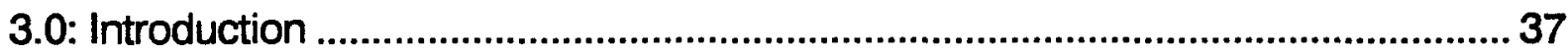

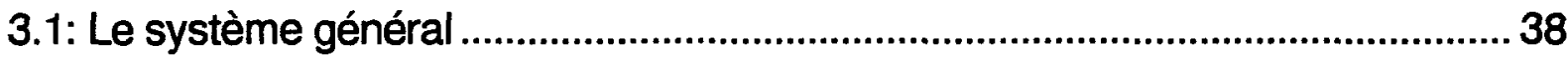

3.1.1: Le modèle de Le Moigne ........................................................................... 38

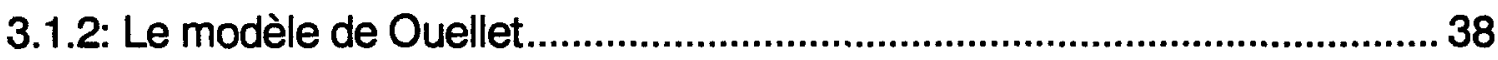

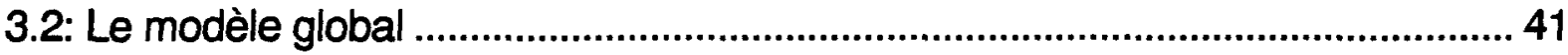

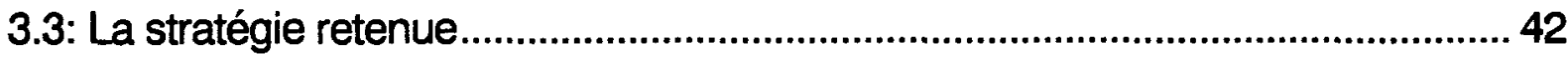

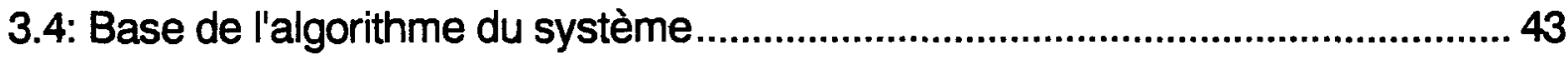

3.5: Généralisation du modèle .................................................................................. 47

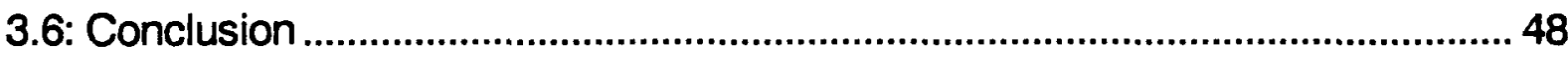

CHAPITRE IV LES REGLES DE GESTION

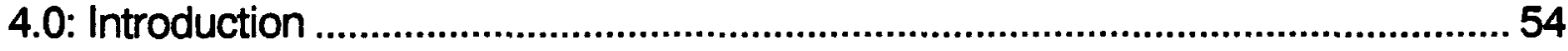

4.1: Référentiel de Le Moigne (Référentiel TEF) ..................................................... 55

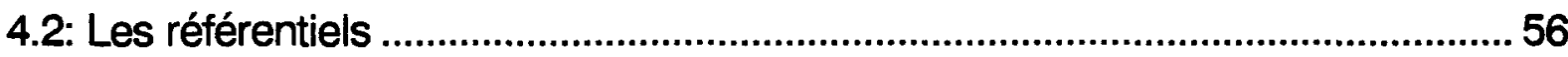

4.3: Degrés de pertinence et normes de compatibilité .............................................58

4.3.1: Degrés de pertinence.............................................................................. 58

4.3.2: Normes de compatibilité ........................................................................... 59

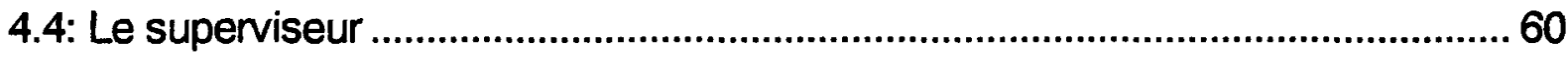

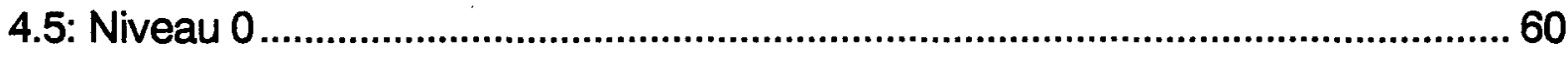

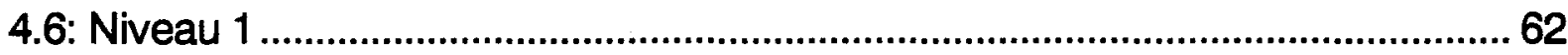

4.6.1: Système avec bases d'importances inégales ........................................ 65

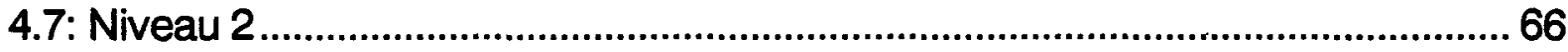

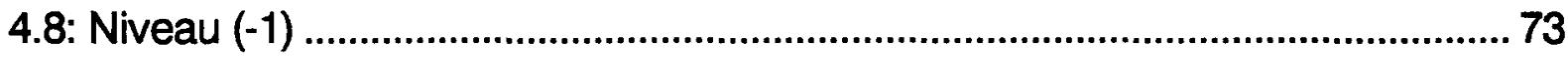

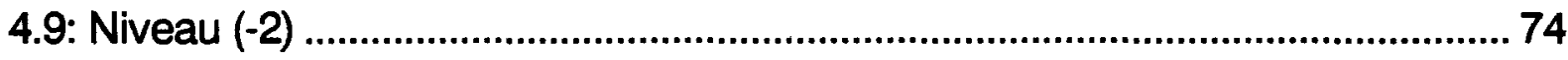

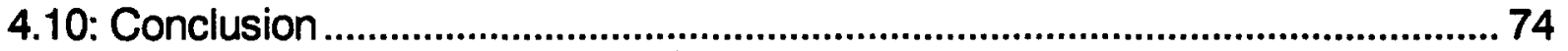


CHAPITRE V STRUCTURATION DES BASES DE CONNAISSANCES. .79

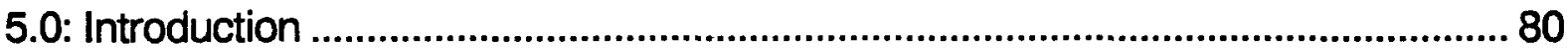

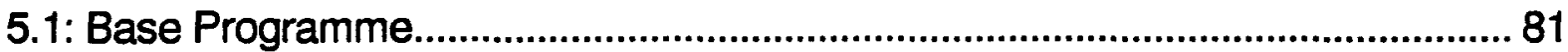

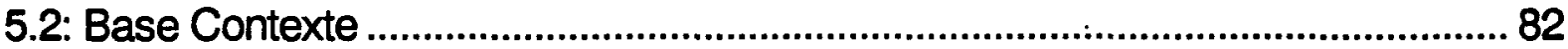

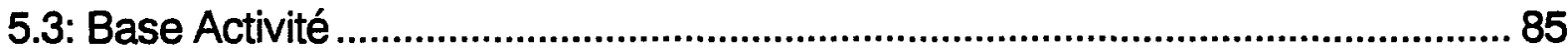

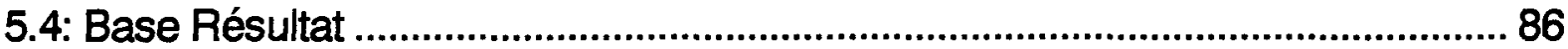

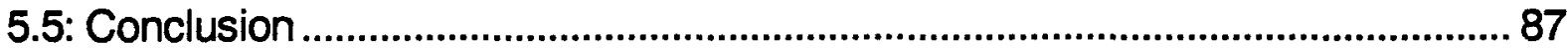

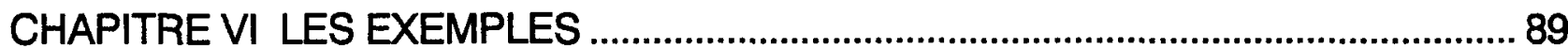

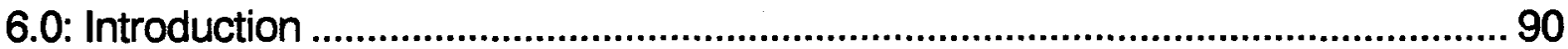

6.1: L'apprentissage de l'algèbre de Boole (programme PROLIN)......................... 91

6.1.1: Théorèmes de base de l'algèbre booléenne ............................................ 91

6.1.2: Les bases de connaissances ..................................................................94

6.1.3: Programme PROLN................................................................................ 98

6.2: La gestion d'un bras manipulateur.....................................................................99

6.2.1: Présentation de l'exemple ....................................................................99

6.2.2: Les bases de connaissances ............................................................... 100

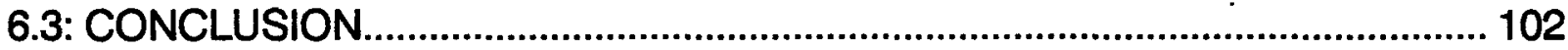

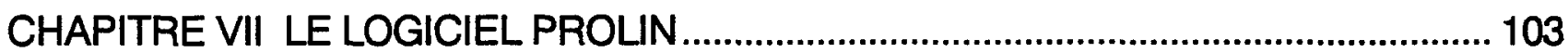

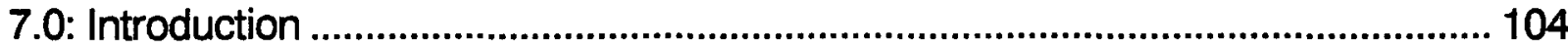

7.1: Analyse fonctionnelle du programme PROLIN................................................. 105

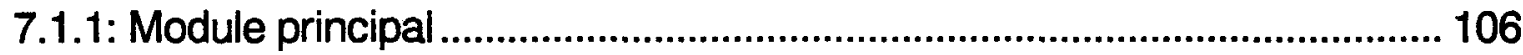

7.1.2: Module de préparation de la base de connaissances.............................. 106

7.1.3: Module global (Modèle adaptatif) ............................................................ 108

7.1.4: Module d'initialisation du système......................................................... 109

7.1.5: Module de recherche du plus grand compatible .................................... 109

7.1.6: Module de recherche des faits équivalents ............................................. 110

7.1.7: Module du superviseur ............................................................................. 110

7.1.8: Modules de calcul des normes (Niveau 1) .............................................. 111

7.1.9: Modules du niveau 2 .............................................................................. 112

7.1.10: Module de vérification du niveau suivant.............................................. 113

7.1.11: Module du niveau 3............................................................................ 113 


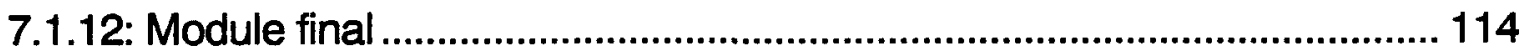

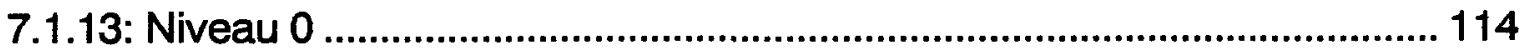

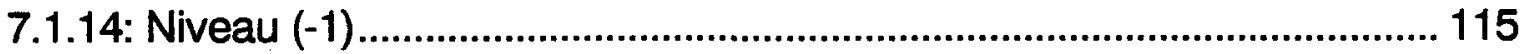

CHAPITRE VIII SIMULATIONS DU PROGRAMME PROLIN .....................................116

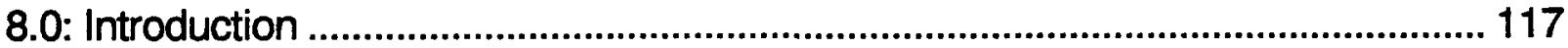

8.1: Sommaire des résultats de 4 simulations de PROLIN ..................................... 118

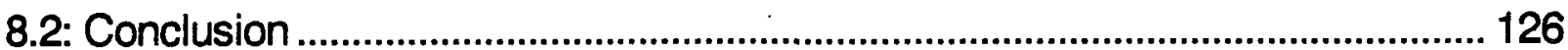

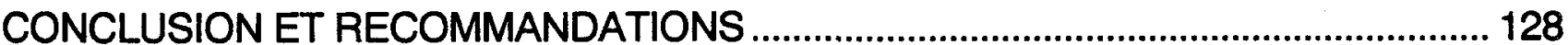

CONTENU DU RAPPORT TECHNIQUE ................................................................... 133

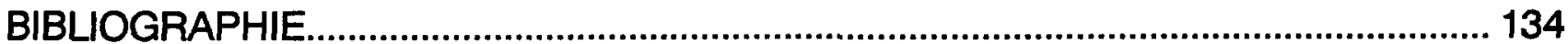

ANNEXE 1 INTEGRATION DE DEUX ROLES POSSIBLES DE L'ORDINATEUR DANS UNE SITUATION D'ENSEIGNEMENT

ANNEXE 2 SYSTEMES A TROIS BASES DE CONNAISSANCES …......................... 145

A2.1: Système avec une base dégénérée ................................................................. 146

A2.2: Système à trois bases de connaissances ...................................................... 148 


\section{LISTE DES FIGURES}

FIGURE 1.1 : Système général de transformation de l'apprenant.

FIGURE 1.2: Principales variables de la théorie de l'apprentissage

scolaire. (Bloom [04] p.22)

FIGURE 1.3 : Schéma général inspiré de Bloom

FIGURE 1.4 : Système général avec rétroaction

FIGURE 1.5: Le modèle de base de l'apprentissage et de la mémorisation qui sous-tend les théories modernes du traitement de l'information. (Gagné [17] p.15)

FIGURE 1.6: Incorporation du modèle de Gagné dans le modèle général.

FIGURE 2.1 : Principaux systèmes tutoriels

FIGURE 2.2 : Connaissance comme sous-ensemble de celle d'un expert

(Kass [26]).

FIGURE 2.3 : Apprentissage comme recouvrement de la connaissance espérée (Kass [26])

FIGURE 2.4 : Distorsions (Kass [26]).

FIGURE 2.5 : Structure de simulation d'enseignement (inspiré de Smith

et Blandford [51] p.53)

FIGURE 2.6 : Système tutoriel intelligent (Fugère, Geleyn Tremblay [16]) .26

FIGURE 2.7 : Structure générale de GUIDON (Clancey [10] p.5) 26

FIGURE 2.8: Transfert d'expertise: apprentissage, conseil et enseignement (Clancey [10] p.9)

FIGURE 2.9 : Tuteur d'enseignement (Fugère, Geleyn, Tremblay [16])

FIGURE 2.10: Système tuteur à deux niveaux pour la gestion d'une situation d'apprentissage. (Inspiré de Lewis [30])

FIGURE 2.11: Système tuteur à deux niveaux pour la gestion d'un robot. (Inspiré de Lewis [30])

FIGURE 2.12: Tuteur gestionnaire d'une situation d'apprentissage

FIGURE 3.1: Système général de Le Moigne ([28] p.34). 
FIGURE 3.2: Organisation des données de l'intrant, de la transformation

$$
\text { et de l'extrant (Ouellet [37] p.103) }
$$

FIGURE 3.3: Une situation d'apprentissage avec quatre ensembles de

$$
\text { variables du système scolaire (Ouellet [37] p.101). }
$$

FIGURE 3.4 : Structuration des règles pour un système à 4 bases.

FIGURE 3.5: Le cycle du comportement et de l'apprentissage (Woodruff

[53] p.3)

FIGURE 3.6 : Mode de progression 43

FIGURE 3.7: Schéma fonctionnel des niveaux 0 et 1 du système. 45

FIGURE 3.8 : La chenille ou schéma fonctionnel du système global.. 46

FIGURE 3.9 : Structuration des règles pour un système à 3 bases. 48

FIGURE 4.1 : Référentiel TEF (Temps, Espace, Forme) permettant de

repérer la position des objets soumis à des processus.

(Le Moigne [28] p.63). 56

FIGURE 4.2 : Sous-référentiel PCA (Programme-Contexte-Activité) 56

FIGURE 4.3: Structuration des règles pour un système à 4 bases .57

FIGURE 4.4 : Superviseur. 60

FIGURE 4.5 : Structuration des règles. 61

FIGURE 4.6 : Niveau 1 de règles 62

FIGURE 4.7 : |ai| comme longueur du longueur résultant 63

FIGURE 4.8: Retour nécessaire si on désire modifier la norme de |ai préc|.

FIGURE 4.9: Retour nécessaire pour faire un nouveau choix de programme

FIGURE 4.10: Retour nécessaire pour faire un nouveau choix de contexte. 69

FIGURE 4.11: Niveau 2 69

FIGURE 4.12: La trace lors du passage au niveau 2 .72

FIGURE 5.1 : Structuration de la base Programme 81

FIGURE 5.2 : Structuration de la base Contexte 83

FIGURE 5.3 : Structuration de la base Activité 85

FIGURE 5.4 : Schéma fonctionnel du système autour de la base Activité 85

FIGURE 5.5 : Structuration de la base Résultat 86 
FIGURE 5.6 : Figure hiérarchique de la formation d'un concept (Woodruff

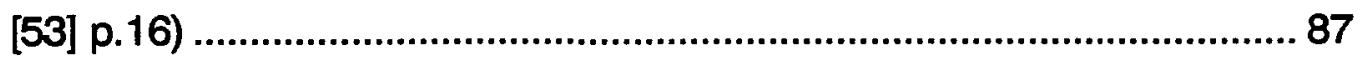

FIGURE 6.1 : Programme (Algèbre de Boole) ............................................................... 94

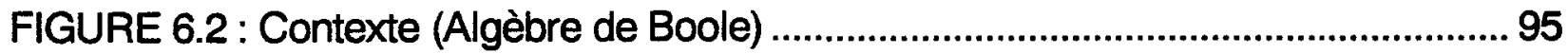

FIGURE 6.3 : Activité (Algèbre de Boole) .........................................................................96

FIGURE 6.4 : Résultats (Algèbre de Boole) ................................................................. 97

FIGURE 6.5 : Programme (Bras manipulateur) ................................................................. 100

FIGURE 6.6 : Activité (Bras manipulateur) ..................................................................... 101

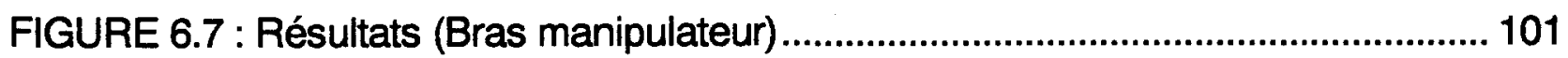

FIGURE 7.1 : Schéma fonctionnel du programme PROLIN........................................... 105

FIGURE A1.1: Ordinateur jouant le rôle de l'enseignent didacticien (Lewis [81] p.43)

FIGURE A1.2: Ordinateur comme ressource parallèle d'investigation (Lewis [30] p.43)

FIGURE A1.3: Système tuteur à deux niveaux pour la gestion d'une situation d'apprentissage.

FIGURE A2.1: Système à trois bases de connaissances. 
INTRODUCTION 


\section{INTRODUCTION}

La manipulation d'un robot, le déroulement d'une situation d'apprentissage ou tout autre projet comme la construction d'un pont, la mise en marche d'une entreprise, etc... sont des champs disciplinaires où la gestion joue un rôle capital. Une modélisation d'un système de gestion général de ressources (humaines ou non) permettrait de traiter plusieurs aspects fonctionnels de ces situations de gestion. Ce modèle théorique nous conduira à un système informatique utilisable dans plusieurs domaines. Le système visé devra tenir compte, à son plus haut degré d'abstraction, de situations de gestion de ressources humaines, c'est pourquoi il devra simuler un comportement intelligent à l'aide de règles, ce qui le rendra plus adaptable. Le but de la présente recherche est donc de produire un système informatique de gestion générale de ressources (humaines ou non) simulant un comportement humain.

Comme stratégie, il ne s'agit pas d'explorer de façon exhaustive toutes les situations spécifiques de gestion et ce, dans chacun des domaines d'applications possibles, il s'agit plutôt d'essayer de dégager des règles de décision et d'action qui soient communes à un maximum de situations et un maximum de domaines d'application. Le système projeté pourra s'appliquer à plusieurs domaines tels:

-la gestion des mouvements d'un robot,

-la gestion d'une partie d'échec,

-la gestion de capitaux,

-la gestion d'une situation d'apprentissage,

- la gestion d'un projet de construction,

-la gestion d'un problème quelconque, -etc. 
Ces situations de gestion contiennent suffisamment de points communs pour être traitées dans un cadre général même si ces domaines n'ont pas tous le même niveau de complexité. Parmi les situations mentionnées, nous avons choisi la gestion d'une situation d'apprentissage comme sujet d'étude car elle est la plus complexe. En effet, elle présente une situation où l'apprenant (ressource à l'entrée) est en perpétuel changement à cause du processus d'apprentissage qui est propre à chacun et de l'environnement (physique et social) qui est également fluctuant.

La tâche de l'enseignant consiste à sélectionner et à organiser les diverses influences qui entourent l'étudiant afin de promouvoir l'apprentissage (Gagné [17] p.2). C'est ce que devra faire, même à échelle réduite, le système que nous présentons. Ce dernier ne peut cependant pas remplacer l'enseignant; il n'est qu'un instrument additionnel mis à sa disposition pour l'aider dans sa tâche en lui permettant de consacrer plus de temps à l'écoute des étudiants qui manifestent le besoin d'une approche plus individualisée. En plus de gérer les tâches répétitives de l'enseignement, le système proposé doit tenir compte des différences individuelles des apprenants; c'est pourquoi il devra fournir une évaluation constante des apprentissages auquel l'enseignant pourra référer en tout temps afin de mieux orienter l'apprenant.

Nous examinerons dans un premier temps l'acte d'enseigner sous le regard d'un modèle de gestion. Cette démarche se fera en partant d'un modèle minimal et en l'améliorant successivement par l'ajout de composantes puisées des différents modèles du domaine de l'éducation. De là seront identifiés les attributs nécessaires à tout système d'enseignement.

Le système désiré étant également un système informatique, nous verrons comment l'ordinateur s'intègre pour aider l'apprenant à progresser en situation d'apprentissage. Nous aurons à étudier différents modèles tutoriels proposés en informatique pour en déduire une structure minimale d'un système d'enseignement assisté par ordinateur (EAO). De façon générale, un système EAO sera d'autant plus efficace que son degré d'adaptabilité à l'individu sera élevé [11]. II sera 
également montré que ce qui est souhaitable dans une situation d'apprentissage, l'est aussi pour tout autre situation de gestion.

Après avoir évalué les nécessités du modèle de gestion d'apprentissage et déterminé la structure minimale nécessaire à un système intelligemment assisté par ordinateur, il émerge un modèle théorique particulier. Notre choix se pose sur un modèle connu et largement utilisé: celui présenté par André Ouellet [37] qui semble répondre adéquatement aux attentes identifiées. Après cette justification nous aboutissons à la définition d'un modèle global opérationnel et d'un mode de progression. Nous démontrons ensuite la nécessité de gérer plusieurs bases de connaissances dont la nature des contenus et des fonctions diffèrent et pourront se déduire du modèle d'apprentissage analysé. La structure générale des bases de connaissances met en évidence le fait que chacune d'elles représente, dans une situation idéale, un système expert agissant sur chacun des contenus spécifiques des bases. De ce fait, chacune d'elles possèdent une structure indépendante et, idéalement, un système général prendra la forme d'un réseau de systèmes experts.

Etant donné que le système implique l'interrelation entre plusieurs bases de connaissances de natures différentes, le processus d'action du système nécessite une stratégie reposant nécessairement sur une structuration de règles à plusieurs niveaux afin d'éliminer toutes possibilités d'impasse. Les définitions et les justifications mathématiques des fonctions de transfert nécessaires entre les bases permettront de procéder de façon efficace à l'exécution du système.

Pour montrer la faisabilité d'un tel système, un exemple complet a été programmé: une situation d'apprentissage de l'algèbre élémentaire de Boole, et un deuxième exemple sera exposé sans être programmé: la gestion d'un bras manipulateur. Ces exemples illustrent les structures de connaissances définies plus tôt. L'utilisation du même système de gestion dans ces deux cas diamétralement opposés: l'un impliquant des ressources humaines et l'autre pas, démontre sa généralisation. 
Le programme qui gère la situation d'apprentissage de l'algèbre élémentaire de Boole porte le nom de PROLIN: PRO parce qu'il est écrit en langage Prolog et LIN pour Linda. Plusieurs simulations du programme ont été réalisées et elles ont permis d'obtenir des résultats qui nous semblent concluants. Cette analyse nous a conduit à des recommandations qui guideront l'utilisation ultérieure d'un tel système. 


\section{CHAPITRE I}

\section{L'ENSEIGNEMENT COMME GESTION DES RESSOURCES}




\section{CHAPITRE I}

\section{L'ENSEIGNEMENT COMME GESTION DES RESSOURCES}

\section{0: Introduction}

L'enseignement est à la fois un art et une science, de là sa complexité; aussi l'amélioration de l'un de ces volets ne peut qu'influencer positivement l'autre. Son caractère scientifique présente toutefois l'avantage de permettre la systématisation d'une situation d'apprentissage qui lui est propre et offre par le fait même la possibilité d'une gestion par ordinateur.

Le but de ce chapitre est d'expliquer comment il est possible de traiter une partie importante de l'aspect opérationnel de l'enseignement comme un système de gestion traditionnel. Cette démonstration est faite à l'aide de la présentation d'une succession de différents modèles du domaine de l'éducation. Nous construisons progressivement un modèle général par enrichissements successifs d'un modèle de base.

En parcourant les différents modèles d'apprentissage, le chapitre présente une réflexion sur les différents aspects de l'enseignement et de l'apprentissage. Cet examen montre que l'enseignement entraîne forcément une gestion de ressources humaines qui, dans ce cas, sont l'étudiant et l'enseignant. 


\section{1: Système de gestion de ressources}

Le dictionnaire Larousse définit le terme "ressource" comme "élément de la richesse ou de la puissance d'une nation". Sans individu capable de prendre des décisions importantes, sans capacité intellectuelle intéressante, une nation serait bien pauvre même si elle possédait en abondance des ressources minérales, hydrauliques, forestières, etc. Une nation sans personne pour gérer ses ressources naturelles ne peut se développer, encore moins s'enrichir; elle serait vouée à l'échec. Les ressources humaines sont donc sans conteste parmi les ressources les plus importantes.

Réaliser un apprentissage c'est, d'un certain point de vue, réaliser une transformation d'un apprenant à l'aide d'un processus. Considérons donc un système dont la matière première à l'entrée est l'apprenant avant" et, à la sortie, le produit fini représente l'"apprenant après". L'expression "apprenant avant" désigne l'état d'un apprenant avant l'événement d'apprentissage en cours et ne maîtrisant pas suffisamment les concepts véhiculés par l'événement d'apprentissage concerné. A la sortie, l'"apprenant après" désigne l'état de l'apprenant ayant réalisé les apprentissages conduisant à la maitrise des concepts enseignés au cours de l'événement. Ce processus peut se schématiser comme suit:

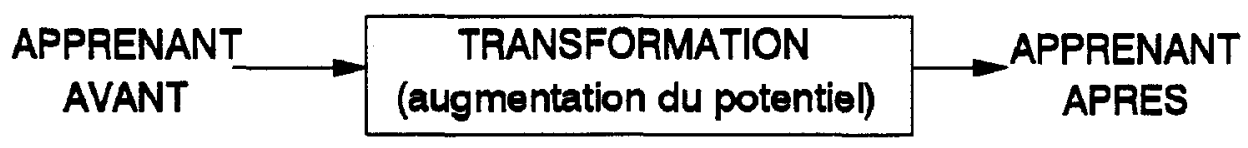

FIGURE 1.1 : Système général de transformation de l'apprenant

Ce système très simple est tiré directement, de par sa forme, de la boîte noire du système général de Le Moigne ([28] p.66). Remarquons que la transformation dans la situation qui nous intéresse représente une augmentation du potentiel intellectuel de l'apprenant par rapport aux concepts à maîtriser. 
"Explosons" maintenant les différents éléments de ce schéma et voyons comment les modèles d'apprentissage présentés par différents auteurs en pédagogie peuvent s'intégrer dans ce premier modèle. L'étude des approches dans le domaine de l'éducation nous permet de mieux cerner les volets importants à considérer et nous aidera ainsi à définir et à réaliser un modèle général de gestion.

\section{2: Première définition du modèle}

Bloom [04] nous propose un schéma (Figure 1.2) semblable à celui que nous avons proposé précédemment. Les entrées ou l'état de l'apprenant y sont définis comme étant les caractéristiques cognitives et affectives de l'élève. Les sorties sont les résultats obtenus après l'apprentissage et qui décrivent l'état de l'élève formé.

$\begin{array}{ll}\text { CARACTERISTIQUES } & \text { ENSEIGNEMENT } \\ \text { DEL'ELEVE } & \text { R'APPRENATS DE } \\ \text { LASSAGE }\end{array}$

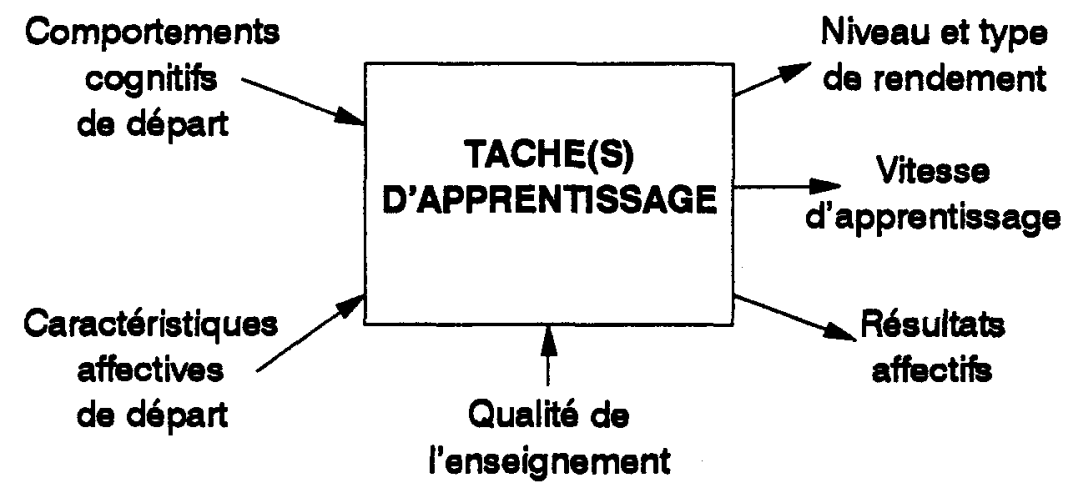

FIGURE 1.2 : Princlpales variables de la théorle de l'apprentlssage scolaire. 
Bloom insiste sur le rôle de l'évaluation. II est conscient du fait qu'il faut mesurer concrètement les résultats obtenus afin de vérifier s'il y a eu effectivement apprentissage. C'est d'ailleurs le problème majeur de tout formateur. L'intégration de la vision de Bloom à la figure 1.1 nous fournit un premier enrichissement du modèle de départ.

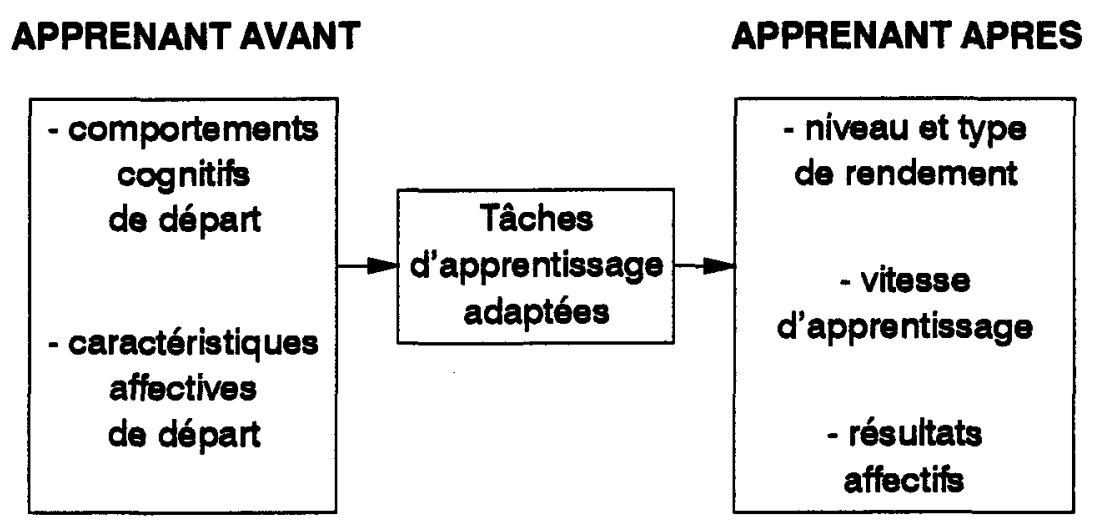

FIGURE 1.3 : Schéma général Inspiré de Bloom

On obtient un modèle où certains besoins importants sont mis en évidence comme:

-le besoin de bien connaître l'état initial des ressources, -le besoin de choisir adéquatement la fonction de transformation, et -le besoin de bien mesurer les effets obtenus.

Ces besoins représentent des qualités qui sont nécessaires à tout système de gestion de ressources (humaines ou non) mais ce ne sont pas les seules; d'autres aspects importants sont à considérer comme l'affirment plusieurs auteurs du monde de l'éducation. Leur examen permet d'identifier d'autres paramètres essentiels que doit considérer un système général de gestion. 


\section{3: Besoin de rétroaction}

Le volet scientifique de l'enseignement nous a permis de le voir comme un système de gestion, le deuxième volet entraîne pour sa part le fait que toute situation d'apprentissage est unique comme une oeuvre d'art. C'est donc l'art de l'enseignement qui amène à considérer chaque apprenant comme étant unique et ayant parfois besoin d'un rythme différent, d'un rappel, ou encore d'une toute autre approche. Toute situation d'enseignement repose toutefois sur les trois principes fondamentaux suivants (Provost [42]):

-les objectifs,

-la présentation du contenu,

-l'évaluation.

En incorporant ces trois principes dans notre modèle, nous obtenons une précision additionnelle qui fait ressortir l'importance de l'évaluation des connaissances acquises ou des effets obtenus de la transformation. L'évaluation permet de reconnaître si l'état désiré est atteint. Si cet objectif n'est pas atteint du premier coup, il faudra agir de nouveau en adaptant la stratégie, d'où on tire l'importance du concept de rétroaction ou de correction qui nous mène au schéma suivant.

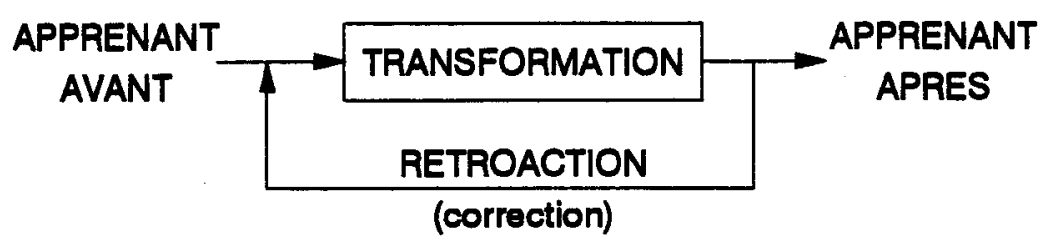

FIGURE 1.4 : Système général avec rétroaction 


\section{5: Besoin d'adaptabilité}

Les besoins de rétroaction et d'interaction, que nous venons de mettre en évidence, sont aussi prévus par Gagné [17] dans son modèle. II indique que les phénomènes d'apprentissage sont susceptibles d'être expliqués par des processus qui transforment, d'une façon analogue à celle de l'ordinateur, les "entrées d'information" en "sorties de résultats".

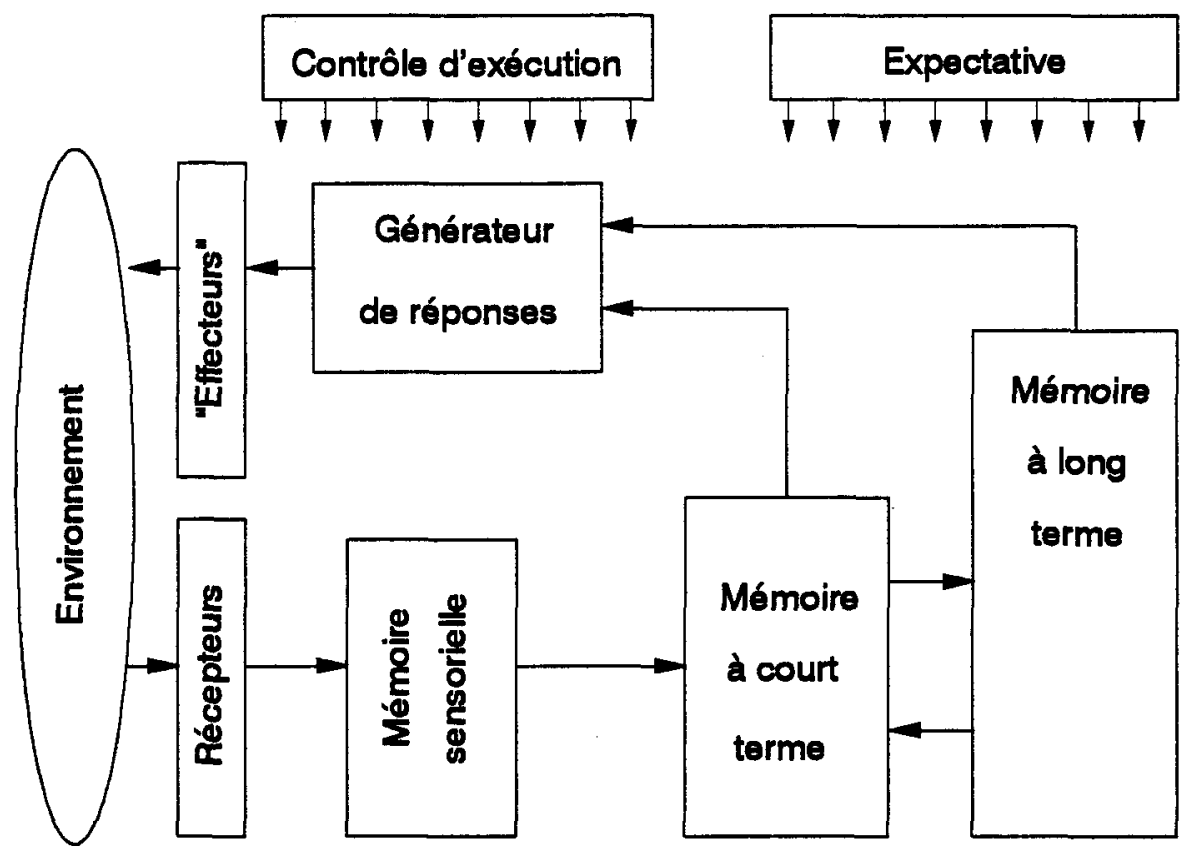

FIGUAE 1.5 : Le modèle de base de l'apprentissage et de la mémorisation qui sous-tend les théorles modemes du traltement de I'intormation. (Gagné [17] p.15)

Cette vision de Gagné enrichi le modèle en s'attaquant à la description des tâches d'apprentissage proprement dites; on y retrouve en effet le processus interne de l'apprentissage. II met cependant en évidence un nouvel élément: l'environnement qui est un des facteurs importants influençant la nature de la transformation de l'apprenant. 
L'environnement de l'apprenant, c'est d'abord la société dans lequel il vit. Ce sont les valeurs et les besoins de cette société qui entraîneront les besoins d'apprentissage et les méthodes d'enseignement à utiliser (Brien[05]). L'apprentissage se fait donc par et pour la société et l'enseignement représente une réponse aux besoins de cette dernière qui devient alors génératrice de situations d'apprentissage. II importe de bien définir ses états, ses objectifs et de les considérer comme des entrées du système.

Nous obtenons ainsi un système qui a pour entrées l'état de la société et les caractéristiques de l'élève (autant académiques, que psychologiques et motrices) et pour sorties les modifications de ces mêmes caractéristiques qu'ont engendré l'acte d'apprentissage. Toutes les entrées sont incluses dans l'environnement de l'apprentissage. Le schéma qui suit représente une réorganisation du modèle de Gagné qui incorpore le processus d'apprentissage à l'intérieur de cet environnement.

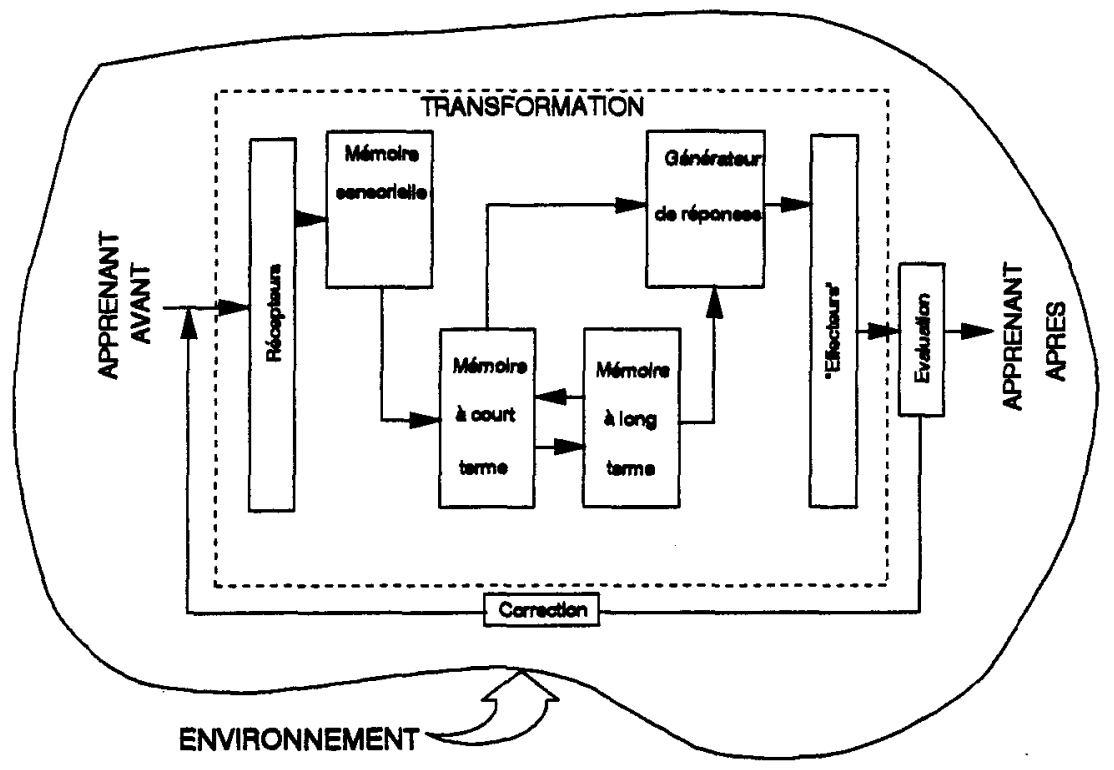

FIGURE 1.6 : Incorporation du modèle de Gagné dans le modèle général.

On peut réunir les théories de Gagné et Brien en disant qu'en plus d'être modifié par le processus de transformation, l'apprenant se modifie à l'intérieur d'un 
environnement (ou d'une société); le système doit être dynamique. En particulier, une des raisons qui fait de l'enseignement une transformation quasi-imprévisible est que chaque individu possède un profil d'apprentissage et un rythme qui lui est propre. Chaque être humain a, de par ses prédispositions psychologiques, des aptitudes différentes face aux diverses méthodes utilisées. Le processus doit donc tenir compte du contexte dans lequel il s'applique, contexte qui ne cesse de changer dans le temps.

De même, la motivation à apprendre est intrinsèque à tout apprenant l'amenant à tenter de donner un sens au monde qui l'entoure (Smith [50]); chacun aura sa propre façon d'y parvenir d'où l'importance de l'adaptabilité du point de vue stratégique. Un bon système de gestion de ressources (humaines ou non) devra être adaptatif de façon à pouvoir s'appliquer aux particularités qui se présenteront. Cette adaptabilité permettra de plus au système d'utiliser les différences individuelles de chaque apprenant dans ses choix de stratégies à la condition que le système possède un moyen efficace d'évaluer la situation.

\section{6: Besoin de plusieurs niveaux d'évaluation}

Le système adaptatif doit donc être en mesure, non seulement de s'adapter à tout individu, mais également à tout changement survenant en cours de transformation, ce qui conduit à la nécessité d'une évaluation à plusieurs niveaux. Une évaluation sommative est généralement employée dans l'enseignement traditionnel: elle considère la somme des résultats obtenus pour chacun des objectifs intermédiaires. Comme résultat global de l'apprentissage, nous visons une évaluation qui, en plus d'être sommative, sera contextuelle, normative et formative: une évaluation est contextuelle lorsqu'elle dépend des circonstances, normative si l'on peut en dégager des règles ou des préceptes et formative lorsqu'elle aide l'apprenant dans son apprentissage. L'utilisation de cette évaluation multiple rendra 
le système "intelligent", c'est-à-dire ayant un comportement qui ressemble à celui d'un humain.

\section{7: Conclusion}

Dans ce chapitre, nous nous sommes servi de l'acte de formation comme exemple général d'utilisation et comme définition du système qui nous intéresse. Nous l'avons modélisé comme un système de transformation de ressources humaines de façon analogue à toute autre ressource. Cet exercice a fait ressortir certaines qualités ou pouvoirs additionnels qu'un tel système doit posséder.

On a ainsi mis en évidence:

- le besoin de bien connaître l'état initial de la ressource,

- le besoin de choisir adéquatement la ou les fonctions de transformation,

- le besoin de bien mesurer les effets obtenus de la transformation,

- le besoin de rétroaction qui est nécessaire à tout système gestionnaire d'une situation d'apprentissage et est souhaitable pour tout autre système de gestion,

- le besoin d'adaptabilité du modèle au milieu culturel ainsi qu'au contexte cognitif et psychologique de l'apprenant,

- le besoin d'une évaluation multiple visant à renseigner sur:

- les états de la ressource,

- les acquis de la ressources par rapport aux objectifs fixés,

- les changements contextuels de la ressource,

- les changements contextuels de l'environnement, et

- l'utilisation d'une stratégie appropriée.

Un système pouvant utiliser tous ces renseignements à la fois sera sans nul doute capable d'une gestion efficace des ressources concernées. 
Dans un système de gestion autre que l'enseignement, ces nécessités paraissent moins évidentes. Toutefois, pour toute situation où il y a interaction avec des humains, ces qualités sont nécessaires. Incluons dans ces situations celles d'apprentissage et les cas de gestion de projet par exemple où il y a du personnel humain à gérer. Ce serait de même pour un problème de gestion d'entreprise pour lequel certaines décisions sont susceptibles d'influencer le milieu des affaires ainsi que la population en général si on pense aux emplois générés ou perdus, à la qualité de la vie, à l'environnement, etc.

Dans le cas où aucun humain n'entre en jeu, par exemple dans le cas d'un robot manipulateur ou encore celui de la gestion d'un problème théorique, le même modèle pourrait servir. Cependant, étant donné l'absence de contraintes psychologiques et sociales, il sera simplifié comme il le sera démontré dans la suite du mémoire.

Le modèle présenté peut donc, en plus d'agir sur les ressources humaines impliquées dans une situation d'apprentissage, englober bon nombre de systèmes qui implique les ressources naturelles.

L'analogie entre les ressources humaines (dans le contexte de l'enseignement) et les ressources naturelles (dans un système de transformation) justifie une approche scientifique de résolution de problèmes. L'enseignement peut ainsi être considéré non seulement comme un art mais également comme une science. Le modèle présenté dans cet ouvrage se veut en accord avec différentes approches du monde de l'éducation et pourra servir de base à tout système visant la résolution de problèmes en général. 
CHAPITRE II

\section{SYSTEME TUTEUR}




\section{CHAPITRE II}

\section{SYSTEME TUTEUR}

\section{0: Les différents systèmes tuteurs}

Il existe de plus en plus d'outils informatiques performants qui peuvent représenter le modèle éducationnel que nous avons identifié au chapitre précédent. Ces outils visant la transformation d'un apprenant, il est nécessaire d'avoir constamment une définition de l'état de l'usager, d'où le modèle étudiant.

\subsection{1: Le modèle étudiant}

Tous les systèmes tutoriels doivent obligatoirement inclure un modèle de l'étudiant contenant le profil des apprentissages de ce dernier. Cet ensemble de référence permettra de juger la progression de l'apprenant par rapport aux objectifs poursuivis.

Parmi les nombreux outils de gestion de situations d'apprentissage existants, certains sont plus élaborés que d'autres; on peut les classifier en trois catégories:

- les systèmes tutoriels conventionnels,

- les systèmes tutoriels intelligents, et

- les systèmes tutoriels intelligents adaptatifs. 
Cette classification est le résultat de l'intervention de différentes disciplines comme le montre la figure suivante.

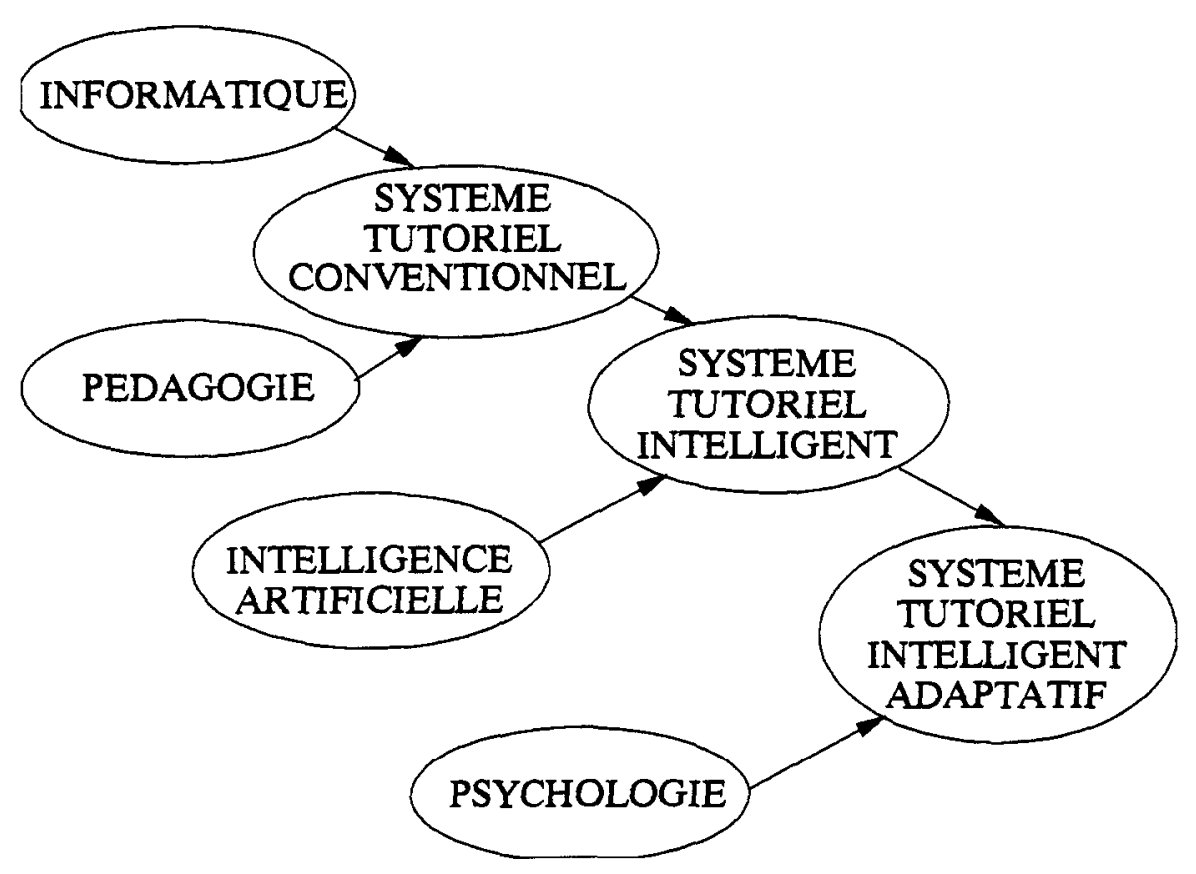

FIGURE 2.1: Princlpaux systèmes tutorlels

On appelle "système tutoriel" un outil permettant de gérer une situation d'apprentissage ou des logiciels ayant pour objectif d'aider une personne à comprendre et à approfondir un sujet donné.

\subsection{2: Système tutoriel conventionnel}

Le plus simple des tutoriels utilise l'informatique et la pédagogie; les programmes skinnériens (Depover [11]) où la progression est lente et presque toujours linéaire, sont de ce type. L'efficacité d'un tel système, qui avance à pas uniformes sans égard à l'état actuel de la situation est restreinte. Pour un robot, ceci pourrait signifier de faire des pas ayant tous une longueur de un centimètre, ce qui représente une progression beaucoup trop lente si le but visé se trouve à un mètre de distance ou beaucoup trop rapide si le but se situe à trois millimètres. Le 
mode de progression du système a donc une importance fondamentale et l'intelligence artificielle permettra au système de conduire "intelligemment" cette progression.

\subsection{3: Système tutoriel intelligent}

Le système tutoriel intelligent diffère du tuteur conventionnel par le fait qu'il utilise, non plus une vision déterministe (en comparant avec des valeurs exactes), mais qu'il emprunte un comportement plus humain en acceptant une certaine marge de manoeuvre. Cette marge de manoeuvre nous aide à obtenir une structure plus souple qui, dans un contexte d'enseignement, permettra d'évaluer l'usager selon les objectifs à atteindre, le programme envisagé, les activités proposées, etc. Les techniques de l'intelligence artificielle permettront de ralentir ou d'accélérer la progression de l'apprentissage selon les besoins circonstanciels et selon le cumul des résultats obtenus lors des apprentissages antérieurs.

Nous obtenons ainsi un moyen de tenir compte de l'individualité académique de l'apprenant. Cela représente un progrès appréciable par rapport au système tutoriel conventionnel. Chaque apprenant possédant son propre profil d'apprentissage et se distinguant des autres par des composantes psychologiques, l'individualisation devient nécessaire.

\subsection{4: Système tutoriel adaptatif}

Le respect de l'individualisation et des composantes psychologiques de l'apprenant nécessite l'adaptativité du modèle. L'ajout de certaines composantes psychologiques de l'apprenant nous conduit à la notion de système tutoriel intelligent adaptatif. Le système visé dans ce travail fait partie de cette catégorie.

Après avoir bien cerné nos besoins par rapport aux différents modèles existants, nous définirons une nouvelle structure minimale nécessaire à la gestion d'un système tutoriel intelligent adaptatif en incorporant deux rôles possibles de 
l'ordinateur dans l'enseignement. Cet exercice permettra de mettre en évidence les besoins et rôles de chacune des composantes du système visé.

\section{1: Le modèle étudiant}

Une modélisation de l'apprenant (le modèle étudiant) permet au système de faire des choix éclairés face l'individualité de ce dernier. II y a plusieurs façons de définir les caractéristiques de l'élève mais, en priorité, c'est le niveau d'acquisition des connaissances à maîtriser qui est à considérer. En définissant les sujets qui lui sont acquis, nous situons l'apprenant à l'intérieur d'un ensemble de connaissances à atteindre. Ces dernières, gérées à l'intérieur d'un modèle étudiant, permettent au système de faire un choix judicieux d'activités d'apprentissage et de fournir à l'apprenant l'exercice le plus performant selon sa situation. La question qui se pose maintenant est: "Comment pouvons-nous modéliser le savoir de l'appreriant?"

L'apprenant ne pourra être modélisé que dans un espace limité à un ensemble de référence restreint spécifié par l'expert comme, par exemple, le programme à apprendre et ses prérequis immédiats. En situant l'apprenant à l'intérieur d'un tel référentiel, on considère sa connaissance comme un sousensemble de celle d'un expert (Figure 2.2). 


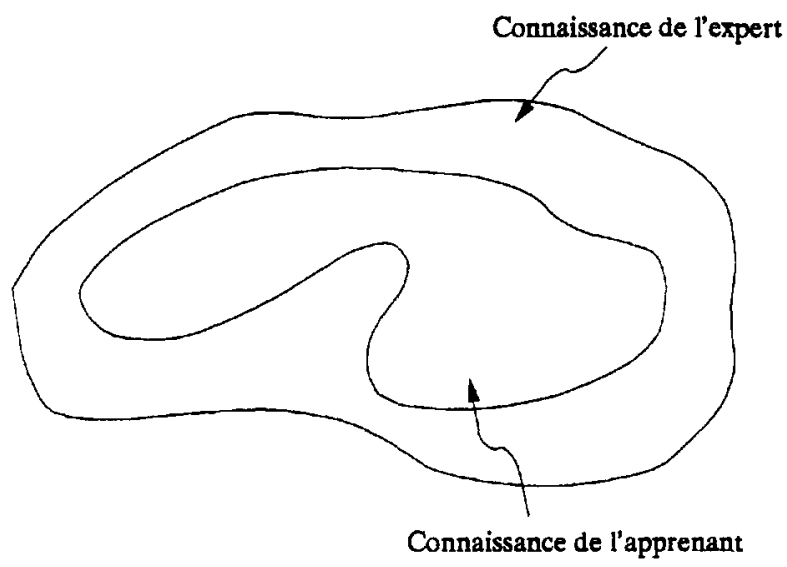

FIGURE 2.2 : Connalssance comme sous-ensemble de celle d'un expert (Kass

[26]

Le fait de considérer la connaissance de l'apprenant de cette façon, simplifie le problème puisqu'il suffira d'évaluer à chaque pas l'amélioration de la connaissance de ce dernier. On considère que l'élève a réussi les objectifs lorsque le recouvrement des connaissances de l'expert est satisfaisant (Figure 2.3).

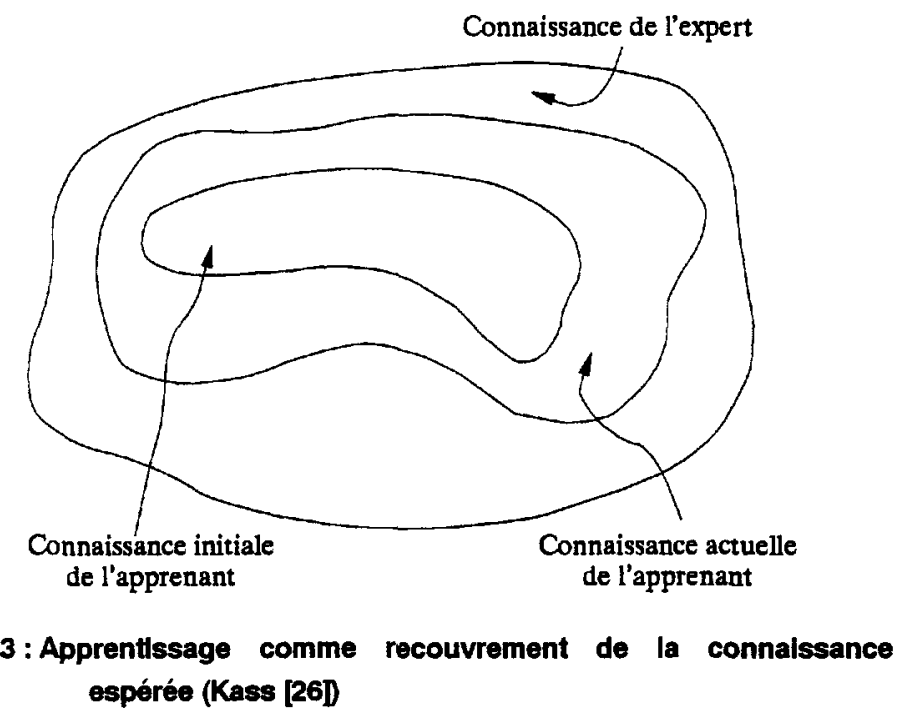

Cette approche possède toutefois un inconvénient car il est possible qu'en comparant les connaissances de l'apprenant et de l'expert, on découvre des 
distorsions. Ces dernières représentent des connaissances possédées par l'apprenant mais ignorées de l'expert (Figure 2.4).

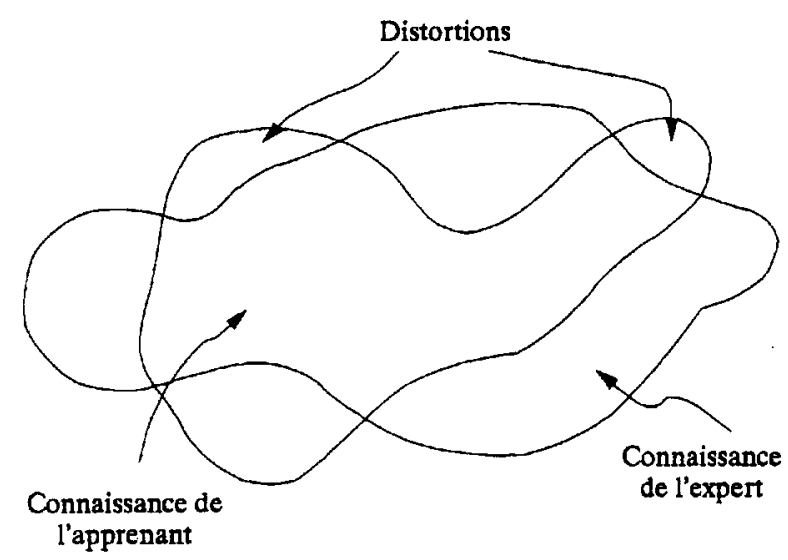

FIGURE 2.4: Distorsions (Kass [26]

Il ne faut pas négliger ces distorsions. Le fait qu'elles soient inconnues de l'expert n'implique pas nécessairement qu'elles soient fausses. Lorsqu'un élève donne à l'enseignant une réponse que ce dernier ne connaît pas, il ne peut la valider; il doit donc vérifier à une source extérieure, en faisant appel possiblement à un autre expert.

Le système tutoriel que nous développons devra agir de la même façon. En cas d'impasse il questionnera l'enseignant pour qu'il complète son domaine de connaissances afin d'inclure complètement celui de l'apprenant. Cette opportunité rend le système ouvert à recevoir en tout temps un complément de connaissances de manière à ce qu'il demeure en mesure de répondre aux besoins de l'apprenant. 


\section{2: Système tutoriel conventionnel}

En pédagogie Smith et Blandford [51] nous proposent une structure de simulation d'enseignement assistée par ordinateur qui s'apparente à la définition que nous avons donné au système tutoriel conventionnel.

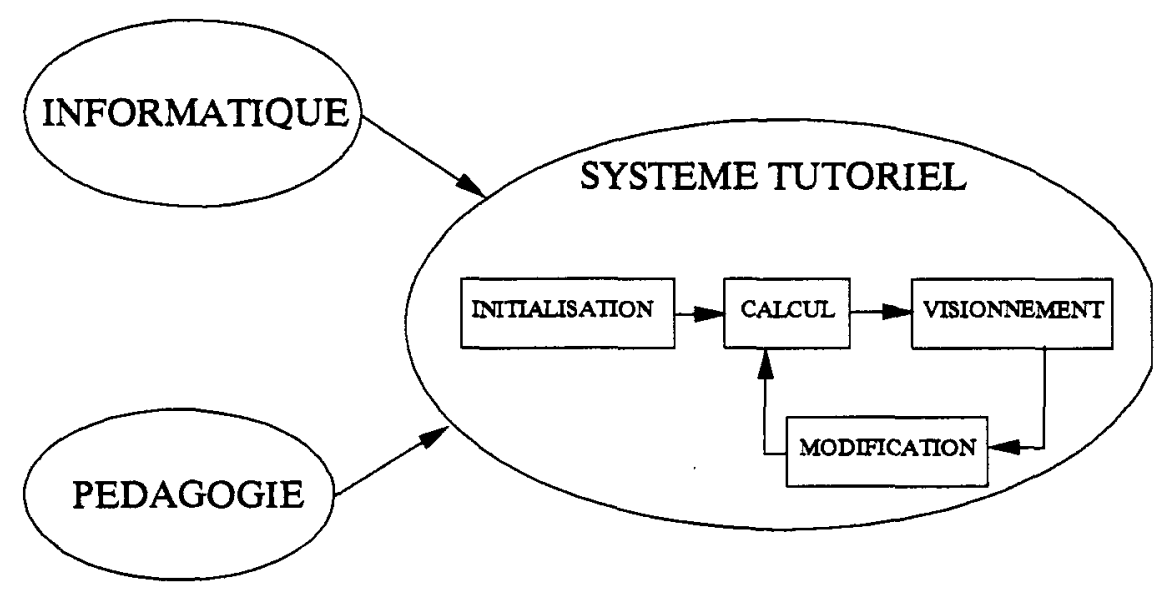

FIGURE 2.5 : Structure de simulation d'enselgnement (Insplré de Smlth et Blandford [51] p.53)

Faisant appel à des notions de l'informatique et de la pédagogie, cette structure très simple met en évidence le déroulement répétitif de la situation d'apprentissage. Elle comprend quatre phases ayant chacune un rôle important:

- l'initialisation où sont déterminées les valeurs initiales des paramètres requis pour réaliser la prise de décisions;

- la phase calculatoire où sont choisies les activités nécessaires à l'apprentissage. C'est la partie tuteur, le coeur du système tutoriel;

- le visionnement où est montré à l'usager le résultat de la décision se concrétisant par une activité à faire. C'est à ce niveau que l'apprenant réagit avec le système en répondant à une question; 
- la modification où sont ajustées les valeurs des paramètres impliqués en fonction de la réponse obtenue au visionnement. Cette modification du modèle de l'étudiant est suivie du retour à la phase calculatoire et permet de poursuivre le processus d'apprentissage de façon cyclique jusqu'à l'atteinte des objectifs visés.

Ce point de vue de Smith et Blandford, à la fois simple et circonscrit, présente la boucle minimale d'intervention d'un système tuteur. Cette vision met l'emphase sur la nécessité de rétroaction intrinsèque du processus d'apprentissage, concept fondamental à la base de tout tuteur d'apprentissage. La partie tutorielle se situe en fait au niveau de la phase calculatoire, c'est là qu'interviennent à la fois l'étudiant (au niveau du visionnement via l'interface de communication), le programme à apprendre (faisant partie de l'initialisation) et le modèle de l'étudiant (rendant possible la prise de décision relative à la modification à effectuer).

\section{2: Système tutoriel intelligent}

Un système tutoriel faisant usage de techniques de l'intelligence artificielle est qualifié de système tutoriel intelligent (Intelligent Tutorial System ou ITS) [10]. L'intelligence artificielle permet de réaliser une évaluation à niveaux multiples, une qualité importante recherchée pour les systèmes d'apprentissage (chapitre 1). On peut ainsi évaluer de façon nuancée les concepts acquis, les stratégies utilisées par l'apprenant, la vitesse de résolution des problèmes, etc. De plus, l'évaluation des stratégies utilisées par le système permet d'ajuster le déroulement de l'intervention pédagogique. 


\subsection{1: Un exemple de système tutoriel intelligent: GUIDON}

Le système GUIDON (Clancey [10] 1987), un système d'aide à l'apprentissage en médecine, se classe parmi les systèmes tutoriels intelligents.

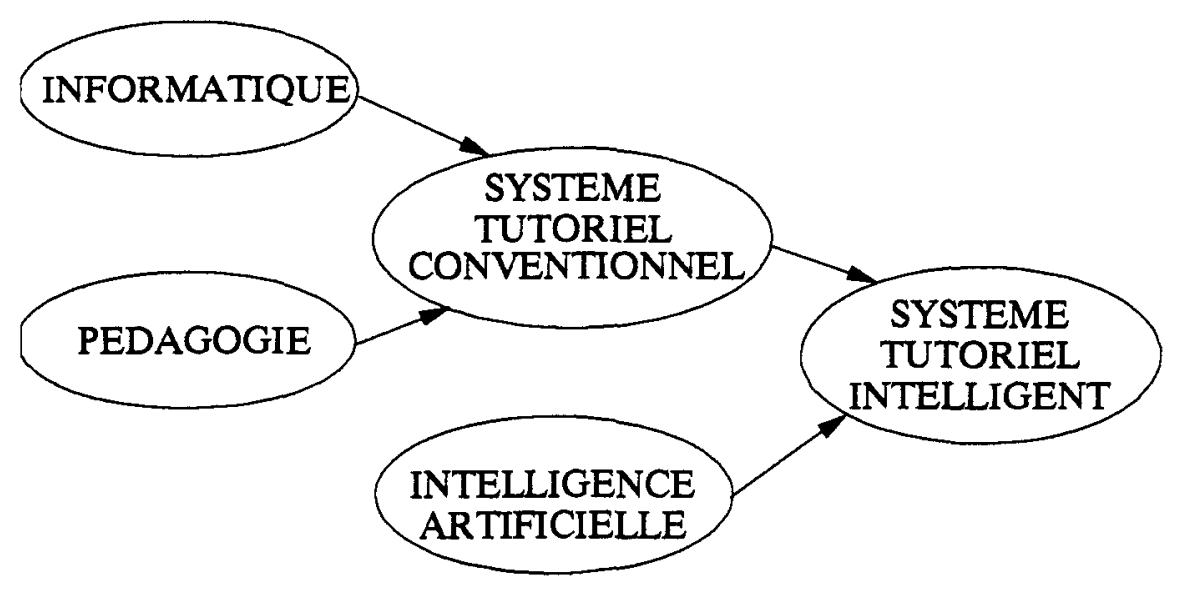

FIGURE 2.6 : Système tutoriel Intelligent (Fugère, Goleyn Tremblay [16]

Le système GUIDON s'appuie sur l'apprentissage par étude de cas et est structuré comme suit. 


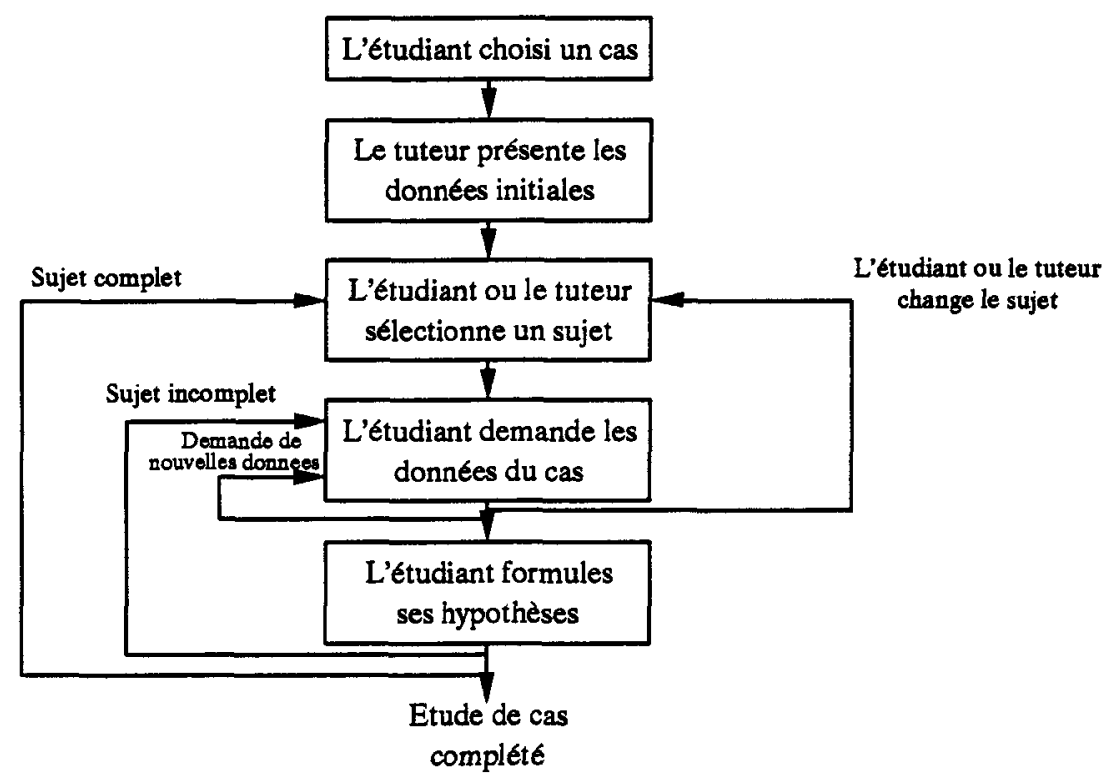

FIGURE 2.7 : Structure générale de GUIDON (Clancey [10] p.5)

Un modèle étudiant par recouvrement est utilisé: la connaissance du médecin apprenant est modélisée en termes de sous-ensembles et de variations simples de la connaissance de l'expert. Cet expert, appelé MYCIN, est un système à bases de connaissances très performant utilisé pour le dépistage des maladies infectieuses. Le rôle de GUIDON est d'utiliser l'expertise de MYCIN afin de répondre aux questions de l'étudiant médecin. Ainsi, GUIDON soumettra au système expert MYCIN les renseignements que le médecin lui propose. GUIDON utilisera ensuite la réponse de MYCIN pour fournir au médecin les réponses à ses questions et lui expliquer pourquoi l'expert considère un diagnostic plutôt qu'un autre. De plus, MYCIN résout les différents problèmes proposés au médecin apprenant rendant possible l'évaluation adéquate de ses réponses. La structure souple de GUIDON permet à l'étudiant de demander à sa guise des données supplémentaires ou encore d'explorer l'arbre de décision dont se sert l'expert MYCIN.

Lors de la comparaison des conclusions de l'apprenant avec celles de MYCIN, il est possible que l'on se retrouve en zone de distorsion (Kass[26]). 
Clancey [10] surmonte ce problème en utilisant une interface (TEIRESIAS) de transfert d'expertises entre l'expert humain et le système.

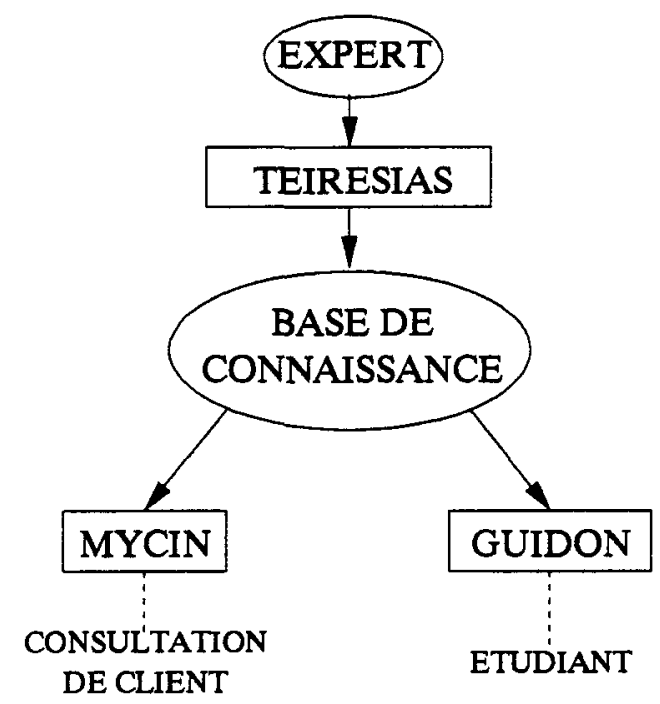

FIGURE 2.8 : Transfert d'expertise: apprentissage, consell et enselgnement (Clancey [10] p.9)

TEIRESIAS consulte l'expert humain afin de nourrir une base de connaissances qui alimente à son tour MYCIN et GUIDON. TEIRESIAS construit et maintient la base de connaissances de MYCIN (et de GUIDON par le fait même) en interaction constante avec les experts humains permettant au domaine de connaissances de MYCIN de toujours inclure celui de l'apprenant.

Ce qu'on demande à un système tutoriel d'apprentissage, c'est de partir d'un programme et d'un modèle étudiant pour alimenter un tuteur qui offrira à l'apprenant des activités d'apprentissage via une interface. Le système tutoriel agira donc dans le sens inverse de TEIRESIAS mais, comme l'apprenant réagira également aux activités présentées, il y aura un mouvement dans les deux sens. En effet, on désire développer un modèle étudiant qui fournira des informations au tuteur et qui sera modifié par ce dernier, ce qui nous conduit au schéma suivant. 


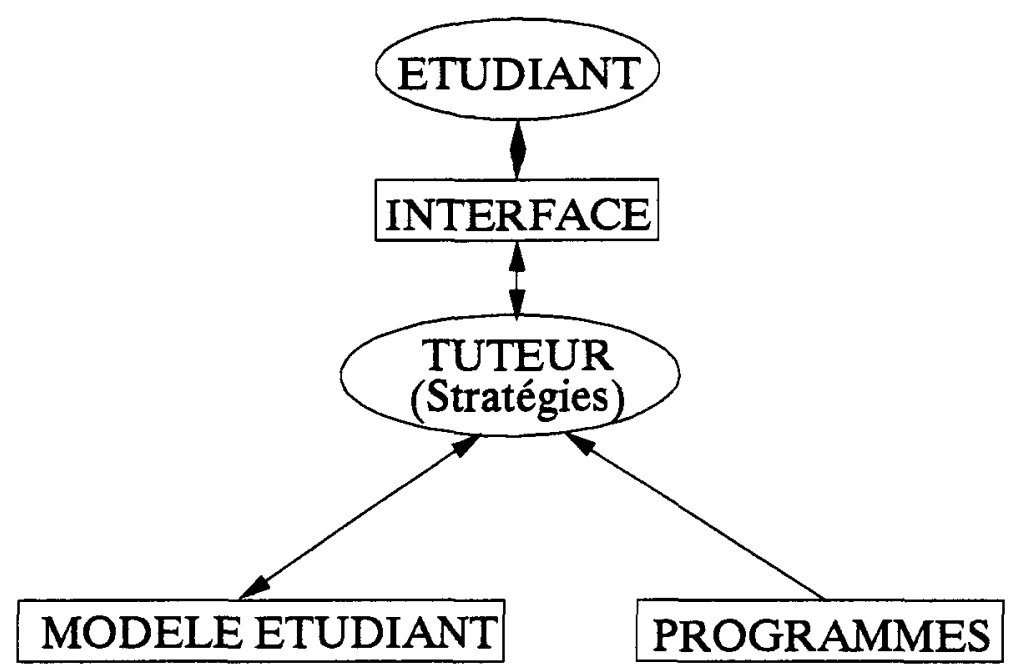

FIGURE 2.9 : Tuteur d'enselgnement (Fugère, Geleyn, Tremblay [16]

Remarquons que ce système sera d'autant plus efficace qu'il pourra corriger en temps réel le modèle étudiant, d'où on tire un besoin d'adaptabilité.

\section{4: Système tutoriel intelligent et adaptatif}

L'utilisation d'un système tutoriel intelligent, comprenant un modèle étudiant, nous apporte, comme on l'a vu, une certaine souplesse et adaptabilité. Toutefois, dans le domaine de l'enseignement intelligemment assisté par ordinateur (EIAO), l'individualité de l'apprenant est trop souvent restreinte au rythme d'apprentissage de l'apprenant alors qu'on pourrait en espérer beaucoup plus.

Chaque individu possède un style d'apprentissage qui lui est propre (Despins [12]), ce qui demande la mise en place de stratégies pédagogiques différentes. Le style de la stratégie utilisée devrait également dépendre des moyens de communication privilégiés de l'apprenant puisque l'apprentissage doit être 
satisfaisant pour ce dernier (Lamontagne [27]). De plus, pour aider à sa perception, il importe de tenir compte des modes de perception et de conceptualisation de l'apprenant si on recherche un traitement dirigé-par-concepts (Lindsay et Norman [31]). Ces modes de fonctionnement étant propres et particuliers à chaque personne, le tuteur d'apprentissage intelligent adaptatif devra tenir compte de ces particularités qui relèvent de la psychologie.

En ajoutant au système tutoriel intelligent, certains éléments de la psychologie, on obtient un système tutoriel intelligent adaptatif (Adaptative ICAI ou Adaptative Intelligent Computer Aid Instruction). L'adaptabilité du système peut également concerner certains paramètres physiques: l'apprenant peut par exemple être sourd, on peut aussi se heurter à des contraintes de temps, d'espace ou de liberté de mouvement. A chaque fois qu'on se trouve face à des contraintes individuelles, c'est-à-dire qui dépendent de l'usager, l'adaptabilité prend une importance particulière. Ce fait peut également s'appliquer à un contexte machinemachine comme la gestion d'un bras manipulateur.

Le système que nous visons est un système tutoriel intelligent adaptatif, nous rechercherons dans les prochaines lignes à en expliciter la structure. 


\section{5: Recherche d'une nouvelle structure minimale}

Lewis [30] distingue deux rôles associés à l'ordinateur dans un contexte d'enseignement:

- il peut aider l'apprenant en jouant un rôle de didacticien comme on le retrouve dans la plupart des tuteurs conventionnels existants,

- il peut aider l'enseignant en jouant un rôle de ressource d'investigation en parallèle. Il sert à générer de façon ponctuelle des exemples et problèmes qui pourront éventuellement être proposés à l'apprenant.

Le système que nous préconisons intègre simultanément ces deux rôles. Cette intégration possède l'avantage de donner au système informatique un haut degré d'autonomie lui permettant de supporter de façon constante l'enseignant dans la plupart des situations.

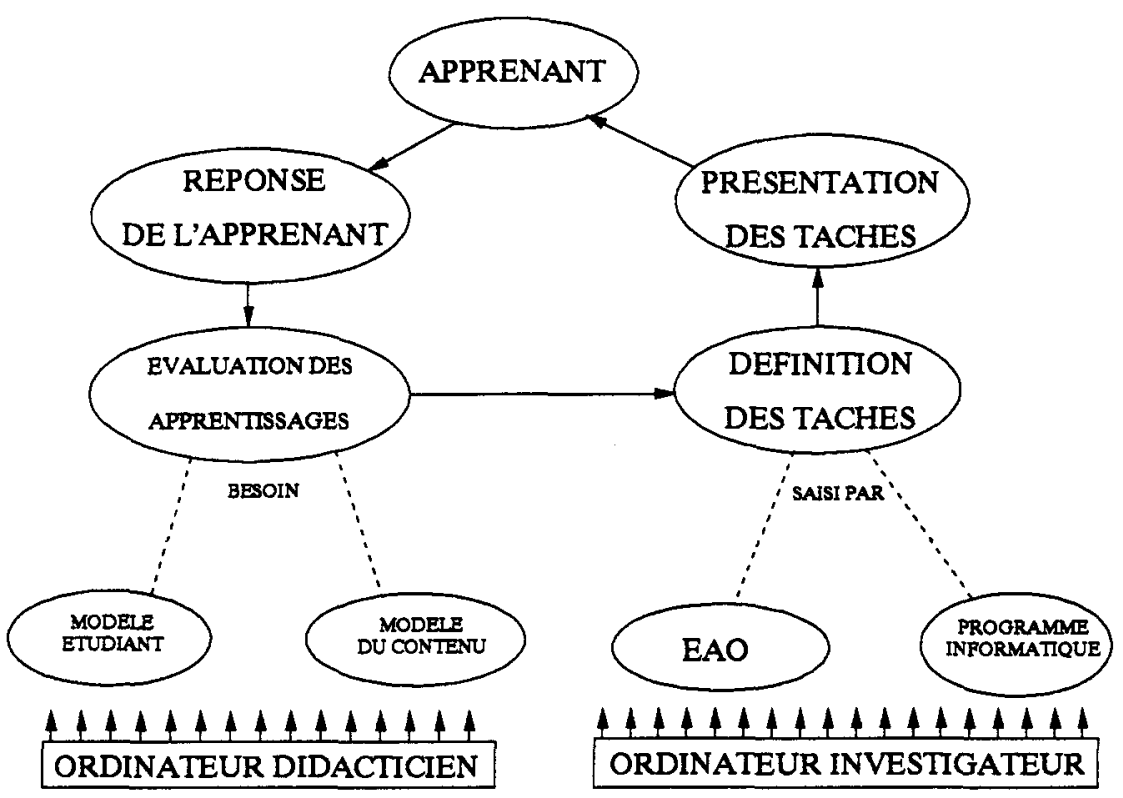

FIGURE 2.10: Système tuteur à deux nlveaux pour la gestlon d'une sltuation d'apprentlssage. (Insplré de Lewls [30]) 


\subsection{1: Application à la robotique}

L'intégration des deux niveaux de contrôle trouve sa justification dans d'autres situations complètement différentes comme en robotique où l'apprenant est remplacé par un robot. La présentation des tâches d'apprentissage se résume alors à donner des ordres au robot et la réponse de l'apprenant devient le mouvement effectif résultant de l'ordre donné( Figure 2.11).

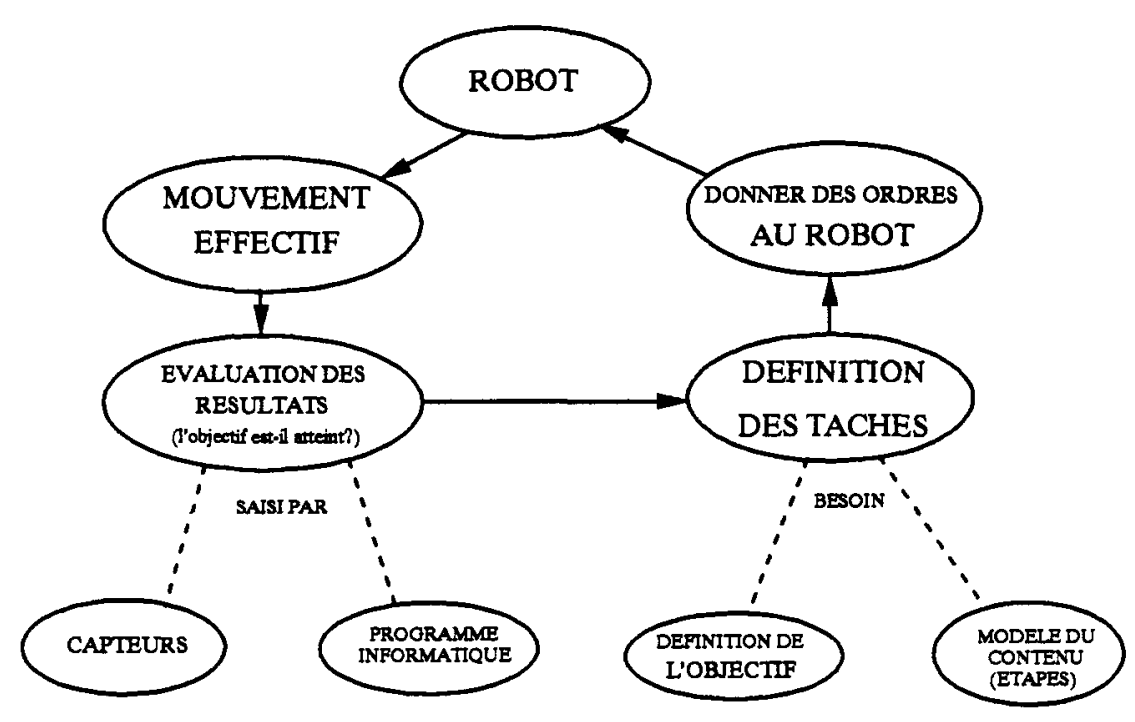

FIGURE 2.11: Système tuteur à deux niveaux pour la gestion d'un
robot.(nspliré de Lewls [30]

Nous venons de mettre en évidence un système dont l'un des niveaux trouve intelligemment les tâches à soumettre pendant que l'autre s'occupe de la didactique de l'apprentissage conditionnée par l'évaluation des résultats obtenus. Les deux rôles possibles de l'ordinateur entraînent une définition plus spécifique de la structure visée (Figure 2.9) davantage en accord avec celle de Smith et Blandford (Figure 2.5). 


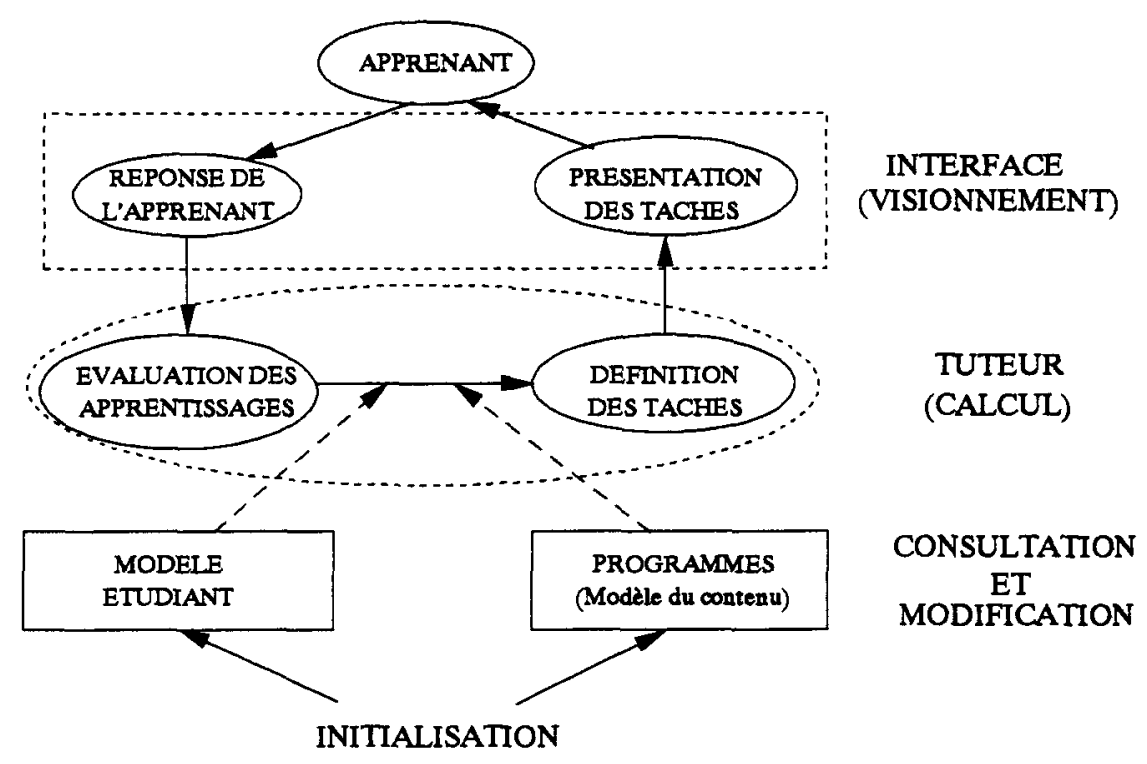

FIGUAE 2.12: Tuteur gestionnalre d'une situation d'apprentissage

Le tuteur utilise le programme et le modèle étudiant afin de définir des stratégies d'apprentissage (phase calculatoire). Ensuite il soumet ces stratégies à l'interface qui les présente à l'étudiant sous forme d'exercices, de questions, d'explications, etc. (phase de visionnement). Puis l'apprenant répond à la demande du tuteur. Cette réponse est interprétée par le tuteur qui modifie au besoin le modèle étudiant contenant les résultats de l'élève. Le tuteur consulte également le programme et détermine les prochains sujets à soumettre. Ce cycle recommence jusqu'à l'obtention du rendement désiré. 


\section{5: Conclusion}

A l'aide de structures puisées du champ d'expertise de l'enseignement assisté par ordinateur et tenant compte des besoins établis au chapitre 1, nous avons défini un modèle conceptuel minimal et général. La démonstration de la nécessité de l'ajout d'un modèle étudiant a permis de le qualifier de système tutoriel. L'ajout de notions de l'intelligence artificielle a conduit au concept de système tutoriel intelligent. Le qualificatif "adaptatif" fut justifié par la considération de composantes psychologiques de l'apprenant. L'identification et l'intégration des deux rôles possibles de l'ordinateur dans l'enseignement ont entrainé une définition plus riche encore de la structure visée.

Cette individualisation et personnalisation de l'intervention demande par surcroît une interactivité de haut niveau entre le tuteur et l'apprenant, contribuant à rendre le système encore plus complexe. Nous tenterons dans les prochains chapitres de proposer un modèle général qui pourrait éventuellement tenir compte de tous les paramètres identifiés précédemment. 
CHAPITRE III

\section{LE MODELE UTILISE}




\section{CHAPITRE III}

\section{LE MODELE UTILISE}

\section{0: Introduction}

Les chapitres précédents ont été consacrés à la problématique rencontrée en situation d'apprentissage. Dans ces derniers les principales caractéristiques souhaitables à tout système de gestion en de telles situations ont été identifiées. Le présent chapitre présente le modèle sur lequel se fonde le système informatique de gestion que nous proposons. Ce modèle tient compte de la plupart des caractéristiques identifiées précédemment. Nous terminerons ce chapitre par la présentation de l'algorithme développé pour opérer cette gestion.

Parmi les nombreux modèles existants (Bloom, Provost, Gagné, Lewis, Le Moigne, Ouellet, etc.), nous retenons celui présenté par Ouellet [37],lequel origine de la théorie du système général de Le Moigne [28]. Ce modèle possède les propriétés d'auto-évaluation et de rétro-action qui sont des propriétés essentielles pour ce type de système. 


\section{1: Le système général}

\subsection{1: Le modèle de Le Moigne}

Le modèle présenté par Ouellet s'inspire de la théorie de Le Moigne [28] qui présente la définition d'un système général. Cette définition nous apprend beaucoup sur la structure qu'il faudra considérer.

Un système général au sens de Le Moigne en est un pour lequel la structure interne évolue au fil du temps, sans perdre son identité unique (Le Moigne [28]). Plus succinctement, un tel système:

- représente quelque chose (n'importe quoi, identifiable)

- fait quelque chose (activité = fonction),

- possède une structure,

- évolue dans le temps,

- fait partie de quelque chose (environnement),

- vise quelque chose (finalité).

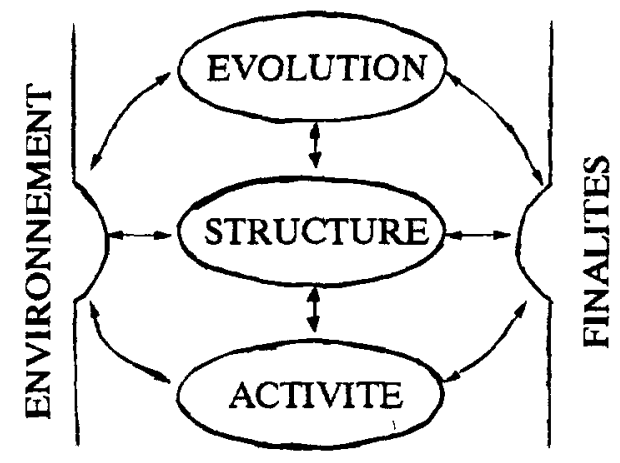


Le système général proposé par Le Moigne comble l'ensemble des besoins mis en évidence au chapitre 1. Il englobe aisément la gestion d'un système tutoriel intelligent adaptatif (chapitre 2). Ce modèle général peut, de plus, satisfaire toute situation de résolution de problèmes.

\subsection{2: Le modèle de Ouellet}

Ouellet [37] adapte la vision de Le Moigne à la situation d'apprentissage vue comme une transformation. II propose le modèle suivant.

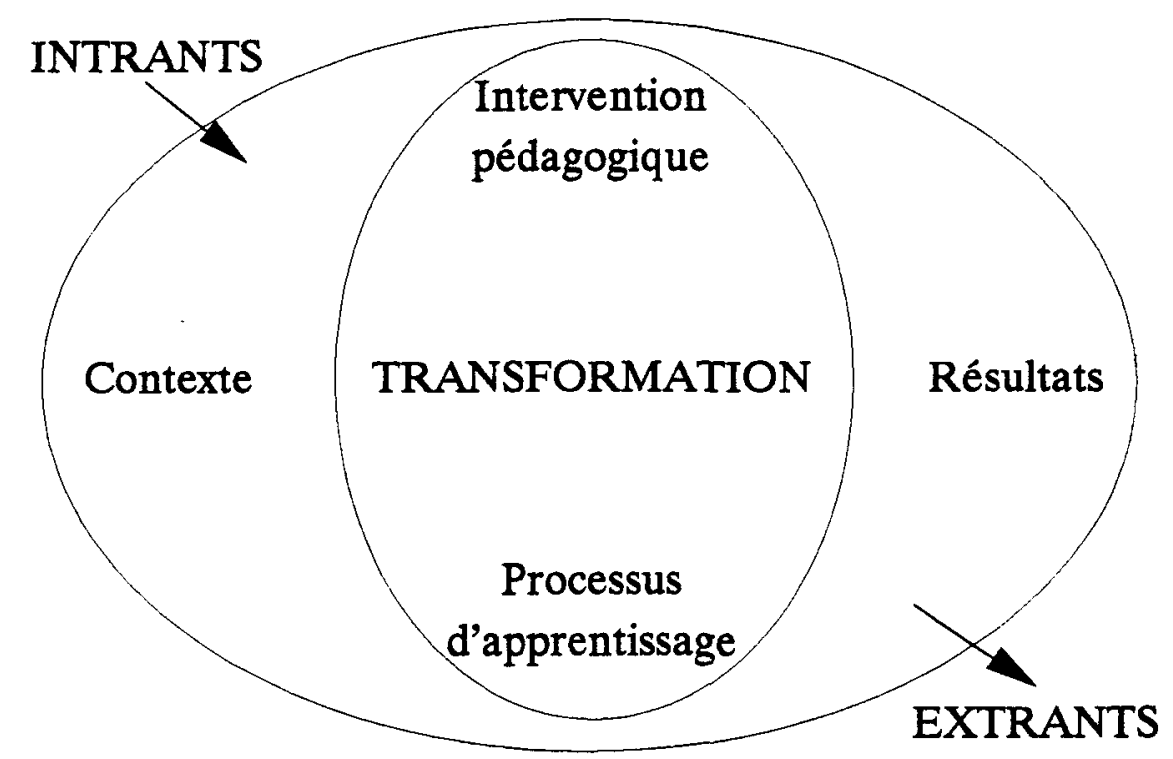

FIGURE 3.2 : Organisation des données de I'Intrant, de la transformation et de l'extrant (Ouellet [37] p.103)

Les intrants d'un tel système sont les éléments du contexte (situation environnementale, agents) et les extrants sont les résultats (habiletés, attitudes, connaissances, coûts). La transformation se situe à l'intérieur d'une boîte noire intermédiaire et représente le système d'apprentissage où l'enseignement s'effectue selon les stratégies appropriées. Cette transformation comprend 
l'intervention pédagogique (programme, objectifs, méthodes) et le processus d'apprentissage (activités). II s'agit essentiellement d'un système de transformation d'une ressource à partir d'un état initial ('lapprenant avant apprentissage) vers un état final (l'apprenant après apprentissage).

Ce modèle met en présence quatre ensembles de faits en constante interaction (figure 3.3):

- un contexte représentant l'environnement physique et social de l'apprentissage,

- une intervention pédagogique définissant l'action concrétisée par l'activité d'apprentissage,

- un processus d'apprentissage comprenant la réaction et représentant l'acquisition des concepts désirés,

- l'ensemble des divers résultats représentant l'évaluation des apprentissages et des techniques utilisées.
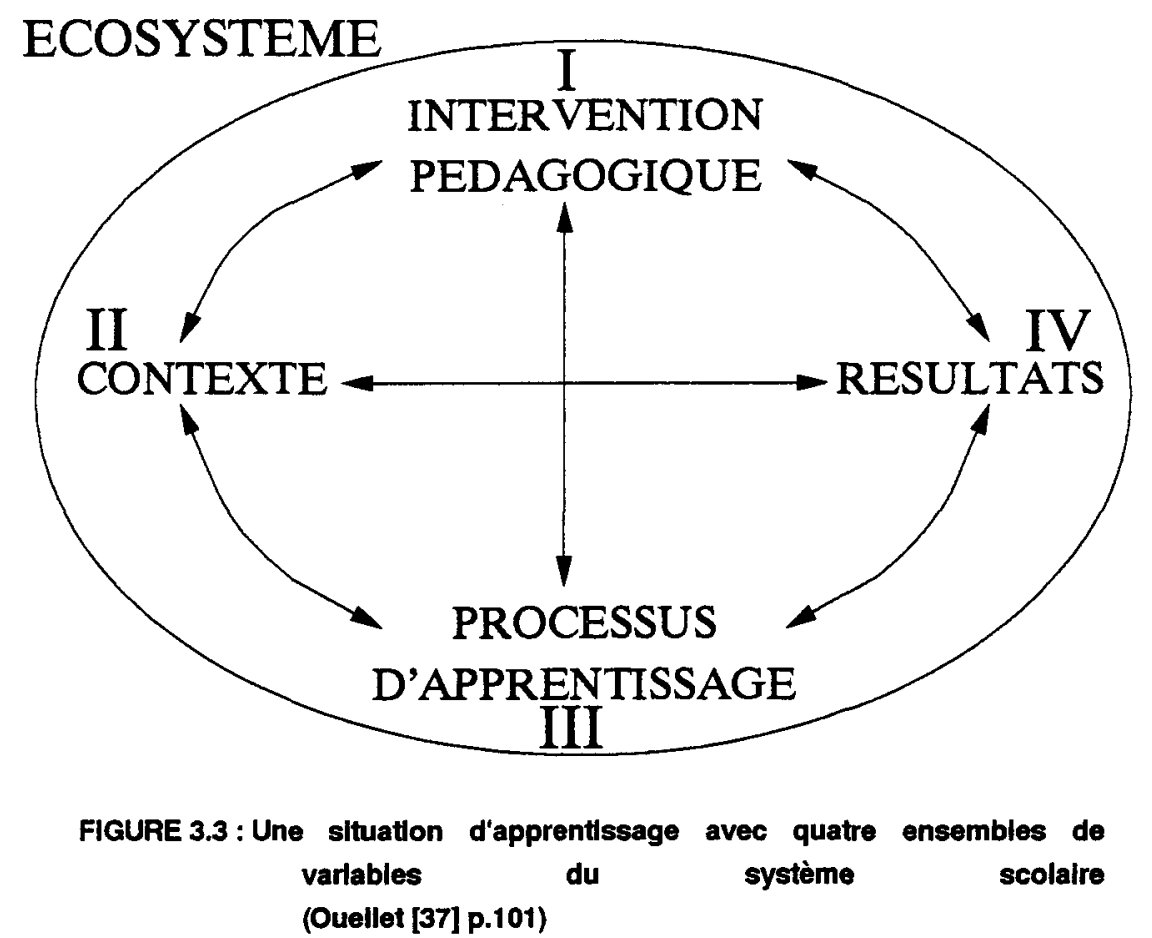
Les quatre ensembles de variables identifiés I, II, III et IV dans la figure 3.3 représentent essentiellement quatre types de connaissances de natures différentes mais interdépendantes. Ouellet insiste sur la nécessité d'équilibrer constamment l'ensemble des paramètres en situation réelle d'apprentissage.

Pour réaliser cet équilibre, il suggère les bases d'un dialogue entre les ensembles se traduisant par une suite ordonnée de quatre questions pour chacun des quatre ensembles s'adressant aux trois autres selon un ordre déterminé.

A titre d'exemple, considérons l'ordre de questionnement suivant entre les bases: I, II, III et IV.

Partant de l'intervention pédagogique, on se pose comme première question:

Question 1: (niveau 0 de questionnement): "Quel est l'objectif visé?".

Par la suite, il faut dans l'ordre:

- préciser le contexte ou se demander:

Question 2: "Qui (niveau 0 de questionnement) doit atteindre cet objectif (niveau 1 de questionnement)?",

- préciser le processus d'apprentissage ou s'interroger comme suit:

Question 3: "Parmi les moyens dont je dispose (niveau 0 de questionnement), lequel est le plus efficace compte tenu du contexte (niveau 1 de questionnement) pour atteindre l'objectif visé (niveau 2 de questionnement)?",

- préciser le résultat ou se poser la question:

Question 4: "Quel est le résultat espéré (niveau 0 de questionnement) compte tenu du processus utilisé (niveau 1 de questionnement), du contexte (niveau 2 de questionnement) et de l'objectif à atteindre (niveau 3 de questionnement)?".

L'exemple précédent représente un des vingt-quatre (24) ordres possibles (4!) générant autant de questions. Le réseau de communication est représenté par des flèches bidirectionnelles dans la figure 3.3. 
Un système complet devrait se poser simultanément toutes ces questions pour prendre la meilleure décision et tenir compte à la fois du passé (toutes les décisions et leurs conséquences antérieures), du présent (tous les paramètres actualisés) et de l'avenir (conditionnel à toutes les décisions acceptables du moment).

Ce modèle est très riche et il offre un potentiel qui dépasse largement les limites que l'on peut atteindre avec nos ordinateurs conventionnels. Lorsqu'on désire programmer le modèle de Ouellet, le principal problème que l'on rencontre est la gestion simultanée des informations provenant de tous les ensembles. Une programmation parallèle serait sans doute préférable, mais nous utiliserons plutôt une approche séquentielle. Le questionnement sera limité à un ordre prédéfini et nous développerons les niveaux de règles de gestion afin de respecter l'esprit du modèle de Ouellet.

\section{2: Le modèle global}

Considérons le schéma suivant.

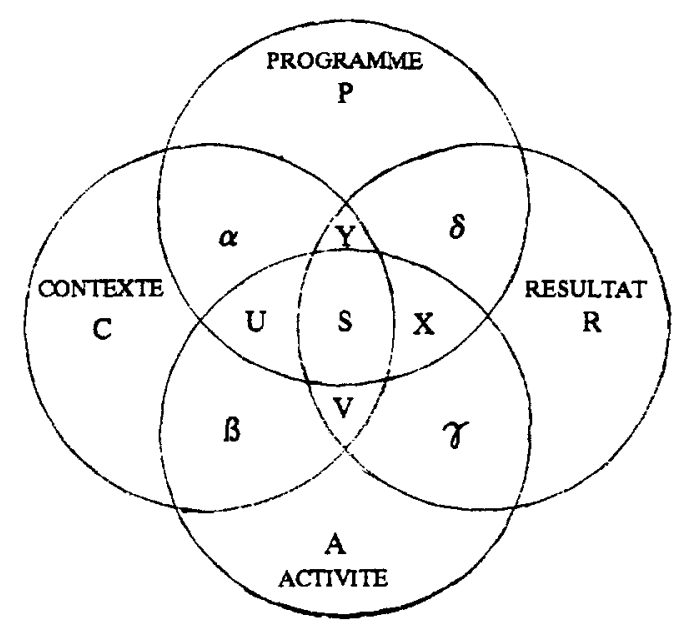

FIGURE 3.4 : Structuration des règles pour un système à 4 bases. 
Ce schéma suggère une structuration de règles reliant les quatre bases de connaissances (ou de faits). Les lettres $\alpha, \beta, \gamma, \delta, U, V, X$ et $Y$ désignent les zones d'interaction entre ces bases. Les lettres grecques $\alpha, \beta, \gamma$ et $\delta$ désignent les règles de gestion impliquant les bases prises deux à deux et les lettres $U, V, X$ et $Y$ représentent les règles de gestion d'un niveau plus élevé impliquant les bases prises trois à trois. Au centre, $S$ désigne la partie du superviseur qui, en situation d'impasse, fera appel à un expert à l'externe s'il est incapable d'équilibrer le système à partir des connaissances qu'il possède.

Du modèle de Ouellet, on tire quatre bases de connaissances nécessaires et suffisantes pour assurer un bon fonctionnement du système. Après une initialisation du système au début d'une session d'apprentissage (Contexte et Activité) et après avoir déterminé l'objectif (Programme et Résultat), il faut maintenant s'assurer que le système agisse selon une stratégie adéquate que nous allons maintenant préciser.

\section{3: La stratégie retenue}

Woodruff [53] présente le schéma suivant comme modèle de l'apprentissage ou, plus explicitement, du processus cognitif de l'élève en situation d'apprentissage. 


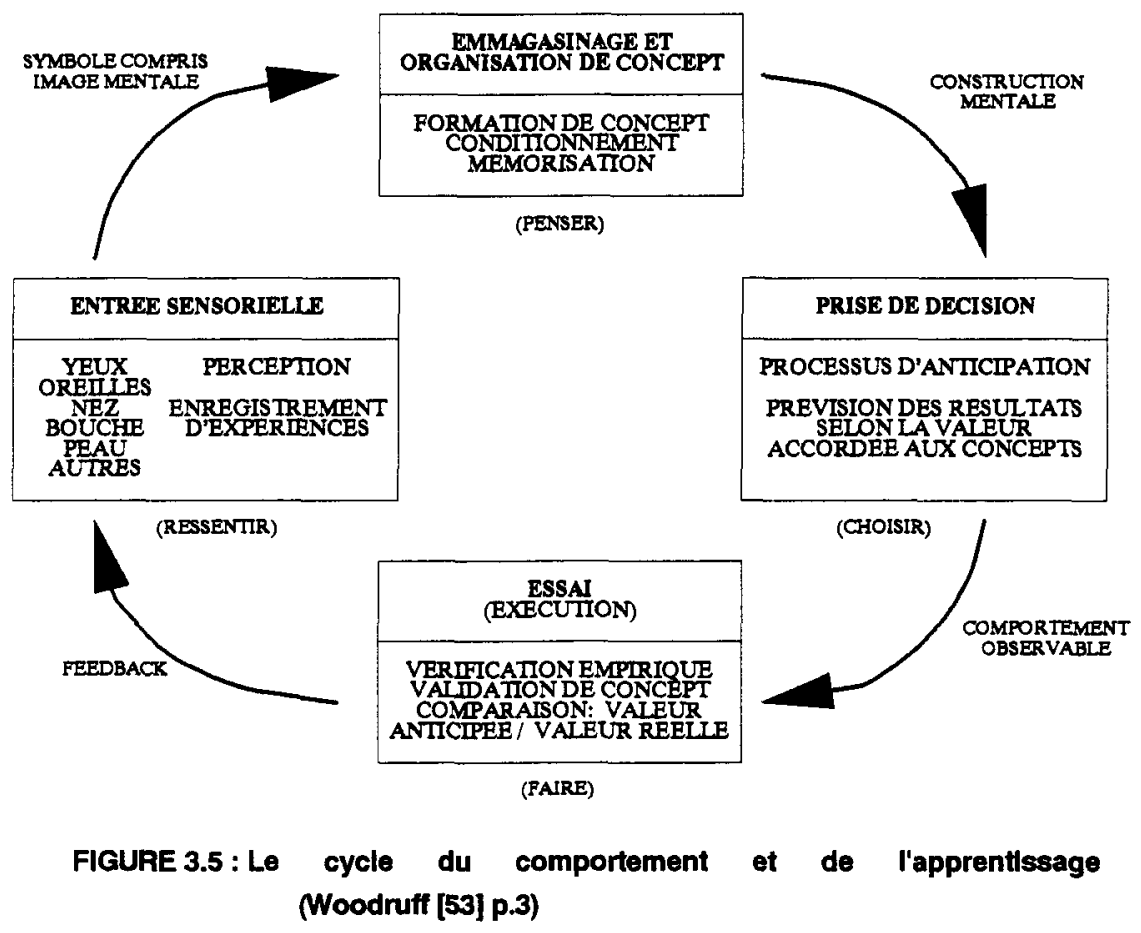

Un lien peut être fait entre le modèle de Woodruff et celui de Ouellet. On peut en effet établir une correspondance entre les entrées sensorielles de Woodruff et le contexte de Ouellet. L'emmagasinage et organisation de concept est associé à l'intervention pédagogique de Ouellet (le programme). La prise de décision correspond aux résultats et finalement l'essai aux processus d'apprentissage (activités).

Remarquons que ces deux visions sont, d'une certaine façon, complémentaires. Woodruff s'adresse au cycle du comportement de l'apprenant tandis que Ouellet traite la démarche du tuteur dans une situation d'apprentissage. Ouellet n'identifie pas de cycle particulier dans une situation d'apprentissage mais spécifie quatre ensembles en continuelle interaction. La progression cyclique proposée par Woodruff suggère par conséquent une approche possible pour déterminer un cycle particulier de progression qui serait potentiellement valable dans le processus d'apprentissage de Ouellet. 
Etant donné que le cycle de Woodruff représente le processus interne d'apprentissage de l'apprenant et que le système tutoriel doit, pour sa part, provoquer et adapter l'apprentissage, il semble adéquat d'adopter pour le système le cycle inverse de progression comme base de la stratégie d'apprentissage. Ceci aura pour effet de forcer l'exécution de certaines activités et de provoquer des résultats conformes aux objectifs visés.

\section{4: Base de l'algorithme du système}

Le système progresse à partir d'un programme à réaliser (objectif d'apprentissage à compléter) et d'un contexte particulier. On espère un certain résultat et on cherche à définir les activités adéquates. On tente ainsi d'agir pour qu'il y ait un réel apprentissage tout en respectant le cycle naturel de l'apprenant.

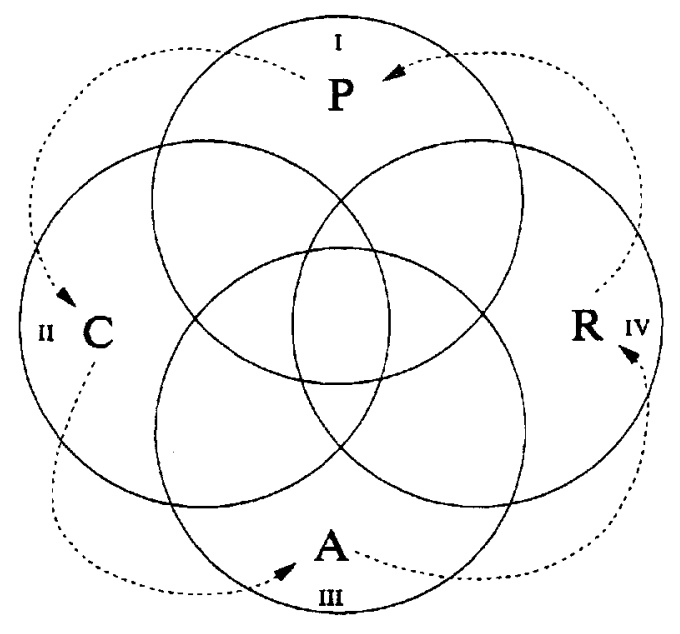

FIGURE 3.6:Mode de progression

L'apprenant réalise ses apprentissages à l'aide d'activités contenues dans la base Activité, aussi faut-il qu'elles soient pertinentes. Il est par conséquent naturel de se servir du programme et du contexte pour déterminer l'activité 
adéquate à proposer à l'apprenant. Voici donc brièvement le fonctionnement du système présenté:

Etape 1: Initialisation de la base PROGRAMME.(niveau 0 de règles)

Question 1: Quel est l'objectif visé?

A l'arrivée d'un nouvel usager inconnu du système, il y a initialisation des bases. On défini alors un programme global $\left(P_{0}\right)$ pour l'instant 0 . Cette base peut être initialisée par un expert extérieur, par un système expert ou encore par le système lui-même en utilisant un pré-test. Cette initialisation permet de définir un premier chemin critique d'objectifs (ou liste de concepts chaînés) dans la base Programme. On convient d'appeler cette liste d'éléments de programmes, le programme global pour l'instant $0\left(P_{0}\right)$. De cet ensemble $P_{0}$ on choisira l'élément de programme le plus pertinent po qu'on conviendra de nommer le programme local pour l'instant 0 .

Etape 2: Initialisation de la base CONTEXTE.(Niveau 0 de règles)

Question 2: Qui est l'apprenant?

On initialise le contexte global pour l'instant zéro $\left(\mathrm{C}_{0}\right)$ en assignant une norme ou degré de pertinence à l'aide du profil psychologique de l'usager pour chacun des éléments de contexte représentant le ou les canaux de communication privilégiés de l'apprenant. De cet ensemble de contextes, on choisira l'élément le plus pertinent pour l'instant 0 qu'on note $c_{0}$.

Etape 3: Choix d'une activité. (Niveau 1 de règles)

Question 3: Quel est l'activité la plus efficace selon l'objectif (déterminé à l'étape 1) et le contexte (déterminé à l'étape 2)?

Le système fixe la base Activité en faisant correspondre une norme locale à chaque activité possible. Cette norme est établie par rapport au programme local $(\mathrm{po})$ et contexte local $(\mathrm{co})$ déjà définis. La règle $\alpha$ de niveau 1 , intervenant à ce stade, permet de définir si possible une activité globale $\left(A_{0}\right)$ : un ensemble 
d'activités possibles. De cet ensemble, une activité locale (ao) sera choisie et soumise à l'usager.

Etape 4: Choix d'un résultat.(Niveau 1 de règles)

Question 4: Quel élément de résultat faut-il espérer compte tenu du contexte considéré (déterminé à l'étape 2) et de l'activité choisie (déterminée à l'étape 3)?

L'évaluation se fait lors de l'utilisation de la règle $\beta$ de niveau 1 en définissant un résultat global $R_{0}$ (un ensemble d'éléments de résultats) à partir des normes du contexte local et de l'activité locale choisis aux étapes précédentes. A partir de cet ensemble de résultats compatibles $R_{0}$, on choisira un résultat local ro.

Etape 5: Choix d'un programme.(Niveau 1 de règles)

Question 5: Quel est le nouveau programme à considérer selon l'activité retenue précédemment (étape 3) et le résultat obtenu (étape 4)?

On fait appel à la règle $\gamma$ de niveau 1 afin de définir un nouveau programme global $P_{1}$ (ou chemin critique) à partir des normes de l'activité locale et du résultat local choisis. Puis on choisira un nouvel élément de programme local $p_{1}$ à l'intérieur de cet ensemble de programmes compatibles.

Etape 6: Choix d'un contexte. (niveau 1 de règles)

Question 6: Quel est le nouveau contexte à considérer compte tenu du résultat obtenu (étape 4) et du nouveau programme considéré (étape 5)?

On utilise la règle $\delta$ de la même façon pour obtenir un nouveau contexte global à partir du résultat local et du programme local, puis un nouveau contexte local sera choisi de cet ensemble.

Retour à l'étape 3 jusqu'à l'obtention du résultat désiré. 
On peut représenter ce processus par le schéma suivant.
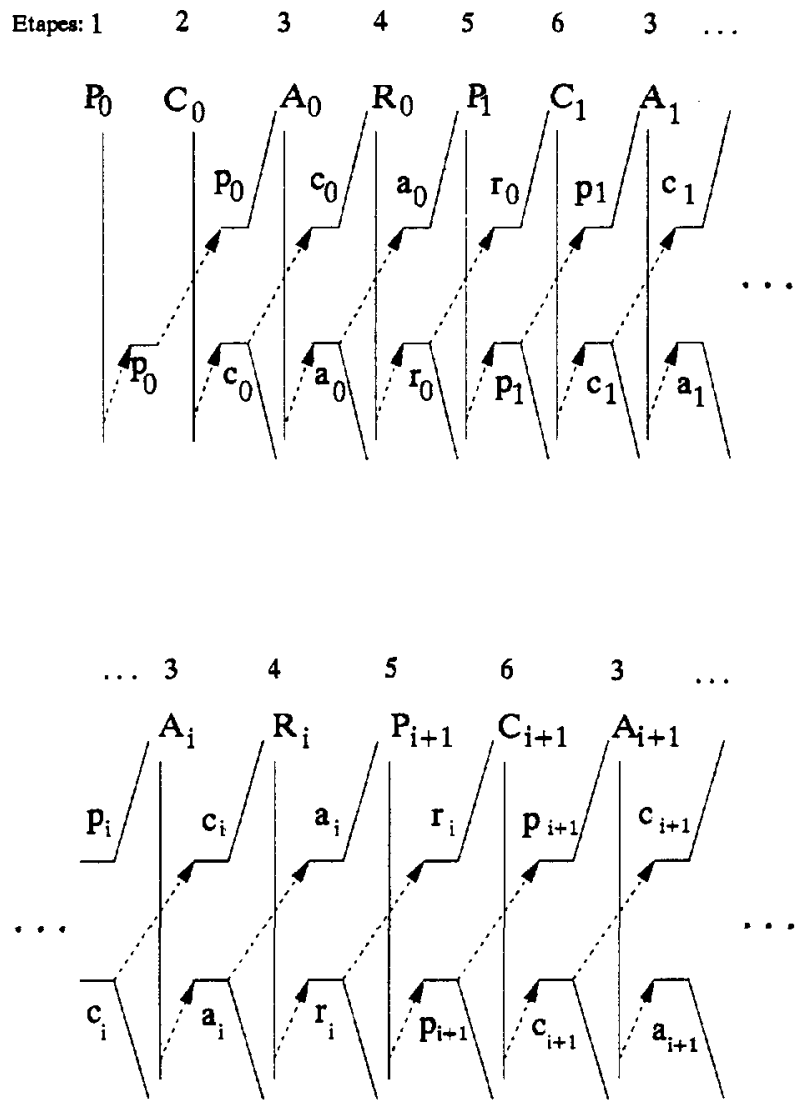

FIGURE 3.7 : Schéma fonctionnel des niveaux 0 et 1 du système

Remarquons que le système est fonctionnel en n'utilisant que ces bases et ces règles de niveau 1 mais il subsiste une possibilité de problèmes lorsqu'on obtient un ensemble de faits vide. $\mathrm{Si}$, par exemple, $\mathrm{A}$ (un ensemble d'activités compatibles) est vide, on se trouve dans l'impossibilité de choisir une activité locale, d'où l'impasse. Les règles de niveaux 2 et 3 servent à corriger la situation lorsque les règles de niveau 1 ne permettent pas de poursuivre la formation.

En fait, tant que le système peut définir des faits globaux en prenant les bases deux à deux et qu'il peut trouver un fait local satisfaisant à chaque étape, 
tout va très bien. Dans le cas contraire, il faut utiliser les règles de niveau 2 , et, si l'impasse persiste, on passe au niveau 3, c'est-à-dire au niveau du superviseur qui, finalement, fera appel à l'expert humain s'il ne trouve pas à dénouer la situation. Ce qui nous mène au schéma fonctionnel suivant que nous appellerons "la chenille" à cause de son allure.
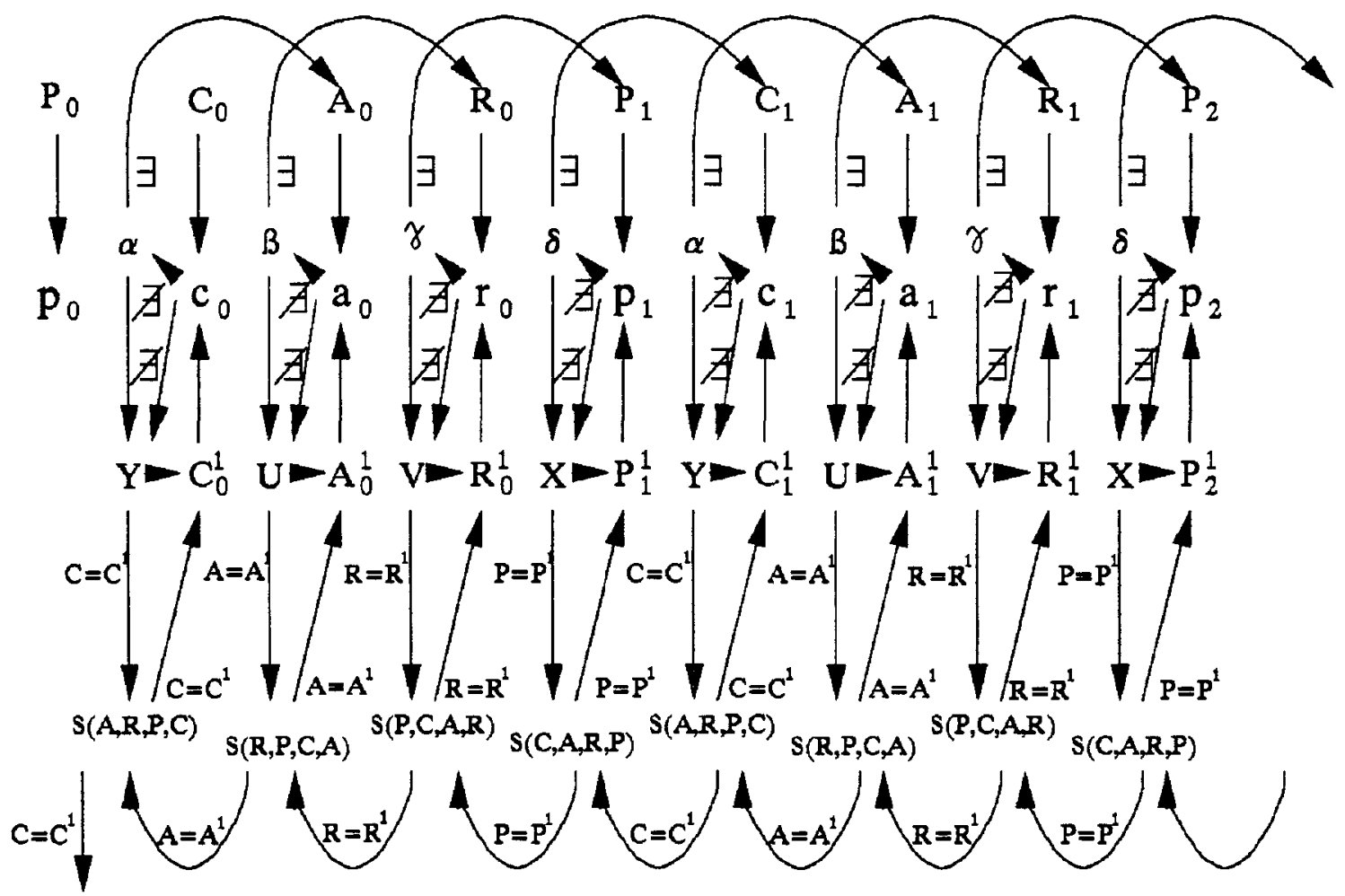

Réinitialisation

FIGURE 3.8 : La chenille ou schéma fonctionnel du système global

Dans ce schéma, on a:

$\mathrm{P}_{\mathrm{i}}=$ le programme global à l'instant $\mathrm{i}$

$\mathrm{C}_{\mathrm{i}}=$ le contexte global à l'instant $\mathrm{i}$

$A_{i}=$ l'activité globale à l'instant $i$

$R_{i}=$ le résultat global à l'instant $i$

$\mathrm{p}_{\mathrm{i}}=$ le programme local à l'instant $\mathrm{i}$

$c_{i}=$ le contexte local à l'instant $i$

$a_{i}=$ l'activité locale à l'instant $i$ 


$$
\begin{aligned}
& \mathrm{r}_{i}=\text { le résultat local à l'instant } \mathrm{i} \\
& \mathrm{P}_{\mathrm{i}}=\text { le programme global modifié } \\
& C^{\prime}{ }_{i}=\text { le contexte global modifié } \\
& A_{i}^{\prime}=\text { l'activité globale modifiée } \\
& \mathrm{R}_{\mathrm{i}}=\text { le résultat global modifié } \\
& \alpha=\text { règle de niveau } 1 ; \alpha\left(p_{i}, c_{i}\right)=A_{i} \\
& \beta=\text { règle de niveau } 1 ; \beta\left(c_{i}, a_{i}\right)=R_{i} \\
& \gamma=\text { règle de niveau } 1 ; \gamma\left(a_{i}, r_{i}\right)=P_{i+1} \\
& \delta=\text { règle de niveau } 1 ; \delta\left(r_{i}, p_{i+1}\right)=C_{i+1} \\
& U \text { = règle de niveau } 2 ; U\left(P_{i}, C_{i}, A_{i}\right)=A_{i}^{\prime} \\
& V=\text { règle de niveau } 2 ; V\left(C_{i}, A_{j}, R_{i}\right)=R_{i}^{\prime} \\
& X=\text { règle de niveau 2; } X\left(A_{i}, R_{i}, P_{i+1}\right)=P^{\prime}{ }_{i+1} \\
& Y=\text { règle de niveau 2; } Y\left(R_{i}, P_{i+1}, C_{i+1}\right)=C_{i+1}^{\prime} \\
& S=\text { règle de niveau } 3 \text {; } \\
& S\left(A_{i-1}, R_{i-1}, P_{i}, C_{j}\right)=C_{i} \\
& S\left(R_{i-1}, P_{j}, C_{j}, A_{j}\right)=A_{i}^{\prime} \\
& S\left(P_{i}, C_{j}, A_{j}, R_{i}\right)=R_{i}^{\prime} \\
& S\left(C_{j}, A_{i}, R_{i}, P_{i+1}\right)=P_{i+1}^{\prime}
\end{aligned}
$$

A chaque fois que le système est incapable de définir un fait local au niveau 1 (avec les règles $\alpha, \beta, \gamma$ et $\delta$ ), il fait appel au niveau 2 dans le but de définir un nouveau fait global. Si le fait global trouvé au niveau 2 est différent de celui trouvé au niveau 1, alors le système l'adopte et poursuit au niveau 1. Si, par contre, le fait global trouvé au niveau 2 est identique à celui du niveau 1, alors le problème persiste puisqu'on ne pouvait trouver de fait local avec ce dernier, le système passe au niveau 3.

Au niveau 3, le superviseur a pour but d'identifier la source du problème, d'essayer de le résoudre ou encore de questionner à l'externe où on peut faire appel soit à un système expert spécialisé dans le domaine de la base concernée, soit à un expert humain. Lorsque la réponse au superviseur permet de trouver un fait global différent du précédent, on poursuit la démarche au niveau 1 avec ce 
nouveau fait global. Si cette démarche externe aboutit au même résultat global, le superviseur passe au questionnement dans le champ d'expertise des autres bases.

\subsection{Généralisation du modèle}

Comme la situation d'apprentissage représente un problème de gestion parmi les plus complexes, il sera facile d'étendre son utilisation a des cas de différentes dimensions. Par exemple, en robotique, pour un problème comme la gestion d'un bras manipulateur, la quatrième base (Contexte) n'est plus nécessaire et on peut utiliser un système à trois bases, l'une d'entre elles se trouvant dégénérée.

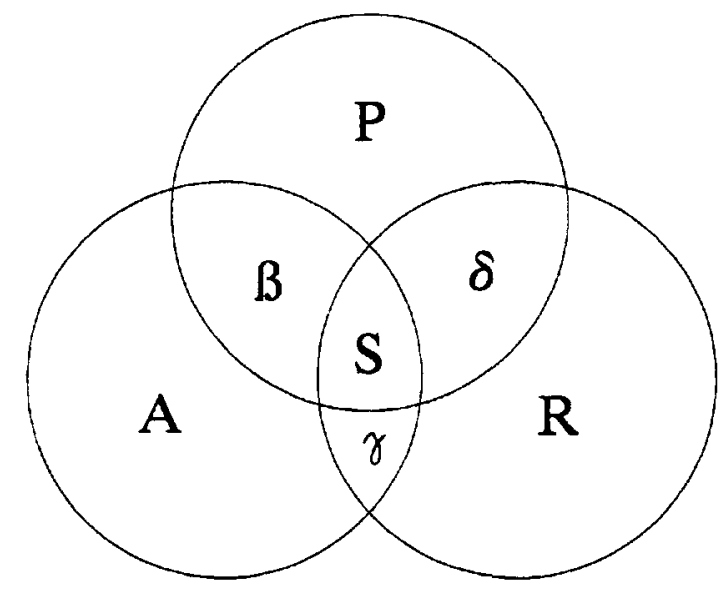

FIGURE 3.9: Structuration des règles pour un système à 3 bases.

On peut, dans d'autres situations, considérer cinq bases au lieu de quatre. L'algorithme utilisé s'adaptant à un nombre quelconque de bases, il est possible de considérer un système tout à fait identique mais comprenant $n$ bases de connaissances où $n$ peut prendre toutes les valeurs entières supérieures à 2 . 


\subsection{Conclusion}

La présentation du modèle général a permis d'obtenir un mode de progression en accord avec le modèle présenté par Ouellet. Notre système peut donc gérer efficacement une situation d'apprentissage.

Comme on l'a vu, on aurait pu considérer cinq bases au lieu de quatre mais, dans un contexte d'apprentissage, l'apport de cette cinquième base ne semble pas nécessaire d'après les spécialistes en éducation. Toutefois, la porte n'est pas fermée car la structure du modèle est très souple de sorte qu'il fonctionnerait exactement de la même façon s'il comportait un nombre quelconque de bases.

Le mode de progression utilisé ici demeurant le même pour tous les cas, qu'il s'agisse de la gestion d'une situation d'apprentissage ou non, on a obtenu une structure ouverte qui permet des ajouts subséquents et des modifications des objets de connaissances. 


\section{CHAPITRE IV}

\section{LES REGLES DE GESTION}




\section{CHAPITRE IV}

\section{LES REGLES DE GESTION}

\section{0: Introduction}

L'algorithme choisi au chapitre précédent pour gérer la situation qui nous intéresse nécessite l'utilisation de plusieurs niveaux de règles en plus d'une gestion globale du système assurée par un superviseur. Nous obtenons ainsi un système cyclique comportant des règles d'action à différents niveaux soit:

- au niveau du superviseur pour gérer le système entier;

- au niveau 0 pour gérer les quatre (4) systèmes experts représentés par chacune des bases de connaissances prises séparément;

- au niveau 1 pour permettre le passage d'une base à l'autre (mode de progression) afin de les modifier à tour de rôle (adaptabilité);

- au niveau 2 pour gérer les cas où le niveau 1 a conduit à une impasse parce qu'un des objets retenus dans les bases de connaissances précédentes a été mal choisi. Si ce niveau de règles ne résout pas le problème, nous remettrons le cas au superviseur qui contrôle le système entier et qui a le pouvoir de demander de l'aide extérieure;

- au niveau (-1)ce qui entraîne une rétroaction sur une base de connaissance; et

- au niveau (-2) ce qui entraîne une rétroaction sur deux bases de connaissances. 
Dans le présent chapitre, nous étudierons les fonctions permettant les gestions à ces différents niveaux de règles en nous basant sur la théorie des systèmes de Le Moigne [28].

\section{1: Référentiel de Le Moigne (Référentiel TEF)}

"En changeant ce qu'il connaît du monde l'homme change le monde qu'il connaît; En changeant le monde dans lequel il vit, l'homme se change lui-même."

Emprunté par Le Moigne ([28] p.58)

à Th.DOBSZHANSKY (1961-1966 p.391)

Cette phrase en dit long sur l'humain et s'applique bien a l'éducation. Elle représente également très bien le fondement du système dont il est question ici. En effet, on pourrait la traduire comme suit:

"En changeant ce qu'il connaît de l'apprenant, le système change l'apprenant qu'il connait; En changeant l'apprenant, le système se change lui-même."

Le système proposé est adaptatif puisqu'il améliore, étape par étape, sa connaissance de l'usager apprenant de façon à répondre de mieux en mieux à ses besoins de formation.

Globalement le système prend un apprenant à un certain point (1) de sa formation au début de l'apprentissage et tente de l'amener à un autre point (2) (voir 
figure 4.1); le point (2) représentant le point où on peut considérer que l'apprenant a acquis une connaissance suffisante du ou des concepts à acquérir.

Le Moigne [28], dans sa définition du système général, nous dit qu'un tel processus se définit comme un ensemble ordonné de changements affectant la position d'une famille d'objets dans le temps, dans l'espace et dans leur forme et qu'on peut le représenté par un référentiel que nous appellerons TEF: $T$ pour temps, $E$ pour espace et $F$ pour forme (figure 4.1).

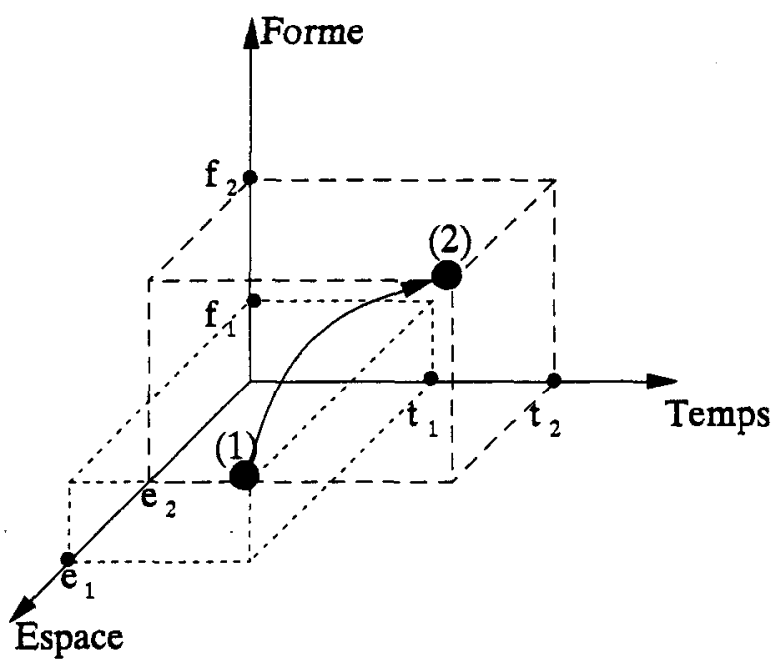

FIGURE 4.1 : Référentlel TEF (Temps, Espace, Forme) permettant de repérer la positton des objets soumls à des processus. (Le Molgne [28] p.63)

Notre système représente un processus de transformation affectant l'apprenant à travers un Programme global, un Contexte global, une Activité globale et un Résultat global. Par analogie au référentiel TEF, nous pouvons le définir à l'intérieur d'un référentiel PCAR (Programme, Contexte, Activité, Résultat) à quatre dimensions. 


\section{2: Les référentiels}

Un tel référentiel (à quatre dimensions) est impossible à représenter schématiquement, aussi devons-nous séparer la transformation générale en plusieurs groupes de changements ordonnés à l'intérieur de sous-référentiels comme:

PCA : les sous-référentiels Programme-Contexte-Activité où seront modifiés les degrés de pertinence de chacune des activités de l'ensemble Activité;

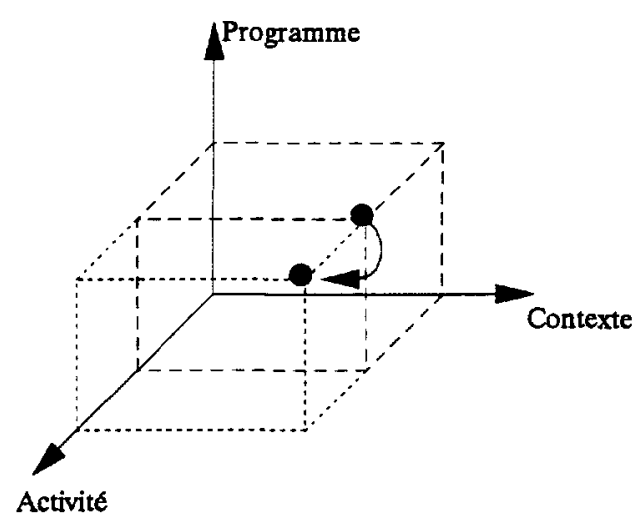

FIGURE 4.2: Sous-référentiel PCA (Programme-Contexte-Actlvité)

Nous obtenons ainsi des sous-référentiels $X Y Z$ où $X, Y$ et $Z$ représentent les trois premiers éléments de toute permutation cyclique de $P, C, A$ et $R$. C'est-àdire XYZ $\varepsilon\{P C A, C A R, A R P, R P C\}$.

Remarquons que notre référentiel PCAR est formé de plusieurs sousréférentiels de chaque type. Ainsi, si $P, C, A$ et $R$ représentent respectivement le nombre d'éléments de chacune des bases correspondantes, nous sommes en présence de:

$$
\mathrm{P} \times \mathrm{CxA}+\mathrm{C} \times \mathrm{AxR}+\mathrm{AxR} \mathrm{xP}+\mathrm{RxPxC}
$$

sous-référentiels tous en continuel changement. Chaque référentiel représente un ensemble d'éléments descriptifs de l'état de l'apprenant. 
Ces référentiels permettent une évaluation continue des différents éléments de chacune des bases offrant du même coup la possibilité d'évaluer l'avancement général de la situation moyennant des règles de gestion efficaces.

Au chapitre 3 , nous avons mis en évidence la nécessité de différents niveaux de règles en nous guidant du schéma suivant:

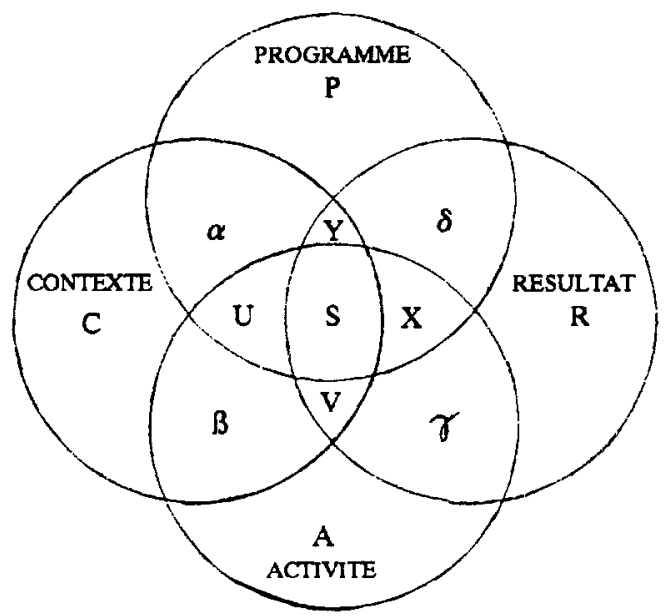

où:

- $P, C, A$ et $R$ représentent chacune des bases et les règles de niveau 0 agissant sur chacune d'elles;

$-\alpha, \beta, \gamma$ et $\delta$ représentent des règles de gestion de niveau 1 et concement l'interaction des bases prises deux à deux;

- U, V, X et $Y$ représentent des règles de niveau 2 et concernent l'interaction des bases prises trois à trois; et

- $S$ représente le superviseur au niveau qui gère le système global et agit sur les quatre bases à la fois.

FIGURE 4.3: Structuration des règles pour un système à 4 bases

Ces règles visent à guider adéquatement l'apprenant dans le monde des connaissances que nous lui offrons. 


\section{3: Degrés de pertinence et normes de compatibilité}

\subsection{1: Degrés de pertinence}

Idéalement, chaque base est alimentée de connaissances et gérée par des systèmes experts d'acquisition. Ces connaissances demandent d'être représentées. Nous limiterons cette représentation à un degré de pertinence associé à chaque fait de chacune des bases. Ces degrés de pertinence permettent au système de choisir le fait le plus pertinent pour chaque situation. Les règles du niveau 0 reflètent ainsi la pertinence des éléments de connaissances de chacune des bases dans la situation effective d'apprentissage.

Au départ, chaque degré de pertinence est précisé par un expert. Par exemple, la base Programme comprenant un certain nombre d'objectifs à atteindre, un expert externe aura préalablement déterminé un premier objectif local à atteindre ainsi qu'un premier chemin critique permettant l'acquisition de l'objectif global.

Par la suite, les degrés de pertinence de chaque fait de chacune des bases sont modifiés lors des passages aux niveaux supérieurs de règles. Les modifications sont fonction du comportement de l'apprenant lors de l'apprentissage. Cette correction permet au système d'ajuster ses décisions à chaque tour du cycle selon les résultats obtenus. 


\subsection{2: Normes de compatibilité}

Les règles de niveaux 1 et 2 sont basées sur des normes de compatibilité entre les bases prises deux à deux. Le calcul des nouveaux degrés de pertinence ne dépendent pas seulement de la base concernée, mais également des deux bases qui le précèdent. Plus exactement, nous avons besoin, dans nos calculs, d'utiliser les normes de compatibilité des faits de la base active avec ceux des deux bases qui la précèdent directement.

Ainsi, pour faire les calculs de degrés de pertinence des éléments de la base Activité, nous utiliserons pour chaque activité:

1- la norme de compatibilité de l'élément de programme retenu avec l'activité en question,

2- la norme de compatibilité de l'élément de contexte retenu avec la même activité.

Ces normes, précisées par des experts, forment des grilles comme les suivantes:

\begin{tabular}{c|ccccc} 
ai $p k \mid$ & $\mathrm{p} 1$ & $\mathrm{p} 2$ & $\mathrm{p} 3$ & $\mathrm{p} 4$ &.. \\
\hline $\mathrm{a} 1$ & 1 & 0 & 0 & 0 \\
$\mathrm{a} 2$ & 0 & 0 & 1 & .8 & \\
$\mathrm{a} 3$ & 0 & 0 & .8 & 0 \\
$\mathrm{a} 4$ & 0 & 0 & .8 & 0 \\
a5 & 0 & 0 & .8 & 0 \\
$\ldots$ & & & &
\end{tabular}

\begin{tabular}{c|ccccc} 
ai c1 & $\mathrm{c1}$ & $\mathrm{c} 2$ & $\mathrm{c3}$ & $\mathrm{c4}$ &.. \\
\hline $\mathrm{a} 1$ & 0 & .5 & 1 & 0 & \\
$\mathrm{a} 2$ & .1 & .7 & 1 & .1 & \\
$\mathrm{a} 3$ & .2 & .7 & 1 & .2 & \\
$\mathrm{a} 4$ & .2 & .7 & 1 & .2 & \\
$\mathrm{a} 5$ & .2 & .7 & 1 & .2 & \\
$\ldots$ & & & & &
\end{tabular}

On peut interpréter ces normes de compatibilité comme des probabilités conditionnelles. Par exemple la norme $\left|a_{i j} p_{k}\right|$ peut représenter la probabilité que l'activité i présente le meilleur choix étant donné le choix préalable du programme 
k. Nous laissons toutefois à l'expert, qui précisera ces normes, le soin d'en définir une interprétation adéquate.

\subsection{Le superviseur}

Le rôle principal du superviseur est de gérer le système dans son ensemble un peu à la manière d'un système d'exploitation classique. II représente le niveau de décision le plus élevé du système et on le trouve à l'intersection des quatre ensembles. Le superviseur a accès et a le contrôle sur les quatre bases de faits. II représente le moteur d'inférence.

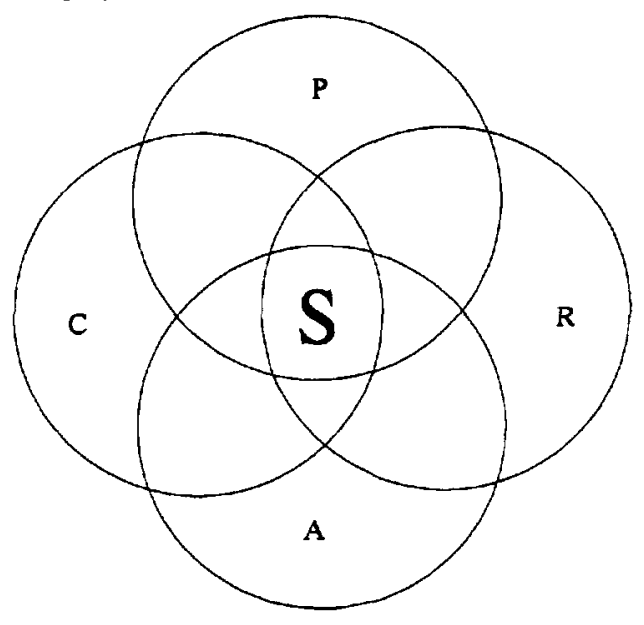

FIGURE 4.4: Supervlseur

Lors de l'initialisation du système, le superviseur donne accès à chacune des bases pour permettre l'initialisation de celles-ci. Lorsqu'un apprenant (nouveau ou ancien) prend contact avec le système, le superviseur dirige le système en provoquant le début d'une nouvelle leçon selon la connaissance qu'il a de l'apprenant. S'il y a impasse à un niveau donné, il décidera d'aller au niveau supérieur. Le superviseur gère également le retour aux différents niveaux de règles en temps et lieux. 
Les explications concernant es autres niveaux de règles (les niveaux 0,1 , 2 , (-1) et (-2)) conduiront à une meilleure compréhension du rôle du superviseur, ce dernier dirigeant le processus vers ces différents niveaux.

\section{5: Niveau 0}

Ce niveau de règles est le plus bas niveau existant dans le système puisqu'il fait intervenir une seule base à la fois. On y trouve les règles de gestion internes de chacune des bases de connaissances. Ces règles représenteraient éventuellement un système expert distinct et complètement indépendant du reste du système pour chacune des bases.

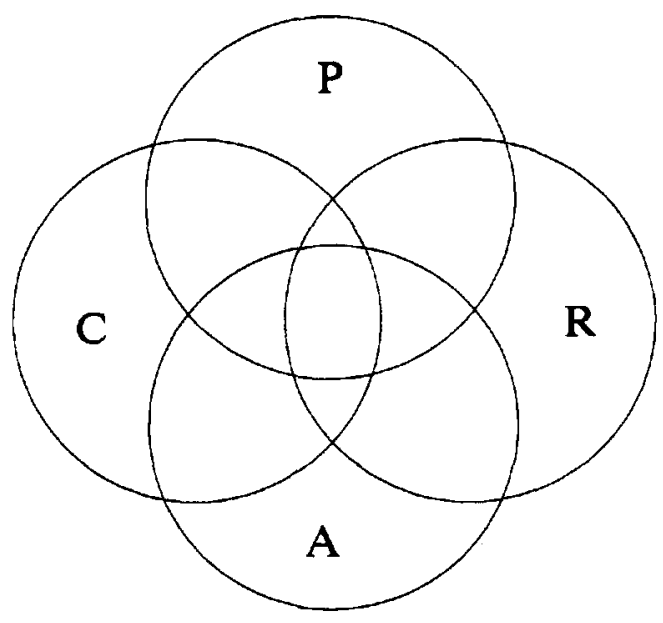

FIGURE 4.5: Structuration des règles

Les règles de ce niveau permettent de choisir, parmi un ensemble de faits compatibles, le fait le plus pertinent pour une base donnée et pour un moment donné. C'est également à ce niveau que sont gérées les interactions entre les bases de faits et l'extérieur lors des initialisations, évaluations et corrections du système. 
Parmi les règles de niveau 0 , on trouve essentiellement:

- les règles pour l'initialisation de la base Contexte (initialisation des qualités psychologiques de l'apprenant),

- les règles pour l'évaluation des activités d'apprentissage (un module demande la note obtenue pour l'activité proposée),

- certaines règles pour la modification de normes dans des cas bien précis,

- les règles pour la vérification de la réussite de l'objectif final,

- les règles pour la recherche du ou des faits les plus pertinents pour chacune des bases,

- les règles pour la recherche du fait à considérer parmi les faits compatibles,

- les règles pour le changement des limites des intervalles de compatibilité (rétrécissement et élargissement des dits intervalles).

Comme nous l'avons constaté au chapitre précédent, bien que chacune des bases de faits (c'est-à-dire les bases Programme, Contexte, Activité et Résultat) soient idéalement alimentées et gérées par des systèmes experts, nous avons besoin de règles permettant de faire progresser le système en passant d'une base à l'autre de façon cyclique tout en faisant cheminer l'apprenant jusqu'à l'objectif final. C'est ce que le système fera aux niveaux 1 et 2 de règles.

\section{6: Niveau 1}

Au niveau 1, nous trouvons des règles permettant de faire le choix d'un élément de formation d'une base donnée en tenant compte des bases deux précédentes selon l'ordre de progression du système. Par exemple, choisir une activité d'apprentissage tient compte du programme et du contexte choisis à l'étape précédente. Comme nous l'avons déjà vu dans le schéma fonctionnel global, à ce niveau, les fonctions sont du type:

$$
\alpha(p, c)=A
$$


où:

$\alpha$ est la fonction appliquée sur un élément de programme et sur un élément de contexte,

p est l'élément de programme le plus compatible ou un des éléments de programmes les plus compatibles dans le cas d'égalité,

c est un élément de contexte faisant partie de l'ensemble des contextes compatibles de la même façon,

A est le résultat de $\alpha(p, c)$ et représente un ensemble d'activités compatibles.

Les autres fonctions $(\beta, \gamma$ et $\delta$ ) se retrouvent par permutation cyclique des éléments dû au modèle retenu pour la structuration des règles.

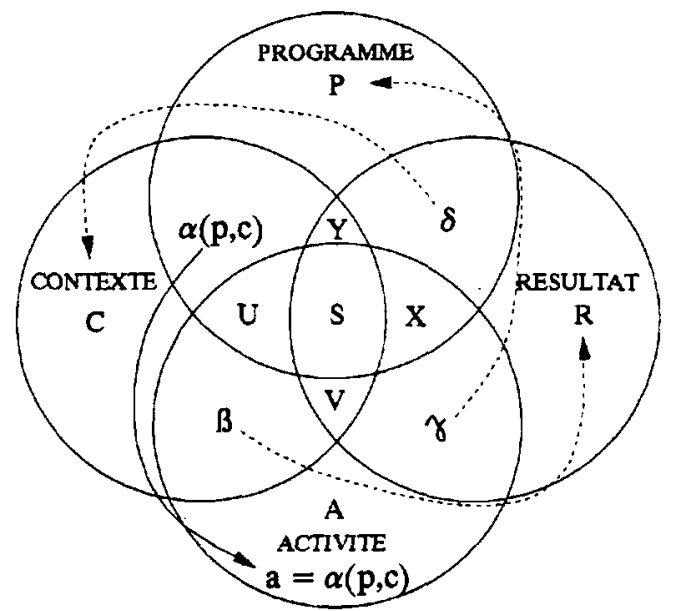

FIGURE 4.6: NIveau 1 de règles

Afin d'établir la fonction permettant le calcul de nouveaux degrés de pertinence pour les éléments de la base Activité, considérons les normes de compatibilité $\left|a_{i} p_{k}\right|$ et $\left|a_{i} c_{1}\right|$ dont nous avons discuté plus tôt. Ajoutons à cela les normes $|a i|$ et $\mid$ ai préc| où:

$|a|$ représente le degré de pertinence de l'activité i considérée au moment actuel et que nous cherchons à évaluer, et

|ai préc| représente le degré de pertinence mais à l'étape précédente de l'activité i considérée. 
Puisque toutes ces valeurs représentent des longueurs, on peut utiliser les règles de la géométrie élémentaire pour écrire le degré de pertinence de ai.

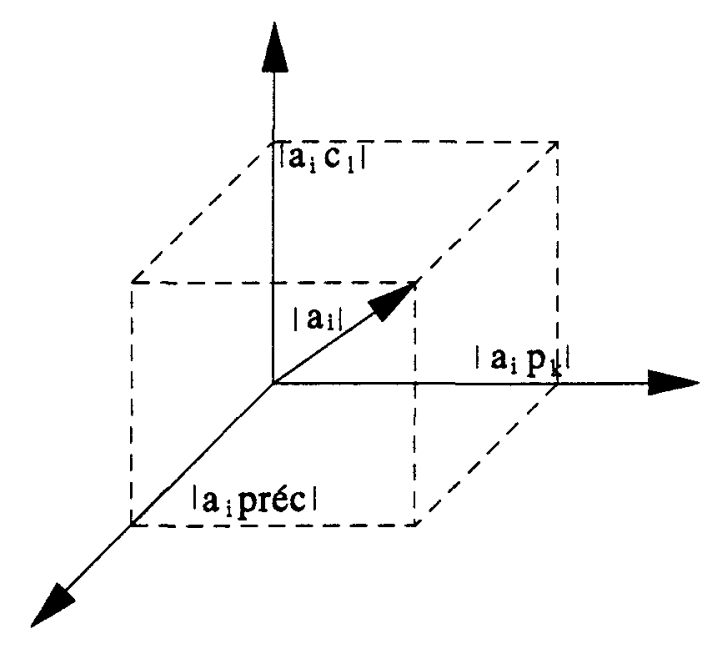

FIGURE 4.7: |ai| comme longueur du longueur résultant

En se souvenant que la longueur d'un vecteur $(x, y, z)$ dans un espace à trois dimensions est donné par:

$$
\sqrt{x^{2}+y^{2}+z^{2}}
$$

nous pouvons calculer la longueur du vecteur |ail de la façon suivante:

$$
\left.\sqrt{|a i p k|^{2}+|a i c|^{2}+|a i p r e ́ c|}\right|^{2}
$$

divisons cette longueur par $\sqrt{3}$ pour la normaliser, c'est-à-dire pour s'assurer d'obtenir un vecteur unitaire, on obtient ainsi une nouvelle norme |ai| ou degré de pertinence:

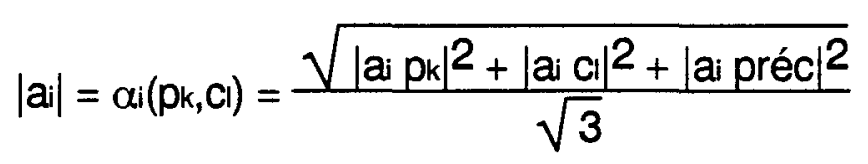

où 
$\alpha_{i}\left(p_{k}, \mathrm{Cl}\right)=$ la fonction de transfert impliquant les variables $\mathrm{pk}_{\mathrm{k}}$ et $\mathrm{cl}$

|ai $\mathrm{pk}$ = la norme de compatibilité de l'activité $\mathrm{i}$ avec le programme $\mathrm{k}$

$\mid$ ai $\mathrm{c}_{1}=$ = la norme de compatibilité de l'activité $\mathrm{i}$ avec le contexte $\mid$

|ai préc| = le degré de pertinence de l'activité i à l'étape précédente

|ai $\mid=$ le degré de pertinence calculé pour l'activité $\mathrm{i}$ à l'étape actuelle

La longueur du vecteur $|a i|$ ainsi obtenue donne le nouveau degré de pertinence de l'activité $\mathrm{i}$. Les degrés de pertinence des éléments des autres bases sont calculés de la même façon par permutation cyclique des bases. Nous obtenons ainsi les fonctions:

$$
\begin{aligned}
& \left|r_{i}\right|=\beta_{i}\left(c_{k}, a\right)=\frac{\sqrt{\left|r_{i} c_{k}\right|^{2}+\left|r_{i} a\right|^{2}+\mid r_{i} \text { préc }\left.\right|^{2}}}{\sqrt{3}} \\
& \left|p_{i}\right|=\gamma_{i}(a k, n)=\frac{\sqrt{\left|p_{i} a_{k}\right|^{2}+\left|p_{i} n^{2}+\right| p_{i} \text { préc }\left.\right|^{2}}}{\sqrt{3}} \\
& \left|c_{i}\right|=\delta_{i}\left(r_{k}, p_{l}\right)=\frac{\left.\sqrt{\left|c_{i} r_{k}\right|^{2}+\left|c_{i} p_{1}\right|^{2}+\left|c_{i} p r e ́ c\right|}\right|^{2}}{\sqrt{3}}
\end{aligned}
$$

A chaque fois, nous retenons dans un ensemble les faits jugés compatibles, c'est-à-dire ceux dont le degré de pertinence se situe entre certaines valeurs limites prédéfinies. Ensuite, nous choisissons dans l'ensemble ainsi obtenu, le fait le plus pertinent. Dans le cas d'égalité, on doit faire un choix aléatoire parmi les faits les plus pertinents.

Comme mentionné précédemment, la division par $\sqrt{3}$ permet d'obtenir une norme unitaire. Cette manière de calculer accorde une importance égale aux normes de compatibilités Activité-Programme, Activité-Contexte et au degré de pertinence précédent. Cette manière de procéder n'est pas souhaitable dans toutes les situations. Par exemple, dans le cas de la gestion d'un robot, la base Contexte s'avère complètement inutile si ce dernier a toute liberté de mouvement. 
Dans d'autres cas, il est possible qu'une des bases, sans avoir une importance nulle, soit beaucoup moins importante que les autres. Ceci pourrait se produire dans le cas de la gestion d'un projet immobilier où l'aspect des ressources humaines est moins importante que la qualité du résultat final. Ces raisons expliquent la nécessité de se donner la possibilité d'avoir une système avec des bases d'importances inégales comme nous le verrons dans la section suivante.

\subsection{1: Système avec bases d'importances inégales}

II pourrait survenir qu'une base ait moins d'importance qu'une autre ou encore, tout simplement, que les bases soient d'importance inégales. II est alors possible d'allouer un degré d'importance à chacune des bases et les fonctions de transfert sont alors généralisées comme celle-ci:

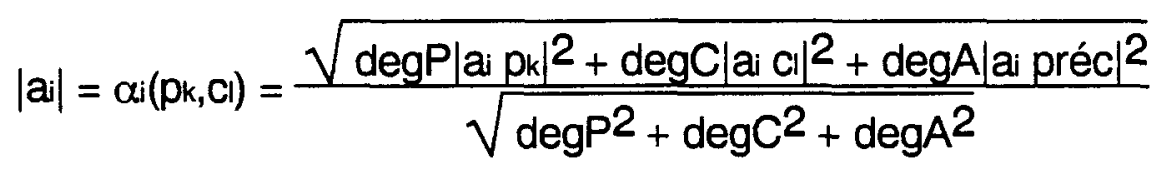

où:

degP = le degré d'importance de la base Programme dans le système $\operatorname{deg} \mathrm{C}=$ le degré d'importance de la base Contexte dans le système $\operatorname{deg} A$ = le degré d'importance de la base Activité dans le système

On trouve de cette manière des fonctions de transfert beaucoup plus souples et applicables dans plus de situations.

REMARQUE: Dans le programme informatique PROLIN dont il est question plus loin, cette forme de fonctions de transfert est utilisée. Les valeurs de degP, degC, $\operatorname{deg} A$ et $\operatorname{deg} R$ sont toutefois délibérément fixées à un (1) dans un but de simplification. II serait facile de les changer en cas de besoin à l'intérieur du programme informatique. 
Les fonctions de transfert du niveau 1 fournissent des ensembles de faits compatibles parmi lesquels on choisit le fait le plus pertinent. Si le système n'arrive pas à trouver de fait pertinent, il est clair qu'on risque d'affronter une fin abrupte des opérations, ce qui n'est nullement souhaité. Voilà pourquoi nous avons besoin de l'intervention du niveau 2 de règles.

\section{7: Niveau 2}

Le niveau 2 de règles intervient lorsqu'une fonction de transfert du niveau 1 ne peut trouver de fait pertinent. Au niveau 2, le système cherche à résoudre le problème en requestionnant les bases de façon plus complète afin de dénicher un fait pertinent.

Notons d'abord que le niveau 1 ne trouve pas de fait pertinent lorsque tous les faits d'une base ont des degrés de pertinence hors limites, c'est-à-dire à l'extérieur de l'intervalle de compatibilité. Par exemple, après le passage par le niveau 1, il est possible que toutes les normes |ai| soient hors limites, c'est-à-dire qu'on ne trouve aucune activité compatible et, assurément, aucune activité pertinente. L'activité est dite pertinente si elle est choisie par le système comme étant la plus compatible. Une activité est dite compatible si sa norme |ail se situe à l'intérieur d'un intervalle de compatibilité:

$$
\text { [ limite inf ; limite sup ] }
$$

où

"limite inf" est la limite inférieure d'acceptation du degré de pertinence et "limite sup" la limite supérieure d'acceptation.

Examinons la fonction qui a été utilisée pour le calcul des |ai| 


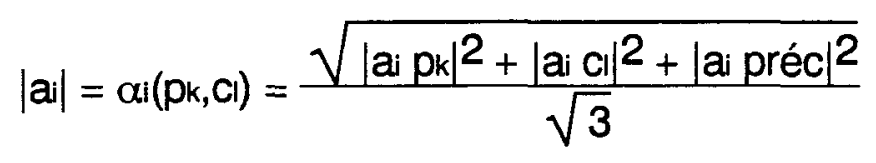

Les échecs du niveau 1 de règles peuvent se produire dans l'une des situations suivantes:

|ai $\mathrm{pk}_{\mathrm{k}} \quad$ est trop petit ou trop grand d'où l'élément de programme considéré serait mal choisi,

|ai cl| est trop petit ou trop grand d'où l'élément de contexte considéré serait mal choisi, ou encore

|ai préc| est trop petit ou trop grand, ai n'a donc aucune raison d'être choisie,

Pour nous aider à trouver une nouvelle activité pertinente (c'est-à-dire au moins une activité compatible), il faut soit:

-reconsidérer le calcul de |ai préc|,

-faire un nouveau choix d'élément de programme, ou encore

-faire un nouveau choix d'élément de contexte.

Comme il serait inutile de changer tous ces paramètres en même temps, il est résolu de n'en changer qu'un. Voici les conséquences provoquées par ces changements.

Changer la norme de lai précl:

Ce changement implique un retour en arrière trop important puisqu'il faudrait faire un retour sur quatre bases et recommencer un tour complet dont la dernière activité effectuée par l'usager (figure 4.8). 

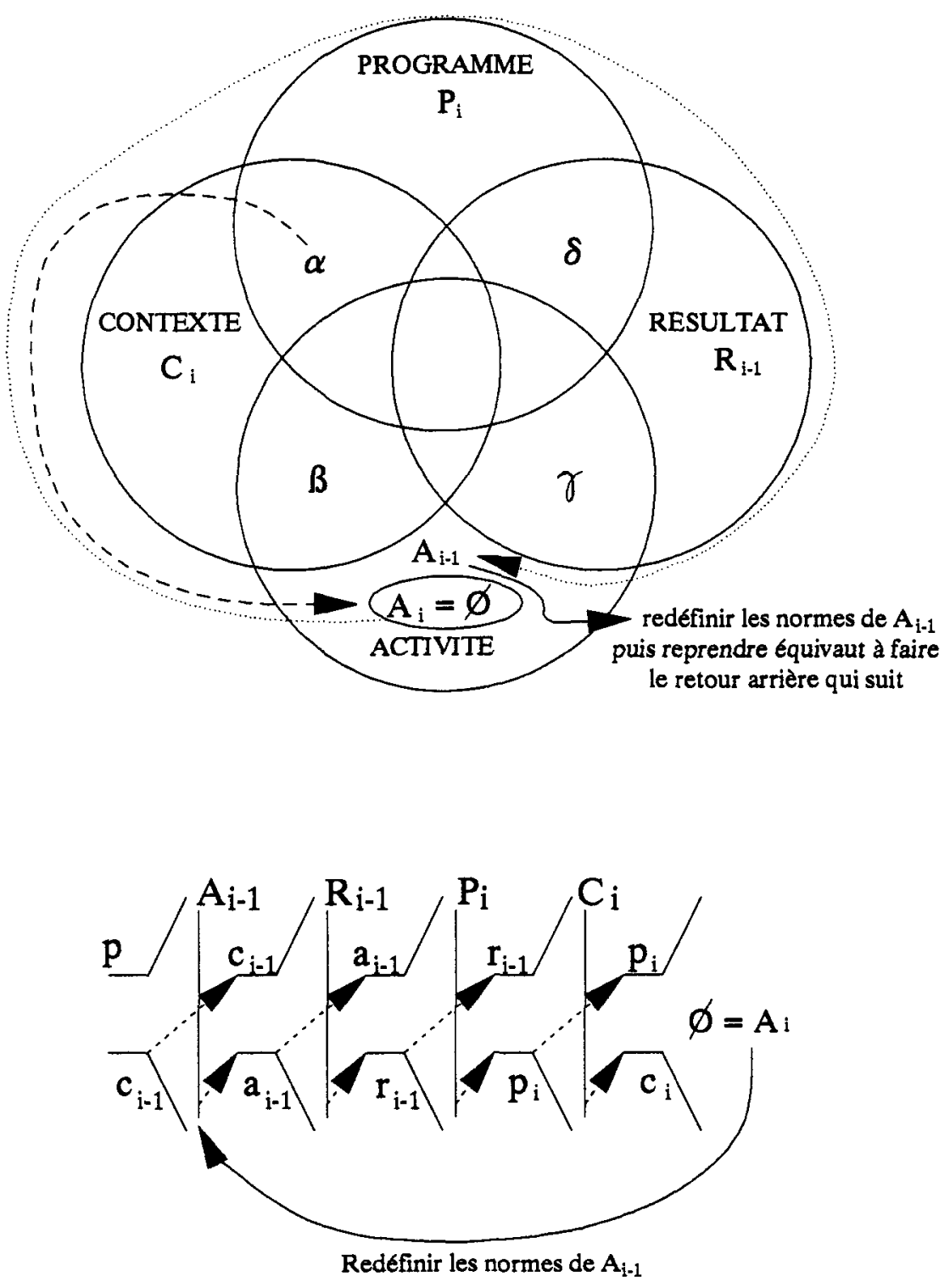

FIGURE 4.8 : Retour nécessaire sl on désire modinter la norme de |ai préc|

Ce retour n'étant pas du tout souhaitable pour l'avancement du système, il nous reste deux avenues: 
Faire un nouveau choix d'élément de programme:

Cette avenue demande de faire un retour sur deux bases pour redéfinir un nouvel élément de programme, ce qui implique également de faire un nouveau choix de contexte au retour au niveau 1 ;
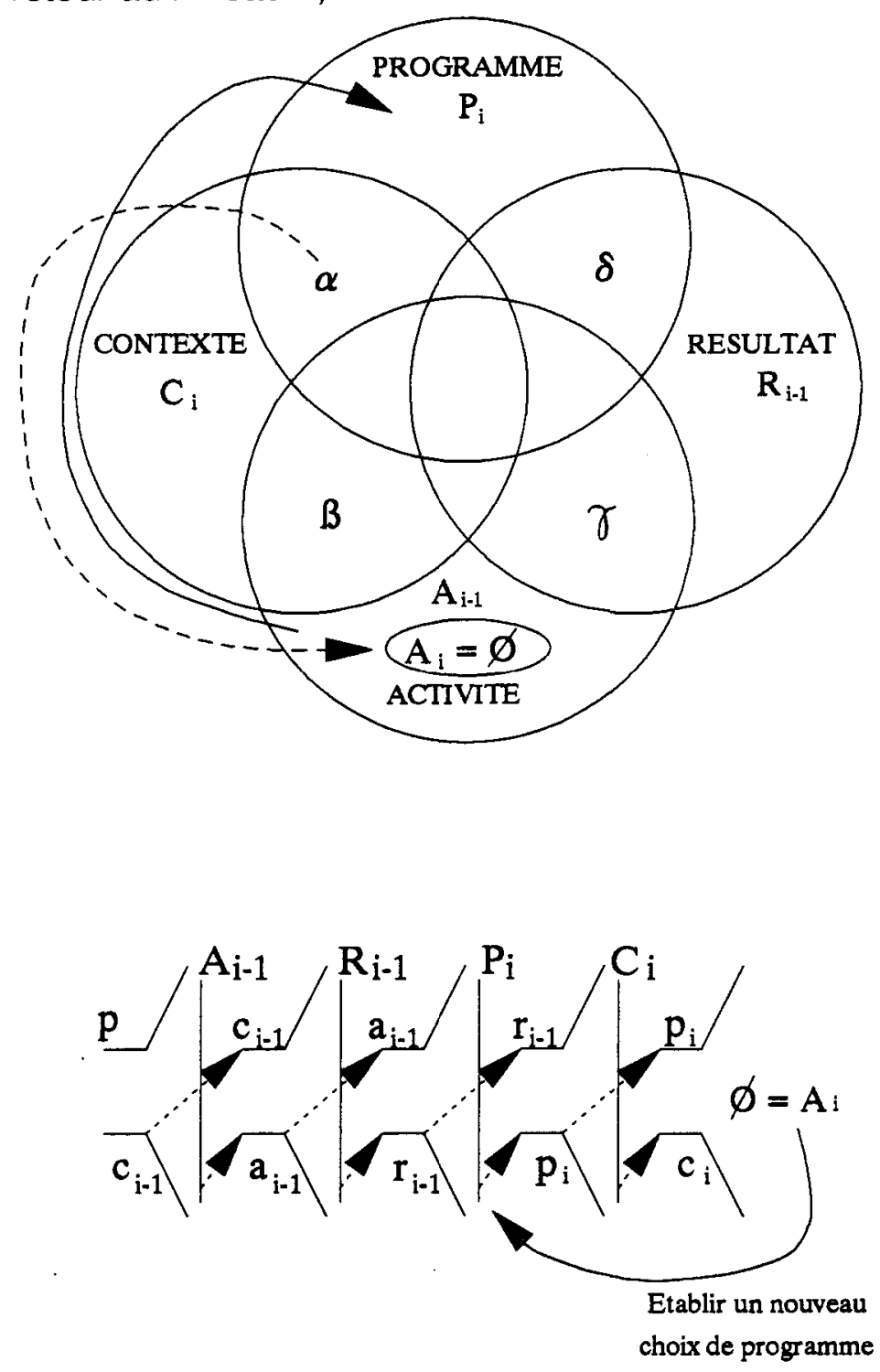

FIGURE 4.9 : Retour nécessalre pour falre un nouveau cholx de programme 
Faire un nouveau choix d'élément de contexte:

Cette avenue demande de faire un retour sur une seule base pour établir un nouveau choix de contexte. C'est ce que fait la fonction $Y i(R, P, C)$.
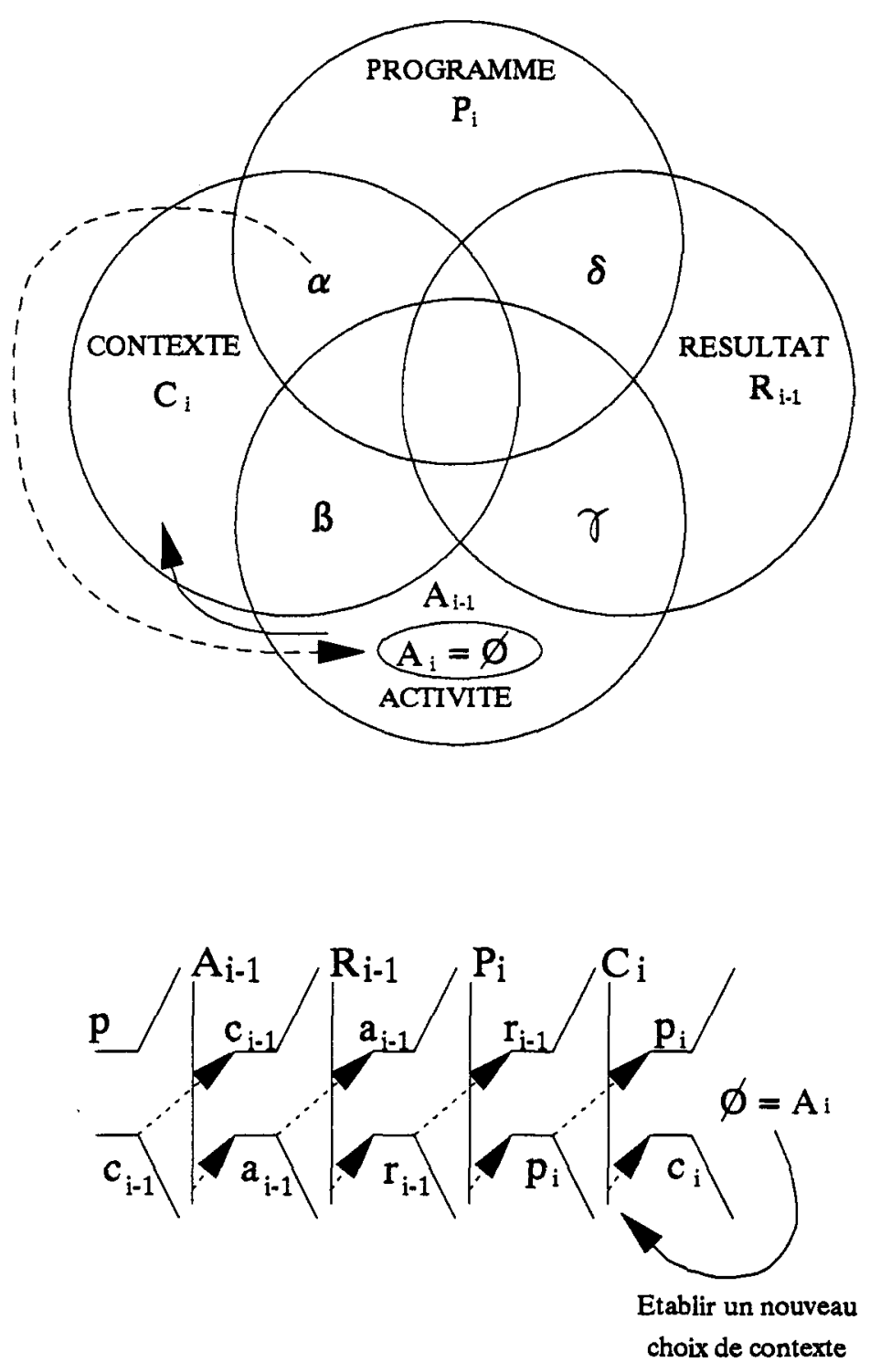
Les fonctions du niveau 2 permettent de redéfinir l'ensemble de faits compatibles de la base qui précède immédiatement celle où se situe l'impasse. Dans notre schéma de type diagramme de Venne, ces fonctions se situent aux intersections trois à trois puisqu'elles font intervenir trois bases à la fois.

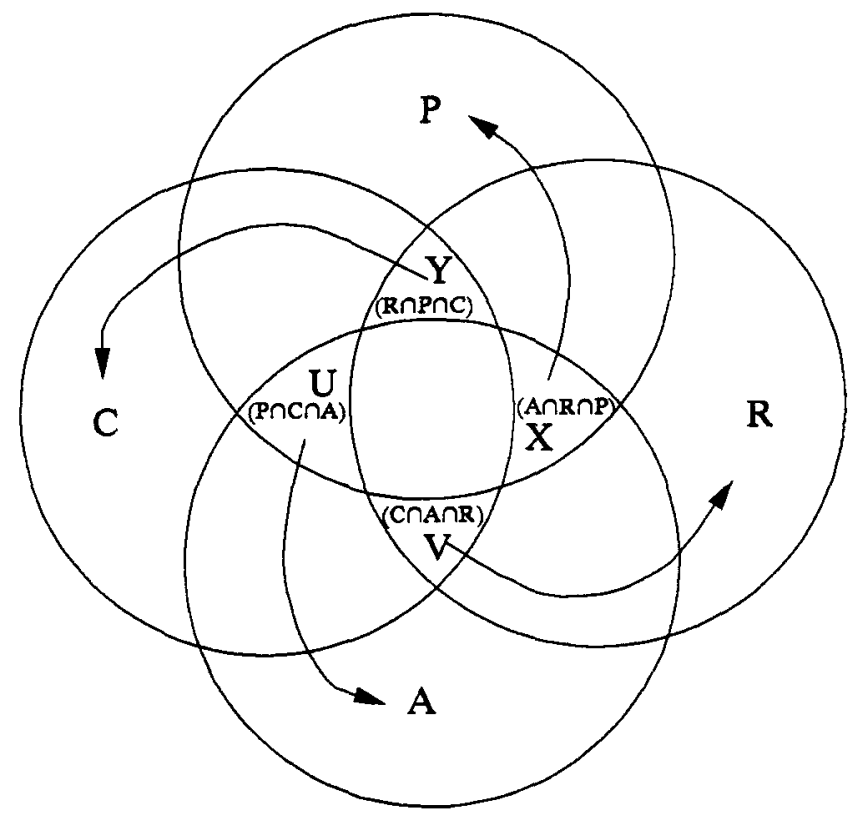

FIGURE 4.11: NIveau 2

Ainsi la fonction $Y_{i}(R, P, C)$ calcule de nouveaux degrés de pertinence pour chaque fait de la base Contexte en s'appliquant, non plus sur les faits locaux (les faits les plus pertinents $\mathrm{r}_{k}$ et $\mathrm{pl}$ ), mais sur les faits globaux (les ensembles de faits compatibles $\mathrm{R}$ et $\mathrm{P})$. 
Au niveau 2, le système recalcule les normes $\left|c_{i}\right|$ mais cette fois-ci en considérant tous les résultats compatibles et tous les programmes compatibles. II est résolu de faire la moyenne arithmétiçue de tous les résultats obtenus pour chacun des éléments de contexte d'où on obtient la fonction de niveau 2 suivante:

$$
\begin{aligned}
& \left|c_{i}\right|=Y i(R, P, C) \\
& \left|c i^{\prime}\right|=\frac{1}{n m} \sum_{k=1 \mid=1}^{n} \sum_{\sum}^{m} \frac{\sqrt{|c i r k|^{2}+|c i p|^{2}+|c i|^{2}}}{\sqrt{3}}
\end{aligned}
$$

avec

$\mathrm{R}=$ l'ensemble des atomes de résultat compatibles

$\mathrm{P} \quad=$ l'ensemble des atomes de programme compatibles

$\mathrm{C} \quad=$ l'ensemble des atomes de contextes compatibles qui seront obtenus

$\mathrm{n} \quad=$ le nombre d'atomes de résultat compatibles

$\mathrm{m} \quad=$ le nombre d'atomes de programme compatibles

$\left|\mathrm{c}_{\mathrm{i}} \mathrm{r}_{\mathrm{k}}\right|=$ la norme de compatibilité de l'atome de contexte $\mathrm{i}$ avec l'atome de résultat $k$

$\left|c_{i} p\right| \mid=$ la norme de compatibilité de l'atome de contexte i avec l'atome de programme 1

$\left|c_{i}\right|=$ le degré de pertinence de l'atome de contexte $\mathrm{i}$ à l'étape actuelle mais calculée au niveau 1

$\left|c_{i}\right|=$ le nouveau degré de pertinence de l'atome de contexte i à l'étape actuelle 
De la même façon, pour chacune des trois (3) autres bases, il sera possible de régler les impasses en utilisant des fonctions identiques pour calculer les nouveaux degrés de pertinence:

- pour les éléments de la base Activité à l'aide de la fonction

$$
\begin{aligned}
& \left|a_{i}\right|=U_{i}(P, C, A) \\
& \left|a_{i}\right|=\frac{1}{n m} \sum_{k=1 \mid=1}^{n} \sum_{\sum}^{m} \frac{\sqrt{\left|a_{i} p_{k}\right|^{2}+\left|a_{i} c_{1}\right|^{2}+\left|a_{i}\right|^{2}}}{\sqrt{3}}
\end{aligned}
$$

- pour les éléments de la base Résultat à l'aide de la fonction

$$
\begin{aligned}
& \left|r_{i}\right|=V_{i}(C, A, R) \\
& \left|r_{i}\right|=\frac{1}{n m} \sum_{k=1 \mid=1}^{n} \frac{\sqrt{\left|r_{i} C_{k}\right|^{2}+\left|r_{i} a_{1}\right|^{2}+\left|r_{i}\right|^{2}}}{\sqrt{3}}
\end{aligned}
$$

- pour les éléments de la base Programme à l'aide de la fonction

$$
\begin{aligned}
& \left|p_{i}\right|=X_{i}(A, R, P) \\
& \left|p_{i}\right|=\frac{1}{n m} \sum_{k=1}^{n} \sum_{=1}^{m} \frac{\sqrt{\left|p_{i} a_{k}\right|^{2}+\left|p_{i} n_{1}\right|^{2}+\left|p_{i}\right|^{2}}}{\sqrt{3}}
\end{aligned}
$$


Au niveau 2, le système recalcule de nouveaux degrés de pertinence pour chacun des atomes d'une base de faits. A ce moment, deux possibilités se présentent au système:
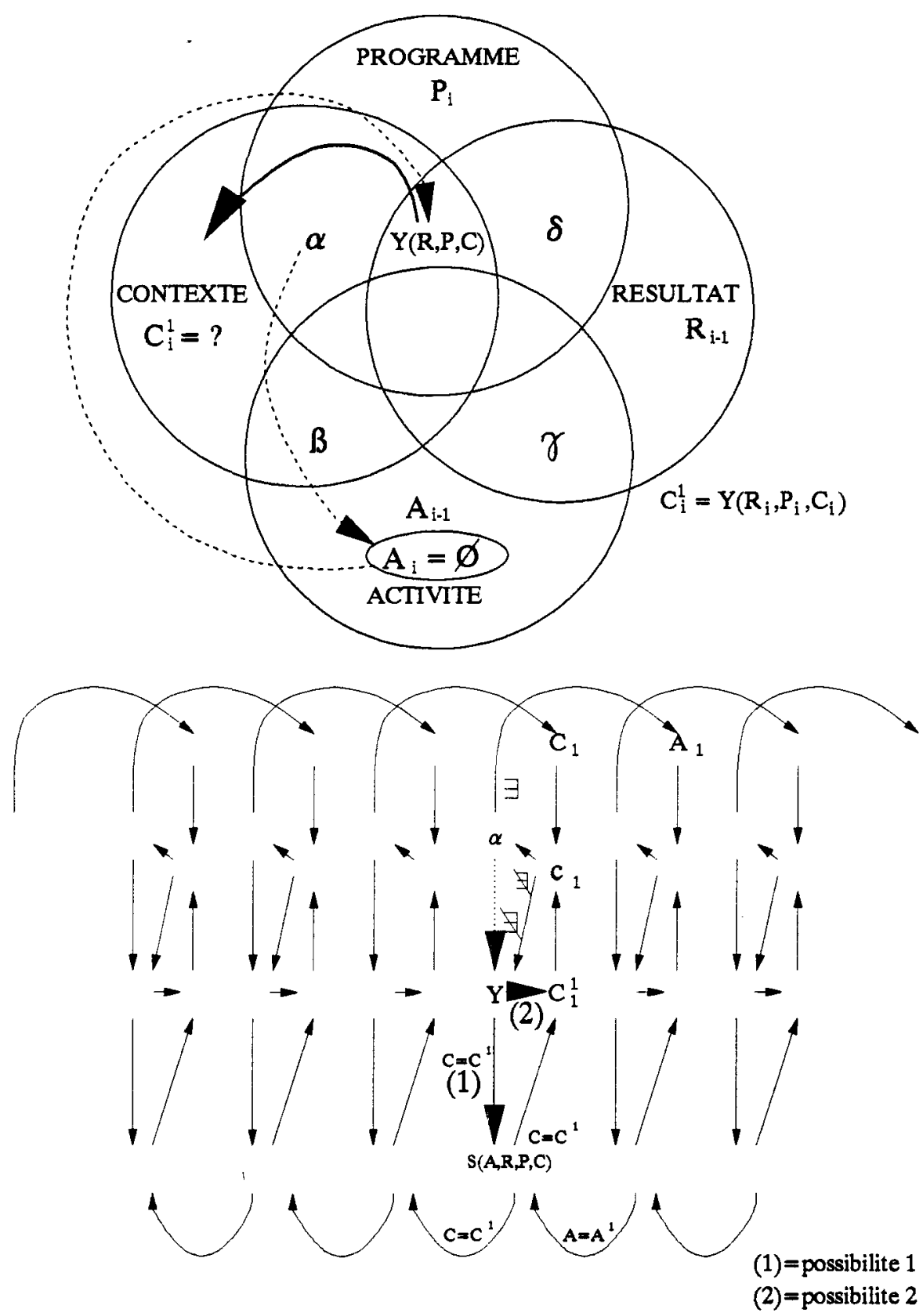

FIGURE 4.12: La trace lors du passage au niveau 2 


\section{Possibilité 1:}

II se peut qu'on retrouve, avec ces calculs, les mêmes degrés de pertinences pour chacun des éléments de contexte. II sera alors inutile de retourner au niveau 1 pour trouver une nouvelle activité compatible puisqu'on trouvera, de toute évidence, exactement la même chose qu'auparavant soit aucune activité compatible. La solution est alors d'aller au niveau 3, celui du superviseur qui fera possiblement appel à un expert humain.

\section{Possibilité 2:}

II se peut qu'on ait réussit à modifier les degrés de pertinence des éléments de la base. II est alors possible de faire un nouveau choix d'élément le plus pertinent dans ce nouvel ensemble de faits compatibles. A l'aide de ce nouveau fait local (l'élément le plus pertinent), le système retourne au niveau 1 afin d'essayer à nouveau de trouver un fait pertinent dans la base où il y avait impasse.

Dans tous les cas, le niveau 2 permet de lever l'impasse puisque, s'il demeure impossible de retourner au niveau 1, le problème sera acheminé au superviseur (niveau 3).

\section{8: Niveau $(-1)$}

Ce niveau de règles rend le système rétroactif en lui permettant de modifier certaines normes calculées par le système selon des évaluations externes. Au niveau 0 , lorsque le système demande la note de l'apprenant pour l'activité qu'il vient de faire, le système utilise le résultat de l'élève pour corriger le degré de pertinence de l'activité concernée.

II est sans conteste que cette note nous informe directement sur le résultat lié à cette activité mais elle peut également nous servir de guide concernant les 
autres bases. En effet, étant donné que le choix de l'activité repose sur les choix de contexte et de programme, la note obtenue permet de reconsidérer ces choix. En se servant du résultat obtenu à l'activité pour reconsidérer notre choix de contexte, une rétroaction sur une base est provoquée d'où l'appellation "fonction de niveau $(-1)^{\prime \prime}$.

\subsection{Niveau (-2)}

Pour les mêmes raisons qui justifient le niveau $(-1)$, on peut créer un niveau $(-2)$ de règles. Ce niveau fonctionne de la même façon que le précédent. II suffit de se servir de la note obtenue à l'activité par exemple pour modifier les normes liées aux éléments de programmes. Ce fait entraîne une rétroaction sur deux bases; on obtient donc une règle de niveau (-2).

\subsection{0: Conclusion}

Nous avons fait, dans le présent chapitre, une hypothèse de fonctions pour chacun des niveaux de règles possibles; plusieurs autres approches sont également possibles. Nous avons également limité le nombre de règles en simplifiant le modèle proposé parce que nous ne désirions qu'en démontrer la faisabilité. Cependant, ayant trouvé différentes fonctions permettant une gestion efficace du système préconisé, nous devons nous doter d'une définition plus spécifique des bases de connaissances à utiliser ainsi que de leur structure. C'est à travers ces bases que l'apprentissage ou tout autre problème sera géré. Le chapitre suivant montrera des structures possibles pour chacune des bases de notre système. Ces structures permettront une gestion utilisant les règles que nous venons de définir. 


\section{CHAPITRE V}

STRUCTURATION DES BASES DE CONNAISSANCES 


\section{CHAPITRE V}

\section{STRUCTURATION DES BASES DE CONNAISSANCES}

\section{0: Introduction}

Comme nous l'avons vu dans les chapitres précédents, le système considéré ici comporte quatre bases de connaissances:

- la base PROGRAMME contenant les éléments de programme à maîtriser par l'apprenant,

- la base CONTEXTE contenant les composantes psychologiques de l'apprenant,

- la base ACTIVITE contenant les diverses activités pédagogiques pouvant être offertes à l'apprenant pour parfaire son apprentissage,

- la base RESULTAT contenant les différentes évaluations du processus d'apprentissage.

Chacune de ces bases possède sa propre structure interne qui n'est toutefois pas restrictive. Nous définirons, dans le présent chapitre, chacune des bases utilisées de façon spécifique. 


\section{1: Base Programme}

La base PROGRAMME contient les différents sujets que l'étudiant doit maîtriser. Il s'agit en fait d'un certain nombre d'objets de connaissances ou d'éléments de programmes ( $\mathrm{Pii}$ ) structurés et formant les noeuds d'un réseau. Chaque noeud représente un élément de programme avec ses propres objectifs en même temps qu'un ensemble comprenant d'autres éléments du programme qui découlent de lui. Nous pouvons représenter schématiquement cette base sous la forme suivante:

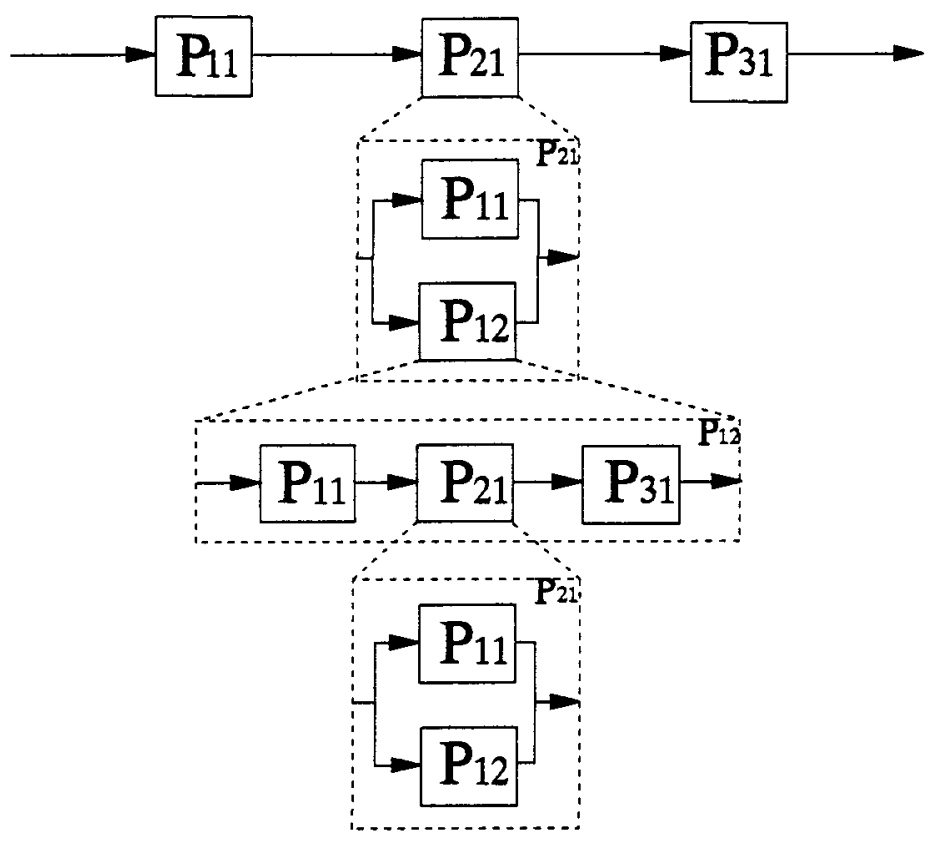

Pij est un sous-ensemble d'objets de connaissances structurées en un réseau de dépendance avec:

$P i j=(P 1 j, P 2 j, \ldots, P n j)$ si les objets sont placés en série

$\mathrm{Pij}=(\mathrm{Pi1}, \mathrm{Pi} 2, \ldots, \mathrm{Pim})$ si les objets sont placés en parallèle. 
Cette structure permet à l'apprenant de se trouver à un niveau de programme qui lui est adapté. Si l'étudiant réussi les objectifs de ce sujet, il est invité à poursuivre avec un autre élément du même niveau. S'il échoue, alors sa situation change et il passe à un niveau inférieur où les éléments de programmes sont plus raffinés. C'est de cette manière que la structure atomique de la base PROGRAMME nous permet d'aller se positionner au niveau cognitif de l'apprenant.

La structure de cette base permet au système d'aller se positionner en tout temps sur un concept pertinent pour l'apprenant en provoquant "l'explosion" des objets de connaissances en différents sous-objets lorsque c'est nécessaire. Cette explosion des éléments de programme vise l'obtention d'unités de plus en plus fines permettant de réduire le contenu du cours à des sujets élémentaires (Depover [11]).

\section{2: Base Contexte}

Cette base sert à tenir compte du vécu de l'apprenant dans le but de rendre cohérent le concept à apprendre (Lindsay et Norman [31]. Elle trouve son utilité lors de la mise en place d'organisateurs (ou préparateurs) d'apprentissage représentant un ensemble complexe d'idées ou de concepts présentés à l'étudiant avant que ce qu'il doit apprendre lui soit présenté. Cet organisateur dont traite Ausubel [54] sera d'autant plus efficace qu'il tiendra compte de l'individualité de l'apprenant. C'est de là que la base CONTEXTE tire toute son importance puisque la façon de présenter les notions et le contenu de l'organisateur peut différer selon le type de l'apprenant. La base CONTEXTE doit donc contenir toute information pertinente concernant l'apprenant; on y trouvera ainsi des informations au sujet du type d'usager à qui on a affaire. 
Cette information semble assez difficile à évaluer car elle relève de la psychologie, toutefois il sera possible de tenir compte de certaines variables affectives telles:

- l'anxiété,

- la dimension (introversion, extraversion),

- le niveau de motivation de l'élève,

- l'intérêt de l'élève, etc.

Certaines variables sont également liées au temps comme la manipulation des temps de latence [23] et d'autres variables sont liées à la Programmation Neuro Linguistique (PNL) qui est très efficace pour fournir des outils d'évaluation et de stratégies.

Cette programmation neuro linguistique s'avère être un premier outil de détection du type de l'apprenant puisqu'elle permet de classer les individus en quatre catégories bien identifiées: visuel, auditif, kinesthésique et digital.

La manipulation des temps de latence permet de tenir compte des périodes d'inaction de l'apprenant, on peut ainsi penser à modifier la présentation d'une leçon selon le temps écoulé depuis la dernière leçon reçue sur le même sujet.

Les paramètres de la base CONTEXTE peuvent être regroupés sous la forme d'un tableau d'états multi-dimensionnel. Si on décide de considérer les niveaux PNL, les vitesses d'apprentissage et les temps de latence de l'apprenant, il prend la forme générale suivante. 


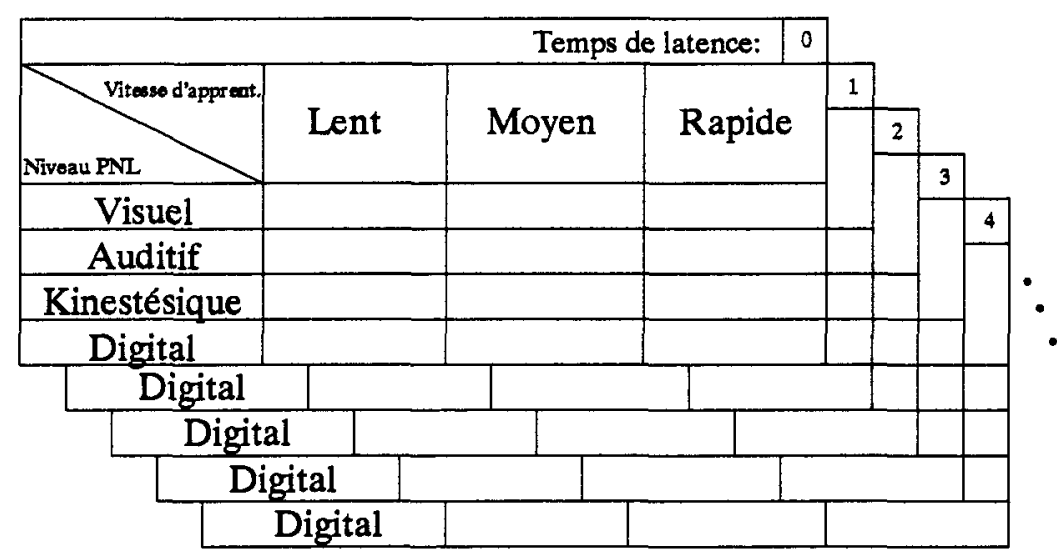

$C=(C 11 . .1, C 11 . .2, \ldots, C a b . . n)$

$\mathrm{Cij} . \mathrm{z}=$ niveau de contexte effectif

FIGURE 5.2 : Structuration de la base Contexte

Le contexte doit être initialisé à l'arrivée d'un nouvel usager à l'aide d'une interface contexte-extérieur. Ceci sera possible grâce à l'écoute pratique ou encore à un questionnaire de quelques questions seulement préparé et évalué par un spécialiste.

Cette première évaluation n'est pas finale mais est corrigée continuellement par le système grâce à l'expertise mise en place et à l'adaptabilité du modèle. Le contexte servira ensuite à faire un choix d'activités compatibles avec le caractère de l'usager ou encore à aborder une même activité de manière différente selon le caractère de l'apprenant. 


\section{3: Base Activité}

La base ACTIVITE contient un grand nombre d'activités d'apprentissage parmi lesquelles le système fera un choix tout au long du processus. Ces activités y sont représentées par des $n$-tuplets (vecteurs à $n$ dimensions ou ayant $n$ composantes). Les composantes de ces vecteurs représentent les paramètres nécessaires à l'identification et à la définition des activités. Chaque noeud représente donc une activité ou famille d'activités caractérisées par les noeuds qui le précèdent.

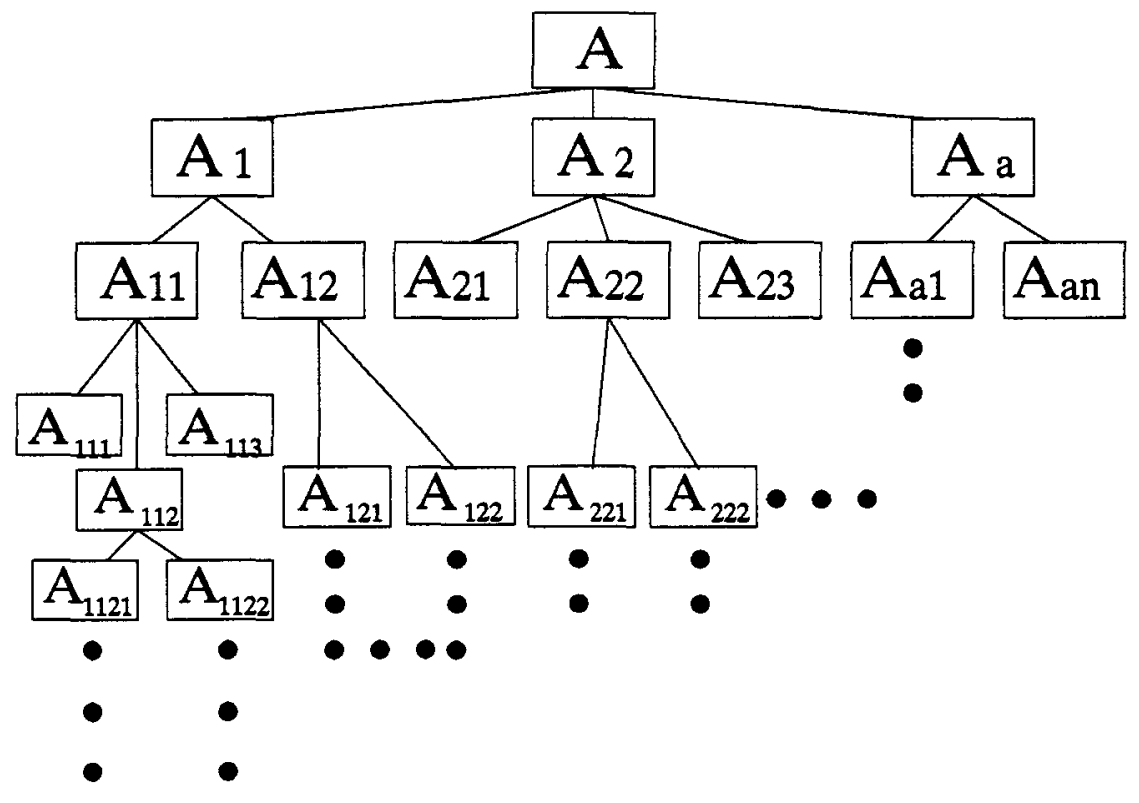

FIGURE 5.3 : Structuration de la base Actlvité

Au niveau de cette base se greffe obligatoirement une interface avec l'extérieur puisque c'est là que l'apprenant réagit à l'activité proposée. Cette base propose en effet une action à l'usager en provoquant l'interaction entre l'usager et l'activité en cours. Cette action de l'usager à l'instant $i$ entraîne un changement des normes dans les autres bases (contexte, programme et résultats). Ces 
changements permettent de définir l'activité qui suivra en modifiant les normes des différentes activités possibles.

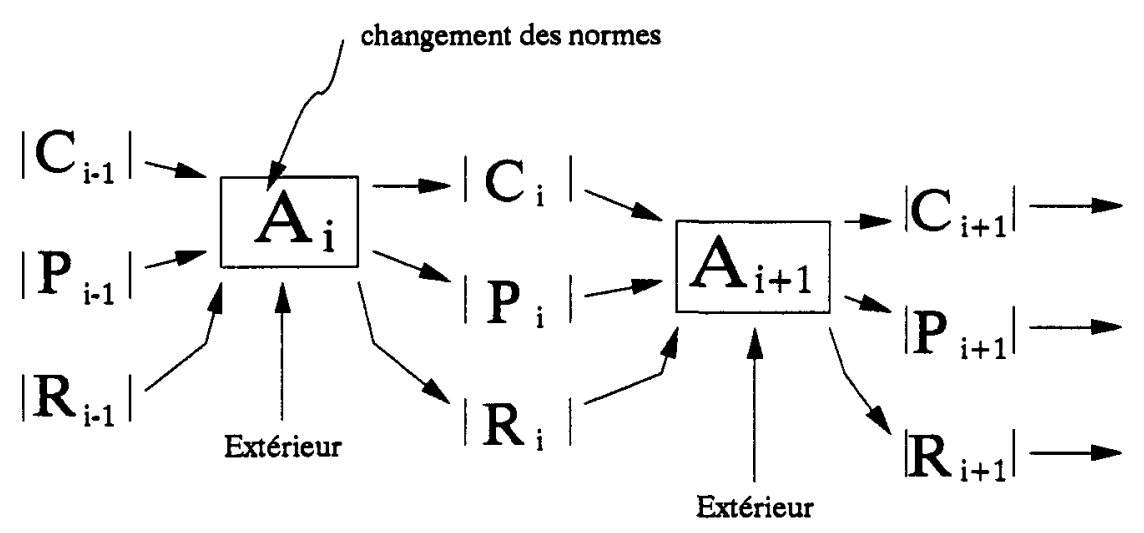

FIGURE 5.4 : Schéma fonctionnel du système autour de la base Activité

Ce schéma montre le degré minimal d'interaction du système. En fait, dans une situation idéale, il y interaction de chacune des bases avec l'extérieur via des interfaces questionnant l'usager lui-même ou un expert. Toutefois, le système sera fonctionnel même si son seul contact avec l'extérieur se fait au niveau de la base Activité. 


\section{4: Base Résultat}

La base RESULTAT contient les composantes de l'objet de contrôle (résultat) qui ne doit pas être seulement l'évaluation de l'élève mais aussi celle du cours lui même (Depover [11]). Cette évaluation permet, en plus de prendre une décision concernant l'apprenant, de rassembler des données qui servent de point de départ à une démarche d'amélioration de l'action de formation.

Pour Gagné [17], l'enseignant est un évaluateur et l'enseignant efficace se préoccupe de ce que chaque étudiant a appris en plus de décider de la façon d'arriver à des conclusions valides concernant les produits de l'apprentissage. D'ailleurs une formulation adéquate de l'interaction entre un organisme et son milieu doit toujours comporter trois choses (Skinner [47]):

-les circonstances dans lesquelles la réponse survient,

-la réponse elle-même, et

-les conséquences renforçantes.

C'est pour ces raisons que la base RESULTAT contient une évaluation non seulement sommative mais aussi contextuelle, normative et formative (chapitre 1). L'évaluation est représentée sous forme d'un n-tuplet d'objectifs hiérarchisés et exprimés en termes de verbes d'action.

Ri $=(\mathbf{R 1}, \mathbf{R 2}, \mathbf{R 3}, \ldots, \mathbf{R n})$

Ri est un vecteur de résultats concernant l'usager $i$

Ri est un vecteur de la forme

(Reconnaître l'objet parmi d'autres objets, Reconnaître l'objet dans une situation donnée, Utiliser l'objet lorsque demandé, Reconnaître le moment où on doit utiliser l'objet, Analyser un problème d'utilisation de l'objet, ...)

FIGURE 5.5 : Structuration de la base Résultat 
Le système possédant des couches supérieures de gestion peut évaluer un taux de réussite de ces différents objectifs tout en lui permettant de sophistiquer l'analyse de cette réussite. II est également possible d'utiliser la théorie de la formation de concepts de Woodruff [53] qui fournit une évaluation à la fois sommative et contextuelle afin mieux situer l'apprenant.

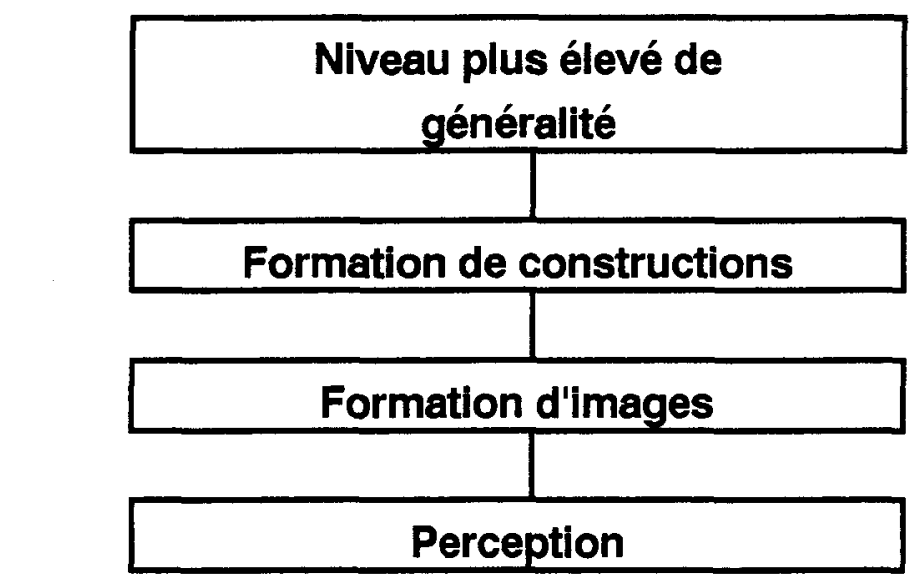

FIGURE 5.6: Figure hlérarchique de la formation d'un concept (Woodruff [53] p.16)

\section{5: Conclusion}

Nous avons fait, dans le présent chapitre, une proposition pour les contenus des différentes bases nécessaires au bon fonctionnement du système préconisé. Les structures des bases présentées sont des structures souhaitées mais il serait possible d'en définir d'autres pouvant servir tout aussi bien le système. II importe cependant de se souvenir que chacune des bases forme en soit un système expert même s'il n'est pas développé ici. Comme il s'agit d'un système gestionnaire d'une situation d'apprentissage, on est en présence d'un système de gestion d'un réseau de systèmes experts. 
CHAPITRE VI

\section{LES EXEMPLES}




\section{CHAPITRE VI}

\section{LES EXEMPLES}

\section{0: Introduction}

Dans le but de concrétiser le modèle proposé, il a été résolu de présenter deux exemples dont l'un a été programmé sur micro-ordinateur.

Un premier exemple gérant une situation d'apprentissage de l'algèbre booléenne de base a été choisi pour sa complexité puisqu'il représente une interaction humain-machine, une des situations les plus difficiles à gérer. L'étude de ce cas a conduit à la naissance du programme PROLIN: un gestionnaire d'apprentissage visant la simplification d'une expression booléenne donnée.

Le second exemple, plus simple, démontre la généralisation du modèle. II s'agit de la gestion d'un bras manipulateur (robot) représentant une interaction machine-machine.

Ces deux exemples seront complètement définis à travers le modèle proposé démontrant ainsi l'applicabilité et l'efficacité du modèle. 


\section{1: L'apprentissage de l'algèbre de Boole (programme PROLIN).}

Dans le but d'expérimenter et de bien comprendre les composantes et stratégies du modèle présenté, il a fallu choisir un exemple simple d'application pouvant être programmé sur micro-ordinateur. Parmi le très vaste choix qui se présentait à nous, nous avons opté pour l'algèbre booléenne et, plus précisément, pour la simplification d'une expression simple.

L'expression ainsi choisie est: $X Y \bar{Z}+X Y Z+Z(X+Y) \overline{(X \bar{Y})})$

\subsection{1: Théorèmes de base de l'algèbre booléenne}

Les théorèmes sont groupés par degré de difficulté. Ainsi, on trouve, au degré 1: les deux théorèmes d'éléments neutres et de complémentation

au degré 2: les deux théorèmes concernant la distributivité au degré 3: les deux lois de De Morgan.

Notons que les autres théorèmes (ceux de la commutativité et de l'associativité) ne sont pas incorporés dans le système parce qu'ils ne sont pas nécessaires pour résoudre l'expression donnée. Ils peuvent toutefois facilement s'ajouter à la base de connaissances si le besoin s'en fait sentir. Vous trouverez, dans l'encadré qui suit, le résumé de la théorie liée à l'algèbre de Boole pour laquelle les éléments non retenus dans l'exemple sont placés entre crochets. 


\section{Résumé de la théorie de l'algèbre de Boole.}

Postulats:

$$
\begin{array}{ll}
0+0=0 & 0 \cdot 0=0 \\
0+1=1 & 0 \cdot 1=0 \\
1+0=1 & 1 \cdot 0=0 \\
1+1=1 & 1 \cdot 1=1
\end{array}
$$

Théorèmes de degré $1:$

Elément neutre du ou:

Quelque soit $A ; 0+A=A+0=A$

Elément neutre du et:

Quelque soit $A ; 1 \cdot A=A \cdot 1=A$

Complémentation du ou:

Quelque soit $A ; A+\bar{A}=\bar{A}+A=1$

Complémentation du et:

Quelque soit $A ; A \cdot \bar{A}=\bar{A} \cdot A=0$

[ Commutativité du ou:

Quelques soient $A, B ; A+B=B+A$ ]

[ Commutativité du et:

Quelques soient A, B; A B $=B \cdot A$ ]

[Associativité du ou:

Quelques soient $A, B, C$;

$$
(A+B)+C=A+(B+C)]
$$

[Associativité du et:

Quelques soient $A, B, C$;

Théorèmes de degré 2 :

$$
(A \cdot B) \cdot C=A \cdot(B \cdot C)]
$$

Distributivité du et sur le ou:

Quelques soient $A, B, C$;

$$
A \cdot(B+C)=(A \cdot B)+(A \cdot C)
$$

Distributivité du ou sur le et:

Quelques soient $A, B, C$;

$$
A+(B \cdot C)=(A+B) \cdot(A+C)
$$

Théorèmes de degré 3 :

Première loi de De Morgan:

Quelques soient $A, B ; \overline{A+B}=\bar{A} \cdot \bar{B}$

Deuxième loi de De Morgan:

Quelques soient A, B; $\overline{A \cdot B}=\bar{A}+\bar{B}$ 


\subsection{2: Les bases de connaissances}

Voici maintenant les contenus des quatres bases de connaissances nécessaires au développement du système permettant la gestion de cette situation d'apprentissage.

\subsubsection{1: Base Programme:}

Cette base contient les différents sujets que l'étudiant doit maîtriser. Le schéma suivant représente cette base qui est formée de sujets élémentaires de la théorie de l'algèbre de Boole.

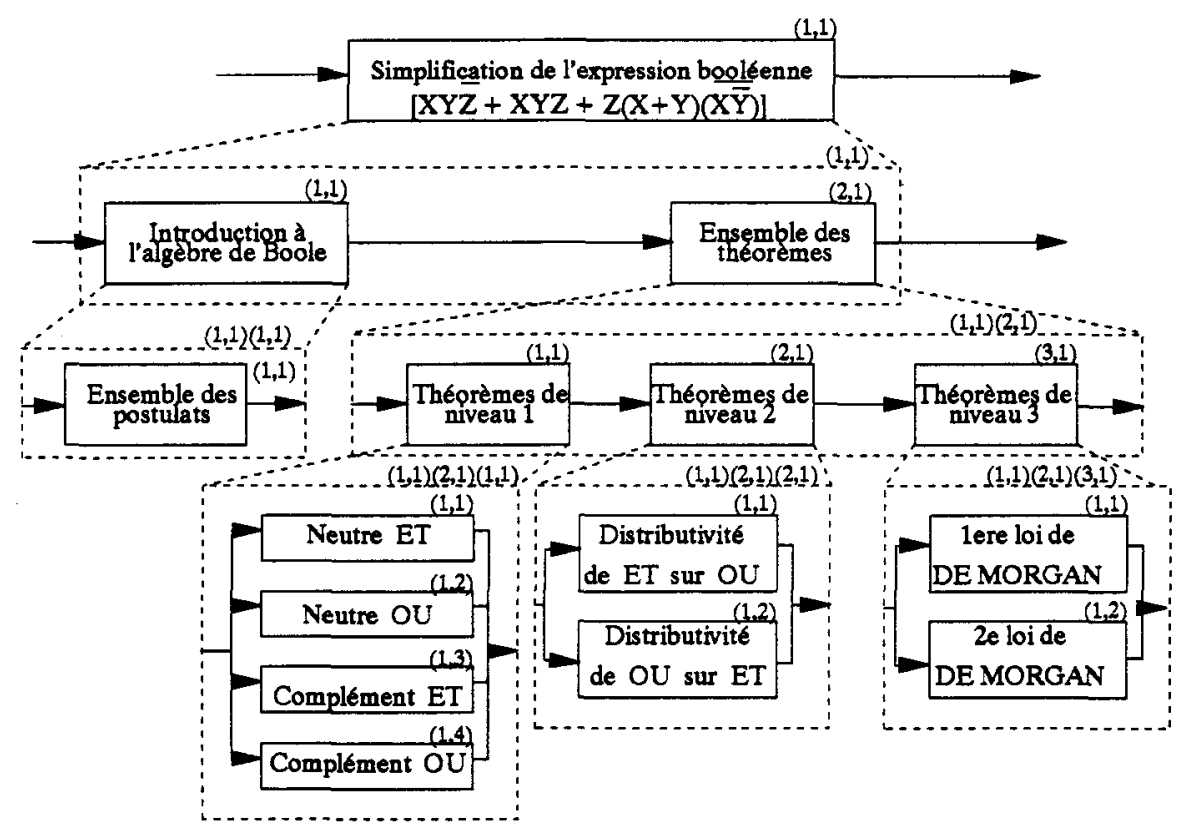

FIGURE 6.1 : Programme (Algèbre de Boole) 


\subsubsection{2: Base Contexte:}

Concernant notre exemple, la base CONTEXTE a été réduite à sa plus simple expression. Le système ne tient compte que du mode de communication privilégié de l'apprenant défini par la PNL (programmation neuro linguistique, chapitre 5) soit: visuel, auditif et kinesthésique et de la vitesse d'apprentissage de ce dernier soit: lent, moyen ou rapide.

\begin{tabular}{|c|l|l|l|}
\hline Niveau PNL & Lent & Moyen & Rapide \\
\hline Visuel & & & \\
\hline Auditif & & & \\
\hline Kinestésique & & & \\
\hline
\end{tabular}

FIGURE 6.2 : Contexte (Algèbre de Boole)

A l'intérieur de ce tableau, nous trouvons les degrés de pertinence associés à chacune des situations possibles. Nous avons donc un tableau comprenant 9 paramètres qui sont modifiés à chaque étape du processus d'apprentissage et qui serviront à choisir, parmi les types présents, celui qui caractérise le mieux l'apprenant. 


\subsubsection{3: Base Activité:}

La figure suivante montre la structure de la base ACTIVITE liée à notre exemple d'application d'enseignement de l'algèbre de Boole. Remarquons que cette base, comme les autres, n'est aucunement fermée et que les ajouts seront les bienvenus en temps opportun.

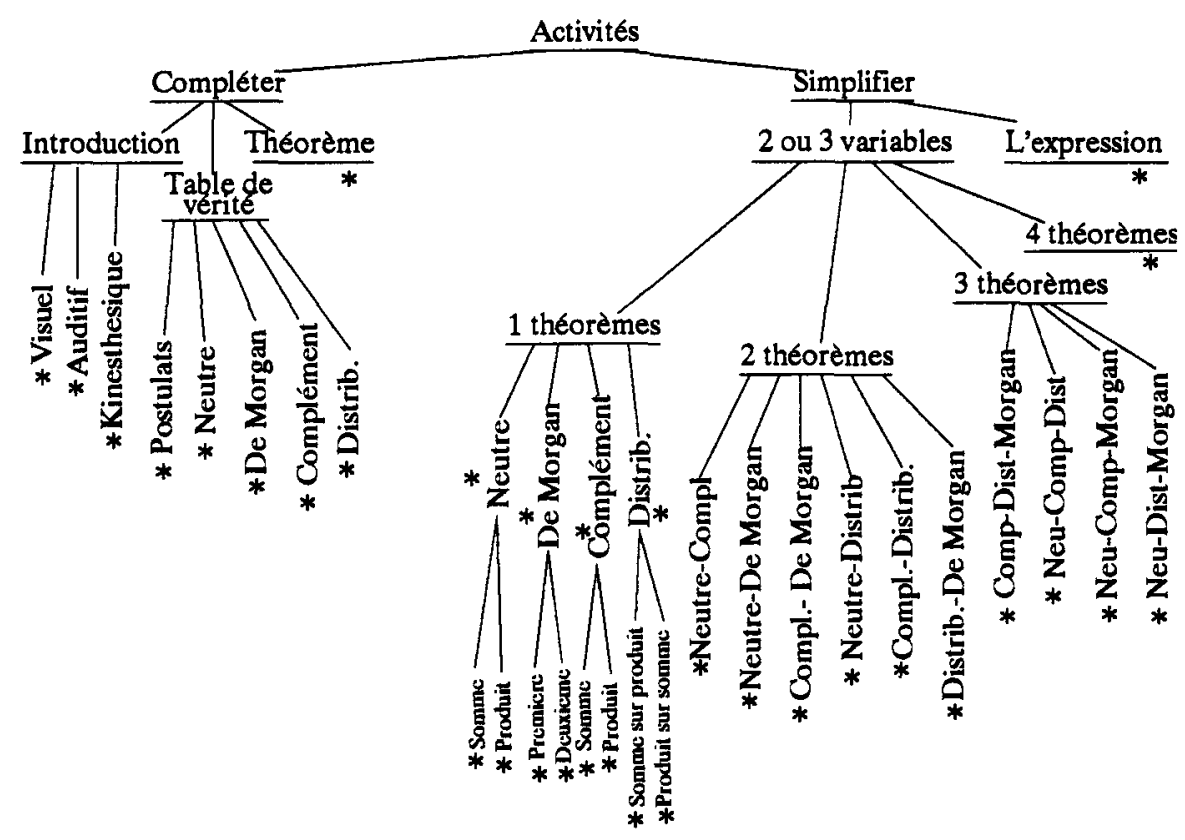

FIGURE 6.3 : Activité (Algèbre de Boole)

Les activités sont regroupées dans un cahier pour son utilisation par l'usager de PROLIN et disponibles dans la partie 4 du guide technique. Remarquons que dans le logiciel PROLIN, il y a une activité correspondant à chaque noeud marqué d'un astérisque (*), il y a donc 33 activités. 


\subsubsection{4: Base Résultat:}

La base résultat de l'exemple qui nous intéresse se résume à la suivante, même si elle pourrait, en théorie, être beaucoup plus complexe.

R1: Utiliser les théorèmes d'éléments neutres

R2: Utiliser les théorèmes de complémentation

R3: Utiliser les théorèmes de distributivité

R4: Utiliser les lois de De Morgan

R5: Simplifier une expression à deux variables

R6: Simplifier une expression à trois variables

R7: Simplifier l'expression donnée

R8: Utiliser les postulats de l'algèbre booléenne

FIGURE 6.4 : Résultats (Algèbre de Boole)

PROLIN considère l'objectif global atteint lorsque le résultat $\mathrm{R7}$ prend la valeur 1 , c'est-à-dire lorsque l'apprenant a réussi à simplifier l'expression donnée. 


\subsection{3: Programme PROLIN}

Ces bases dont il est question plus tôt ont été implantées dans le programme PROLIN pour lequel vous trouverez plus de détails dans les chapitres ultérieurs.

Remarquons que lors de la programmation de cet exemple, la présence d'experts manquant, les normes de compatibilité deux à deux ont été définies selon les humbles connaissances de l'auteure. Elles ne sont donc pas parfaites et on peut les soupçonner responsables de certains problèmes mineurs du système. En effet, il est possible que le système fasse un mauvais choix dans l'une ou l'autre des bases de faits à cause de ces informations biaisées. Toutefois, la démonstration d'applicabilité du modèle ne souffrira pas, nous l'espérons, de cette lacune qui sera d'ailleurs facilement corrigée si on y met le temps et les experts nécessaires.

Nous venons de voir une utilisation possible du modèle préconisé; il en existe plusieurs autres. En fait, tout ce qui représente une résolution de problème au sens large peut trouver avantage à être gérer à l'intérieur de ce modèle. Vous trouverez à la section suivante la présentation d'un deuxième exemple (robot) démontrant une autre utilisation possible du modèle. 


\section{2: La gestion d'un bras manipulateur}

La présentation de ce deuxième exemple a pour but de démontrer l'adaptabilité" du modèle. Depuis le début, il est mentionné que ce dernier représente un modèle général et applicable dans à peu près tous les domaines. C'est donc pour appuyer cette affirmation qu'un deuxième exemple, dans un domaine tout à fait différent, est présenté ici. Cet exemple se situe même "à l'opposé" du premier puisque la gestion d'un bras manipulateur exclue totalement les rapports humains alors que dans le premier ils sont omniprésents. Dans la plupart des cas de gestion de projets quelconques, on se situe quelque part entre ces deux extrêmes.

\subsection{1: Présentation de l'exemple}

Le but du problème qui nous occupe est de guider un bras manipulateur afin qu'il puisse placer un manche trop lourd pour la manipulation humaine au fond d'un trou serré [19]. Si ce manche était de petite dimension, ça représenterait un travail très simple pour un humain. Ce n'est toutefois pas aussi facile pour un robot qui est incapable de porter à la fois un jugement local et global puis prendre une décision par rapport à cette évaluation de la situation. Dans ce qui suit, nous présenterons le contenu des bases nécessaires à ce type de gestion. Notons toutefois que cet exemple n'a pas été programmé. 


\subsection{2: Les bases de connaissances}

\subsubsection{1: Base Programme:}

La base PROGRAMME du système visant la gestion d'un bras manipulateur qui insère un manche à l'intérieur d'un trou serré se structure de la même façon qu'un programme d'enseignement. II sera ainsi permis de raffiner autant que nécessaire les mouvements du robot. Le schéma qui suit montre à quoi peut ressembler le contenu de la base PROGRAMME d'un tel robot.

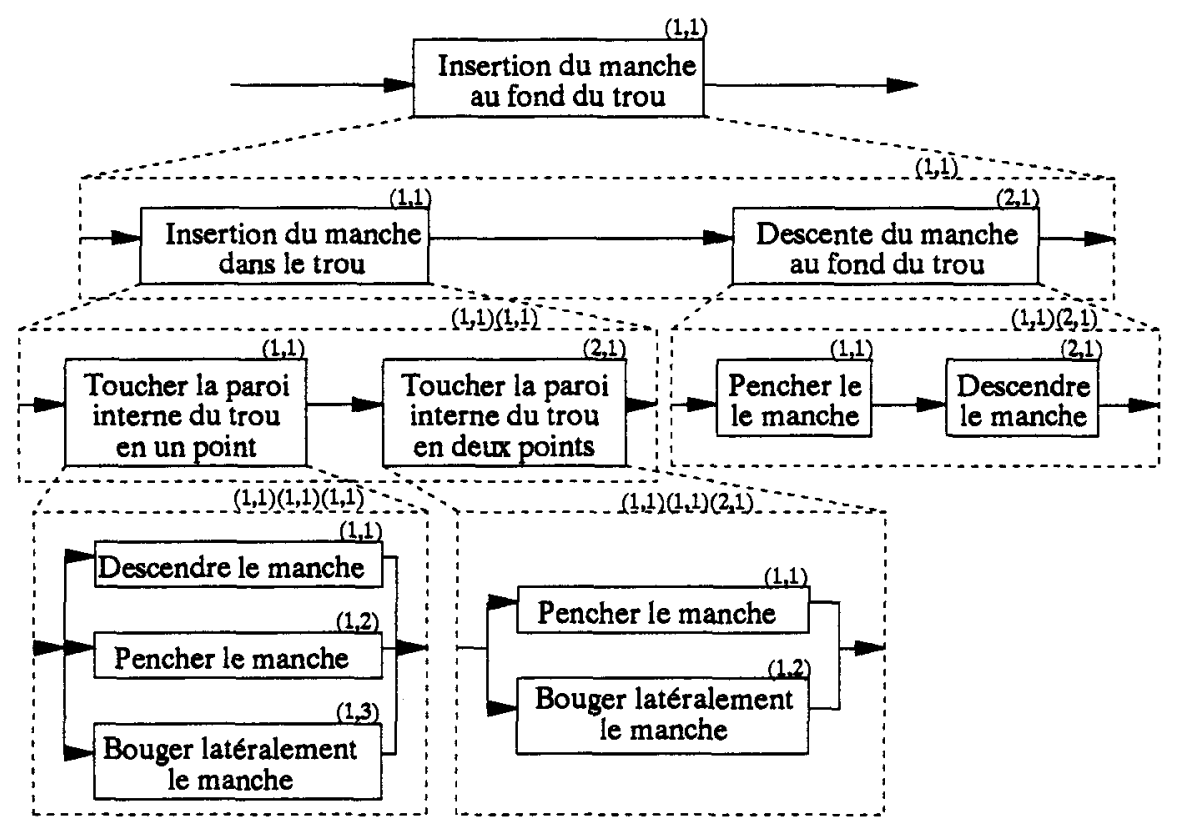

FIGURE 6.5 : Programme (Bras manipulateur)

\subsubsection{2: Base Contexte:}

Concernant le bras manipulateur, la base CONTEXTE se trouve dégénérée à moins que certains mouvements soient physiquement restreints par un espace réduit ou par d'autres limites techniques. Donc, de façon générale, la gestion d'un 
robot comme le bras manipulateur se fait sans problème à l'intérieur d'un système à trois bases de connaissances (Programme-Activité-Résultats).

\subsubsection{3: Base Activité:}

Ce qui nous intéresse ici, c'est de faire bouger le manche. Certains mouvements permettent de le placer à l'endroit désiré, les activités du système se résument donc à ces mouvements. II importe également de définir l'amplitude de ces mouvements et c'est à ce niveau que l'intelligence artificielle peut aider le robot à acquérir un comportement plus "intelligent". La base ACTIVITÉ pour notre bras manipulateur ressemble donc à la suivante.

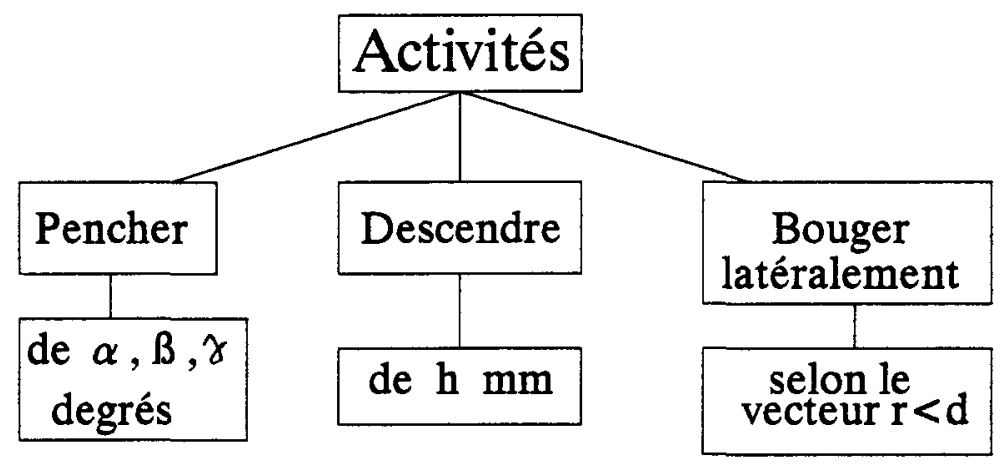

FIGURE 6.6 : Activité (Bras manlpulateur)

Effectivement, les mouvements du robot se résument à:

- pencher le manche de:

$\alpha$ degrés selon l'axe des $x$,

$\beta$ degrés selon l'axe des $y$, et 
$\gamma$ degrés selon l'axe des $\mathrm{z}$;

- descendre le manche de $h$ millimètres; ou

- bouger latéralement sur le plan se situant au dessus du trou selon un vecteur de déplacement $r$ de longueur inférieure à la distance $d$ séparant le manche du trou.

Une gestion efficace de l'ensemble de ces mouvements permettra d'atteindre l'objectif final du processus: placer le manche au fond du trou, avec un minimum d'efforts.

\subsubsection{4: Base Résultat:}

Pour notre bras manipulateur, on peut choisir comme base RÉSULTAT la suivante.

R1: le manche est a une distance $X$ du fond du trou

R2: le manche est à une distance $X$ du trou

R3: le manche est coincé avec deux points d'appui à l'intérieur du trou

R4: le manche est à une hauteur $\mathrm{H}$ au dessus du trou

FIGURE 6.7 : Résultats (Bras manlpulateur)

Nous pouvons considérer l'objectif final atteint lorsque dans $\mathrm{R} 1$, la variable $\mathrm{X}$ prend la valeur 0 .

La gestion du bras manipulateur n'a pas été programmée mais nous croyons que ce ne serait pas un gros problème. II ne suffirait, en effet, qu'à modifier le programme de l'exemple de Boole. Toutefois, comme seulement trois bases de 
connaissances suffisent à la gestion de ce bras manipulateur, deux options s'offrent à nous pour le gérer:

- utiliser le même modèle à quatre bases de connaissances en assignant une importance nulle à la base CONTEXTE, ou

- utiliser un modèle semblable mais qui n'utilise que trois bases de connaissances.

Ces deux approches sont présentées à l'annexe 2.

Cet exemple nous a permis de montrer la polyvalence du modèle proposé. II est donc permis de croire que la qualification de modèle général de gestion convient à celui que nous proposons.

\section{3: CONCLUSION}

Nous avons démontré l'applicabilité du modèle proposé à l'aide de deux exemples d'application. Plusieurs autres exemples auraient pu être considérés. Par exemple, on aurait pu baser l'approche sur une résolution de problème en gestion de projet, en finance, une partie d'échec, ou encore l'initiation à la théorie musicale qui représente un exemple très riche à tous les points de vue et possède l'avantage d'être plus polyvalent au niveau contextuel. II suffirait en effet de brancher un synthétiseur numérique à l'ordinateur pour faire entendre quelque chose à l'apprenant ou encore lui faire jouer un air puis corriger ses fautes de rythme ou de lecture. Cela demanderait cependant un plus grand effort technologique de démonstration et là n'est pas le but de la présente recherche.

Tout ce qui représente une résolution de problème au sens large peut être implanté au système. Cette caractéristique fait de ce dernier un système général. 


\section{CHAPITRE VII}

\section{LE LOGICIEL PROLIN}




\section{CHAPITRE VII}

\section{LE LOGICIEL PROLIN}

\section{0: Introduction}

Le but de la présente recherche étant de produire un système informatique de gestion générale de ressources, la production d'un logiciel est fondamental. La programmation d'un tel système demandant l'apport de petits trucs et astuces qu'il serait long et fastidieux d'expliquer, nous préférons présenter le logiciel à travers une analyse fonctionnelle globale qui permettra, nous l'espérons, de bien cerner ce qui a été fait sans toutefois risquer de se perdre dans d'inutiles détails.

Puisque notre travail visait l'obtention d'un logiciel "intelligent", l'utilisation de l'intelligence artificielle comme outil de programmation semblait aller de soit; c'est pourquoi nous avons choisi d'utiliser le langage PROLOG, et plus particulièrement ARITY-PROLOG qui, en plus de représenter un outil très performant et polyvalent, offre des possibilités intéressantes comme:

- la possibilité de programmer dans un interpréteur évitant d'avoir à compiler le programme trop souvent,

- la présence d'un "debugger" avec espions permettant de découvrir plus aisément les erreurs en cours de route.

Le logiciel développé dans la présente recherche porte le nom de PROLIN: PRO en raison du langage utilisé (PROLOG) et LIN pour Linda (auteure et conceptrice du programme informatique). 


\section{1: Analyse fonctionnelle du programme PROLIN}

Le schéma fonctionnel qui suit (figure 7.1) montre les principaux modules du programme PROLIN ainsi que les liens les unissant. II est facile d'y percevoir les niveaux 1,2 et 3 de règles:

$\begin{array}{lll}\text { niveau } 1 & -> & \text { module calcul norme } \\ \text { niveau } 2 & -> & \text { module niveau } 2 \\ \text { niveau } 3 & -> & \text { module niveau } 3 \text { ou du superviseur }\end{array}$

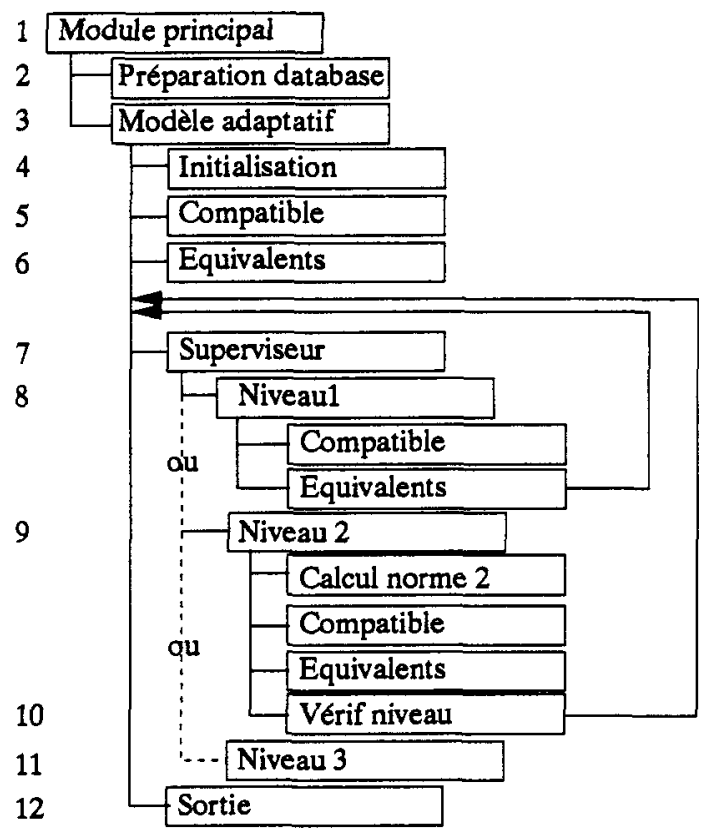

FIGURE 7.1 : Schéma fonctionnel du programme PROLIN

Remarque: les numéros inscrits à gauche correspondent aux numéros des sections concemant les différents modules mentionnés.

Les niveaux 0 et $(-1)$ sont présents quoique moins visibles. C'est pourquoi les sections 7.1.13 et 7.1.14 leurs sont réservées. Les autres sections concernent les modules visibles du schéma fonctionnel. Le lecteur trouvera dans la partie $1 \mathrm{du}$ rapport technique le listage complet du programme PROLIN pour lequel suivent quelques explications concernant les principaux modules. 


\subsection{1: Module principal}

Le rôle de ce module est de gérer le début des opérations en s'occupant des technicalités qui y sont associées. II s'agit en fait du premier module du programme. Il comprend les deux modules suivants:

- module de préparation de la base de connaissances (Préparation database),

- module de gestion générale du système (Modèle adaptatif).

\subsection{2: Module de préparation de la base de connaissances}

Ce module s'occupe de la création de la base de connaissances (ou de faits) et de son initialisation. Cette dernière se fait en transférant dans la base les données qui se trouvent dans des fichiers textes. PROLIN ne travaille par la suite qu'avec cette base de connaissances qu'il vient de créer.

Les fichiers textes où PROLIN va lire les données dont il a besoin sont les fichiers "PCAR" et "NORMES" décrits ci-dessous et présentés dans la partie 2 du rapport technique.

\section{FICHIER "PCAR"}

Le fichier "PCAR" contient tous les renseignements nécessaires concernant les quatres ensembles PROGRAMME, CONTEXTE, ACTIVITE et RESULTAT sous la forme:

pcar(programme,a,b,c,d,e,f,g)

pcar(contexte, a,b,c,d,e,f,g)

pcar(activité,a,b,c,d,e,f,g)

pcar(résultat,a,b,c,d,e,f,g)

où $\quad \mathrm{a}=$ le numéro de l'élément de la base concernée 
$b=$ la limite inférieure d'acceptation du degré de pertinence

$c=$ le degré de pertinence de l'élément concerné

$d=$ la limite supérieure d'acceptation du degré de pertinence

$e=l e$ degré de pertinence de l'élément concerné à l'étape précédente

$f$ et $g$ sont des valeurs de contrôle pour la recherche de l'élément le plus compatible

On y trouve de plus d'autres renseignements nécessaires à la gestion du système comme:

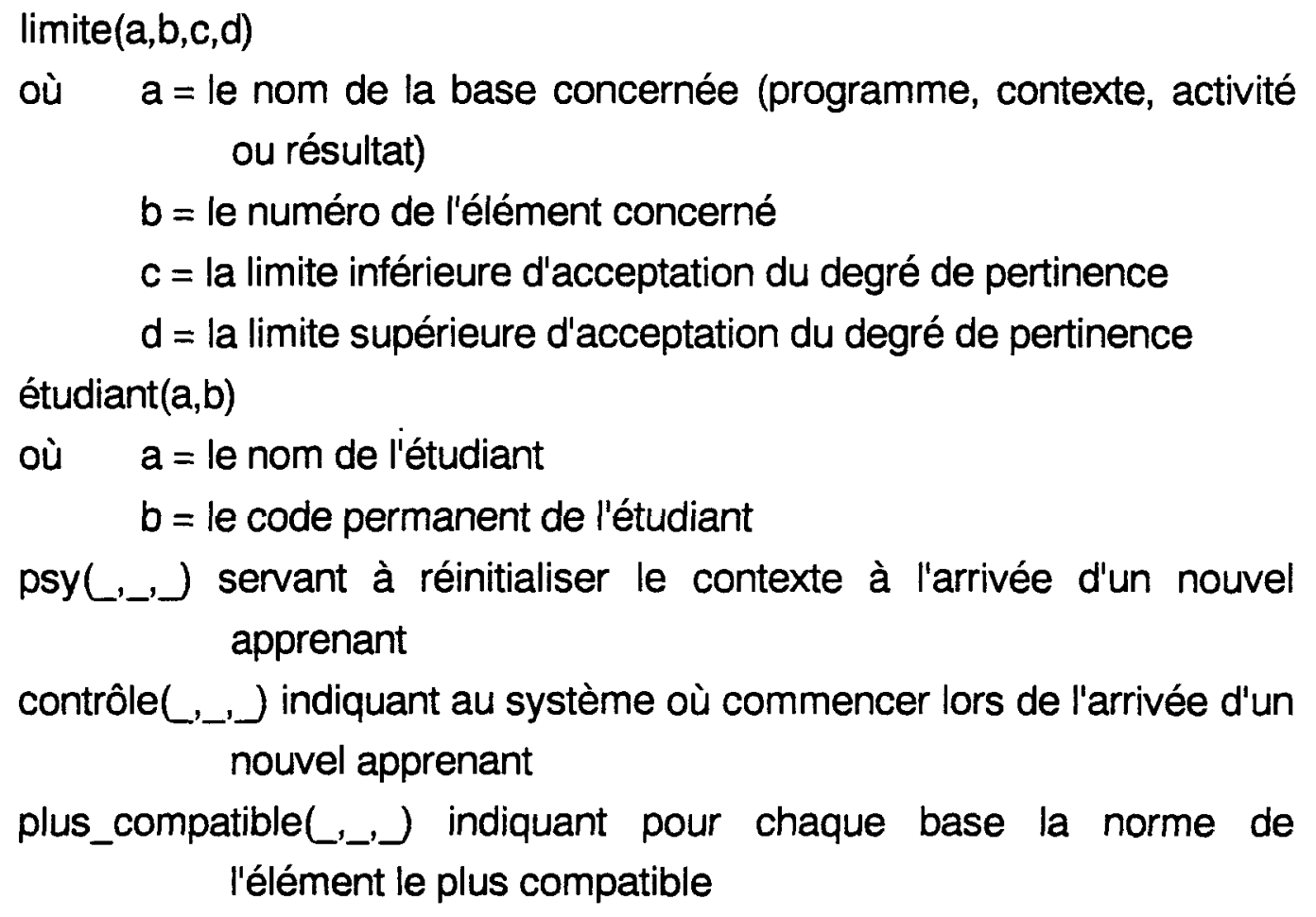
apprenant

contrôle (_,_. $)$ indiquant au système où commencer lors de l'arrivée d'un nouvel apprenant

plus_compatible (_,_) indiquant pour chaque base la norme de l'élément le plus compatible

\section{FICHIER "NORMES"}

Le fichier "NORMES" contient les normes de compatibilité des différents faits pris deux à deux. On y trouve les normes de compatibilité:

activité-programme

activité-contexte, résultat-contexte, résultat-activité, 


$$
\begin{aligned}
& \text { programme-activité, } \\
& \text { programme-résultat, } \\
& \text { contexte-résultat, } \\
& \text { contexte-programme }
\end{aligned}
$$

sous la forme:

norme(a,b)

où $a$ = aipj représentant l'activité i étant donné le programme j ou, de la même façon, aicj, ricj, riaj, piaj, pirj, cirj ou bien cipj $\mathrm{b}=$ la norme de compatibilité associée à la situation décrite en (a)

Remarquons que les contenus de ces fichiers "PCAR" et "NORMES" demeurerons toujours intacts; PROLIN s'y référera à chaque fois qu'il a besoin de réinitialiser le système.

\subsection{3: Module global (Modèle adaptatif)}

Ce module s'occupe des premières opérations à effectuer avant de pouvoir donner le contrôle au superviseur, donne le contrôle au superviseur qui gérera l'ensemble des opérations qui suivront, et gère la fin des opérations.

Ce module comprend les quatre modules suivants:

- module d'initialisation du système (Initialisation),

- module de recherche du plus grand fait compatible (Compatible),

- module du superviseur,

- module de gestion de la fin des opérations (Sortie).

Après l'initialisation, les calculs nécessaires sont réalisés pour trouver les éléments de contexte compatibles et le plus pertinent de ces éléments. De cette façon, un premier élément de contexte à considérer est trouvé et la prochaine étape 
consiste à choisir une activité à proposer à l'usager. Cette dernière peut s'exécuter normalement, c'est-à-dire en utilisant les règles de niveau 1 ou 2 selon le choix du superviseur.

\subsection{4: Module d'initialisation du système}

Le but de ce module est l'initialisation du système. Ce dernier vérifie dans un premier temps si l'usager est connu du système..Dans ce cas, l'étudiant reprend la leçon là où il l'avait laissée lors de son dernier passage. Si, par contre, il s'agit d'un nouvel usager, le module vérifie si la base est vide, c'est-à-dire non modifiée par rapport aux fichiers initiaux. Si ce n'est pas le cas, le module vide la base, la reconstruit à partir des fichiers, puis fait appel à la règle "init_psy(Nom,Code)" qui demande les valeurs des paramètres psychologiques de l'apprenant et trouve les premiers degrés de pertinence des faits de la base Contexte.

Notons bien qu'à cette étape, le système possède déjà un élément de programme compatible; il est défini par défaut dans le fichier "PCAR" (section 7.1.2). Le système possède de plus, grâce à ce module, des degrés de pertinence pour les éléments de la base Contexte, ce qui permet de déterminer l'élément de contexte le plus compatible. Nous sommes donc prêt à donner le contrôle au superviseur qui pourra faire un premier choix d'activité d'apprentissage avec les résultats fournis.

\subsection{5: Module de recherche du plus grand compatible}

Le module "plus grand compatible" a pour seul but de trouver la valeur du fait le plus compatible. Les faits sont dits compatibles lorsque leur valeur se situe dans un certain intervalle de compatibilité qui dépend de chacun des faits et est 
initialisé avant le début de l'expérience par un expert extérieur. On trouve ces valeurs dans le fichier "PCAR" à la rubrique "limite" (section 7.1.2).

La recherche du fait le plus compatible se fait par l'analyse de listes. On crée d'abord une liste de faits retenus (liste de tous les faits compatibles) et, de cette liste, on retient la valeur du ou des faits les plus compatibles. Si plusieurs faits peuvent être qualifiés de plus compatibles, c'est qu'ils ont la même valeur. Cette dernière est donc retenue dans la variable réponse d'où on la traitera par la suite.

Le choix du plus grand fait compatible se fait en considérant simplement la valeur des normes de compatibilité des faits retenus et en choisissant celui qui présente la plus grande norme. Cette manière de faire se justifie par la facilité d'utilisation d'un tel algorithme.

\subsection{6: Module de recherche des faits équivalents}

Ce module est nécessaire car il arrive que plus d'un fait puissent être considérés comme le plus grand fait compatible. II répond à la question du choix à faire lorsque plusieurs faits offrent la même compatibilité en amenant le système à choisir au hasard parmi tous les faits les plus compatibles. Ainsi, connaissant la valeur du ou des faits les plus compatibles, on recherche le numéro de référence d'un fait qui sera considéré par la suite comme le fait le plus pertinent.

\subsection{7: Module du superviseur}

Par le biais de ce module, le superviseur contrôle le système en entier. II envoie les règles adéquates à l'exécution pour rechercher le prochain fait compatible. II choisi ainsi entre les règles de niveau 1, de niveau 2, ou encore il 
peut provoquer la fin des opérations, en passant par le niveau 3, si cela est nécessaire.

Il comprend les modules:

- de gestion du niveau 1 de règles,

- de gestion du niveau 2 de règles, et

- de gestion du niveau 3 de règles.

Le superviseur fera un choix parmi ces trois modules à chaque étape du processus.

\subsection{8: Modules de calcul des normes (Niveau 1)}

Le but de ces modules est de faire le calcul de tous les degrés de pertinences des faits de la base concernée. La formule utilisée est expliquée au chapitre 4 (section 4.6: Niveau 1).

II s'agit en fait de quatre modules dont les fonctionnements sont identiques. La seule différence entre ces derniers est la base de faits dans lequel ils interviennent. Chacun de ces quatre modules comprend; en plus des règles pour calculer les différentes normes (ou degrés de pertinence), les deux modules suivants:

- module de recherche du plus grand fait compatible (Compatible),

- module de recherche de faits équivalents (Equivalents). 


\subsection{9: Modules du niveau 2}

Les règles de ces modules gèrent le niveau 2 de gestion. On se trouve à ce niveau lorsque le niveau 1 n'a pas permis de trouver de fait compatible. Par exemple, le système exécute la règle "programme_niveau2" lorsqu'il n'a pas pu définir d'élément de contexte pertinent.

Nous avons, encore une fois, quatre modules identiques (un pour chacune des bases). Chacun de ces derniers comprend les quatre modules suivants:

- module de calcul des normes de compatibilité de niveau 2 (Calcul norme 2),

- module de recherche du fait le plus compatible (Compatible),

- module de recherche des faits équivalents (Equivalents),

- module de vérification concernant le prochain niveau de règles à considérer (Vérif niveau).

Dans un premier temps, cette règle appèle les règles "élargir(programme)" et "élargir(contexte)" qui s'occupent de l'élargissement des intervalles d'acceptabilité des faits. Cette réinitialisation des intervalles de compatibilité permettra de choisir un élément de programme compatible parmi ceux qui ont déjà été choisi à des étapes précédentes. On passe ensuite à la règle "calcul_norme2(programme)" qui fait un nouveau calcul des degrés de pertinence des éléments de programme. Les règles et sous-règles de "calcul_norme2" ne sont pas expliquées ici car ça risquerait d'être long et inutilement technique. Les fonctions utilisées sont toutefois expliquées dans le chapitre 4 où on traite du niveau 2 (section 4.7). On fait ensuite appel aux mêmes règles que pour le niveau 1 afin de terminer la procédure en passant toutefois par la règle "vérif_niveau" (section 7.1.10). 


\subsubsection{0: Module de vérification du niveau suivant}

Ce module réévalue le niveau de règles nécessaire à la poursuite des opérations. Nous avons déjà vu que, après être passé au niveau 2 , il est possible que nous ayons réussi à trouver un fait compatible (chapitre 4). Dans ce cas, le système est renvoyé au niveau 1 pour la poursuite des opérations. Si ce n'est pas le cas, l'impasse est toujours présente; le système a besoin d'une aide extérieure et il passe au niveau 3.

Le principe employé pour cette vérification se base sur les résultats obtenus au niveau 2 par rapport à ceux qui ont été précédemment obtenus au niveau $1 . \mathrm{Si}$ les degrés de pertinence sont les mêmes pour tous les faits de la base, il y a lieu de considérer que l'impasse demeure. Ce module indique alors au système d'aller au niveau 3. Sinon, c'est-à-dire si au moins un fait a obtenu un degré de pertinence différent, on retourne au niveau 1 avec ces nouveaux résultats. Si à ce niveau (niveau 1) les résultats conduisent toujours à l'impasse, alors au prochain passage au niveau 2 il n'y aura plus de différence entre les anciens et nouveaux degrés de pertinence entraînant ainsi le passage au niveau 3 . Sinon, le système continue l'exécution au niveau 1.

\subsubsection{1: Module du niveau 3}

Dans le programme implanté, le passage au niveau 3 ne conduit qu'à la sortie du programme. On aurait pu faire appel à un expert externe (système expert ou un expert humain), ou encore poser plus de questions à l'apprenant afin de vérifier l'état du système, mais ce n'était pas nécessaire pour démontrer la faisabilité du système. 


\subsubsection{2: Module final}

Dans ce module, le système ferme toutes les bases de connaissances et fichiers utilisés de façon à être prêt pour une exécution ultérieure.

\subsubsection{3: Niveau 0}

Au niveau 0 , on fait des changements à l'intérieur de la base de faits dans laquelle on se situe sans faire appel aux autres bases de faits. Ce niveau est de plus celui de l'interaction directe de la base de faits avec l'extérieur. Le module "demander_note" en est un de ce type.

Le module "demander_note" intervient après les choix d'activités d'apprentissages au niveau 1 ou au niveau 2. Dans un premier temps, cette note sert à l'évaluation de l'activité elle-même. En effet, on retourne à la base Activité afin de modifier le degré de pertinence du dernier exercice proposé. Cette note est ensuite utilisée lors des choix subséquents de résultat et programme compatibles.

Un autre module représente une règle de niveau 0 en agissant uniquement sur la base Résultat. II s'agit du module de vérification de la réussite de l'objectif final dont l'unique but est de vérifier si l'usager a réussi la simplification de l'expression booléenne donnée. Si l'usager a réussi, le contrôleur de niveau est mis à 3 afin de provoquer la sortie lors du prochain passage au superviseur. Sinon, la règle "calcul_norme(programme)" échoue, ce qui incite l'exécution de la prochaine règle "calcul_norme(programme)" donc la poursuite normale du processus. 


\subsubsection{4: Niveau (-1)}

En rétroaction (au niveau (-1) de règles), la note obtenue à l'activité permet de corriger le degré de pertinence du contexte considéré. Cette correction peut se faire de plusieurs façons et la façon retenue est la suivante: en partant du principe que si la note est très bonne et que le contexte considéré indique que l'apprenant est rapide, on peut augmenter le degré de pertinence pour cet élément de contexte. $\mathrm{Si}$, par contre, les résultats montrent une très bonne note alors que l'on a affaire (selon le contexte) à un usager lent, on doit diminuer le degré de pertinence pour cet élément de contexte. De plus, on doit diminuer plus (ou augmenter plus) si la note est très faible (ou très forte).

Remarque: Ce niveau (-1) n'est présent qu'après le niveau 0 de la base Activité car c'est seulement dans cette base que le niveau 0 intervient dans notre logiciel. Cependant, il est très raisonnable de penser à cette rétroaction pour toutes les bases. Elle s'effectuerait d'ailleurs exactement de la même manière. On peut donc penser à un système demandant à l'usager quel élément de programme il désire travailler ou encore évaluant à intervalle régulier, par une question ou autre chose, les éléments de contextes considérés.

Nous espérons que ces renseignements sont suffisants pour permettre une compréhension globale du système. Le lecteur obtiendra une meilleure idée des résultats obtenu du logiciel PROLIN en lisant le chapitre qui suit puisqu'il présente les résultats de quelques unes des simulations effectuées à l'aide de ce dernier. Vous trouverez également d'autres résultats de simulations dans la partie $5 \mathrm{du}$ rapport technique et une sortie d'imprimante telle que fourni par PROLIN dans la partie 6 du même rapport. 
CHAPITRE VIII

\section{SIMULATIONS DU PROGRAMME PROLIN}




\section{CHAPITRE VIII}

\section{SIMULATIONS DU PROGRAMME PROLIN}

\section{0: Introduction}

Dans le cadre de cette recherche, plusieurs simulations du programme PROLIN ont été développées. Quinze d'entre elles ont été placées dans des tableau afin d'en permettre une meilleure lisibilité. Dans le présent document, nous présentons quatre (4) de ces résultats de simulations caractéristiques, les autres sommaires sont présentés dans le rapport technique à la partie 5. La partie 6 du même rapport montre une sortie d'imprimante telle qu'obtenue lors de la simulation elle-même. 


\section{1: Sommaire des résultats de 4 simulations de PROLIN}

\section{Cas 1:}

Profil simulé: Etudiant rapide qui obtient des résultats parfaits partout.

Pourcentage visuel: $\quad 0.8$

Pourcentage auditif: $\quad 0.2$

Pourcentage kinesthésique: $\quad 0.0$

Vitesse d'apprentissage: $\quad 0.9$

\begin{tabular}{|c|c|c|c|c|}
\hline & CONTEXTE & ACTIVITE & RESULTAT & PROGRAMME \\
\hline 1 & $\begin{array}{c}\text { 3: L'apprenant est } \\
\text { visuel et rapide }\end{array}$ & $\begin{array}{l}\text { 30: Compléter des } \\
\text { tables de vérité sur les } \\
\text { postulats } \\
\text { note: } 1.0\end{array}$ & $\begin{array}{l}\text { 1: Utilisation des } \\
\text { théorèmes de } \\
\text { l'élément neutre }\end{array}$ & $\begin{array}{l}\text { 2: Introduction à } \\
\text { l'algèbre de Boole }\end{array}$ \\
\hline 2 & $\begin{array}{l}\text { 7: L'apprenant est } \\
\text { kinesthésique et lent }\end{array}$ & $\begin{array}{c}\text { 26: Résoudre } \\
\text { (distributivité du } \\
\text { produit sur la somme } \\
\text { logique) } \\
\text { note: } 1.0\end{array}$ & $\begin{array}{l}\text { 3: Utilisation des } \\
\text { théorèmes de la } \\
\text { distributivité }\end{array}$ & $\begin{array}{l}\text { 13: Théorème de la } \\
\text { distributivité de la } \\
\text { somme sur le produit } \\
\text { logique }\end{array}$ \\
\hline 3 & $\begin{array}{l}\text { 3: L'apprenant est } \\
\text { visuel et rapide }\end{array}$ & $\begin{array}{c}\text { 27: Résoudre } \\
\text { (distributivité de la } \\
\text { somme sur le produit } \\
\text { logique) } \\
\text { note: } 1.0\end{array}$ & $\begin{array}{l}\text { 3: Utilisation des } \\
\text { théorèmes de la } \\
\text { distributivité }\end{array}$ & $\begin{array}{l}\text { 12: Théorème de la } \\
\text { distributivité du produit } \\
\text { sur la somme logique }\end{array}$ \\
\hline 4 & $\begin{array}{l}\text { 3: L'apprenant est } \\
\text { visuel et rapide }\end{array}$ & $\begin{array}{c}\text { 11: Simplifier } \\
\text { (complémentation et } \\
\text { De Morgan) } \\
\text { note: } 1.0\end{array}$ & $\begin{array}{l}\text { 2: Utilisation des } \\
\text { théorèmes de la } \\
\text { complémentation }\end{array}$ & $\begin{array}{l}\text { 14: La première loi de } \\
\text { De Morgan }\end{array}$ \\
\hline 5 & $\begin{array}{l}\text { 6: L'apprenant est } \\
\text { auditif et rapide }\end{array}$ & $\begin{array}{l}\text { 8: Simplifier (élément } \\
\text { neutre et distributivité) } \\
\text { note: } 1.0\end{array}$ & $\begin{array}{l}\text { 5: Simplification } \\
\text { d'expressions } \\
\text { booléennes à } 2 \\
\text { variables }\end{array}$ & $\begin{array}{c}\text { 6: Les théorèmes de } \\
\text { niveau } 2\end{array}$ \\
\hline 6 & $\begin{array}{l}\text { 9: L'apprenant est } \\
\text { kinesthésique et } \\
\text { rapide }\end{array}$ & $\begin{array}{l}\text { 3: Simplifier (élément } \\
\text { neutre, } \\
\text { complémentation et } \\
\text { distributivité) } \\
\text { note: } 1.0\end{array}$ & $\begin{array}{l}\text { 5: Simplification } \\
\text { d'expressions } \\
\text { booléennes à } 2 \\
\text { variables }\end{array}$ & $\begin{array}{l}\text { 1: Simplification de } \\
\text { l'expression } \\
\text { booléenne donnée }\end{array}$ \\
\hline
\end{tabular}




\begin{tabular}{c|c|c|c|c}
\hline 7 & $\begin{array}{c}2: \text { L'apprenant est } \\
\text { visuel et moyen }\end{array}$ & $\begin{array}{c}\text { 1: Simplifier } \\
\text { l'expression donnée } \\
\text { note: } 1.0\end{array}$ & $\begin{array}{c}\text { 8: Utilisation des } \\
\text { postulats }\end{array}$ & \\
\hline
\end{tabular}

Fin de la simulation.

L'apprenant termine sa session par la réussite de l'objectif final car il simplifie correctement l'expression donnée. On remarque que la suite des activités proposées présente une progression régulière dans le niveau de difficulté. PROLIN vérifie d'abord l'acquisition des postulats de base à l'étape 1 en proposant l'activité 30 , puis vérifie la compréhension des opérations et théorèmes en faisant résoudre des expressions booléennes (étapes 2 et 3). Finalement, il propose quelques simplifications demandant l'utilisation de deux théorèmes (étapes 4 et 5), puis de trois théorèmes (étape 6) pour finir avec la simplification de l'expression donnée à l'étape 7. La progression dans le niveau de difficulté des activités présentées est relativement rapide parce que l'étudiant réussit bien en plus d'avoir préalablement été défini rapide. 


\section{Cas 2:}

Profil simulé: Etudiant défini fort mais qui échoue dès le début.

Pourcentage visuel: $\quad 0.8$

Pourcentage auditif: $\quad 0.2$

Pourcentage kinesthésique: $\quad 0.0$

Vitesse d'apprentissage: $\quad 0.8$

\begin{tabular}{c|c|c|c|c} 
& CONTEXTE & ACTIVITE & RESULTAT & PROGRAMME \\
\hline \hline 1 & $\begin{array}{c}\text { 3: L'apprenant est } \\
\text { visuel et rapide }\end{array}$ & $\begin{array}{c}\text { 30: Compléter des } \\
\text { tables de vérité sur les } \\
\text { postulats } \\
\text { note: } 0.0\end{array}$ & $\begin{array}{c}\text { aucun } \\
\text { Niveau 2 }\end{array}$ & \\
\hline 2 & & $\begin{array}{c}1: \text { Simplifier } \\
\text { 'expression donnée } \\
\text { note: } 0.0\end{array}$ & $\begin{array}{c}\text { aucun } \\
\text { Niveau 3 }\end{array}$ & \\
\hline
\end{tabular}

Fin de la simulation.

L'apprenant termine sa session par un fin abrupte des opérations. Le problème ici, c'est que l'étudiant est défini au départ comme étant rapide et fort mais son premier résultat indique exactement le contraire. PROLIN, qui ne trouve pas de résultat compatible avec ce fait passe au niveau 2. On décide ensuite de proposer l'activité finale afin de voir où on en est. Comme la réussite est encore nulle, PROLIN ne trouve toujours pas de résultat compatible. On va donc au niveau 3 d'où la fin des opérations.

On peut conclure ici que l'apprenant a été très mal défini au départ ou encore que les bases de PROLIN devraient être complétée pour couvrir ce cas. 


\section{Cas 3:}

Profil simulé: Etudiant qui débute fort mais dont les résultats diminuent lentement Pourcentage visuel: $\quad 0.8$

Pourcentage auditif: $\quad 0.2$

Pourcentage kinesthésique: $\quad 0.0$

Vitesse d'apprentissage: $\quad 0.8$

\begin{tabular}{|c|c|c|c|c|}
\hline & CONTEXTE & ACTIVITE & RESULTAT & PROGRAMME \\
\hline 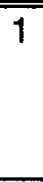 & $\begin{array}{l}\text { 3: L'apprenant est } \\
\text { visuel et rapide }\end{array}$ & $\begin{array}{c}\text { 30: Compléter des } \\
\text { tables de vérité sur les } \\
\text { postulats } \\
\text { note: } 0.9\end{array}$ & $\begin{array}{l}\text { 1: Utilisation des } \\
\text { théorèmes de } \\
\text { l'élément neutre }\end{array}$ & $\begin{array}{l}\text { 2: Introduction à } \\
\text { l'algèbre de Boole }\end{array}$ \\
\hline 2 & $\begin{array}{l}\text { 7: L'apprenant est } \\
\text { kinesthésique et lent }\end{array}$ & $\begin{array}{c}\text { 26: Résoudre } \\
\text { (distributivité du } \\
\text { produit sur la somme } \\
\text { logique) } \\
\text { note: } 0.8\end{array}$ & $\begin{array}{l}\text { 3: Utilisation des } \\
\text { théorèmes de la } \\
\text { distributivité }\end{array}$ & $\begin{array}{l}\text { 14: La première loi de } \\
\text { De Morgan }\end{array}$ \\
\hline 3 & $\begin{array}{l}\text { 3: L'apprenant est } \\
\text { visuel et rapide }\end{array}$ & $\begin{array}{c}\text { 28: Résoudre } \\
\text { (première loi de De } \\
\text { Morgan) } \\
\text { note: } 0.7\end{array}$ & $\begin{array}{c}\text { 4: Utilisation des } \\
\text { théorèmes des lois de } \\
\text { De Morgan }\end{array}$ & $\begin{array}{l}\text { 3: Simplification } \\
\text { d'expressions } \\
\text { booléennes }\end{array}$ \\
\hline 4 & $\begin{array}{l}\text { 6: L'apprenant est } \\
\text { auditif et rapide }\end{array}$ & $\begin{array}{l}\text { 2: Simplifier (ensemble } \\
\text { des théorèmes) } \\
\text { note: } 0.6\end{array}$ & $\begin{array}{l}\text { 2: Utilisation des } \\
\text { théorèmes de la } \\
\text { complémentation }\end{array}$ & $\begin{array}{l}\text { 3: Simplification } \\
\text { d'expressions } \\
\text { booléennes }\end{array}$ \\
\hline 5 & $\begin{array}{c}\text { 9: L'apprenant est } \\
\text { kinesthésique et } \\
\text { rapide }\end{array}$ & $\begin{array}{l}\text { 1: Simplifier } \\
\text { l'expression donnée } \\
\text { note: } 0.5\end{array}$ & $\begin{array}{c}\text { 4: Utilisation des } \\
\text { théorèmes des lois de } \\
\text { De Morgan }\end{array}$ & $\begin{array}{c}\text { 1: Simplification de } \\
\text { l'expression booléenne } \\
\text { donnée }\end{array}$ \\
\hline 6 & $\begin{array}{c}\text { 9: L'apprenant est } \\
\text { kinesthésique et } \\
\text { rapide }\end{array}$ & $\begin{array}{l}\text { 1: Simplifier } \\
\text { l'expression donnée } \\
\text { note: } 0.4\end{array}$ & $\begin{array}{l}\text { 3: Utilisation des } \\
\text { théorèmes de la } \\
\text { distributivité }\end{array}$ & $\begin{array}{c}\text { 1: Simplification de } \\
\text { l'expression booléenne } \\
\text { donnée }\end{array}$ \\
\hline 7 & $\begin{array}{c}\text { 9: L'apprenant est } \\
\text { kinesthésique et } \\
\text { rapide }\end{array}$ & $\begin{array}{l}\text { 1: Simplifier } \\
\text { l'expression donnée } \\
\text { note: } 1.0\end{array}$ & $\begin{array}{l}\text { 8: Utilisation des } \\
\text { postulats }\end{array}$ & \\
\hline
\end{tabular}

Fin de la simulation.

L'apprenant termine cette session par la réussite de l'objectif final même si sa compréhension semble diminuer lentement à chaque pas. PROLIN est 
embarrassé dans cette situation, c'est pourquoi il passe dès l'étape 5 à l'activité finale. Ensuite, ne sachant quoi faire, le système propose encore l'activité finale jusqu'à ce que l'apprenant la réussisse. II est certain que l'apprenant réussira l'activité finale après quelques essais puisque cette dernière comporte cinq choix de réponses (voir le cahier d'activités à l'annexe 6). Le fait de ne disposer que de 33 activités d'apprentissage est sûrement une des causes de cet embarras. 


\section{Cas 4:}

Profil simulé: Etudiant qui présente des difficultés avec la théorie de l'élément neutre.

Pourcentage visuel: $\quad 0.0$

Pourcentage auditif: $\quad 0.8$

Pourcentage kinesthésique: $\quad 0.2$

Vitesse d'apprentissage: $\quad 0.8$

\begin{tabular}{|c|c|c|c|c|}
\hline & CONTEXTE & ACTIVITE & RESULTAT & PROGRAMME \\
\hline 1 & $\begin{array}{l}\text { 6: L'apprenant est } \\
\text { auditif et rapide }\end{array}$ & $\begin{array}{l}\text { 30: Compléter des } \\
\text { tables de vérité sur les } \\
\text { postulats } \\
\text { note: } 1.0\end{array}$ & $\begin{array}{l}\text { 1: Utilisation des } \\
\text { théorèmes de } \\
\text { l'élément neutre }\end{array}$ & $\begin{array}{l}\text { 2: Introduction à } \\
\text { l'algèbre de Boole }\end{array}$ \\
\hline 2 & $\begin{array}{c}\text { 7: L'apprenant est } \\
\text { kinesthésique et lent }\end{array}$ & $\begin{array}{c}\text { 26: Résoudre } \\
\text { (distributivité du } \\
\text { produit sur la somme } \\
\text { logique) } \\
\text { note: } 1.0\end{array}$ & $\begin{array}{l}\text { 3: Utilisation des } \\
\text { théorèmes de la } \\
\text { distributivité }\end{array}$ & $\begin{array}{l}\text { 13: Théorème de la } \\
\text { distributivité de la } \\
\text { somme sur le produit } \\
\text { logique }\end{array}$ \\
\hline 3 & $\begin{array}{l}\text { 6: L'apprenant est } \\
\text { auditif et rapide }\end{array}$ & $\begin{array}{c}\text { 27: Résoudre } \\
\text { (distributivité de la } \\
\text { somme sur le produit } \\
\text { logique) } \\
\text { note: } 1.0\end{array}$ & $\begin{array}{l}\text { 3: Utilisation des } \\
\text { théorèmes de la } \\
\text { distributivité }\end{array}$ & $\begin{array}{l}\text { 12: Théorème de la } \\
\text { distributivité du produit } \\
\text { sur la somme logique }\end{array}$ \\
\hline 4 & $\begin{array}{l}\text { 6: L'apprenant est } \\
\text { auditif et rapide }\end{array}$ & $\begin{array}{c}\text { 11: Simplifier } \\
\text { (complémentation et } \\
\text { De Morgan) } \\
\text { note: } 0.0\end{array}$ & $\begin{array}{l}\text { 1: Utilisation des } \\
\text { théorèmes de } \\
\text { l'élément neutre }\end{array}$ & $\begin{array}{l}\text { 12: Théorème de la } \\
\text { distributivité du produit } \\
\text { sur la somme logique }\end{array}$ \\
\hline 5 & $\begin{array}{l}\text { 9: L'apprenant est } \\
\text { kinesthésique et } \\
\text { rapide }\end{array}$ & $\begin{array}{c}\text { 4: Simplifier (élément } \\
\text { neutre, } \\
\text { complémentation et } \\
\text { De Morgan) } \\
\text { note: } 1.0\end{array}$ & $\begin{array}{l}\text { 2: Utilisation des } \\
\text { théorèmes de la } \\
\text { complémentation }\end{array}$ & $\begin{array}{l}\text { 3: Simplification } \\
\text { d'expressions } \\
\text { booléennes }\end{array}$ \\
\hline 6 & $\begin{array}{l}\text { 3: L'apprenant est } \\
\text { visuel et rapide }\end{array}$ & $\begin{array}{l}\text { 2: Simplifier (ensemble } \\
\text { des théorèmes) } \\
\text { note: } 0.0\end{array}$ & $\begin{array}{c}\text { 4: Utilisation des } \\
\text { théorèmes des lois de } \\
\text { De Morgan }\end{array}$ & $\begin{array}{l}\text { 10: Théorème de la } \\
\text { complémentation du } \\
\text { produit logique }\end{array}$ \\
\hline 7 & $\begin{array}{l}\text { 9: L'apprenant est } \\
\text { kinesthésique et } \\
\text { rapide }\end{array}$ & $\begin{array}{l}\text { 20: Tables de vérité } \\
\text { (distributivité) } \\
\text { note: } 1.0\end{array}$ & $\begin{array}{l}\text { 1: Utilisation des } \\
\text { théorèmes de } \\
\text { l'élément neutre }\end{array}$ & $\begin{array}{l}\text { 10: Théorème de la } \\
\text { complémentation du } \\
\text { produit logique }\end{array}$ \\
\hline 8 & $\begin{array}{c}\text { 8: L'apprenant est } \\
\text { kinesthésique et } \\
\text { moyen }\end{array}$ & $\begin{array}{l}17: \text { Compléter des } \\
\text { énoncés de théorèmes } \\
\text { note: } 0.7\end{array}$ & $\begin{array}{l}\text { 7: Simplification de } \\
\text { l'expression booléenne } \\
\text { prédéfinie }\end{array}$ & $\begin{array}{l}\text { 3: Simplification } \\
\text { d'expressions } \\
\text { booléennes }\end{array}$ \\
\hline
\end{tabular}




\begin{tabular}{|c|c|c|c|c|}
\hline 9 & $\begin{array}{l}\text { 6: L'apprenant est } \\
\text { auditif et rapide }\end{array}$ & $\begin{array}{l}\text { 16: Simplifier (lois e De } \\
\text { Morgan) } \\
\text { note: } 0.0\end{array}$ & $\begin{array}{l}\text { 5: Simplification } \\
\text { d'expressions } \\
\text { booléennes à } 2 \\
\text { variables }\end{array}$ & $\begin{array}{l}\text { 13: Théorème de la } \\
\text { distributivité de la } \\
\text { somme sur le produit } \\
\text { logique }\end{array}$ \\
\hline 10 & $\begin{array}{l}\text { 9: L'apprenant est } \\
\text { kinesthésique et } \\
\text { rapide }\end{array}$ & $\begin{array}{l}\text { 1: Simplifier } \\
\text { l'expression donnée } \\
\text { note: } 0.0\end{array}$ & $\begin{array}{l}\text { 5: Simplification } \\
\text { d'expressions } \\
\text { booléennes à } 2 \\
\text { variables }\end{array}$ & $\begin{array}{l}\text { 1: Simplification de } \\
\text { l'expression booléenne } \\
\text { donnée }\end{array}$ \\
\hline 11 & $\begin{array}{l}\text { 3: L'apprenant est } \\
\text { visuel et rapide }\end{array}$ & $\begin{array}{c}\text { 1: Simplifier } \\
\text { l'expression donnée } \\
\text { note: } 0.7\end{array}$ & $\begin{array}{l}\text { 5: Simplification } \\
\text { d'expressions } \\
\text { booléennes à } 2 \\
\text { variables }\end{array}$ & $\begin{array}{c}\text { 1: Simplification de } \\
\text { l'expression booléenne } \\
\text { donnée }\end{array}$ \\
\hline 12 & $\begin{array}{l}\text { 6: L'apprenant est } \\
\text { auditif et rapide }\end{array}$ & $\begin{array}{c}\text { 30: Compléter des } \\
\text { tables de vérité sur les } \\
\text { postulats } \\
\text { note: } 1.0\end{array}$ & $\begin{array}{l}\text { 1: Utilisation des } \\
\text { théorèmes de } \\
\text { l'élément neutre }\end{array}$ & $\begin{array}{l}\text { 2: Introduction à } \\
\text { l'algèbre de Boole }\end{array}$ \\
\hline 13 & $\begin{array}{l}\text { 7: L'apprenant est } \\
\text { kinesthésique et lent }\end{array}$ & $\begin{array}{c}\text { 26: Résoudre } \\
\text { (distributivité du } \\
\text { produit sur la somme } \\
\text { logique) } \\
\text { note: } 1.0\end{array}$ & $\begin{array}{l}\text { 3: Utilisation des } \\
\text { théorèmes de la } \\
\text { distributivité }\end{array}$ & $\begin{array}{l}\text { 13: Théorème de la } \\
\text { distributivité de la } \\
\text { somme sur le produit } \\
\text { logique }\end{array}$ \\
\hline 14 & $\begin{array}{l}\text { 6: L'apprenant est } \\
\text { auditif et rapide }\end{array}$ & $\begin{array}{c}\text { 27: Résoudre } \\
\text { (distributivité de la } \\
\text { somme sur le produit } \\
\text { logique) } \\
\text { note: } 1.0\end{array}$ & $\begin{array}{l}\text { 3: Utilisation des } \\
\text { théorèmes de la } \\
\text { distributivité }\end{array}$ & $\begin{array}{l}\text { 12: Théorème de la } \\
\text { distributivité du produit } \\
\text { sur la somme logique }\end{array}$ \\
\hline 15 & $\begin{array}{l}\text { 6: L'apprenant est } \\
\text { auditif et rapide }\end{array}$ & $\begin{array}{c}\text { 11: Simplifier } \\
\text { (complémentation et } \\
\text { De Morgan) } \\
\text { note: } 1.0\end{array}$ & $\begin{array}{l}\text { 2: Utilisation des } \\
\text { théorèmes de la } \\
\text { complémentation }\end{array}$ & $\begin{array}{c}\text { 14: La première loi de } \\
\text { De Morgan }\end{array}$ \\
\hline 16 & $\begin{array}{l}\text { 9: L'apprenant est } \\
\text { kinesthésique et } \\
\text { rapide }\end{array}$ & $\begin{array}{l}\text { 8: Simplifier (élément } \\
\text { neutre et distributivité) } \\
\text { note: } 0.3\end{array}$ & $\begin{array}{l}\text { 4: Utilisation des } \\
\text { théorèmes des lois de } \\
\text { De Morgan }\end{array}$ & $\begin{array}{l}\text { 11: Théorème de la } \\
\text { complémentation de la } \\
\text { somme logique }\end{array}$ \\
\hline 17 & $\begin{array}{l}\text { 6: L'apprenant est } \\
\text { auditif et rapide }\end{array}$ & $\begin{array}{l}\text { 20: Tables de vérité } \\
\text { (distributivité) } \\
\text { note: } 1.0\end{array}$ & $\begin{array}{l}\text { 2: Utilisation des } \\
\text { théorèmes de la } \\
\text { complémentation }\end{array}$ & $\begin{array}{l}\text { 10: Théorème de la } \\
\text { complémentation du } \\
\text { produit logique }\end{array}$ \\
\hline 18 & $\begin{array}{l}\text { 3: L'apprenant est } \\
\text { visuel et rapide }\end{array}$ & $\begin{array}{c}\text { 12: Simplifier } \\
\text { (distributivité et De } \\
\text { Morgan) } \\
\text { note: } 0.6\end{array}$ & $\begin{array}{l}\text { 5: Simplification } \\
\text { d'expressions } \\
\text { booléennes à } 2 \\
\text { variables } \\
\end{array}$ & $\begin{array}{l}\text { 13: Théorème de la } \\
\text { distributivité de la } \\
\text { somme sur le produit } \\
\text { logique }\end{array}$ \\
\hline 19 & $\begin{array}{l}\text { 6: L'apprenant est } \\
\text { auditif et rapide }\end{array}$ & $\begin{array}{l}\text { 19: Tables de vérité } \\
\text { (complémentation) } \\
\text { note: } 1.0\end{array}$ & $\begin{array}{l}\text { 3: Utilisation des } \\
\text { théorèmes de la } \\
\text { distributivité }\end{array}$ & $\begin{array}{c}\text { 14: La première loi de } \\
\text { De Morgan }\end{array}$ \\
\hline 20 & $\begin{array}{l}\text { 3: L'apprenant est } \\
\text { visuel et rapide }\end{array}$ & $\begin{array}{c}\text { 6: Simplifier } \\
\text { (complémentation, } \\
\text { distributivité et De } \\
\text { Morgan) } \\
\text { note: } 1.0\end{array}$ & $\begin{array}{l}\text { 5: Simplification } \\
\text { d'expressions } \\
\text { booléennes à } 2 \\
\text { variables }\end{array}$ & $\begin{array}{c}\text { 1: Simplification de } \\
\text { l'expression booléenne } \\
\text { donnée }\end{array}$ \\
\hline
\end{tabular}




\begin{tabular}{|c|c|c|c|c|}
\hline 21 & $\begin{array}{c}\text { 9: L'apprenant est } \\
\text { kinesthésique et } \\
\text { rapide }\end{array}$ & $\begin{array}{l}\text { 1: Simplifier } \\
\text { l'expression donnée } \\
\text { note: } 0.0\end{array}$ & $\begin{array}{l}\text { 5: Simplification } \\
\text { d'expressions } \\
\text { booléennes à } 2 \\
\text { variables } \\
\end{array}$ & $\begin{array}{c}\text { 1: Simplification de } \\
\text { l'expression booléenne } \\
\text { donnée }\end{array}$ \\
\hline 22 & $\begin{array}{c}\text { 9: L'apprenant est } \\
\text { kinesthésique et } \\
\text { rapide }\end{array}$ & $\begin{array}{l}\text { 1: Simplifier } \\
\text { l'expression donnée } \\
\text { note: } 0.7\end{array}$ & $\begin{array}{l}\text { 8: Utilisation des } \\
\text { postulats }\end{array}$ & $\begin{array}{l}\text { 2: Introduction à } \\
\text { l'algèbre de Boole }\end{array}$ \\
\hline 23 & $\begin{array}{c}\text { 8: L'apprenant est } \\
\text { kinesthésique et } \\
\text { moyen }\end{array}$ & $\begin{array}{l}\text { 17: Compléter des } \\
\text { énoncés de théorèmes } \\
\text { note: } 0.8\end{array}$ & $\begin{array}{l}\text { 5: Simplification } \\
\text { d'expressions } \\
\text { booléennes à } 2 \\
\text { variables }\end{array}$ & $\begin{array}{c}\text { 15: La deuxième loi de } \\
\text { De Morgan }\end{array}$ \\
\hline 24 & $\begin{array}{c}\text { 9: L'apprenant est } \\
\text { kinesthésique et } \\
\text { rapide }\end{array}$ & $\begin{array}{l}\text { 9: Simplifier (élément } \\
\text { neutre et De Morgan) } \\
\text { note: } 0.6\end{array}$ & $\begin{array}{l}\text { 2: Utilisation des } \\
\text { théorèmes de la } \\
\text { complémentation }\end{array}$ & $\begin{array}{l}\text { 14: La première loi de } \\
\text { De Morgan }\end{array}$ \\
\hline 25 & $\begin{array}{c}\text { 9: L'apprenant est } \\
\text { kinesthésique et } \\
\text { rapide }\end{array}$ & $\begin{array}{l}\text { 16: Simplifier (lois de } \\
\text { De Morgan) } \\
\text { note: } 1.0\end{array}$ & $\begin{array}{l}\text { 2: Utilisation des } \\
\text { théorèmes de la } \\
\text { complémentation }\end{array}$ & $\begin{array}{l}\text { 12: Théorème de la } \\
\text { distributivité du produit } \\
\text { sur la somme logique }\end{array}$ \\
\hline 26 & $\begin{array}{l}\text { 3: L'apprenant est } \\
\text { visuel et rapide }\end{array}$ & $\begin{array}{l}\text { 3: Simplifier (élément } \\
\text { neutre, } \\
\text { complémentation et } \\
\text { distributivité) } \\
\text { note: } 1.0\end{array}$ & $\begin{array}{l}\text { 5: Simplification } \\
\text { d'expressions } \\
\text { booléennes à } 2 \\
\text { variables }\end{array}$ & $\begin{array}{l}\text { 13: Théorème de la } \\
\text { distributivité de la } \\
\text { somme sur le produit } \\
\text { logique }\end{array}$ \\
\hline 27 & $\begin{array}{l}\text { 6: L'apprenant est } \\
\text { auditif et rapide }\end{array}$ & $\begin{array}{l}\text { 2: Simplifier (ensemble } \\
\text { des théorèmes) } \\
\text { note: } 1.0\end{array}$ & $\begin{array}{c}\text { 5: Simplification } \\
\text { d'expressions } \\
\text { booléennes à } 2 \\
\text { variables } \\
\end{array}$ & $\begin{array}{l}\text { 9: Théorème de } \\
\text { l'élément neutre de la } \\
\text { somme logique }\end{array}$ \\
\hline 28 & $\begin{array}{c}\text { 9: L'apprenant est } \\
\text { kinesthésique et } \\
\text { rapide }\end{array}$ & $\begin{array}{c}\text { 1: Simplifier } \\
\text { l'expression donnée } \\
\text { note: } 1.0\end{array}$ & $\begin{array}{l}\text { 8: Utilisation des } \\
\text { postulats }\end{array}$ & \\
\hline
\end{tabular}

Fin de la simulation.

L'apprenant termine sa session par la réussite de l'objectif final mais le problème ici, c'est que l'étudiant a des difficultés avec un théorème de niveau 1 , donc un des plus simples et pas du tout avec les autres. PROLIN présente donc une difficulté au départ à définir le problème (comme un humain). L'étudiant commence à comprendre le théorème de l'élément neutre lorsqu'il fait l'activité 17 où on le lui explique. Après cela, ça va de mieux en mieux et il termine sa leçon cinq étapes plus tard à l'étape 28. 
On remarque ici le comportement "intelligent" de PROLIN qui cherche d'abord à évaluer le problème puis apporte le correctif nécessaire.

\section{2: Conclusion}

Beaucoup plus de simulations ont été faites avec PROLIN et tous les résultats allaient dans le même sens que les 15 cas présentés ici. lls démontrent la pertinence des niveaux de règles tels qu'implantés puisqu'il arrive dans environ $75 \%$ des cas que le passage au niveau 2 de règles résolve un problème rencontré au niveau 1 de règles. PROLIN n'est pas parfait, ce n'est qu'un début, ces simulations nous ont toutefois permis de constater des faits importants concernant PROLIN et le modèle préconisé.

D'abord, on se rend immédiatement compte que l'entrée des paramètres psychologiques de l'étudiant au début est primordiale. Si cette dernière est incorrecte, c'est toute la suite des décisions qui se trouve perturbée. Ainsi, le cas 1 étant bien définis au départ, on trouve une progression efficace dans la difficulté des activités proposées. C'est une progression qui respecte le rythme de l'apprenant tout en conduisant bien à l'objectif final. Pour le cas 2, ce n'est pas le cas; l'étudiant est mal défini au départ et PROLIN présente des difficultés. 
Une autre faiblesse de PROLIN est causée par le manque d'activités d'apprentissage. On pourrait trouver un net avantage à disposer de plusieurs activités d'un même type puisque PROLIN ne reprendra que très rarement une activité déjà proposée. Ce fait aurait probablement tiré PROLIN d'embarras dans le cas 3.

Le cas 4 retenu est caractérisé par l'instabilité de l'apprenant. Ce dernier répond tantôt bien, tantôt mal à des activités de même niveau, ou encore il répond bien à une activité de niveau supérieur et mal à une activité de niveau inférieur (donc plus facile). Dans ce cas PROLIN présente un comportement simulant celui d'un humain, donc un peu comme un professeur.

L'objectif d'obtenir un système informatique de gestion général de ressources (humaines) simulant un comportement intelligent semble bien être atteint à la lumière des résultats que nous avons obtenus. II reste toutefois, bien du travail à faire pour arriver à un système complet et autonome. Nous en sommes conscient, c'est pourquoi nous pouvons affirmer que ce n'est qu'un début. 
CONCLUSION ET RECOMMANDATIONS 


\section{CONCLUSION ET RECOMMANDATIONS}

Le présent projet se proposait de modéliser un système informatique de gestion générale de ressources (humaines ou non) simulant un comportement humain. Pour cela, nous avons d'abord cherché les qualités nécessaires à tout modèle de gestion de situations d'apprentissage. Ces dernières ont été déduites de modèles existants dans la littérature pédagogique (chapitre 1).

Par la suite, l'analyse des systèmes tutoriels existants nous a permis de connaître ce qui se fait déjà en informatique dans les milieux de l'enseignement. De là, nous avons dégagé un modèle conceptuel minimal et général. Pour aider le système dans ses prises de décisions, cette structure minimale requiert un programme contenant les objectifs pédagogiques à atteindre et un modèle étudiant contenant les acquis de l'apprenant.

Après une étude de différents modèles étudiants, nous avons opté pour un modèle qui considère la connaissance de l'apprenant comme un sous-ensemble de celle de l'expert. Nous avons décidé d'utiliser le modèle d'André Ouellet, comme modèle de base. L'utilisation de ce dernier s'est avéré un bon choix mais difficilement programmable sur un micro-ordinateur conventionnel; il a donc fallu apporter de légères modifications. C'est ainsi que les quatres ensembles proposés par Ouellet (Intervention pédagogique, Contexte, Processus d'apprentissage et Résultats) sont devenus les bases de connaissances Programme, Contexte, Activité et Résultat dont chacune peut elle-même constituer un système expert. De même, le dialogue suggéré par Ouellet (chapitre 3) ne pouvant s'appliquer tel quel, 
nous avons établi une nouvelle structure permettant une gestion efficace du système en utilisant les bases prises deux à deux. Puis, pour éliminer toute possibilité d'impasse, nous avons implanté des niveaux supérieurs de règles.

Chacune des bases peut avoir des contenus et des structures différentes des autres, ce qui permet plus de latitude au système. Par exemple:

- dans une situation d'enseignement, il suffirait de modifier une ou deux de ces bases pour enseigner un autre sujet;

- dans une situation de gestion de projet, on pourrait utiliser des bases d'importances inégales.

Pour confirmer ces affirmations, nous avons développé deux exemples. Le premier concerne l'apprentissage de l'algèbre élémentaire de Boole et a donné naissance au logiciel PROLIN. Le second traite de la gestion d'un bras manipulateur (robotique) et démontre les possibilités de généralisation du système.

En plus de montrer l'efficacité du modèle, les expérimentations de PROLIN ont permis de découvrir un comportement qui ressemble beaucoup à celui d'un humain (chapitre 8). Les résultats des simulations montrent que l'utilisation des niveaux de règles tels que définis est efficace. En effet, dans les situations d'impasse au niveau 1 , il arrive environ trois fois sur quatre qu'on réussisse à poursuivre les opérations après le passage au niveau 2. Les résultats obtenus montrent cependant certains problèmes qui ne sont toutefois pas liés à la structure même du modèle mais plutôt à ce qui reste à faire.

On pourrait donc compléter le logiciel PROLIN en travaillant au niveau des points faibles du système actuel et faire les recommandations suivantes:

- Ajouter de véritables systèmes experts associés à chacune des bases de faits. Le fait d'avoir imposé une structure fixe pour chacune des bases a pour effet de diminuer l'adaptabilité du système. L'ajout de systèmes experts à ces niveaux permettrait au système de prendre des décisions plus éclairées concernant le choix de faits dans chacune des bases entraînant une gestion plus efficace des contre- 
performances de l'apprenant tout en évitant probablement de proposer deux fois de suite la même activité à l'apprenant.

- Consulter des experts dans chacun des domaines concernés pour fixer les limites inférieures et supérieures d'acceptations des faits compatibles à l'intérieur de chacune des bases. Ceci permettrait une meilleure prise de décision par le système. De même, les rétrécissements et élargissements des intervalles de compatibilité en cours de processus gagneraient à être gérés de façon plus stricte, c'est-à-dire dépendant de chacun des faits et des évaluations qui s'y rapportent.

- Consulter des experts en interaction des bases pour fixer les différentes normes de compatibilité. Cette consultation améliorenait probablement les prises de décisions du logiciel; les normes présentes dans PROLIN ont été déterminées au départ selon des critères faisant appel seulement au bon sens de l'auteure.

- Ajouter un grand nombre d'activités d'apprentissage. Ceci permettrait une plus grande variété d'action et offrirait au système l'opportunité de poser plus de questions sur un même sujet. Mieux encore, l'ajout d'un générateur d'activités qui en forgerait sur mesure selon la demande de PROLIN procurerait de très larges possibilités et beaucoup de souplesse.

- Ajouter un analyseur automatique des réponses de l'apprenant. Cela permettrait au système de faire des évaluations plus sophistiquées des apprentissages réels de l'usager.

- Ajouter des règles formant un système expert au niveau du superviseur. Ce dernier pourrait ainsi prendre des décisions plus importantes. Le superviseur présent dans le logiciel PROLIN gère actuellement le système de façon minimale: il ne peut prendre que des décisions très superficielles. L'ajout de règles à ce niveau permettrait, entre autre, de ne pas toujours commander la fin des opérations lors de la rencontre de problèmes au niveau 2 comme c'est le cas présentement, mais de permettre à un expert (résident ou externe) de résoudre le problème. 
- Implanter le niveau (-1) de règles pour chacune des quatre bases et doubler cette implantation d'une évaluation externe de niveau 0 . Le système pourrait ainsi s'autocorriger continuellement. Actuellement le niveau (-1) de règles n'est implanté qu'à la base Activité afin de valider et reconsidérer les normes de la base Contexte mais nous savons qu'il serait tout aussi efficace et utile pour les autres bases.

- Implanter le niveau (-2) de règles serait également intéressant. On pourrait même penser à un niveau (-2) plus sophistiqué utilisant à la fois les résultats de deux bases comme au niveau 1 mais en rétroaction pour corriger des degrés de pertinence déjà calculés.

Comme nous venons de le constater, nous avons obtenu un modèle très riche et applicable dans maintes situations mais il reste encore beaucoup de chemin à parcourir avant de finaliser un tel système. La majeure partie des améliorations demandant les connaissances de nombreux experts, beaucoup de collaboration et travaux d'équipes seront nécessaires à la poursuite de ce projet. Nous pensons toutefois qu'il s'agit d'un pas dans la bonne direction et qu'il vaudrait la peine de continuer à travailler dans ce sens. 


\section{CONTENU DU RAPPORT TECHNIQUE}

Partie 1: Listage du programme PROLIN

Partie 2: Les fichiers de PROLIN

2.1: Le fichier "PCAR.ARI"

2.2: Le fichier "NORMES.ARI"

Partie 3: Les contenus des bases de connaissances

3.1: La base Programme

3.2: La base Contexte

3.3: La base Activité

3.4: La base Résultat

Partie 4: Le cahier d'activités de PROLIN

Partie 5: Simulations de PROLIN

Partie 6: Sortie d'imprimante

Remarque: Le rapport technique dont il est question ici est disponible à la bibliothèque de l'Université du Québec à Chicoutimi, à la bibliothèque centrale de L'Université du Québec et auprès des directeurs de recherche: Jean-Joël Vonarburg (département des sciences appliquées) et Richard Tremblay (département d'informatique et mathématique), tous deux à l'Université du Québec à Chicoutimi. 


\section{BIBLIOGRAPHIE}

[01] ANDERSON J.R., Human Associative Memory, Wiley, New-York, 1974.

[02] ANDERSON J.R., Cognitive Psychology and its Implications, Freeman, San Francisco, 1980.

[03] ATTNEAVE F., "Transfer of Experience with Class Schemata", 1957, Journal of Experimental Psychology, Number 54, pp.81-88.

[04] BLOOM Benjamin S., Caractéristiques individuelles et apprentissage scolaire, Editions LABOR, Bruxelles, 1979.

[05] BRIEN Robert, Design pédagogique, introduction à l'approche de Gané et Briggs, Les éditions Saint-Yves Inc., Ottawa, Canada, 1981.

[06] BURTON, BROWN, "An Investigation of Computer Coaching for Informal Learning Activities", International Journal of Man Machine Studies, 1979 , volume 11, pp.5-24.

[07] CAMPBELL Don G., Introduction to the Musical Brain, Magnamusic-Baton, StLouis, 1983.

[08] CHAPOUTIER Georges, Introduction au fonctionnement du système nerveux: codage et traitement de l'information, Medsi, Paris, 1982.

[09] CLANCEY William J., "GUIDON", Journal of Computer-Based Istruction, Summer 1983, volume 10, number 1 and 2, pp.8-15. 
[10] CLANCEY W.J., Knowledge-Based Tutoring: The Guidon Program, MIT Press, Cambridge, 1987.

[11] DEPOVER Christian, L'ordinateur média d'enseignement: Un cadre conceptuel, De Boeck-Wesmael, 1987.

[12] DESPINS Jean-Paul, "Connaître les styles d'apprentissage pour mieux respecter les façons d'apprendre des enfants", Vie Pédagogique, Novembre 1985, Volume 39, pp.10-15.

[13] DOWNS R.M., Image and Environment: Cognitive and Spatial Behavior, Aldine Publisher, Chicago, 1973.

[14] FAKLMAN Scott E., NETL: a System for Representing and Using Real World Knowledge, MIT Press, Cambridge, 1972.

[15] FINDLER Nicholas V., Associative Networks: Representation and Use Knowledge by Computers, Academic Press, New-York, 1979.

[16] FUGERE B.J., TREMBLAY R., GELEYN L., "A Model for Developing a Tutoring System in Music", Proceeding of AAAl-88. First Workshop on Al and Music, St-Paul, Minnessota, USA, August 1988.

[17] GAGNE Robert M., Les principes fondamentaux de l'apprentissage, Traduit par Robert Brien et Raymond Paquin, Les Editions HRW Ltée, 1976.

[18] GAGNE R.M., Conditions of Learning, Holt Rinehart, New-York, 1971.

[19] GELEYN J.E. Daniel, Solid Modeling Application for Robotic Assembly Simulation, Thesis submitted in partial fulfillment for the 
degree of master of ingineering, Royal Military College of Canada, Kingston, Ontario, 1988.

[20] GIBSON James J., The Senses considered as Perceptual Systems, Houghton Miffin Company, Boston, 1966.

[21] GLASER Robert, Education and Thinking. The Role of Knowledge, American Psychologist, volume 39, number 2, pp.93-104.

[22] HEINES J., "Basic Concepts in Knowledge Based Systems", Machine Mediated Learning, 1983, volume 1 number 1, pp.65-95.

[23] JUDD W.A., GLASER R., "Response Latency as a Function of Training Method, Information Level, Acquisition and Overlearning", Journal of Educational Psychology Monographs, 1969, volume 60 , number 4 , part 2 .

[24] KAPLAN S., Cognition and Environment: Functioning an Uncertain World, Praeger, New-York, 1982.

[26] KASS Robert, "Student Modeling in Intelligent Tutoring Systems - Implications for User Modeling", User Models in Dialog Systems, Alfred Kobsa, Wolfgang Wahlster editors, 1986, pp.386-410.

[27] LAMONTAGNE Claude, Vers une pratique du profil d'apprentissage, Institut de recherche sur le profil d'apprentissage.

[28] LE MOIGNE Jean-Louis, La théorie du système général (théorie de la modélisaton), Presses universitaires de France, 1977.

[29] LESGOLG Alan, "Toward a Theory of Curriculum for Use in Designing Intelligent Instructional Systems", Learning Research and Development Center, University of Pittsburg. 
[30] LEWIS R., "Pedagogical Issues in Designing Programs", Mocrocomputers in Secondary Education, Issues and Technics, Edited by J.A.M. Howe and P.M. Ross, Kogan Page, London, 1981, pp.42-51.

[31] LINDSAY, NORMAN, Traitement de l'information et comportement humain, Ed. Etudes Vivantes, 1980.

[32] LOGAN Robert S., "A State-of-Art Assessment of Instructional Systems Development", Issues in Instructional Systems Development, Edited by Harold F. O'Neil Jr., Academic Press, 1979, pp.120.

[33] MICHAELS C.F., Direct Perception, Prentice-Hall, Englewood Cliffs, 1981.

[34] MURRENS Marc W.F., "La gestion du modèle de l'apprenant dans le système de E.I.A.O. "G"', ICO, Intelligence Artificielle et Sciences Cognitives au Québec, Février 1989, volume 1, numéro 2.

[35] OLSON D.R., Spatial Cognition: the Structure and Development of Mental Representation of Spatials Relations, Erlbaum, Hillsdale, 1983.

[36] O'SHEA Tim, Learning and Teaching with Computers: Artificial Intelligence in Education, Harvester Press, Brighton, 1983.

[37] OUELLET André, L'évaluation créative: une approche systémique des valeurs, Presses de l'Université du Québec, Québec, Canada, 1983.

[38] PAPERT Seymour, Jaillissement de l'esprit, Ordinateur et apprentissage, Flammarion, 1981. 
[39] PIAGET Jean, Les mécanismes perceptifs, Presses universitaires de France, 1961.

[40] PIAGET Jean, Où va l'éducation?, Denoel/Gonthier, 1972.

[41] PROULX Jean, Aide à l'apprentissage: un modèle systémique d'intervention (Tome 1), Collège de Trois-Rivières, 1989.

[42] PROVOST Guy, "Inventaire des qualités d'un système d'enseignement", Les systèmes d'enseignement et apprentissage. Conseil interuniversitaire des professeurs en technologie éducative, Les publications audiovisuelles, 1981.

[43] RICH Elaine, Intelligence artificielle, Masson, Paris, 1983.

[44] RUMELHART D.E., Mc CLELAND J.L., "An Interactive Activation Model of Context Effect in Letter Perception", Psychological Review, 1982, volume 89 , number 1, pp.60-94.

[45] RUSHBY Nick, "A Knowledge-engineering Approach to Instructional Design", The Computer Journal, 1986, volume 19, number 5, pp.385388.

[46] SHIRAI Y., Intelligence artificielle: concepts, techniques et applications, Eyrolles, Paris, 1987.

[47] SKINNER B.F., L'analyse expérimentale du comportement. Un essai théorique, Charles Dessart éditeur, Bruxelles, 1971.

[48] SLEEMAN D., Intelligent Tutoring Systems, Academic Press, Toronto, 1982.

[49] SOLOWAY, "Meno II: An Al Based Programming Tutor", Journal of Computerbased Instruction, 1983, volume 10, number 1, pp.20-34. 
[50] SMITH Frank, La compréhension et l'apprentissage. Un cadre de référence pour l'enseignement, Traduit et adapté par Alain Vézina, Les éditions HRW Ltée, 1979.

[51] SMITH P.R., BLANDFORD C., "Graphical design of teaching simulations", Microcomputers in Secondary Education. Issues and Techniques, Edited by J.A.M. Howe and P.M. Ross, Kogan Page, London, 1981.

[52] SUTTON R.S., BARTO A.G., "Toward a Modern Theory of Adaptive Networks: Expectation and Prediction", Psychological Review, 1981, volume 88 , number 2 , pp.135-170.

[53] THERIAULT Jacqueline, "La pensée d'Asahel Woodruff sur l'apprentissage scolaire", Recueil de textes: Psychologie de l'apprentissage (approche cognitive),

[54] THERIAULT Jacqueline, "L'apprentissage selon Ausubel", Notes complémentaires: Psychologie de l'apprentissage (approche cognitive).

[55] ZEILER Bernard P., Theory of Modelling and Simulation, Wiley, New-York, 1976. 


\section{ANNEXE 1}

INTEGRATION DE DEUX ROLES POSSIBLES DE L'ORDINATEUR DANS UNE SITUATION D'ENSEIGNEMENT 


\section{ANNEXE 1 \\ INTEGRATION DE DEUX ROLES POSSIBLES DE L'ORDINATEUR DANS UNE SITUATION D'ENSEIGNEMENT}

Le but de cette annexe est de montrer l'avantage de l'unification de deux rôles de l'ordinateur dans un contexte d'enseignement. Cette unification permettra de déduire un nouveau système tuteur à deux niveaux qui offrira la possibilité d'une gestion plus complète de situations d'apprentissage.

Lewis [30] distingue deux rôles possibles de l'ordinateur dans l'enseignement. L'ordinateur peut ainsi agir comme remplaçant de l'enseignant auprès de l'apprenant. Son rôle prédominant devient alors celui du didacticien. On peut le résumer par la figure qui suit. 


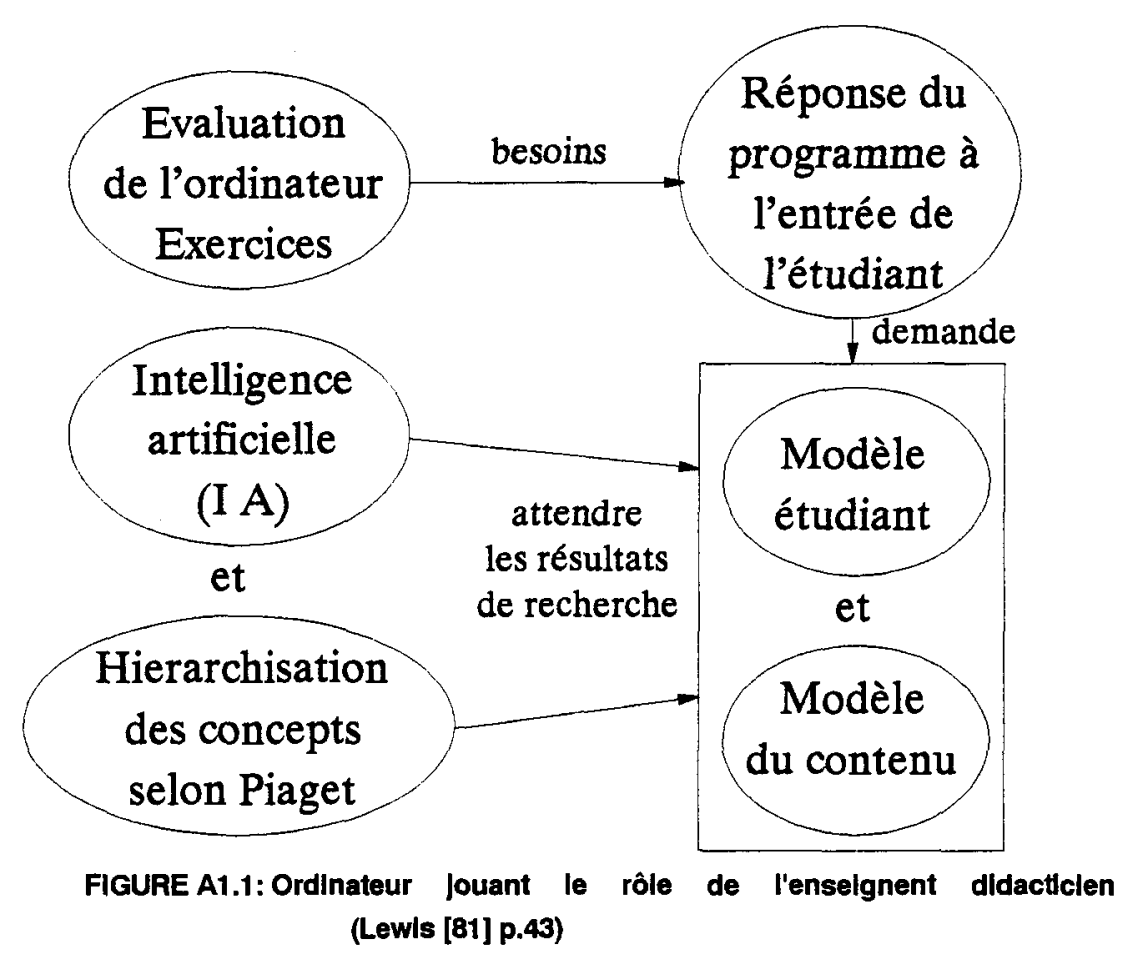

Ce rôle du tuteur est particulièrement intéressant lorsqu'on se trouve en situation de manque de personnel enseignant. C'est un système qui se veut complet en soit et qui pourrait, dans une certaine mesure, combler le manque de professeur. Toutefois, un tel système, aussi complet soit-il, ne saura sûrement pas répondre à tous les problèmes humains que comporte l'enseignement. C'est pourquoi son autre vision des tuteurs d'apprentissage prend une importance particulière. On peut en effet, considérer l'ordinateur dans un rôle de ressource parallèle d'investigation. Cette nouvelle approche permettra vraisemblablement de considérer l'enseignement comme un processus de prise de décisions comme tout autre système de gestion générale. 


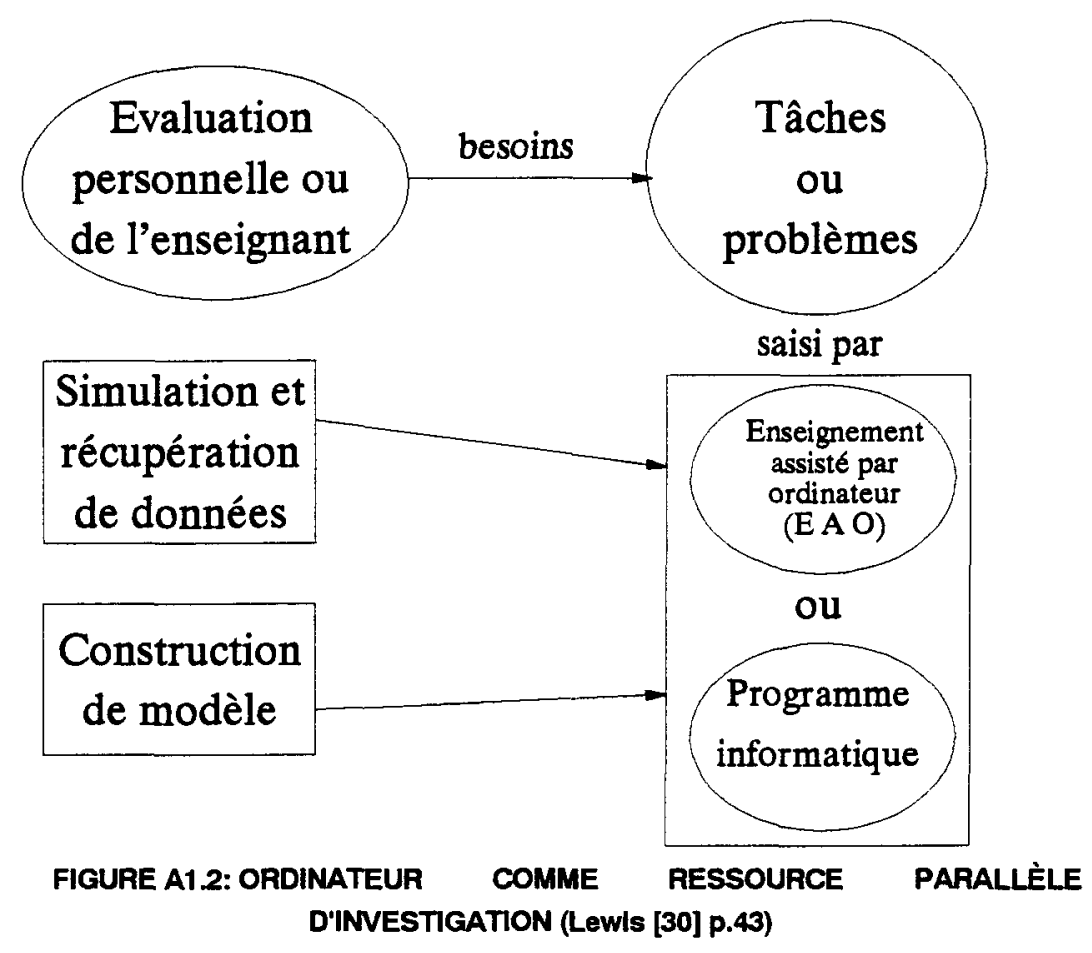

Remarquons que cet autre approche n'est fonctionnelle qu'en présence d'un enseignant. Elle lui apporte cependant une aide très précieuse en lui fournissant les données et problèmes dont il a besoin pour nourrir son enseignement. Toutefois, nous croyons que la situation deviendrait encore plus intéressante si on réunissait les deux approches. Ceci permettrait le remplacement temporaire de l'enseignant dans les situations où c'est souhaitable, et ce en utilisant l'ordinateur à deux niveaux. 


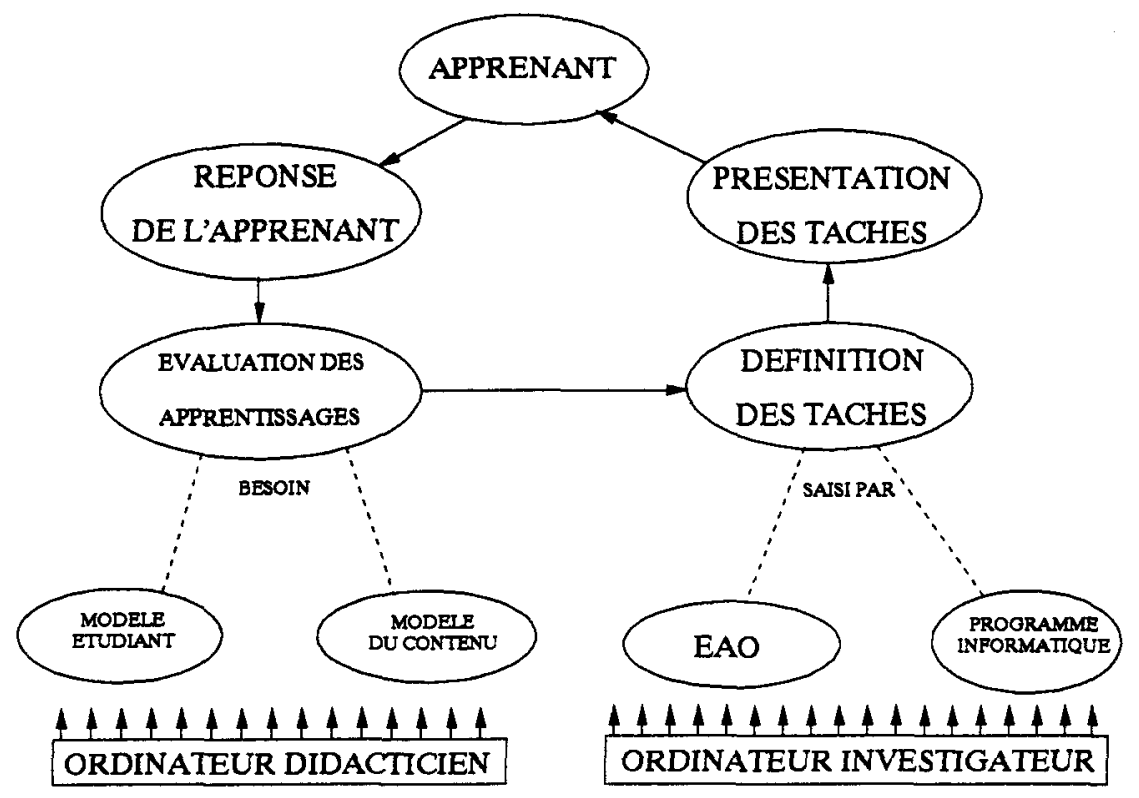

FIGURE A1.3: SYSTÈME TUTEUR À DEUX NIVEAUX POUR LA GESTION D'UNE SITUATION D'APPRENTISSAGE.

Cette concaténation des deux rôles de l'ordinateur permet d'obtenir un système complet en soi. Ce dernier possèdera l'avantage de pouvoir remplacer l'enseignant didacticien plus efficacement que le premier rôle selon Lewis puisqu'il pourra varier les stratégies d'apprentissage à l'aide du deuxième rôle. En effet, la partie investigation n'est pas limitée à la recherche de données. II sera également possible de l'utiliser à plusieurs niveaux tels:

- la recherche d'activités d'apprentissage

- la recherche de modèles de présentation

- l'évaluation des apprentissages

- l'évaluation des pertinences des contenus

- l'évaluation du profil psychologique de l'apprenant

- etc.

En définissant un nouveau tuteur qui possède les deux rôles simultanément, nous enrichissons considérablement notre vision des tuteurs. Nous pouvons de ce fait songer à des systèmes beaucoup plus intéressants pour l'apprenant et l'enseignant. 


\section{ANNEXE 2}

\section{SYSTEMES A TROIS BASES DE CONNAISSANCES}




\section{ANNEXE 2 \\ SYSTEMES A TROIS BASES DE CONNAISSANCES}

II peut arriver dans certains cas que l'une des bases soit beaucoup moins importante stratégiquement que les autres pour quelque raison que ce soit. Par exemple, dans le cas d'un robot, semblable au bras manipulateur, la base contexte n'a plus aucune importance puisque pour décider quels mouvements le bras devra effectuer (l'activité) il suffit de connaître la position actuelle relative des objets (le résultat) et la position relative désirée (le programme). Ce qui implique l'inutilité de la base Contexte et cette base sera alors dégénérée.

\section{A2.1: SYSTÈME AVEC UNE BASE DÉGÉNÉRÉE}

Dans le cas où une des bases est complètement inutile, il existe une manière relativement simple de s'en sortir sans modifier le système au complet. II s'agit de dégénérer une des bases (la base Contexte en ce qui concerne le bras manipulateur) en la laissant à l'intérieur du système mais en annulant son importance. Les fonctions de transfert deviennent alors:

$$
\begin{aligned}
& \left|a_{i}\right|=\alpha_{i}\left(p_{k}, C_{i}\right)=\frac{\sqrt{\left|a_{i} p_{k}\right|^{2}+0\left|a_{i} c_{1}\right|^{2}+\mid \text { ai préc }\left.\right|^{2}}}{\sqrt{2}} \\
& \left|a_{i}\right|=\alpha_{i}\left(p_{k}, C_{1}\right)=\frac{\sqrt{\left|a_{i} p_{k}\right|^{2}+\left|a_{i} p r e ́ c\right|}}{\sqrt{2}}
\end{aligned}
$$




$$
\begin{aligned}
& \left|r_{i}\right|=\beta_{i}\left(c_{k}, a\right)=\frac{\sqrt{0\left|r_{i} c_{k}\right|^{2}+\left|r_{i} a\right|^{2}+\mid r_{i} \text { préc }\left.\right|^{2}}}{\sqrt{2}} \\
& \left|r_{i}\right|=\beta_{i}\left(c_{k}, a\right)=\frac{\sqrt{\left|r_{i} a\right|^{2}+\mid r_{i} \text { préc }\left.\right|^{2}}}{\sqrt{2}}
\end{aligned}
$$

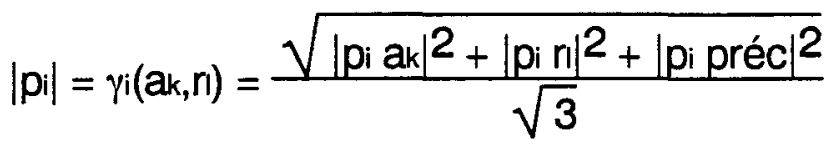

$$
\begin{aligned}
& \left|c_{i}\right|=\delta_{i}\left(r_{k}, p l\right)=\frac{\sqrt{0\left|c_{i} r_{k}\right|^{2}+0\left|c_{i} p_{1}\right|^{2}+\mid c_{i} \text { préc }\left.\right|^{2}}}{\sqrt{1}} \\
& \left|c_{i}\right|=\delta_{i}\left(r_{k}, p_{l}\right)=0
\end{aligned}
$$

Remarquons que cette approche, bien que fort pratique, ne correspond pas à la situation idéale puisqu'en agissant ainsi, le choix des activités ne dépendent que du programme et celui du résultat que de l'activité concernée. C'est pourquoi, dans le cas où une base se trouve complètement dégénérée, l'utilisation d'un système à trois bases de connaissances semble avantageux. 


\section{A2.2: SYSTÈME À TROIS BASES DE CONNAISSANCES}

Un système à trois bases (sans base Contexte par exemple) se schématise comme suit.

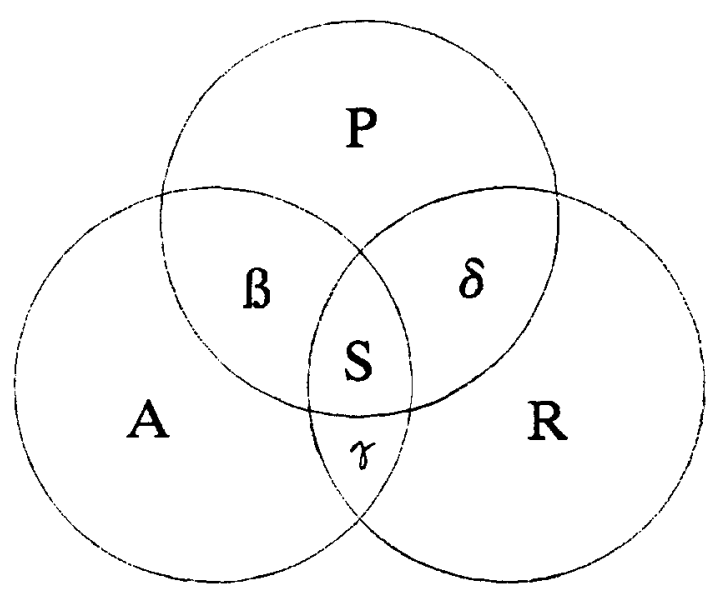

FIGURE A2.1: SYSTEME A TROIS BASES DE CONNAISSANCES

Dans ce cas, les fonctions de transfert deviennent:

$$
\begin{aligned}
& \left|\alpha_{i}\right|=\alpha\left(r_{k}, p_{i}\right)=\frac{\sqrt{\left|a_{i} r_{k}\right|^{2}+\left|a_{i} p_{1}\right|^{2}+\mid \text { ai préc }\left.\right|^{2}}}{\sqrt{3}} \\
& \left|\beta_{i}\right|=\beta\left(p_{k}, a l\right)=\frac{\sqrt{\left|r_{i} p_{k}\right|^{2}+\left|r_{i} a\right|^{2}+\left|r_{i} p r e ́ c\right|}}{\sqrt{3}} \\
& \left|\gamma_{i}\right|=\gamma(a k, n)=\frac{\sqrt{\left|p_{i} a_{k}\right|^{2}+\left|p_{i} n_{1}\right|^{2}+\mid p_{i} \text { préc }\left.\right|^{2}}}{\sqrt{3}}
\end{aligned}
$$

L'avantage de cette approche par rapport à celle où une base est dégénérée est qu'à chaque étape, la décision est prise en considérant les résultats des deux 
autres bases. Par contre, cette approche n'est souhaitable que dans le cas où une base a réellement une importance nulle, c'est pourquoi, dans la plupart des cas, un système à quatre bases sera utilisé. 


\title{
UNIVERSITE DU QUEBEC
}

\author{
RAPPORT TECHNIQUE \\ ACCOMPAGNANT \\ LE \\ MEMOIRE
}

\section{MODELISATION D'UN SYSTEME GENERAL DE GESTION INTELLIGEMMENT ASSISTE PAR ORDINATEUR (EIAO)}

Application à l'enseignement de l'algèbre de Boole et à la gestion d'un bras manipulateur(robotique)

\author{
PAR \\ LINDA GELEYN \\ B.SC.
}

MARS 1992 


\section{TABLE DES MATIERES}

PARTIE 1 LISTAGE DU PROGRAMME PROLIN

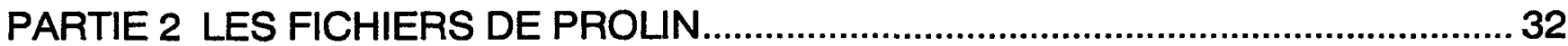

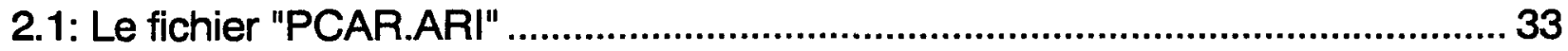

2.2: Le fichier "NORMES.ARI" .................................................................................. 37

PARTIE 3 LES CONTENUS DES BASES DE CONNAISSANCES.............................64

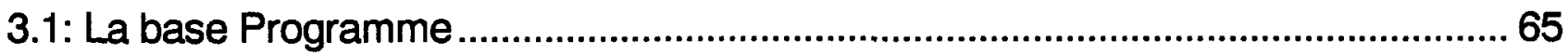

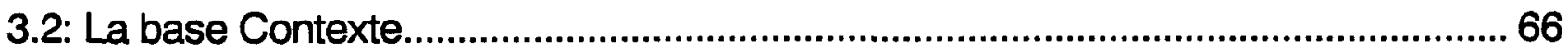

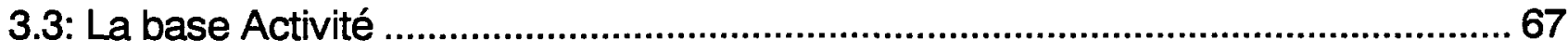

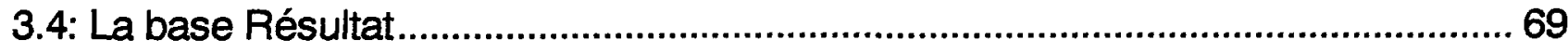

PARTIE 4 LE CAHIER D'ACTIVITES DE PROLIN...................................................... 70

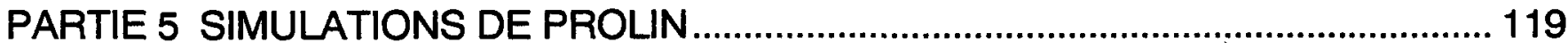

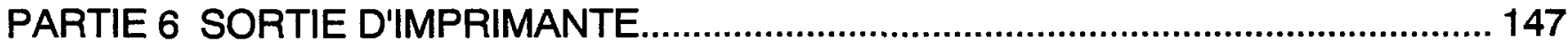




\section{PARTIE 1}

\section{LISTAGE DU PROGRAMME PROLIN}




\section{PARTIE 1}

\section{LISTAGE DU PROGRAMME PROLIN}

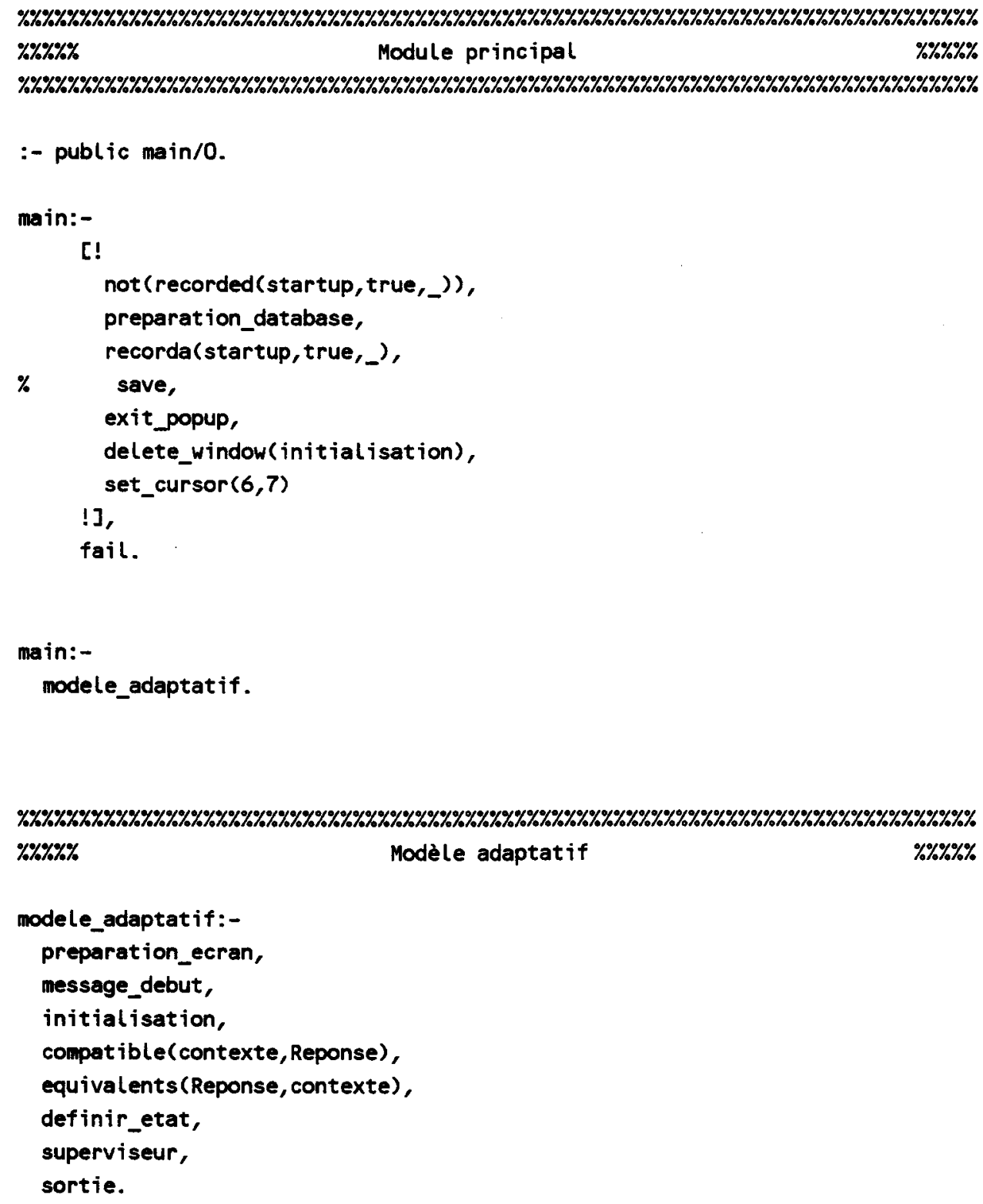


\%\%\%\%\%\%\%\%\%\%\%\%\%\%\%\%\%\%\%\%\%\%\%\%\%\%\%\%\%\%\%\%\%\%\%\%\%\%\%\%\%\%\%\%\%\%\%\%\%\%\%\%\%\%\%\%\%\%\%\%\%

$\% \% \%$

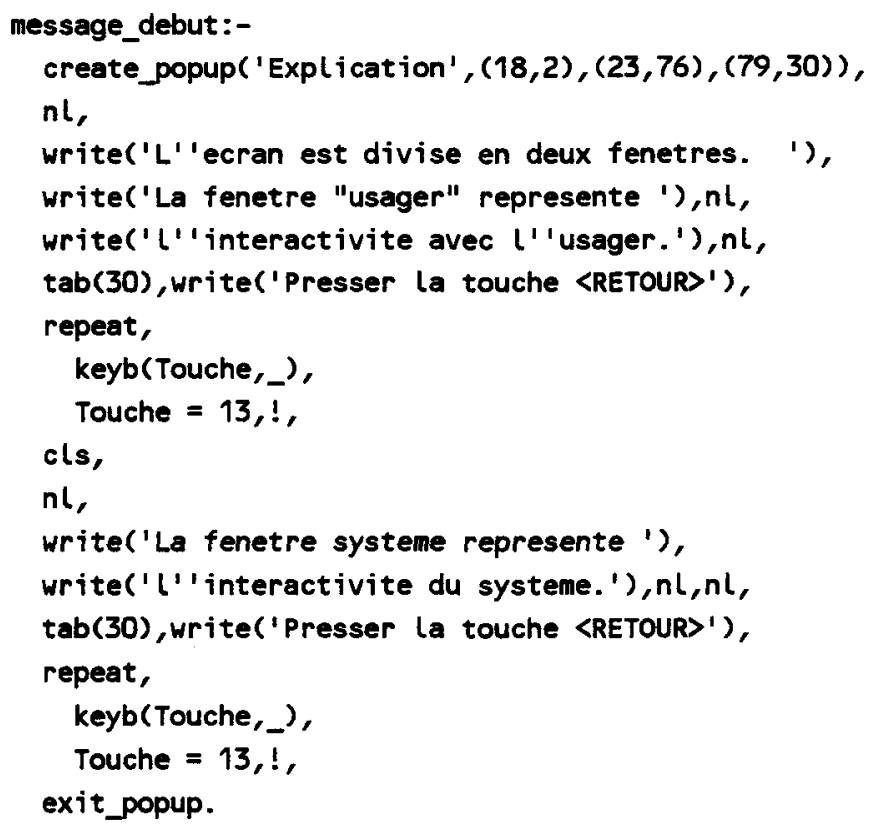




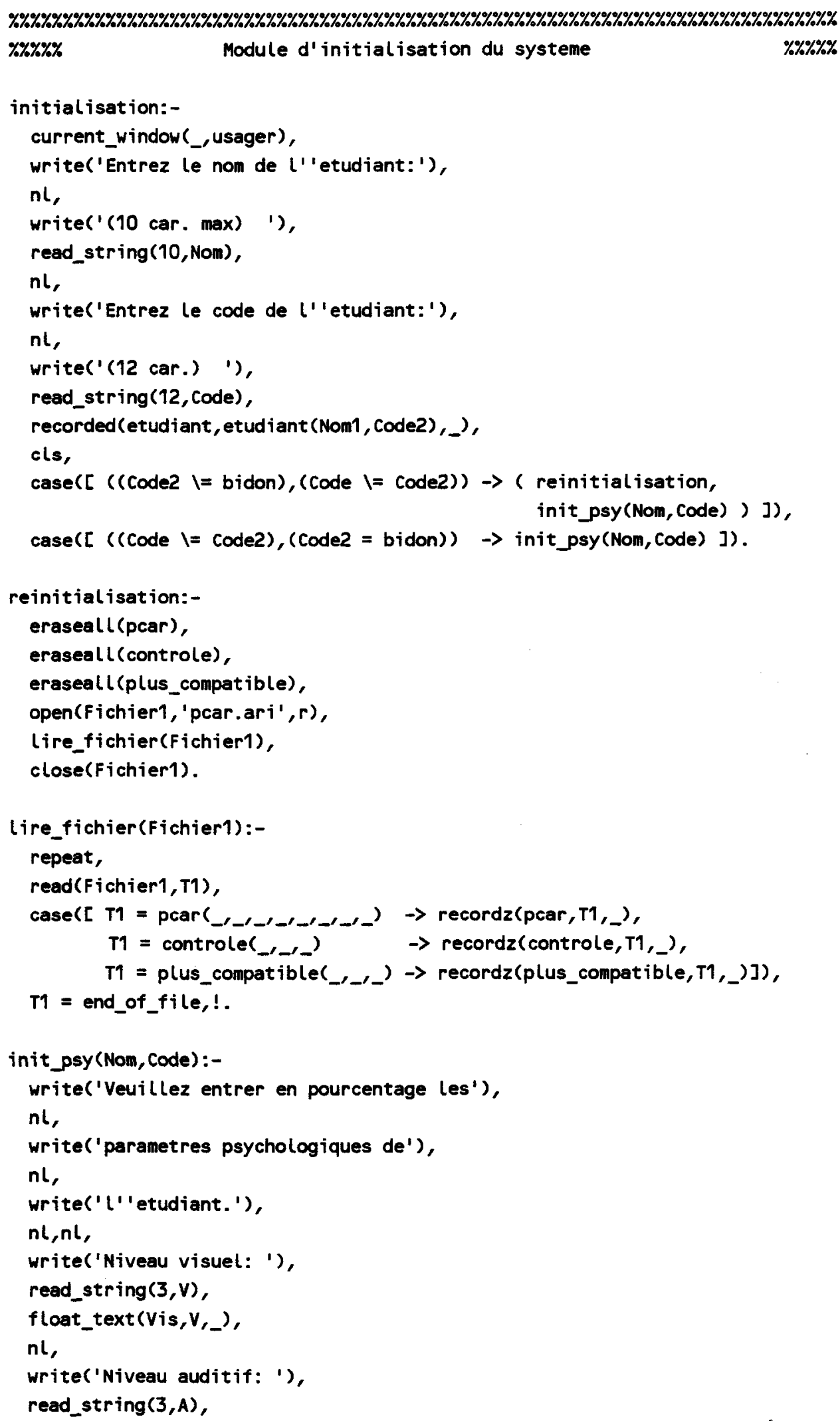




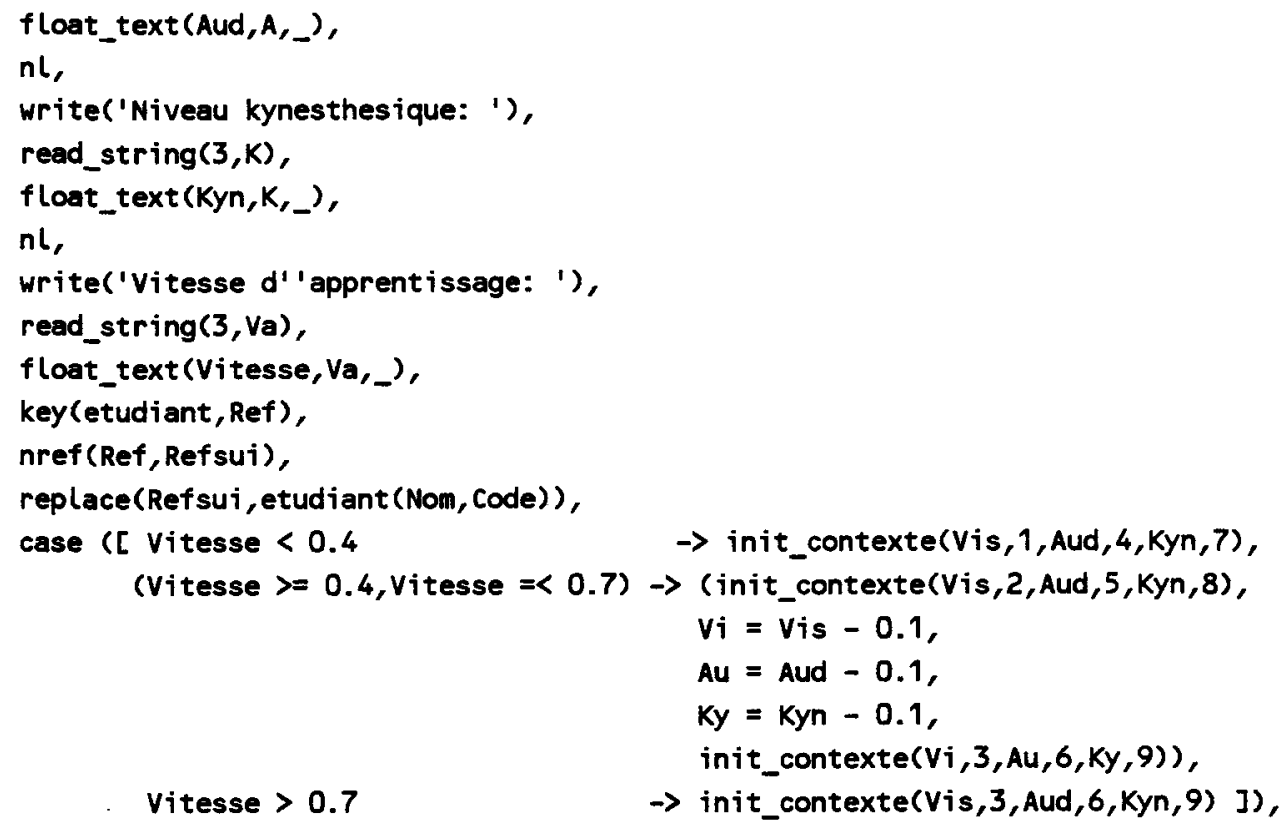




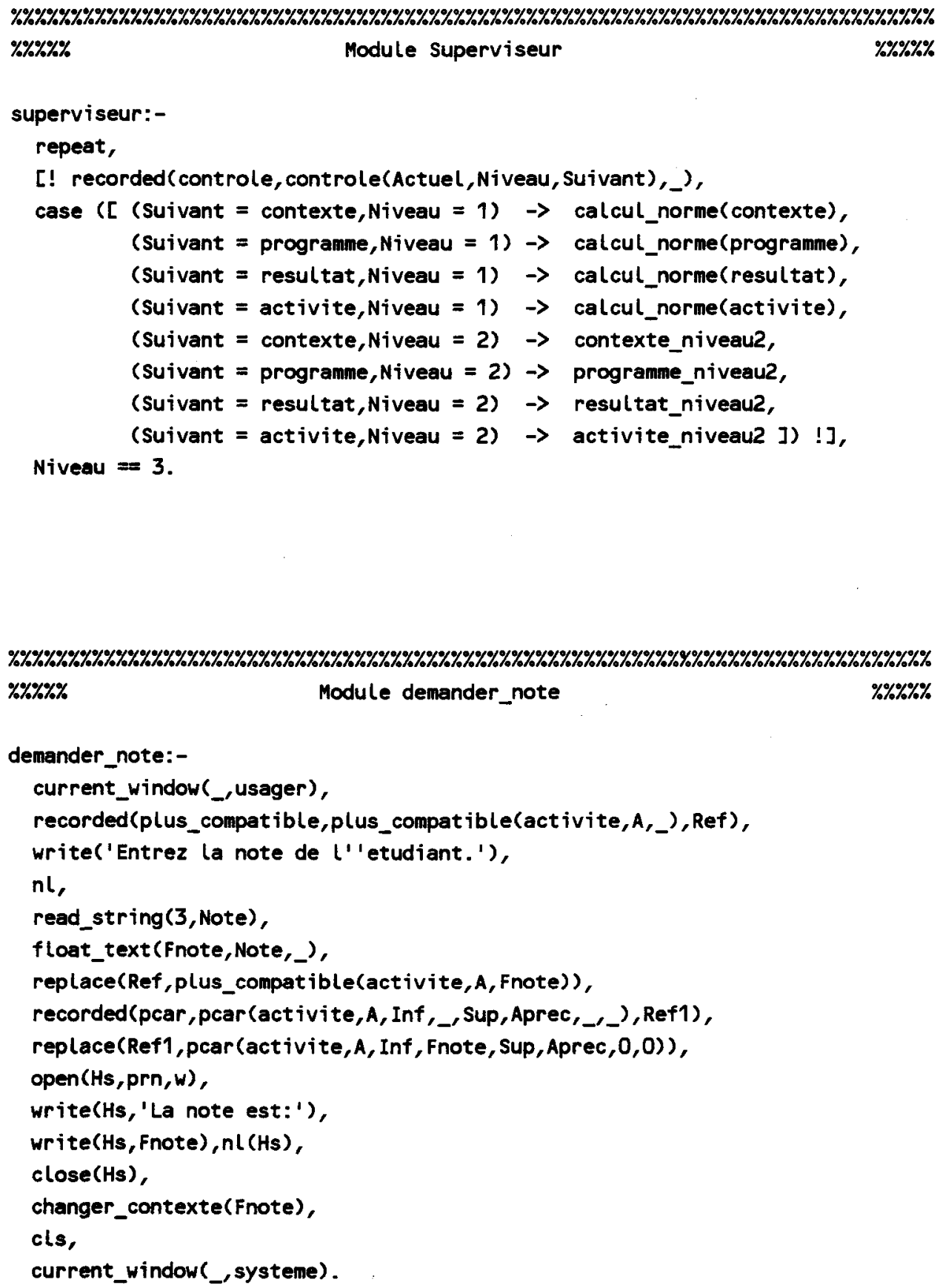

superviseur:-

repeat,

[! recorded (controle, controle(Actuel, Niveau, Suivant), ),

case $([$ (Suivant $=$ contexte, Niveau $=1) \rightarrow$ calcul_norme(contexte) ,

(Suivant = programme,Niveau = 1 ) $\rightarrow$ calcul_norme(programme),

(Suivant $=$ resultat,Niveau $=1$ ) $\rightarrow$ calcul_norme(resultat),

(Suivant = activite,Niveau $=1$ ) $\rightarrow$ calcul_norme(activite),

(Suivant $=$ contexte, Niveau $=2$ ) $\rightarrow$ contexte_niveau2,

(Suivant $=$ programme,Niveau $=2$ ) $\rightarrow$ programme_niveau2,

(Suivant $=$ resultat,Niveau $=2$ ) $\rightarrow$ resultat_niveau2,

(Suivant = activite,Niveau $=2$ ) $\rightarrow$ activite_niveau2 ]) !],

Niveau $=3$. 


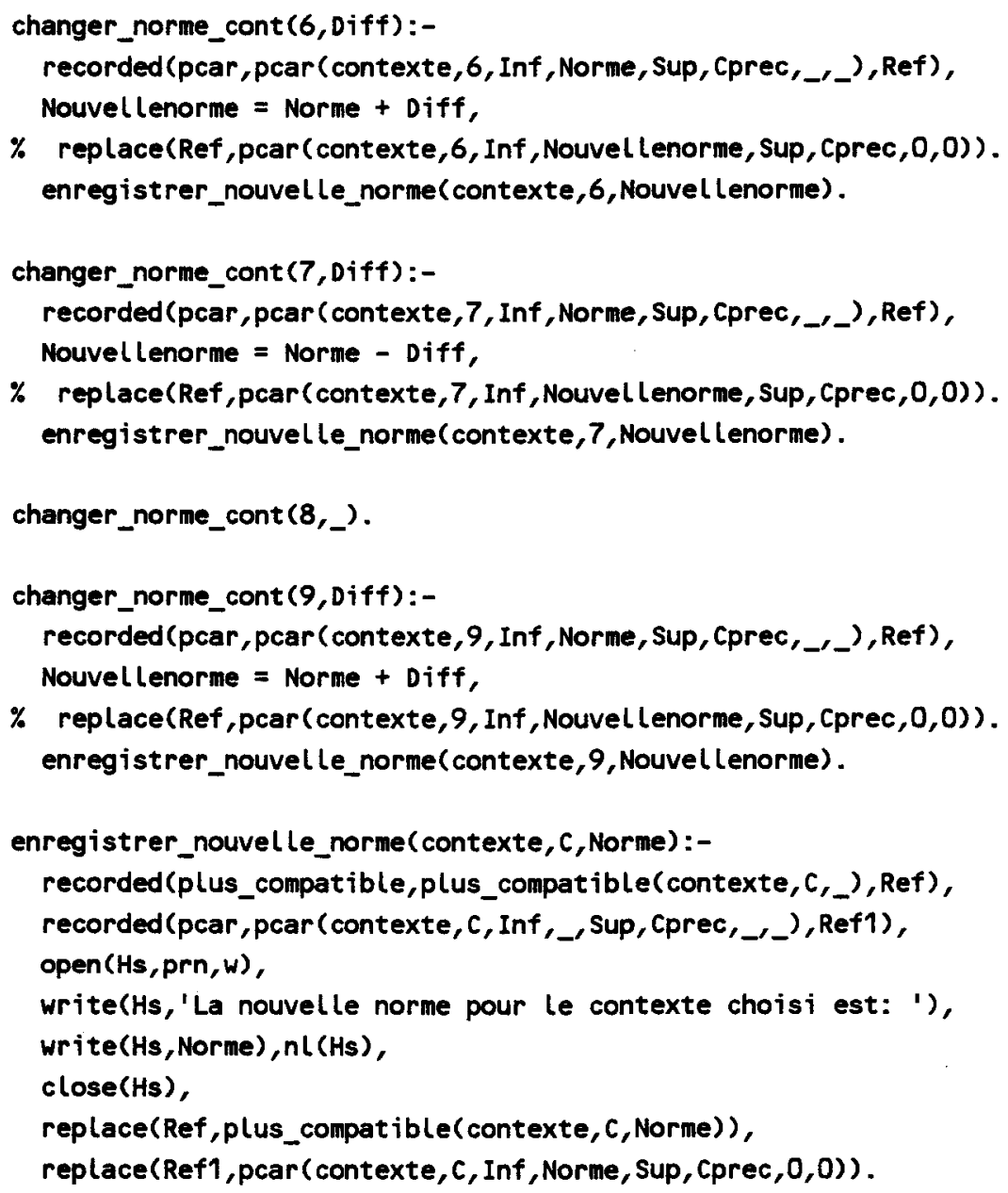




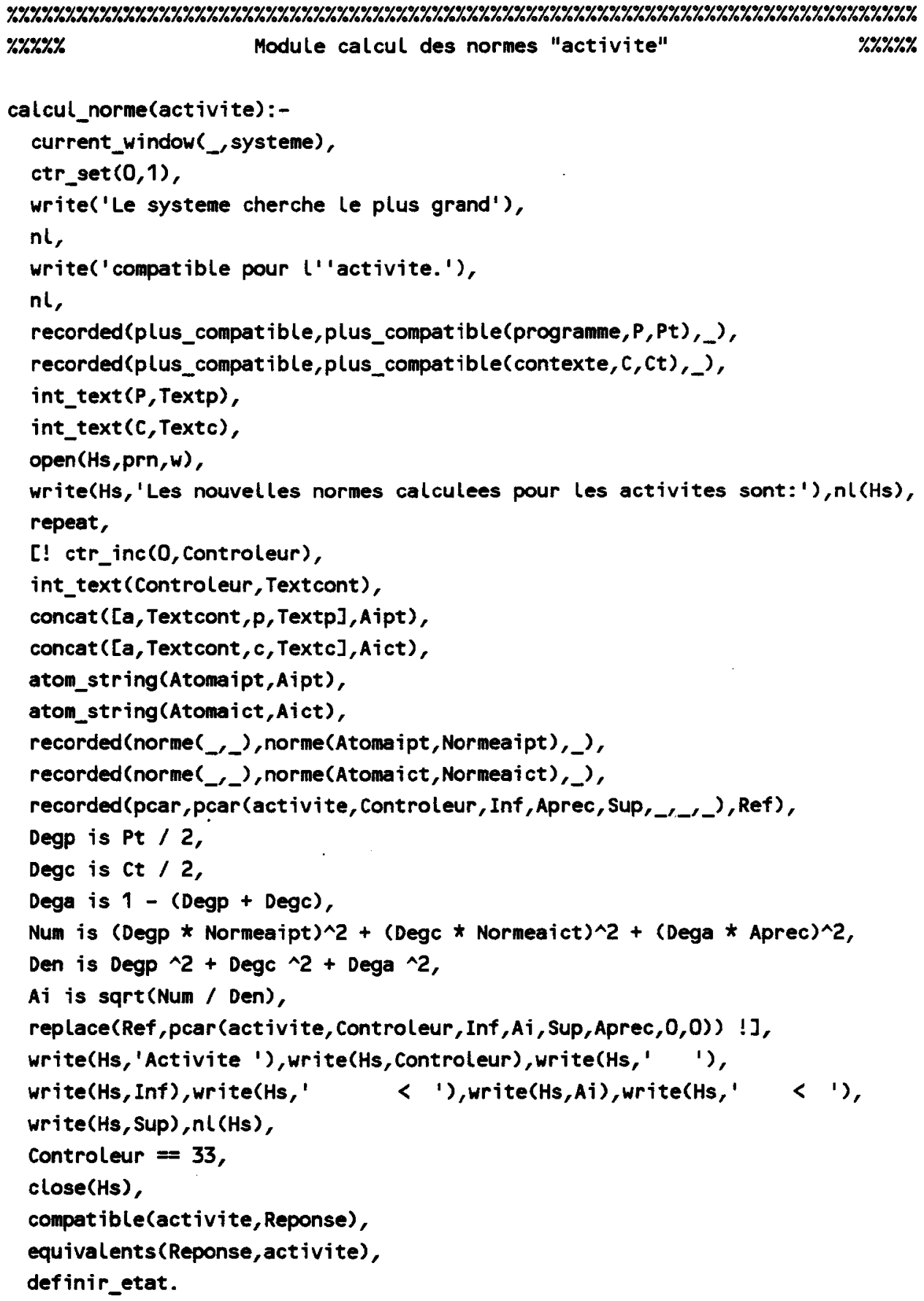

calcul_norme(activite):-

current_window(_,systeme), 


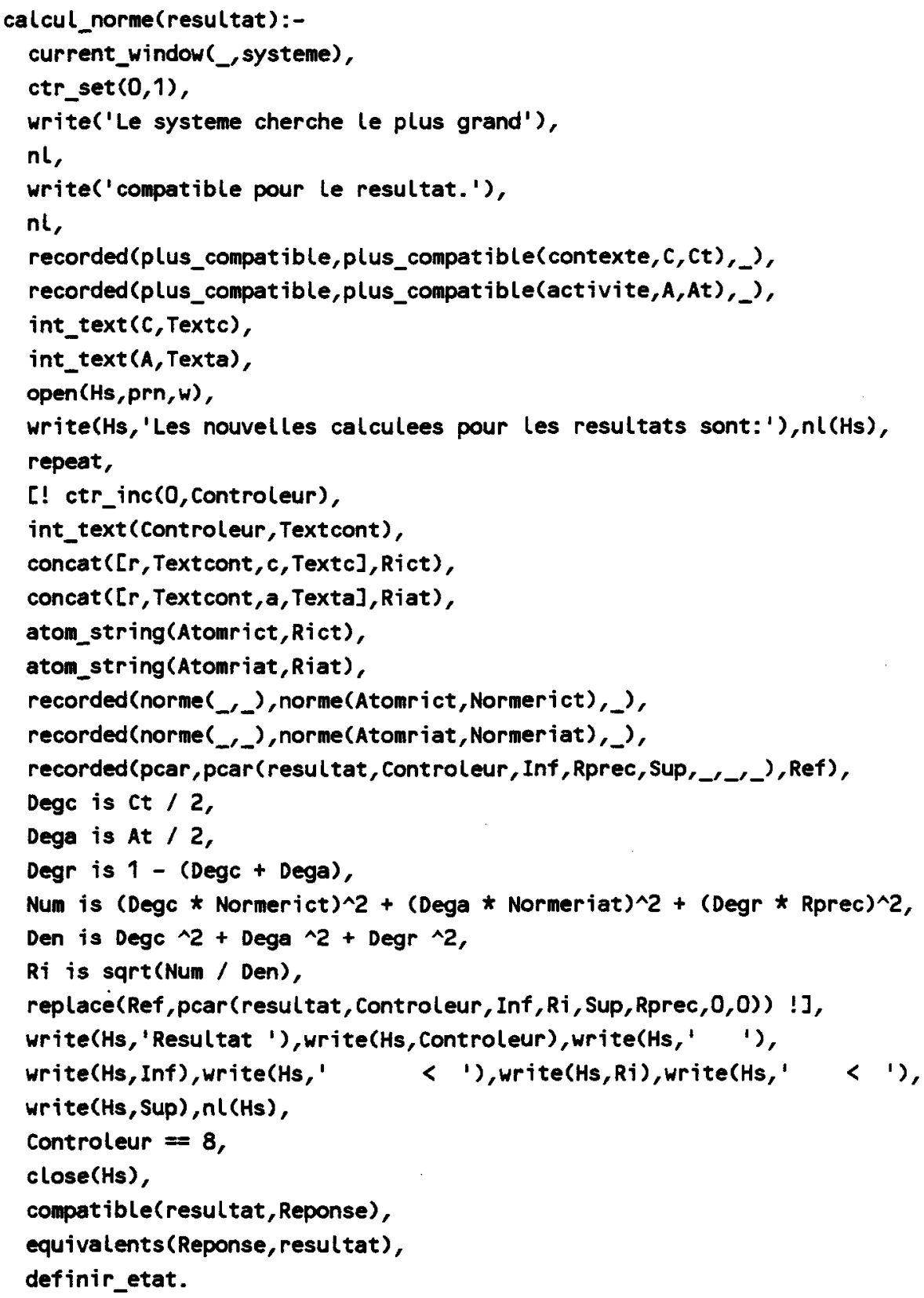




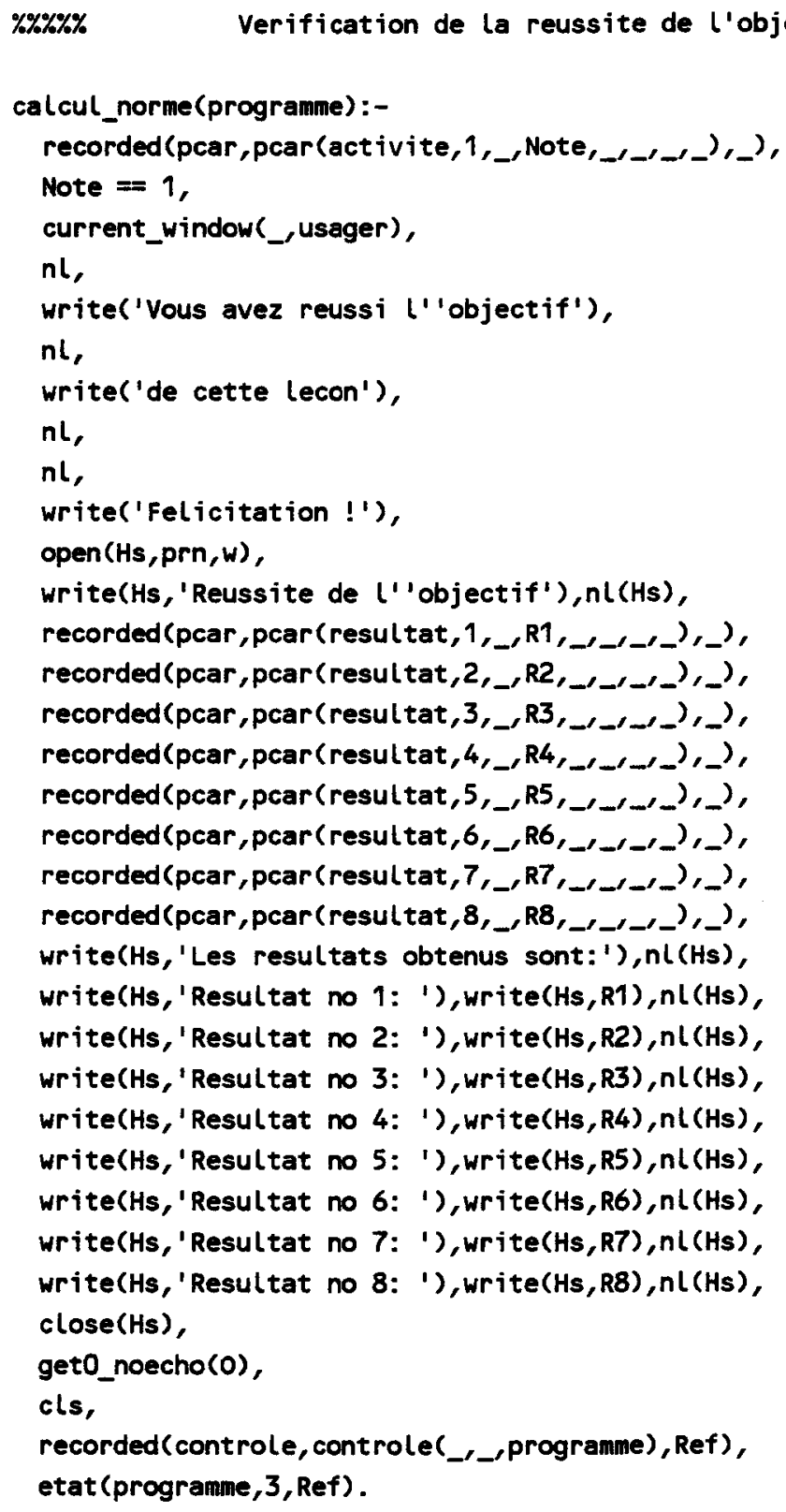




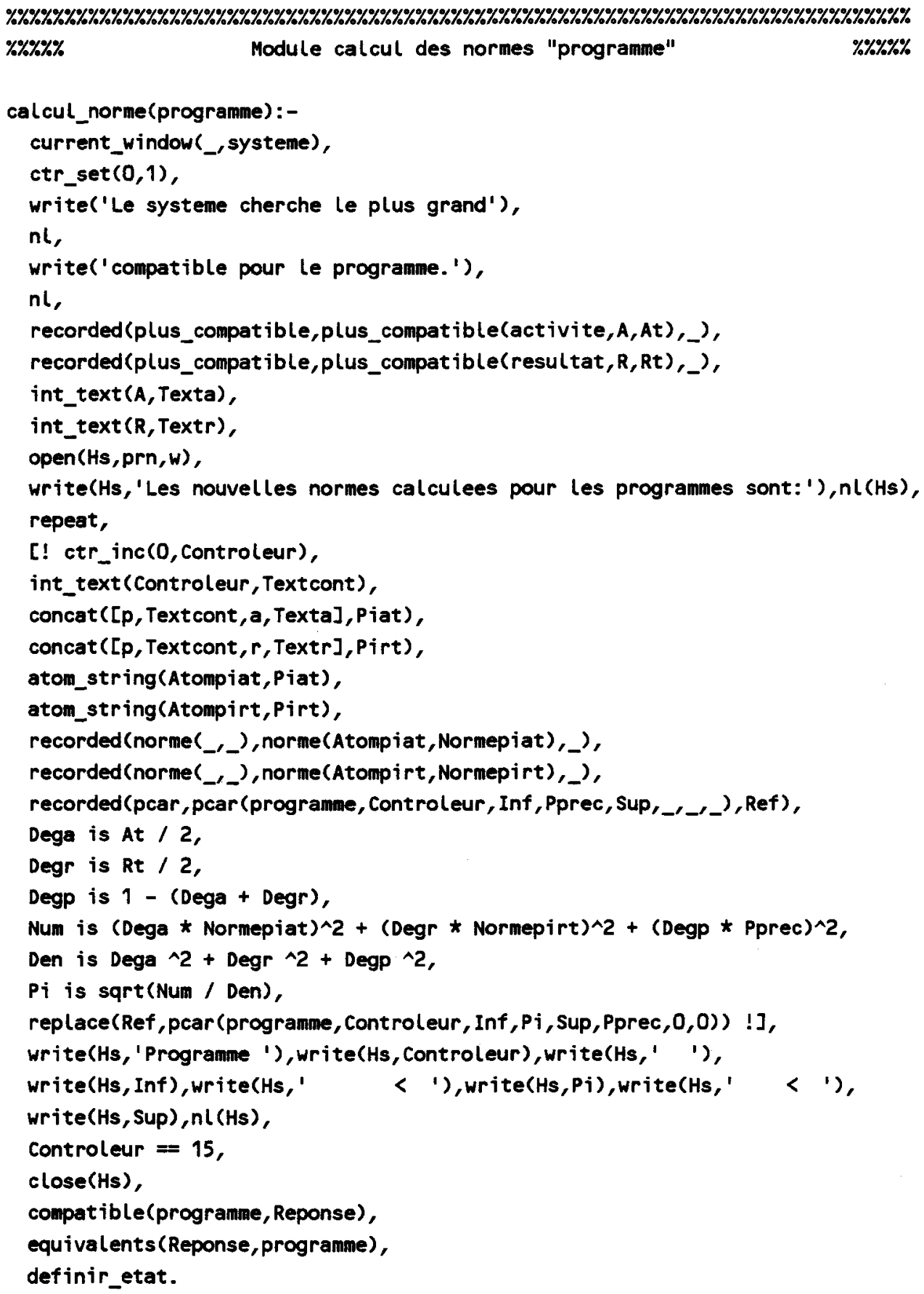




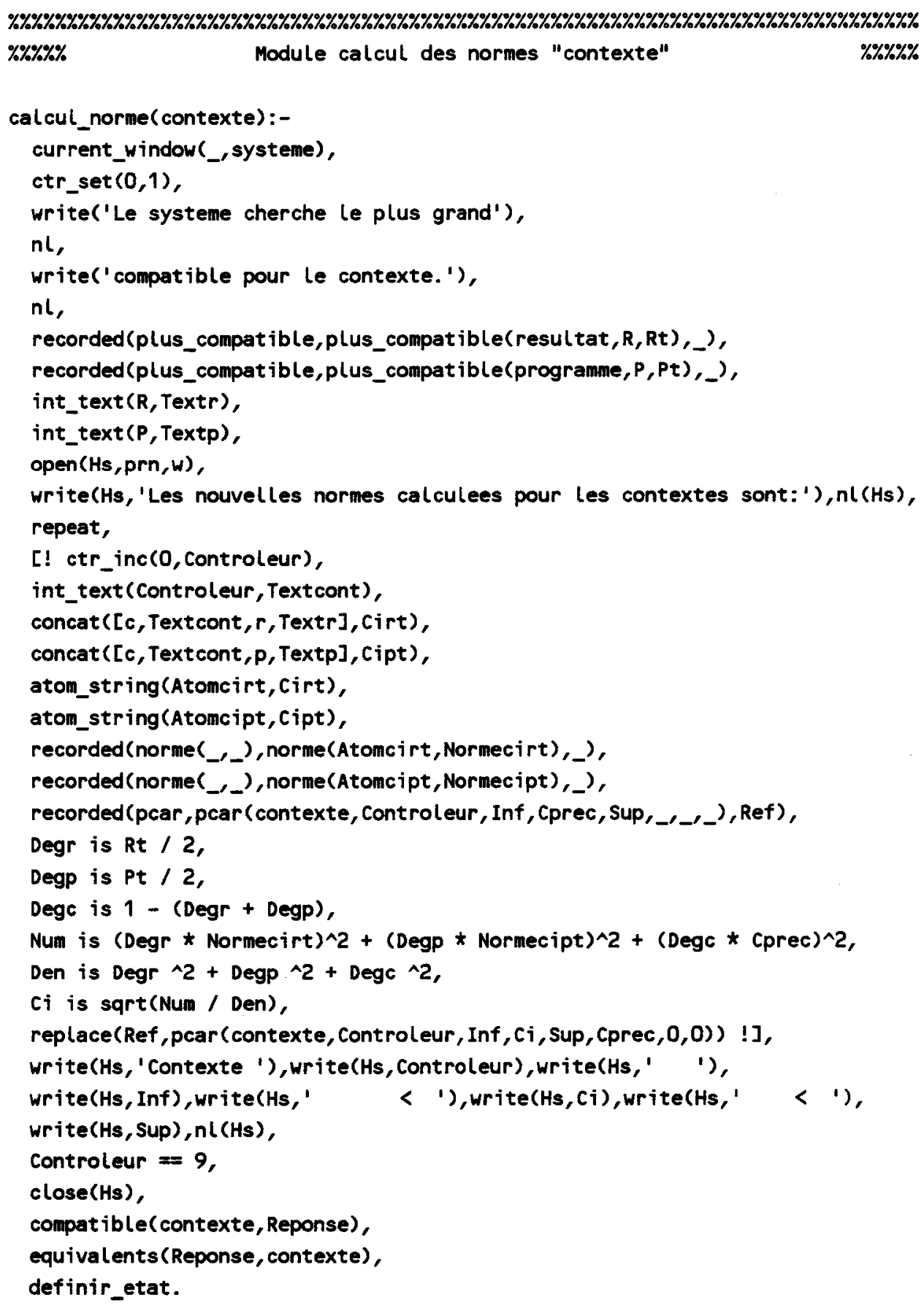




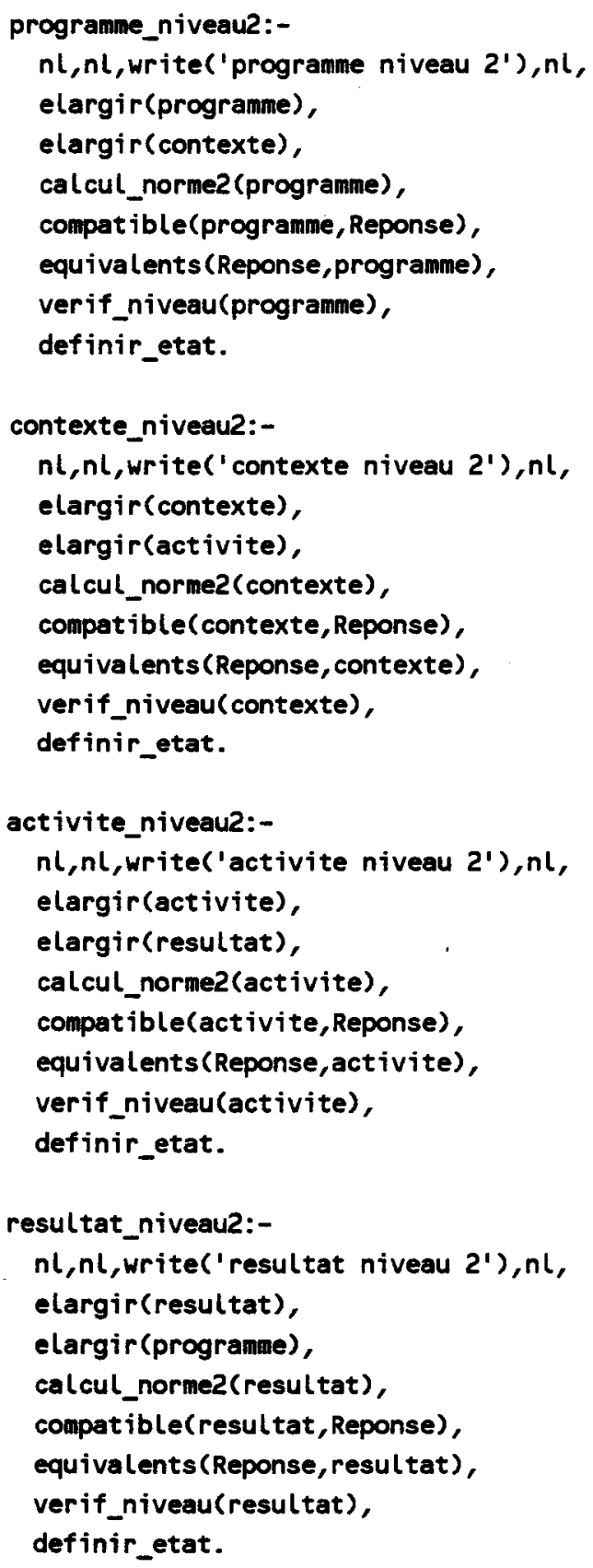




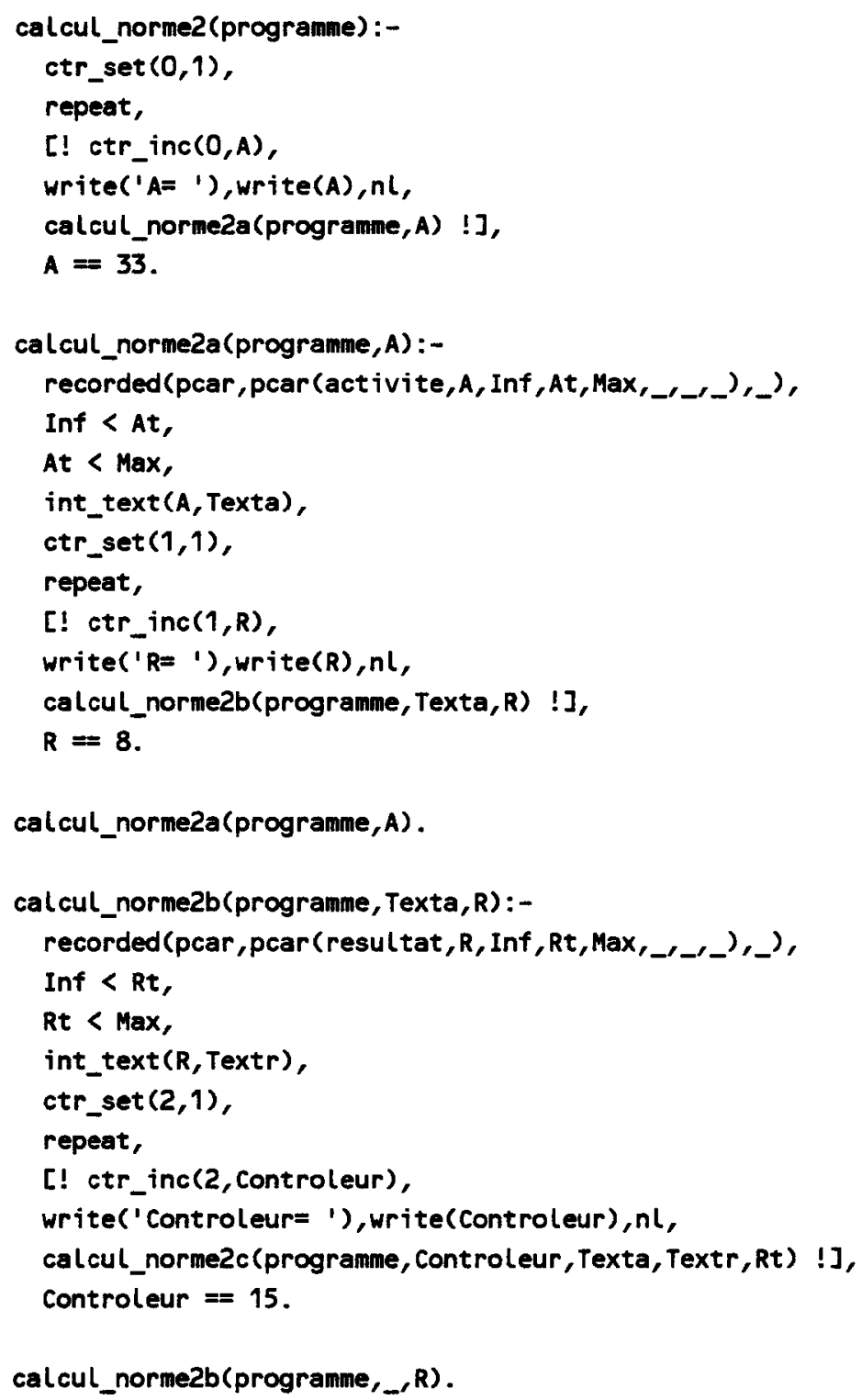




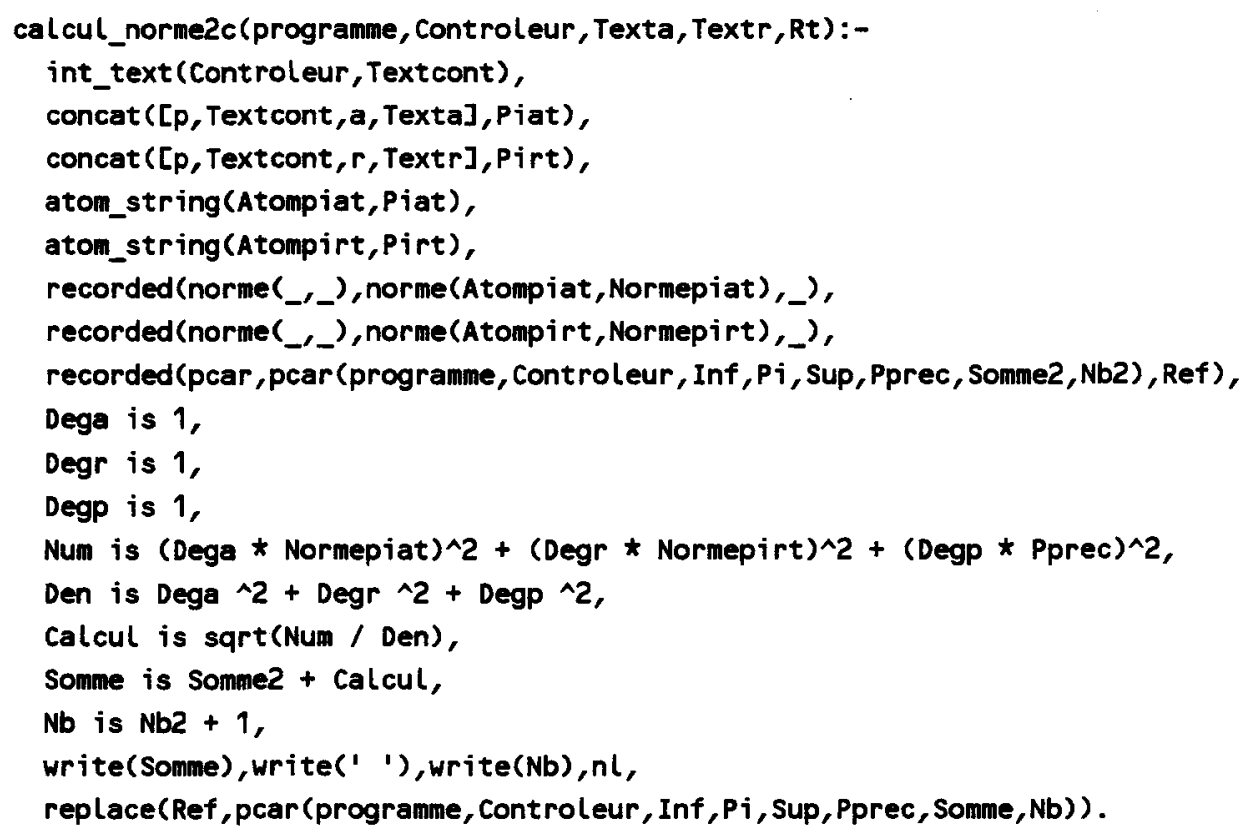




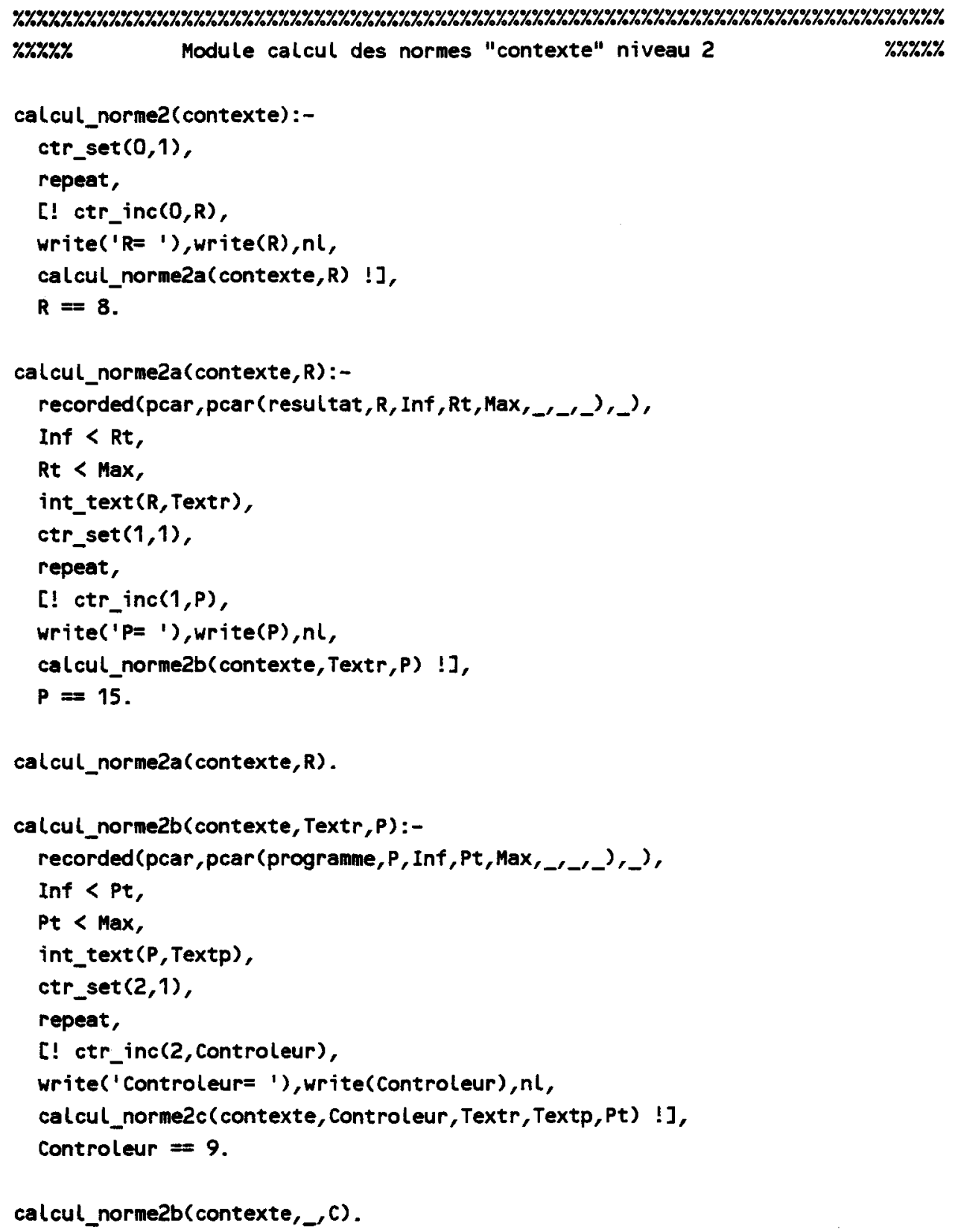




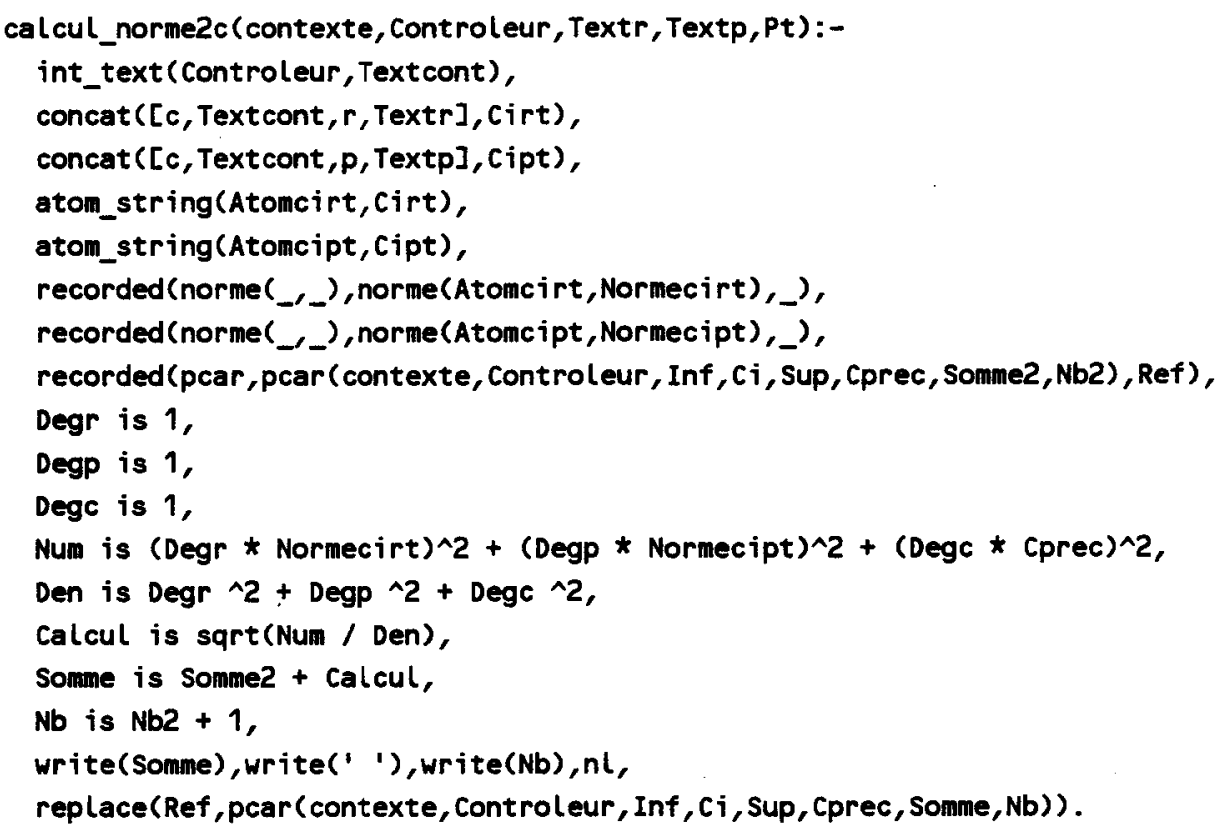




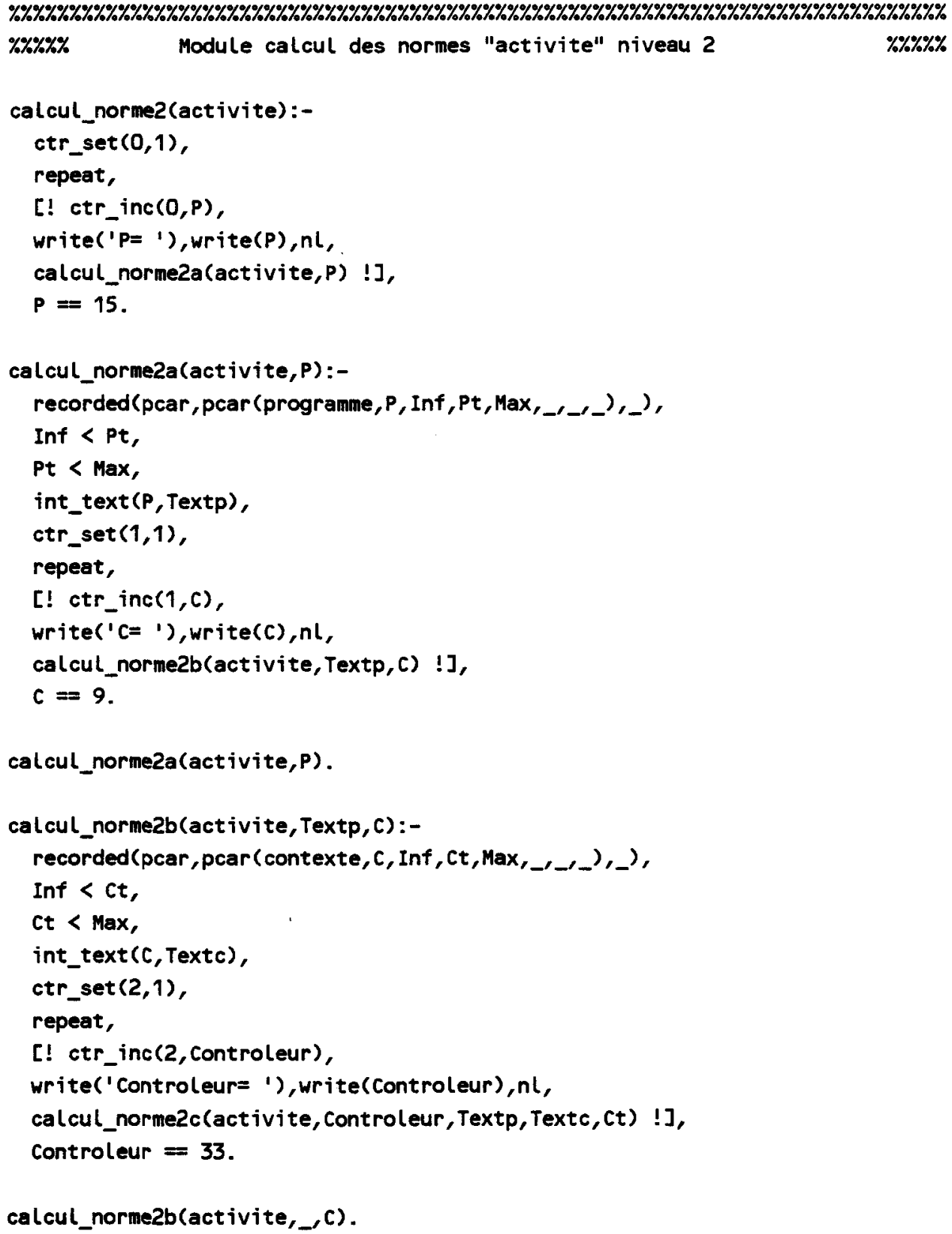




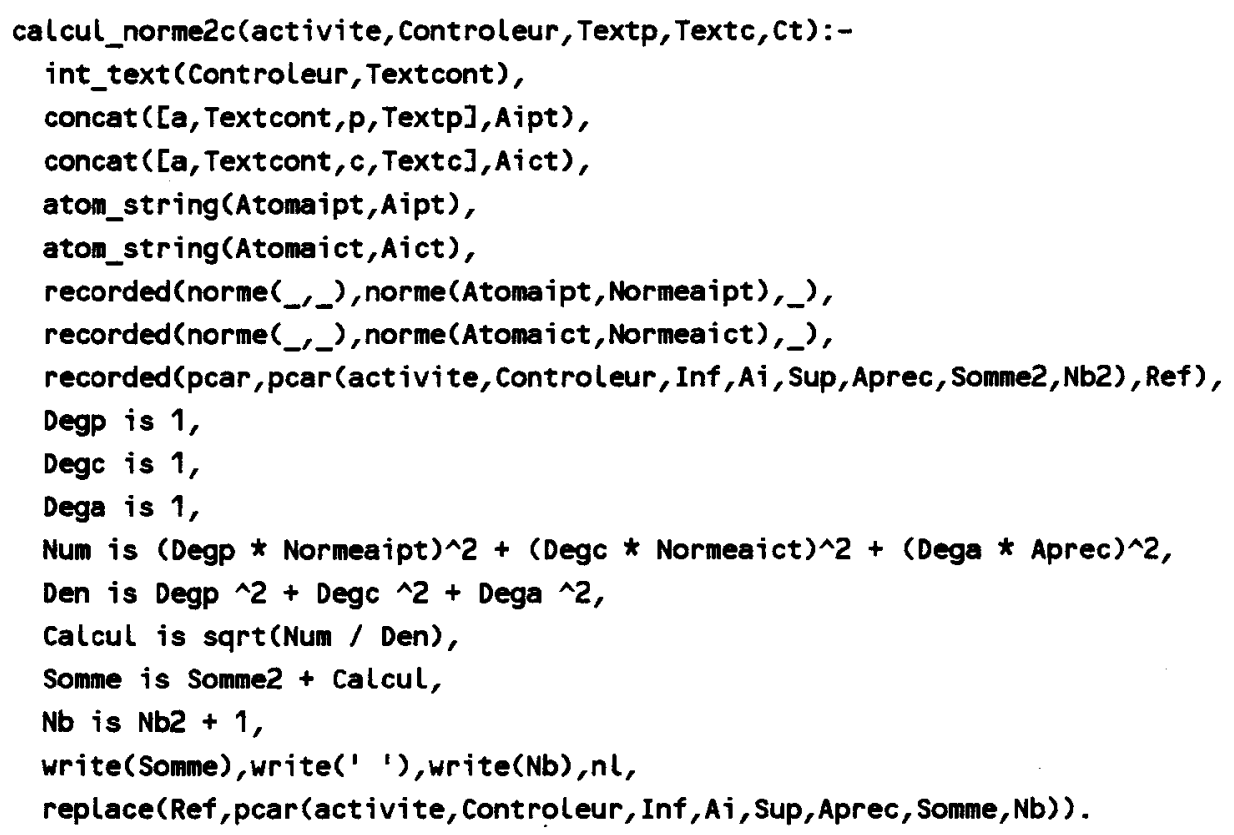




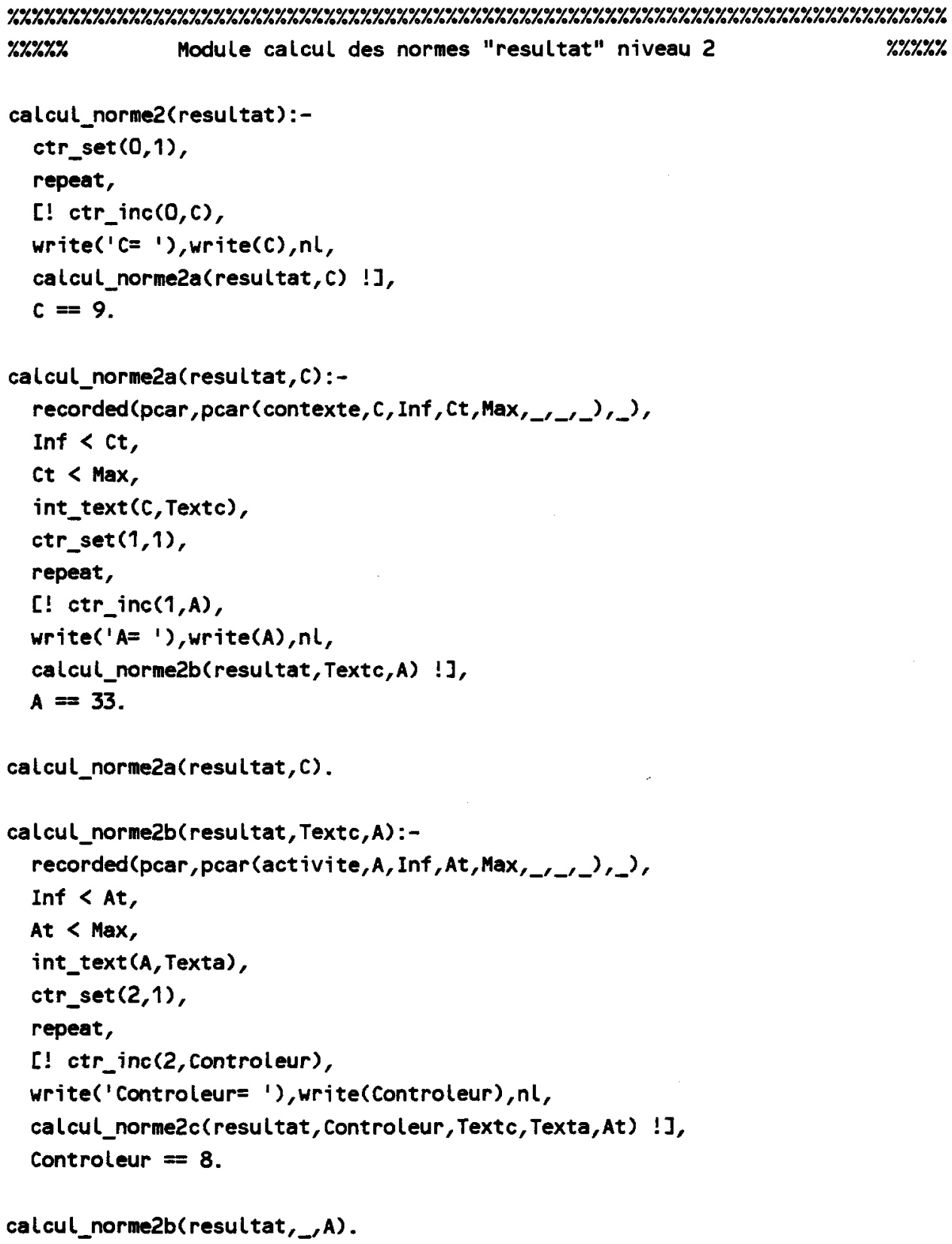




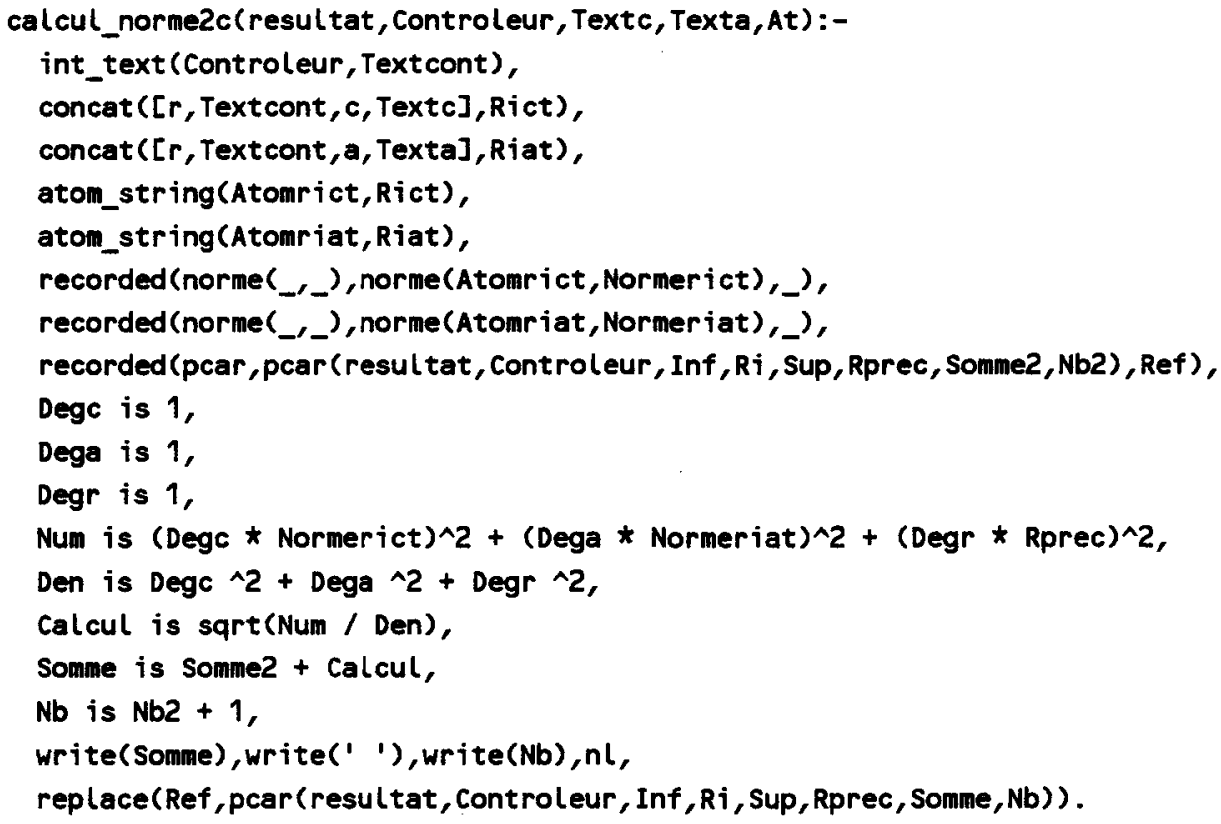




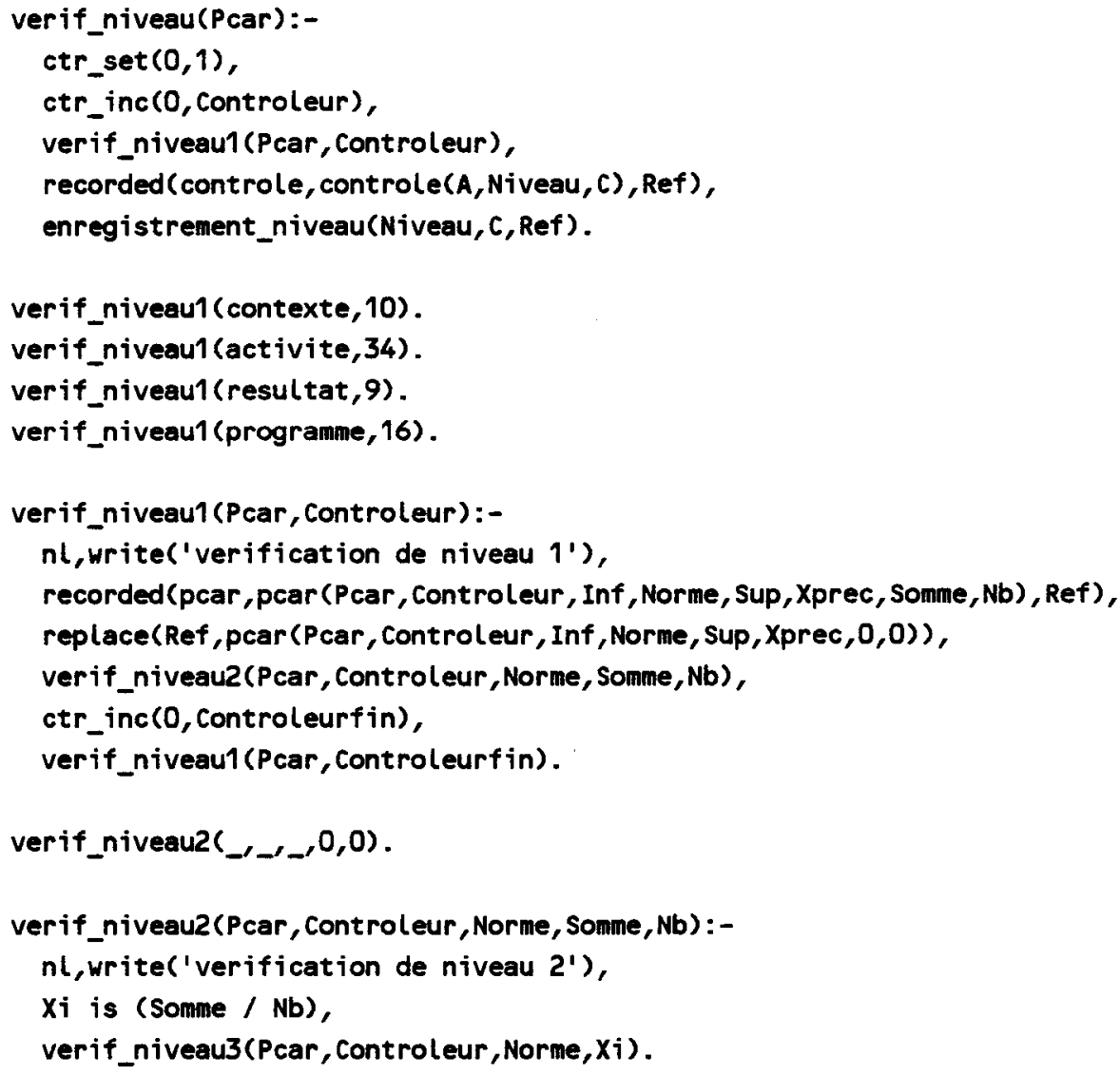

verif_niveau3 (Pcar, Controleur, Norme, Norme).

verif_niveau3(Pcar, Controleur, Norme, $X i$ ):nl, write ('verification de niveau 3 '), recorded (pcar, pcar (Pcar, Controleur, Inf,_, Sup, Xprec,_,_), Ref), replace(Ref, pcar (Pcar, Controleur, Inf, Xi, Sup, Xprec, 0, 0)), recorded (controle, controle $(A, B, C), \operatorname{Ref} 1)$, replace (Ref1, controle $(C, 1, C))$. 
\%\%\%\%\%\%\%\%\%\%\%\%\%\%\%\%\%\%\%\%\%\%\%\%\%\%\%\%\%\%\%\%\%\%\%\%\%\%\%\%\%\%\%\%\%\%\%\%\%\%\%\%\%\%\%\%\%\%\%\%\%

$\% \% \%$

enregistrement_niveau(1, $C$, Ref).

enregistrement_niveau(Niveau, $C$, Ref) :-

replace (Ref, controle $(C, 3, C))$. .

\%\%\%\%\%\%\%\%\%\%\%\%\%\%\%\%\%\%\%\%\%\%\%\%\%\%\%\%\%\%\%\%\%\%\%\%\%\%\%\%\%\%\%\%\%\%\%\%\%\%\%\%\%\%\%\%\%\%\%\%\%\%\%\%\%\%\%

$\% \% \%$

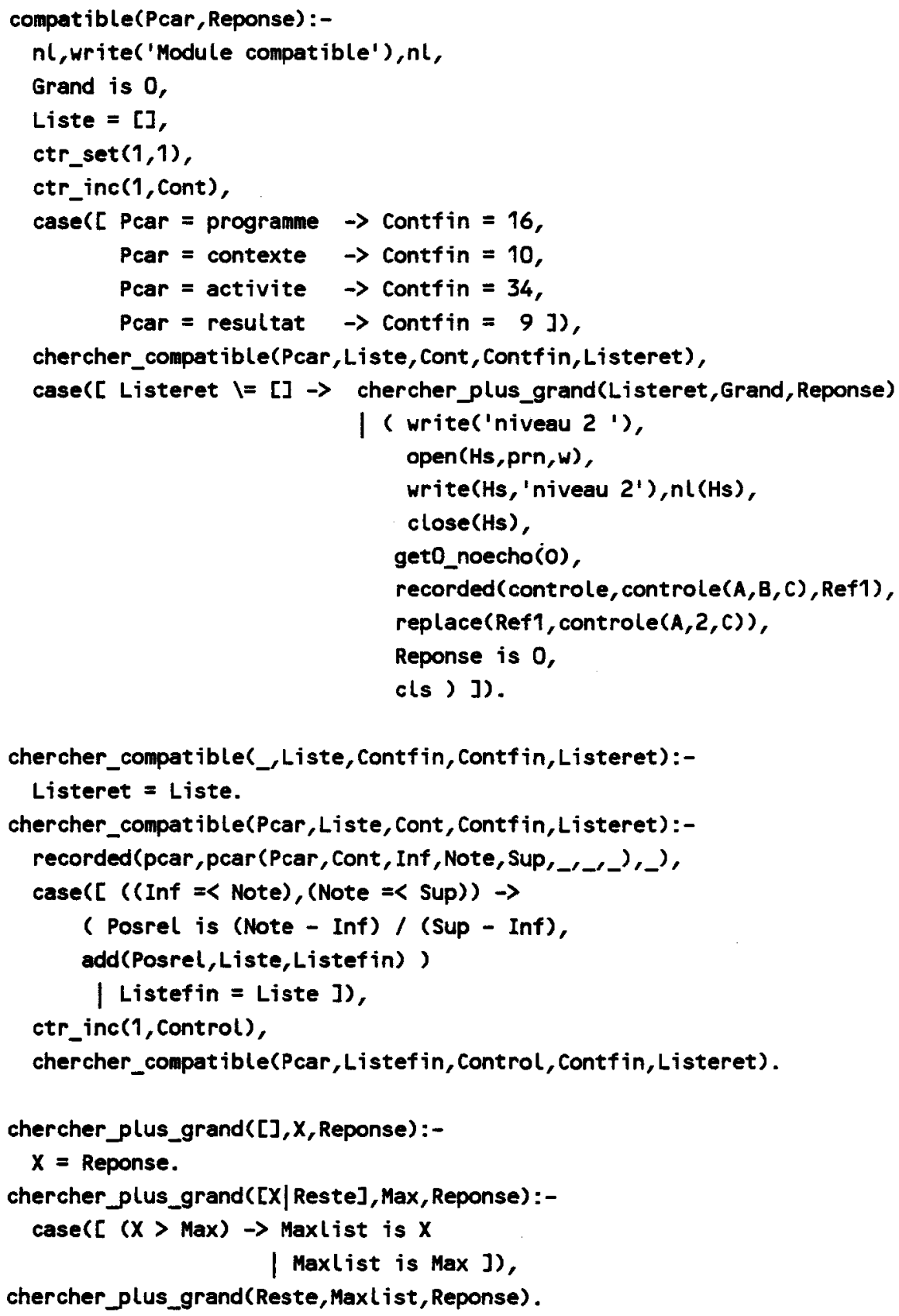




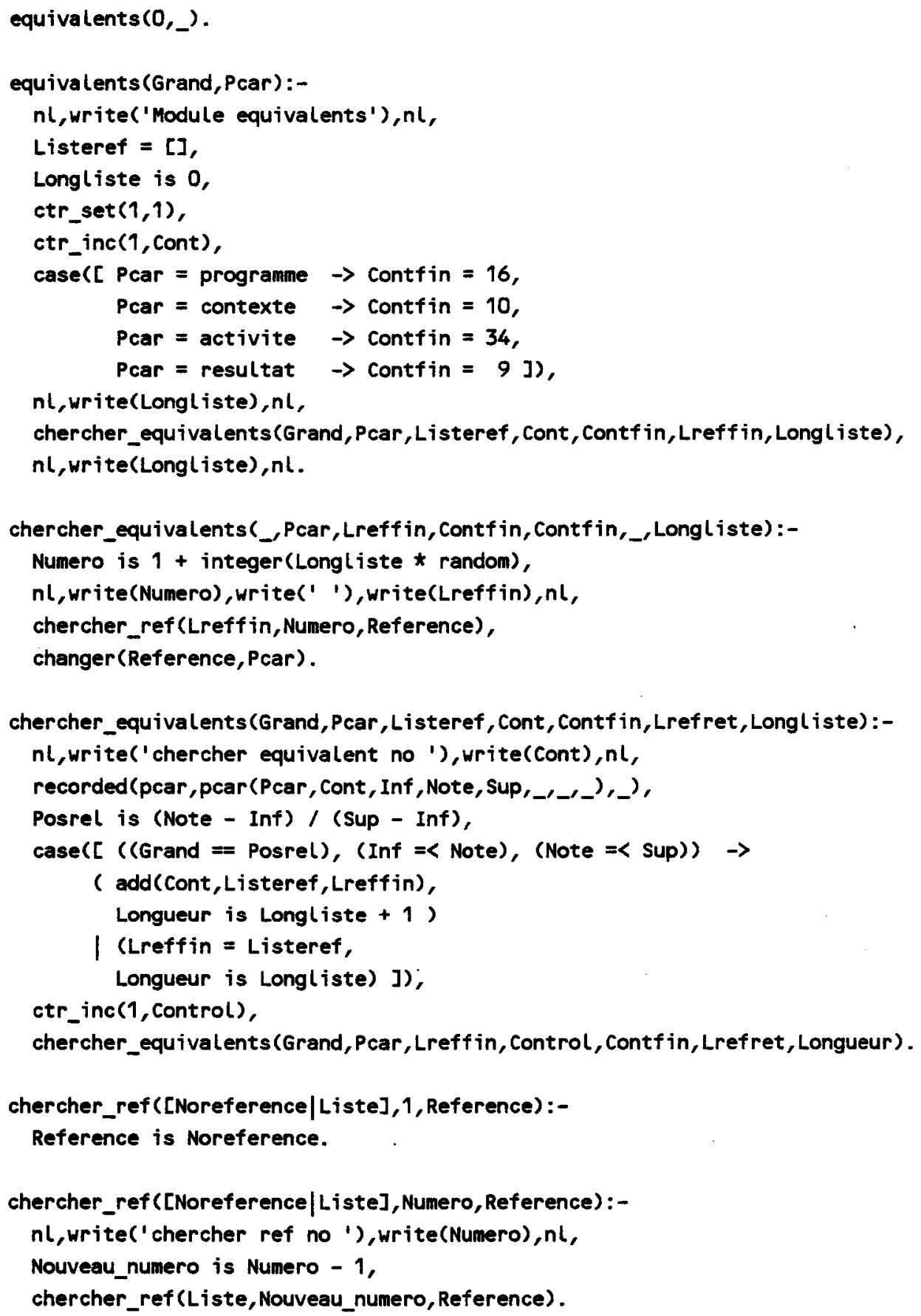




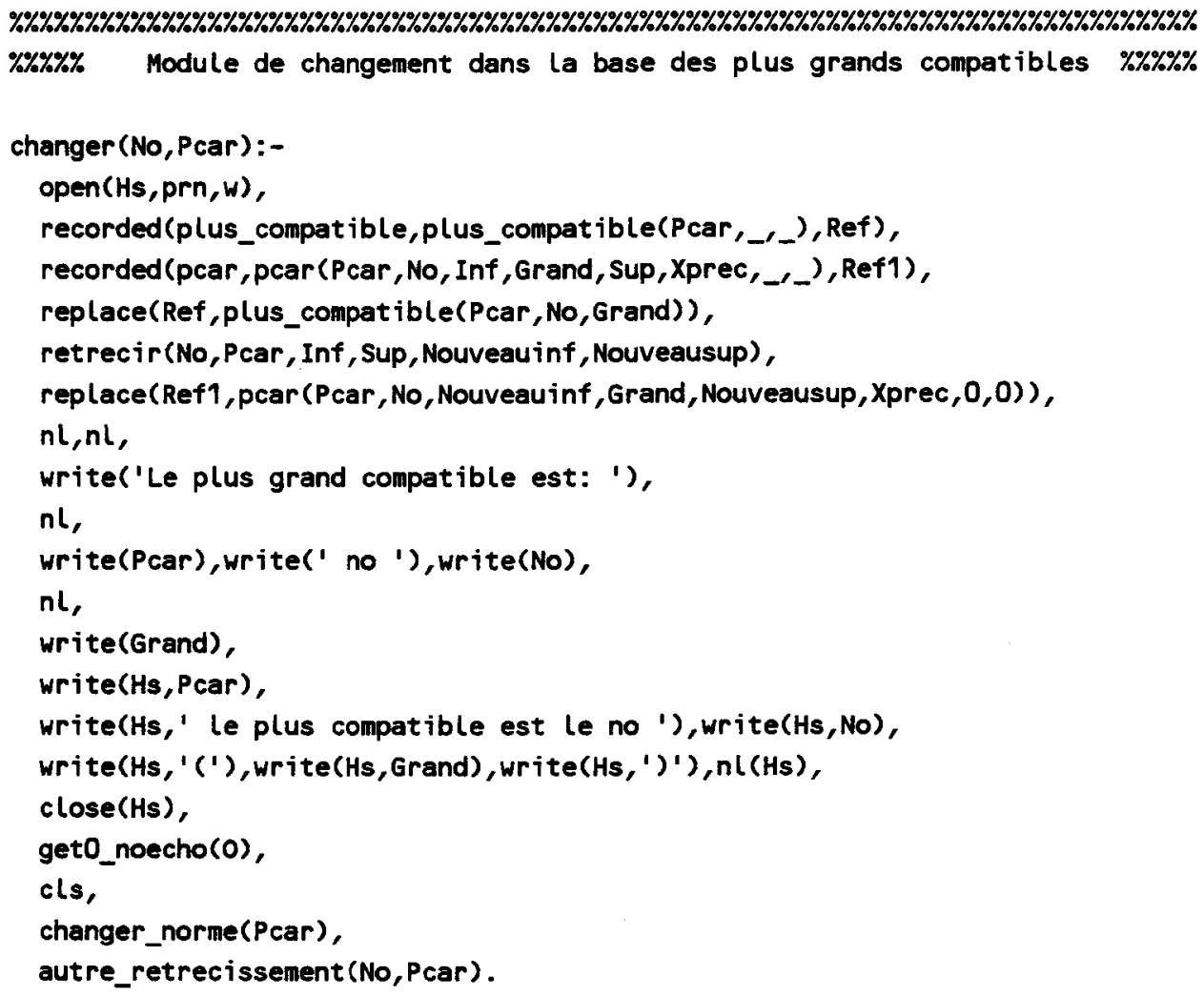




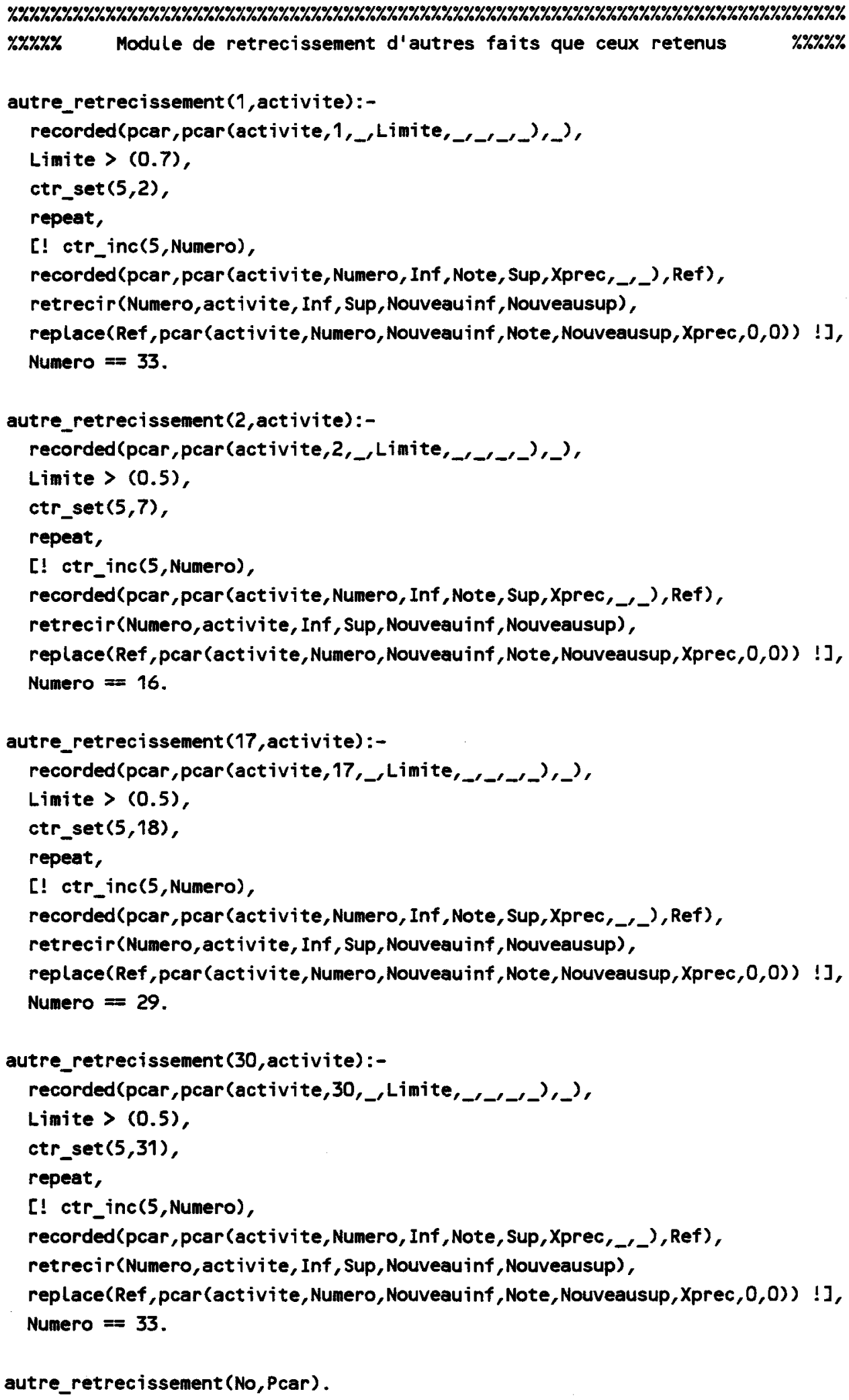


\%\%\%\%\%\%\%\%\%\%\%\%\%\%\%\%\%\%\%\%\%\%\%\%\%\%\%\%\%\%\%\%\%\%\%\%\%\%\%\%\%\%\%\%\%\%\%\%\%\%\%\%\%\%\%\%\%\%\%\%\%\%\%\%\%\%

$\% \% \% \%$ Module de retrecissement et d'elargissement des intervalles $\% \% \%$

retrecir(No, programme, Inf, Sup, Nouveauinf, Nouveausup):-

Nouveauinf is Int +0.1 ,

Nouveausup is Sup -0.1 .

retrecir(1, act ivite, Inf, Sup, Nouveauinf, Nouveausup):-

Nouveauinf is Inf +0.2 ,

Nouveausup is Sup.

retrecir (No, activite, Inf, Sup, Nouveauinf, Nouveausup) :-

Nouveauinf is Inf +0.5 ,

Nouveausup is Sup -0.5 .

retrecir (5, resultat, Inf, Sup, Nouveauinf, Nouveausup):-

Nouveauinf is Inf,

Nouveausup is Sup.

retrecir(6, resul tat, Inf, Sup, Nouveauinf, Nouveausup):Nouveauinf is Inf,

Nouveausup is Sup.

retrecir (No, resultat, Inf, Sup, Nouveauinf, Nouveausup):-

Nouveauinf is Inf +0.05 ,

Nouveausup is Sup -0.05 .

retrecir(No, contexte, Inf, Sup, Nouveauinf, Nouveausup):-

Nouveauinf is Inf,

Nouveausup is Sup.

elargir(programme):-

ctr_set $(0,1)$,

repeat,

[! ctr_inc(0, Controleur),

recorded (pcar, pcar (programme, Controleur,_,Pi,_,Pprec, Somme, Nb), Ref), recorded ( Limite, limite (programme, Controleur, Inf, Sup),_),

replace (Ref, pcar (programme, Controleur, Inf, Pi, Sup, Pprec, Somme, Nb)) !],

Controleur $==15$.

elargir(contexte):-

ctr_set $(0,1)$,

repeat,

[! ctr_inc(0, Controleur),

recorded (pcar, pcar (contexte, Controleur, , $\mathrm{Ci}$, , Cprec, Somme, Nb), Ref),

recorded(Limite, Limite(contexte, Controleur, Inf, Sup),_),

replace (Ref, pcar (contexte, Controleur, Inf, $C i$, Sup, Cprec, Somme, Nb)) !],

Controleur $==9$. 


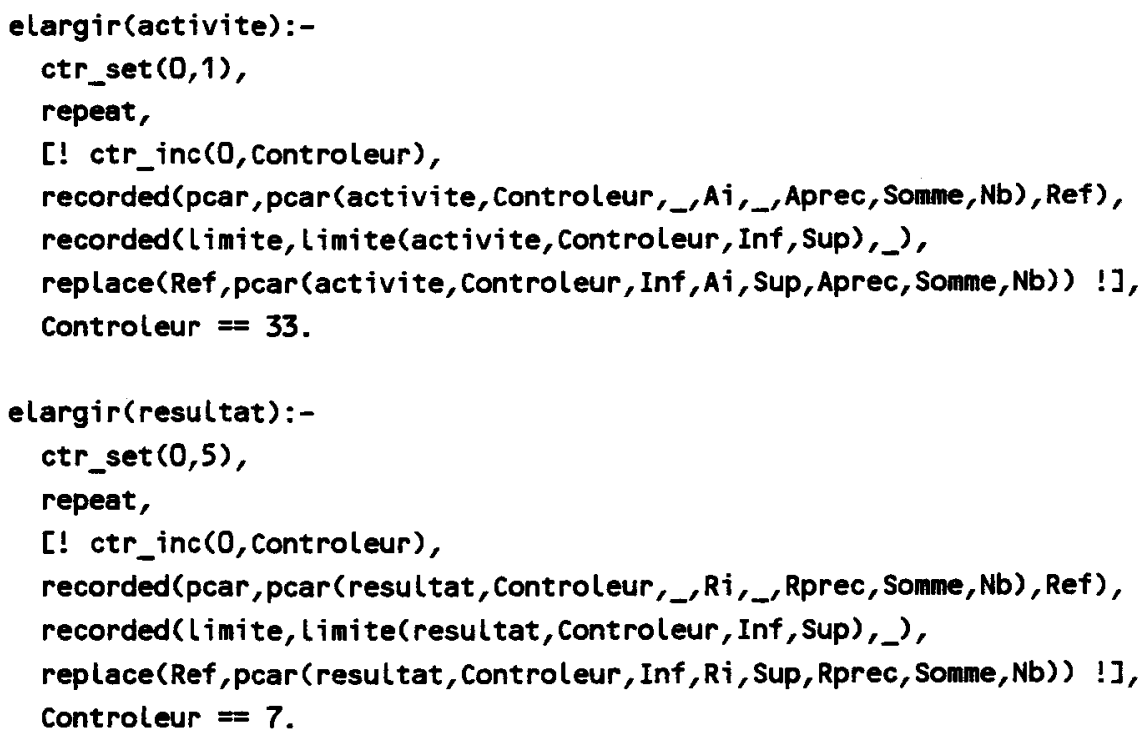

changer_norme(resultat).

changer_norme (programme).

changer_norne(contexte).

changer_norme(activite):-

demander_note.

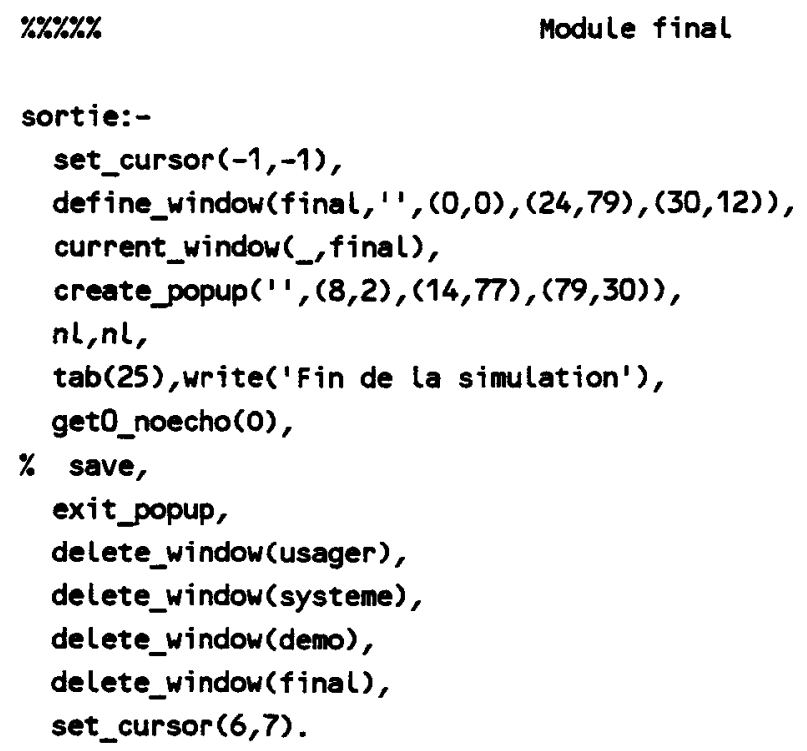




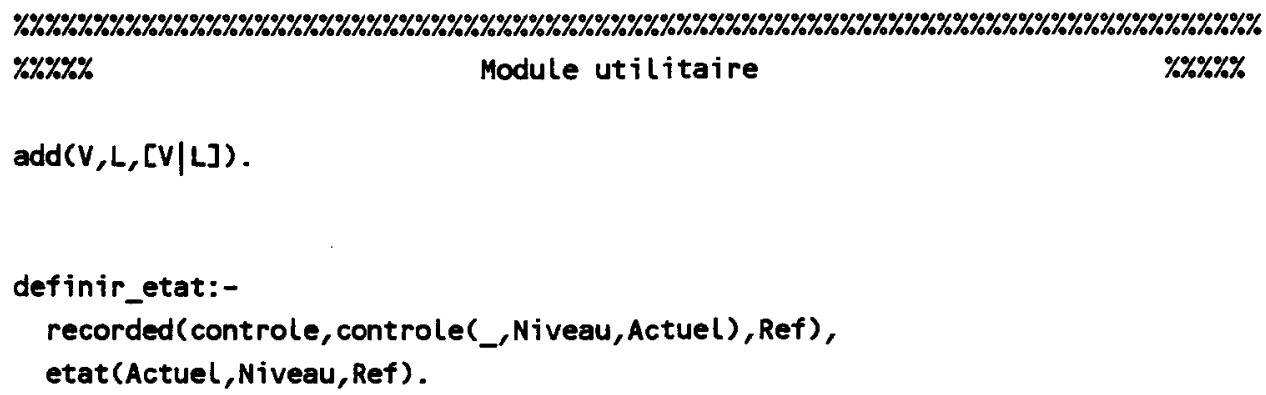

$\operatorname{add}(V, L,[V \mid L])$.

definir_etat:-

recorded(controle, controle(_,Niveau,Actuel), Ref), etat (Actuel, Niveau, Ref).

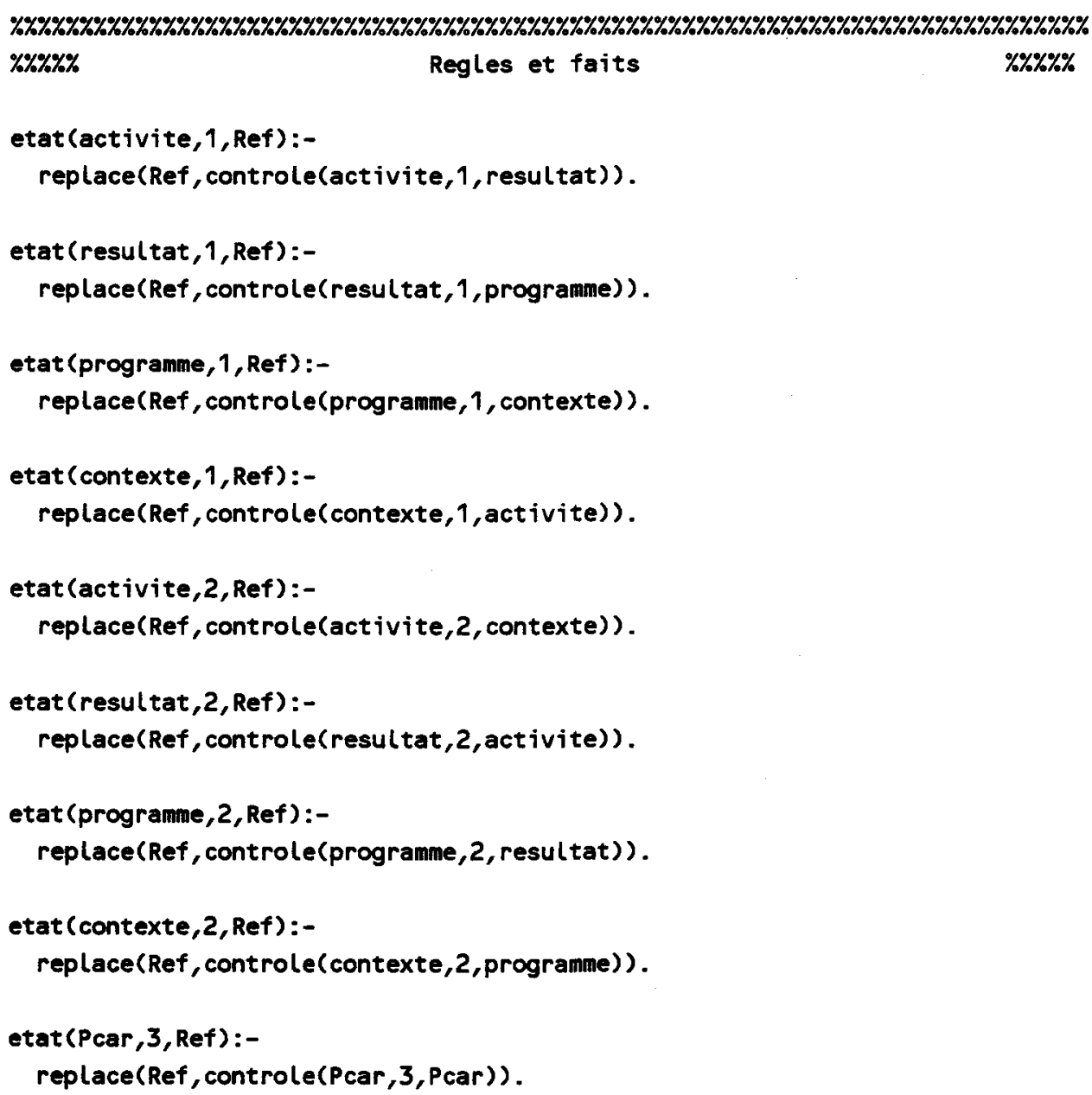




\section{PARTIE 2}

\section{LES FICHIERS DE PROLIN}




\section{PARTIE 2}

\section{LES FICHIERS DE PROLIN}

\section{1: Le fichier "PCAR.ARI"}

pcar(programme, $1,0.4,0,1,0,0,0$ ). pcar(programme, $2,0.4,0,0.9,0,0,0)$. pcar(programme, $3,0.1,0,0.8,0,0,0$ ). pcar(programme, $4,0.5,0,0.8,0,0,0)$. pcar(programme, $5,0.6,0,0.8,0,0,0$ ). pcar(programme, $6,0.6,0,0.8,0,0,0)$. pcar(programme, $7,0.6,0,0.8,0,0,0$ ). pcar(programme, $8,0.1,0,0.7,0,0,0)$. pcar(programme, $9,0.1,0,0.7,0,0,0)$. pcar(programme, 10,0.1,0,0.7,0,0,0). pcar(programme, 11,0.1,0,0.7,0,0,0). pcar(programme, 12,0.1,0,0.7,0,0,0). pcar(programme, 13,0.1,0,0.7,0,0,0). pcar(programme, 14,0.1,0,0.7,0,0,0). pcar(programme, 15,0.1,0,0.7,0,0,0). pcar(contexte, 1,0.1,0,0.8,0,0,0). pcar (contexte, 2, 0.2,0,0.85,0,0,0). pcar(contexte, $3,0.3,0,1.0,0,0,0)$. pcar(contexte,4,0.1,0,0.8,0,0,0). pcar (contexte, $5,0.2,0,0.85,0,0,0$ ). pcar(contexte, 6,0.3,0,1.0,0,0,0). pcar(contexte, $7,0.1,0,0.8,0,0,0$ ). pcar (contexte, $8,0.2,0,0.85,0,0,0$ ). pcar(contexte,9,0.3,0,1.0,0,0,0). pcar (activite, 1,0.1,0,1,0,0,0). pcar(activite, $2,0.4,0,1,0,0,0$ ). pcar(activite, 3,0.5,0,0.9,0,0,0). 
pcar(activite, 4, 0.5,0,0.9,0,0,0). pcar(activite, 5, 0.5,0,0.9,0,0,0). pcar(activite, $6,0.5,0,0.9,0,0,0)$. pcar(activite, $7,0.55,0,0.85,0,0,0$ ). pcar(activite, $8,0.55,0,0.85,0,0,0$ ). pcar(activite, $9,0.55,0,0.85,0,0,0$ ). pcar(activite, 10,0.55,0,0.85,0,0,0). pcar(activite, 11, 0.55,0,0.85,0,0,0). pcar(activite, 12,0.55,0,0.85,0,0,0). pcar(activite, 13,0.6,0,0.8,0,0,0). pcar(activite, 14,0.6,0,0.8,0,0,0). pcar(activite, 15,0.6,0,0.8,0,0,0). pcar(activite, 16,0.6,0,0.8,0,0,0). pcar(activite, 17,0.6,0,0.8,0,0,0). pcar(activite, 18,0.6,0,0.8,0,0,0). pcar(activite, 19,0.6,0,0.8,0,0,0). pcar(activite,20,0.6,0,0.8,0,0,0). pcar(activite, 21, 0.6,0,0.8,0,0,0). pcar(activite,22, $0.4,0,0.7,0,0,0$ ). pcar(activite, 23,0.4,0,0.7,0,0,0). pcar(activite, 24, $0.4,0,0.7,0,0,0$ ). pcar(activite,25,0.4,0,0.7,0,0,0). pcar(activite, 26, 0.4,0,0.7,0,0,0). pcar(activite,27,0.4,0,0.7,0,0,0). pcar(activite,28,0.4,0,0.7,0,0,0). pcar(activite, $29,0.4,0,0.7,0,0,0$ ). pcar(activite,30,0.2,0,0.8,0,0,0). pcar(activite, $31,0.4,0,0.6,0,0,0)$. pcar(activite,32,0.4,0,0.6,0,0,0). pcar(activite,33,0.4,0,0.6,0,0,0). pcar(resultat, $1,0.3,0,1,0,0,0)$. pcar(resultat,2,0.3,0,1,0,0,0). pcar(resultat, $3,0.3,0,1,0,0,0)$. pcar(resultat,4,0.3,0,1,0,0,0). pcar(resultat,5,0.3,0,1.0,0,0,0). pcar(resultat,6,0.3,0,1.0,0,0,0). pcar(resultat,7,0.3,0,0.8,0,0,0). pcar(resultat,8,0.3,0,0.9,0,0,0). limite(programme, 1,0.4,1). limite(programme,2,0.4,0.9). limite(programme,3,0.1,0.8). 
limite(programme, 4,0.5,0.8). limite(programme, $5,0.6,0.8$ ). limite(programme,6,0.6,0.8). limite(programme,7,0.6,0.8). limite(programme,8,0.1,0.7). limite(programme,9,0.1,0.7). limite(programme, 10,0.1,0.7). limite(programme, 11,0.1,0.7). limite(programme, 12,0.1,0.7). limite(programme, 13,0.1,0.7). limite(programme, 14,0.1,0.7). limite(programme, 15,0.1,0.7). limite(contexte, $1,0.1,0.8$ ). limite(contexte,2,0.2,0.85). limite (contexte, $3,0.3,1.0$ ). limite(contexte, $4,0.1,0.8$ ). limite(contexte,5,0.2,0.85). limite(contexte, 6,0.3,1.0). limite(contexte, $7,0.1,0.8$ ). limite (contexte, $8,0.2,0.85$ ). limite(contexte, $9,0.3,1.0$ ). limite(activite, 1,0.1,1). limite(activite, 2,0.4,1). limite(activite, $3,0.5,0.9$ ). limite(activite, $4,0.5,0.9)$. limite(activite, $5,0.5,0.9$ ). limite (activite, 6,0.5,0.9). limite (activite, $7,0.55,0.85$ ). limite(activite, $8,0.55,0.85$ ). limite(activite, $9,0.55,0.85$ ). limite(activite, 10,0.55,0.85). limite(activite, 11,0.55,0.85). limite(activite, 12,0.55,0.85). limite(activite, 13,0.6,0.8). limite(activite, 14,0.6,0.8). limite(activite, 15,0.6,0.8). limite(activite, 16,0.6,0.8). limite(activite, 17,0.6,0.8). limite(activite, 18,0.6,0.8). limite(activite, 19,0.6,0.8). limite(activite,20,0.6,0.8). 
limite(activite, $21,0.6,0.8$ ).

limite(activite, 22, 0.4,0.7).

limite(activite,23,0.4,0.7).

limite(activite, 24,0.4,0.7).

limite(activite, 25,0.4,0.7).

limite(activite,26,0.4,0.7).

limite(activite,27,0.4,0.7).

limite(activite,28,0.4,0.7).

limite(activite, 29,0.4,0.7).

limite(activite, $30,0.2,0.8$ ).

limite(activite,31,0.4,0.6).

limite(activite,32,0.4,0.6).

limite(activite,33,0.4,0.6).

limite(resultat, 1,0.3,1).

limite(resultat,2,0.3,1).

limite(resultat,3,0.3,1).

limite(resultat, 4,0.3,1).

limite(resultat,5,0.3,1.0).

limite(resultat,6,0.3,1.0).

limite(resultat,7,0.3,1).

limite(resultat,8,0.3,0.9).

\%pcar(fin, $0,0,0,0,0,0,0$ ).

etudiant(bidon, bidon).

psy(bidon,bidon,bidon).

controle(contexte, 1 , contexte).

plus_compatible(programme,2,0).

plus_compatible(contexte, 1,0 ).

plus_compatible(activite, 1,0 ).

plus_compatible(resultat, 1,0 ). 


\section{2: Le fichier "NORMES.ARI"}

$$
\begin{aligned}
& \text { norme(a1p1,1). } \\
& \text { norme }(a 2 p 3,1) \text {. } \\
& \text { norme (a2p4,0.8). } \\
& \text { norme }(a 3 p 3,0.8) \text {. } \\
& \text { norme (a3p6,0.4). } \\
& \text { norme }(a 4 p 3,0.8) \text {. } \\
& \text { norme }(a 4 p 7,0.4) \text {. } \\
& \text { norme (a5p3,0.8). } \\
& \text { norme }(a 5 p 7,0.4) \text {. } \\
& \text { norme }(a 6 p 3,0.8) \text {. } \\
& \text { norme }(a 6 p 7,0.4) \text {. } \\
& \text { norme }(a 7 p 3,0.6) \text {. } \\
& \text { norme }(a 7 p 5,1) \text {. } \\
& \text { norme }(a 8 p 3,0.6) \text {. } \\
& \text { norme (a8p6,0.8). } \\
& \text { norme (a9p3,0.6). } \\
& \text { norme }(a 9 p 7,0.6) \text {. } \\
& \text { norme (a10p3,0.6). } \\
& \text { norme (a10p6,0.8). } \\
& \text { norme }(a 11 \mathrm{p3}, 0.6) \text {. } \\
& \text { norme }(a 11 p 7,0.6) \text {. } \\
& \text { norme }(a 12 p 3,0.6) \text {. } \\
& \text { norme }(a 12 p 7,0.6) \text {. } \\
& \text { norme (a13p3,0.4). } \\
& \text { norme (a13p5,0.8). } \\
& \text { norme }(a 14 p 3,0.4) \text {. } \\
& \text { norme (a14p5,0.8). } \\
& \text { norme }(a 15 p 3,0.4) \text {. } \\
& \text { norme }(a 15 p 6,1) \text {. } \\
& \text { norme }(a 16 p 3,0.4) \text {. } \\
& \text { norme }(a 16 p 7,1) \text {. } \\
& \text { norme(a17p2,0.6). } \\
& \text { norme }(a 17 p 3,1) \text {. } \\
& \text { norme }(a 17 p 4,0.9) \text {. } \\
& \text { norme(a17p5,0.4). }
\end{aligned}
$$


norme $(a 17 p 6,0.6)$.

norme $(a 17 p 7,0.6)$.

norme $(a 17 p 8,0.4)$.

norme (a17p9,0.4).

norme (a17p10,0.4).

norme (a17p11,0.4).

norme(a17p12,0.4).

norme (a17p13,0.4).

norme (a17p14,0.4).

norme (a17p15,0.4).

norme (a18p3,0.8).

norme (a18p5,0.6).

norme (a18p8,0.8).

norme (a18p9,0.8).

norme (a19p3,0.8).

norme (a19p5,0.6).

norme(a19p10,0.8).

norme (a19p11,0.8).

norme (a20p3,0.8).

norme (a20p6,0.8).

norme(a20p12,0.8).

norme(a20p13,0.8).

norme (a21p3,0.8).

norme $(221 \mathrm{p} 7,0.8)$.

norme(a21p14,0.8).

norme (a21p15,0.8).

norme (a22p3,0.5).

norme $(222 p 5,0.6)$.

norme $(a 22 p 8,1)$.

norme $(223 p 3,0.5)$.

norme (a23p5,0.6).

norme (a23p9,1).

norme $(224 p 3,0.5)$.

norme $(224 p 5,0.6)$.

norme $(224 p 10,1)$.

norme $(225 p 3,0.5)$.

norme (225p5,0.6).

norme (a25p11,1).

norme $(226 p 3,0.5)$.

norme $(226 p 6,0.6)$.

norme(a26p12,1). 


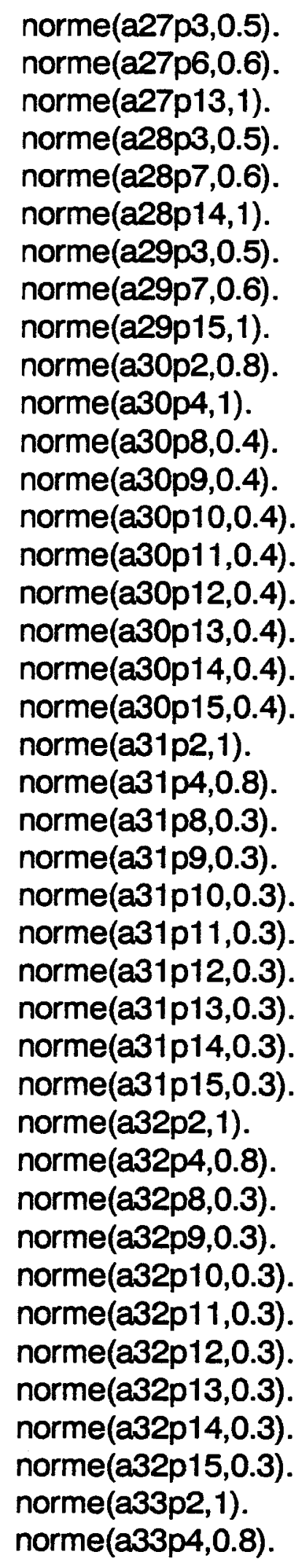


norme $(233 p 8,0.3)$.

norme (a33p9,0.3).

norme (a33p10,0.3).

norme (a33p11,0.3).

norme (a33p12,0.3).

norme (a33p13,0.3).

norme (a33p14,0.3).

norme (a33p15,0.3).

norme (p1a2,0.8).

norme (p1a3,1).

norme (p1a4,1).

norme (p1a5,1).

norme (p1a6,1).

norme (p2a1,0.4).

norme (p2a2,0.6).

norme (p2a30,1).

norme (p2a31,1).

norme (p2a32,1).

norme (p2a33,1).

norme(p3a1,0.8).

norme (p3a2,1).

norme (p3a3,1).

norme (p3a4,1).

norme (p3a5,1).

norme (p3a6,1).

norme (p3a7,0.8).

norme (p3a8,0.8).

norme (p3a9,0.8).

norme(p3a10,0.8).

norme(p3a11,0.8).

norme(p3a12,0.8).

norme(p3a13,0.4).

norme(p3a14,0.4).

norme(p3a15,0.4).

norme (p3a16,0.4).

norme (p3a17,1).

norme(p3a18,0.6).

norme (p3a19,0.6).

norme (p3a20,0.6).

norme (p3a21,0.6).

norme(p3a22,0.2). 
norme (p3a23,0.2). norme(p3a24,0.2). norme (p3a25,0.2). norme(p3a26,0.2). norme (p3a27,0.2). norme $(p 3 a 28,0.2)$. norme (p3a29,0.2). norme $(p 3 a 30,0.9)$. norme (p3a31,0.8). norme (p3a32,0.8). norme (p3a33,0.8). norme (p4a1,0.2). norme (p4a2,0.4). norme $(p 4 a 30,0.9)$. norme (p4a31,1). norme (p4a32,1). norme (p4a33,1). norme (p5a1,0.5). norme (p5a2,0.6). norme (p5a3,0.3). norme (p5a4,0.2). norme (p5a5,0.1). norme (p5a6,0.1). norme (p5a7,1). norme (p5a8,0.7). norme (p5a9,0.7). norme(p5a10,0.7). norme(p5a11,0.7). norme (p5a12,0.5). norme(p5a13,0.8). norme (p5a14,0.8). norme (p5a15,0.6). norme(p5a16,0.4). norme (p5a17,0.4). norme(p5a18,0.8). norme (p5a19,0.8). norme (p5a22,0.8). norme (p5a23,0.8). norme (p5a24,0.8). norme (p5a25,0.8). norme (p5a26,0.6). 
norme (p5a27,0.6).

norme (p5a30,0.8).

norme (p6a1,0.5).

norme (p6a2,0.6).

norme (p6a3,0.4).

norme (p6a4,0.3).

norme (p6a5,0.3).

norme (p6a6,0.3).

norme (p6a7,0.9).

norme (p6a8,0.9).

norme(p6a9,0.6).

norme (p6a10,0.9).

norme(p6a11,0.6).

norme(p6a12,0.7).

norme (p6a15,0.8).

norme(p6a16,0.6).

norme(p6a17,0.5).

norme (p6a18,0.4).

norme(p6a19,0.4).

norme (p6a20,0.8).

norme (p6a21,0.4).

norme (p6a22,0.6).

norme (p6a23,0.6).

norme (p6a24,0.6).

norme (p6a25,0.6).

norme (p6a26,0.8).

norme (p6a27,0.8).

norme (p6a28,0.6).

norme (p6a29,0.6).

norme (p7a1,0.5).

norme (p7a2,0.6).

norme (p7a4,0.6).

norme (p7a5,0.6).

norme $(p 7 a 6,0.6)$.

norme (p7a7,0.5).

norme (p7a8,0.5).

norme (p7a9,0.9).

norme(p7a10,0.5).

norme (p7a11,0.9).

norme (p7a12,0.9).

norme $(p 7 a 15,0.6)$. 
norme (p7a16,0.8). norme(p7a17,0.6). norme (p7a20,0.4). norme (p7a21,0.8). norme (p7a26,0.6). norme (p7a27,0.6). norme (p7a28,0.8). norme (p7a29,0.8). norme (p8a1,0.2). norme(p8a2,0.3). norme (p8a3,0.5). norme $(p 8 a 4,0.5)$. norme $(p 8 a 5,0.5)$. norme (p8a7,0.8). norme $(p 8 a 8,0.8)$. norme (p8a9,0.7). norme (p8a13,0.9). norme (p8a17,0.2). norme (p8a18,1). norme (p8a22,1). norme (p8a30,0.8). norme(p9a1,0.2). norme (p9a2,0.3). norme (p9a3,0.5). norme $(p 9 a 4,0.5)$. norme $(p 9 a 5,0.5)$. norme (p9a7,0.8). norme(p9a8,0.8). norme (p9a9,0.7). norme (p9a13,0.9). norme (p9a17,0.2). norme (p9a18,1). norme (p9a23,1). norme (p9a30,0.8). norme (p10a1,0.2). norme (p10a2,0.3). norme (p10a3,0.5). norme (p10a4,0.5). norme (p10a6,0.5). norme (p10a7,0.8). norme(p10a10,0.8). 
norme(p10a11,0.7).

norme(p10a14,0.9).

norme (p10a17,0.2).

norme(p10a19,1).

norme (p10a24,1).

norme(p10a30,0.8).

norme (p11a1,0.2).

norme(p11a2,0.3).

norme (p11a3,0.5).

norme (p11a4,0.5).

norme (p11a6,0.5).

norme (p11a7,0.5).

norme(p11a10,0.8).

norme(p11a11,0.7).

norme(p11a14,0.9).

norme(p11a17,0.2).

norme(p11a19,1).

norme (p11a25,1).

norme (p11a30,0.8).

norme(p12a1,0.2).

norme (p12a2,0.3).

norme(p12a3,0.5).

norme(p12a5,0.5).

norme (p12a6,0.5).

norme(p12a8,0.9).

norme (p12a10,0.9).

norme(p12a12,0.7).

norme(p12a15,0.9).

norme(p12a17,0.2).

norme (p12a20,1).

norme $(p 12 a 26,1)$.

norme(p13a1,0.2).

norme (p13a2,0.3).

norme(p13a3,0.5).

norme(p13a5,0.5).

norme (p13a6,0.5).

norme (p13a8,0.9).

norme (p13a10,0.9).

norme(p13a12,0.7).

norme (p13a15,0.9).

norme(p13a17,0.2). 
norme $(p 13 a 20,1)$. norme (p13a27,1). norme (p14a1,0.2). norme (p14a2,0.3). norme (p14a4,0.5). norme (p14a5,0.5). norme (p14a6,0.5). norme (p14a9,0.9). norme (p14a11,0.9). norme(p14a12,0.9). norme (p14a16,0.9). norme $(p 14 a 17,0.2)$. norme (p14a21,1). norme (p14a28,1). norme(p15a1,0.2). norme(p15a2,0.3). norme (p15a4,0.5). norme (p15a5,0.5). norme(p15a6,0.5). norme (p15a9,0.9). norme (p15a11,0.9). norme(p15a12,0.9). norme (p15a16,0.9). norme (p15a17,0.2). norme (p15a21,1). norme (p15a29,1). norme $(a 1 c 2,0.5)$. norme $(\mathrm{a} 1 \mathrm{c} 3,1)$. norme $(a 1 c 5,0.5)$. norme $(\mathrm{a} 1 \mathrm{c} 6,1)$. norme $(a 1 c 8,0.5)$. norme $(a 1 c 9,1)$. norme $(a 2 c 1,0.1)$. norme $(22 \mathrm{c} 2,0.7)$. norme $(a 2 c 3,1)$. norme $(22 c 4,0.1)$. norme (a2c5,0.7). norme $(a 2 c 6,1)$. norme $(a 2 c 7,0.1)$. norme $(22 \mathrm{c} 8,0.7)$. norme $(a 2 c 9,1)$. 
norme $(a 3 c 1,0.2)$.

norme $(a 3 c 2,0.7)$.

norme $(23 c 3,1)$.

norme $(a 3 c 4,0.2)$.

norme $(23 c 5,0,7)$.

norme $(23 c 6,1)$.

norme $(a 3 c 7,0.2)$.

norme $(a 3 c 8,0.7)$.

norme $(a 3 c 9,1)$.

norme $(a 4 c 1,0.2)$.

norme $(a 4 c 2,0.7)$.

norme $(24 c 3,1)$.

norme $(a 4 c 4,0.2)$.

norme $(a 4 c 5,0.7)$.

norme $(a 4 c 6,1)$.

norme $(a 4 c 7,0.2)$.

norme $(a 4 c 8,0.7)$.

norme $(a 4 c 9,1)$.

norme $(a 5 c 1,0.2)$.

norme $(25 \mathrm{c} 2,0.7)$.

norme $(25 \mathrm{c} 3,1)$.

norme $(a 5 c 4,0.2)$.

norme (a5c5,0.7).

norme $(a 5 c 6,1)$.

norme $(a 5 c 7,0.2)$.

norme $(25 c 8,0.7)$.

norme $(25 \mathrm{c} 9,1)$.

norme (a6c1,0.2).

norme $(a 6 c 2,0.7)$.

norme $(a 6 c 3,1)$.

norme $(a 6 c 4,0.2)$.

norme $(a 6 c 5,0.7)$.

norme $(a 6 c 6,1)$.

norme $(a 6 c 7,0.2)$.

norme $(a 6 c 8,0.7)$.

norme $(a 6 c 9,1)$.

norme $(a 7 c 1,0.3)$.

norme $(a 7 c 2,0.7)$.

norme $(27 c 3,1)$.

norme $(a 7 c 4,0.3)$.

norme $(a 7 c 5,0.7)$. 
norme(a7c6,1).

norme $(a 7 c 7,0.3)$.

norme $(a 7 c 8,0.7)$.

norme $(a 7 c 9,1)$.

norme (a8c1,0.3).

norme $(a 8 c 2,0.7)$.

norme $(a 8 c 3,1)$.

norme $(a 8 c 4,0.3)$.

norme $(a 8 c 5,0.7)$.

norme $(a 8 c 6,1)$.

norme $(a 8 c 7,0.3)$.

norme $(28 c 8,0.7)$.

norme $(a 8 c 9,1)$.

norme $(a 9 c 1,0.3)$.

norme $(a 9 c 2,0.7)$.

norme (a9c3,1).

norme $(a 9 c 4,0.3)$.

norme $(a 9 c 5,0.7)$.

norme $(a 9 c 6,1)$.

norme (a9c7,0.3).

norme $(a 9 c 8,0.7)$.

norme $(a 9 c 9,1)$.

norme $(\mathrm{a} 10 \mathrm{c} 1,0.3)$.

norme(a10c2,0.7).

norme $(a 10 c 3,1)$.

norme $($ a10c4,0.3).

norme $(\mathrm{a} 10 \mathrm{c5}, 0.7)$.

norme $(a 10 c 6,1)$.

norme $(a 10 \mathrm{c} 7,0.3)$.

norme $(a 10 \mathrm{c} 8,0.7)$.

norme (a10c9,1).

norme $(a 11 \mathrm{c} 1,0.3)$.

norme(a11c2,0.7).

norme $(a 11 \mathrm{c} 3,1)$.

norme $(a 11 \mathrm{c} 4,0.3)$.

norme(a11c5,0.7).

norme $(a 11 \mathrm{c} 6,1)$.

norme $(a 11 c 7,0.3)$.

norme $($ a11c8,0.7).

norme $(a 11 \mathrm{c} 9,1)$.

norme $(a 12 c 1,0.3)$. 
norme $(a 12 c 2,0.7)$.

norme $(a 12 c 3,1)$.

norme (a12c4,0.3).

norme (a12c5,0.7).

norme $(\mathrm{a} 12 \mathrm{c} 6,1)$.

norme $(a 12 c 7,0.3)$.

norme (a12c8,0.7).

norme $(\mathrm{a} 12 \mathrm{c9}, 1)$.

norme $(a 13 c 1,0.5)$.

norme (a13c2,0.7).

norme $(a 13 c 3,0.9)$.

norme $(a 13 c 4,0.5)$.

norme (a13c5,0.7).

norme $(a 13 c 6,0.9)$.

norme $(a 13 c 7,0.5)$.

norme (a13c8,0.7).

norme (a13c9,0.9).

norme $(\mathrm{a} 14 \mathrm{c1}, 0.5)$.

norme $(a 14 c 2,0.7)$.

norme $(a 14 c 3,0.9)$.

norme $(a 14 c 4,0.5)$.

norme (a14c5,0.7).

norme $(a 14 c 6,0.9)$.

norme $(a 14 c 7,0.5)$.

norme (a14c8,0.7).

norme $(a 14 c 9,0.9)$.

norme $(a 15 c 1,0.5)$.

norme $(a 15 c 2,0.7)$.

norme $(a 15 c 3,0.9)$.

norme $(a 15 c 4,0.5)$.

norme $(215 c 5,0.7)$.

norme $(a 15 c 6,0.9)$.

norme $(a 15 c 7,0.5)$.

norme $(a 15 c 8,0.7)$.

norme $(215 c 9,0.9)$.

norme (a16c1,0.5).

norme (a16c2,0.7).

norme $(a 16 c 3,0.9)$.

norme (a16c4,0.5).

norme (a16c5,0.7).

norme $(a 16 c 6,0.9)$. 
norme $(a 16 c 7,0.5)$. norme (a16c8,0.7). norme $(a 16 c 9,0.9)$. norme (a17c1,0.6). norme(a17c2,0.8). norme $(a 17 c 3,1)$. norme (a17c4,0.6). norme (a17c5,0.8). norme (a17c6,1).

norme $(a 17 c 7,0.6)$. norme (a17c8,0.8). norme $(a 17 c 9,1)$.

norme (a18c1,0.7). norme (a18c2,0.8). norme (a18c3,0.9). norme (a18c4,0.7). norme(a18c5,0.8). norme (a18c6,0.9). norme $(a 18 c 7,0.7)$. norme $(a 18 c 8,0.8)$. norme $(a 18 c 9,0.9)$. norme $(a 19 c 1,0.7)$. norme (a19c2,0.8). norme (a19c3,0.9). norme (a19c4,0.7). norme (a19c5,0.8). norme (a19c6,0.9). norme (a19c7,0.7). norme (a19c8,0.8). norme $(a 19 c 9,0.9)$. norme $(220 c 1,0.7)$. norme $(220 \mathrm{c} 2,0.8)$. norme $(220 \mathrm{c} 3,0.9)$. norme (a20c4,0.7). norme (a20c5,0.8). norme $(220 \mathrm{c} 6,0.9)$. norme $(220 \mathrm{c} 7,0.7)$. norme $(220 \mathrm{c} 8,0.8)$. norme $(220 \mathrm{c} 9,0.9)$. norme (a21c1,0.7). norme $(221 \mathrm{c} 2,0.8)$. 
norme $(221 c 3,0.9)$. norme $(221 c 4,0.7)$. norme $(221 c 5,0.8)$. norme $(a 21 c 6,0.9)$. norme $(221 \mathrm{c} 7,0.7)$. norme $(221 \mathrm{c} 8,0.8)$. norme $(221 c 9,0.9)$. norme $(a 22 c 1,0.8)$. norme $(a 22 c 2,0.6)$. norme $(a 22 c 3,0.4)$. norme $(222 c 4,0.8)$. norme $(222 c 5,0.6)$. norme $(a 22 c 6,0.4)$. norme $(222 c 7,0.8)$. norme $(a 22 c 8,0.6)$. norme $(222 c 9,0.4)$. norme $(223 c 1,0.8)$. norme (a23c2,0.6). norme $(223 c 3,0.4)$. norme $(a 23 c 4,0.8)$. norme (a23c5,0.6). norme $(a 23 c 6,0.4)$. norme $(a 23 c 7,0.8)$. norme $(223 c 8,0.6)$. norme $(223 c 9,0.4)$. norme $(a 24 c 1,0.8)$. norme $(a 24 c 2,0.6)$. norme $(a 24 c 3,0.4)$. norme $(a 24 c 4,0.8)$. norme $(224 c 5,0.6)$. norme $(224 c 6,0.4)$. norme $(224 c 7,0.8)$. norme $(224 c 8,0.6)$. norme $(a 24 c 9,0.4)$. norme (a25c1,0.8). norme $(225 c 2,0.6)$. norme $(225 c 3,0.4)$. norme (a25c4,0.8). norme $(a 25 c 5,0.6)$. norme $(225 c 6,0.4)$. norme $(225 c 7,0.8)$. 
norme $(225 \mathrm{c} 8,0.6)$. norme $(225 c 9,0.4)$. norme $(226 c 1,0.8)$. norme $(226 c 2,0.6)$. norme $(226 c 3,0.4)$. norme (a26c4,0.8). norme (a26c5,0.6). norme $(226 c 6,0.4)$. norme $(226 c 7,0.8)$. norme $(226 c 8,0.6)$. norme $(226 c 9,0.4)$. norme (a27c1,0.8). norme $(227 c 2,0.6)$. norme $(227 c 3,0.4)$. norme $(227 c 4,0.8)$. norme $(a 27 c 5,0.6)$. norme $(227 c 6,0.4)$. norme $(227 c 7,0.8)$. norme $(a 27 c 8,0.6)$. norme $(227 c 9,0.4)$. norme $(a 28 c 1,0.8)$. norme $(228 \mathrm{c} 2,0.6)$. norme $(228 c 3,0.4)$. norme $(228 c 4,0.8)$. norme $(228 c 5,0.6)$. norme $(228 c 6,0.4)$. norme $(a 28 c 7,0.8)$. norme $(228 c 8,0.6)$. norme $(a 28 c 9,0.4)$. norme $(a 29 c 1,0.8)$. norme $(229 \mathrm{c} 2,0.6)$. norme $(229 c 3,0.4)$. norme (a29c4,0.8). norme (a29c5,0.6). norme $(229 \mathrm{c} 6,0.4)$. norme $(229 c 7,0.8)$. norme $(a 29 c 8,0.6)$. norme $(a 29 c 9,0.4)$. norme $(230 \mathrm{c} 1,0.6)$. norme $(230 \mathrm{c} 2,0.8)$. norme $(230 \mathrm{c} 3,1)$. 
norme (a30c4,0.6). norme (a30c5,0.8). norme $(230 \mathrm{c} 6,1)$. norme $(a 30 c 7,0.6)$. norme $(a 30 \mathrm{c} 8,0.8)$. norme $(a 30 c 9,1)$. norme $(a 31 \mathrm{c} 1,1)$. norme (a31c2,0.8). norme $(a 31 c 3,0.6)$. norme $(a 31 \mathrm{c} 4,0.2)$. norme $(231 \mathrm{c5}, 0.4)$. norme $(231 \mathrm{c} 6,0.6)$. norme $(231 \mathrm{c} 7,0.2)$. norme $(231 \mathrm{c} 8,0.4)$. norme (a31c9,0.6). norme $(232 c 1,0.2)$. norme $(a 32 c 2,0.4)$. norme $(a 32 c 3,0.6)$. norme $(232 c 4,1)$.

norme $(232 c 5,0.8)$. norme $(a 32 c 6,0.6)$. norme (a32c7,0.2). norme $(a 32 c 8,0.4)$. norme $(232 \mathrm{c} 9,0.6)$. norme $(a 33 c 1,0.2)$. norme $(233 \mathrm{c} 2,0.4)$. norme $(233 c 3,0.6)$. norme (a33c4,0.2). norme $(233 c 5,0.4)$. norme $(233 \mathrm{c} 6,0.6)$. norme $(233 c 7,1)$. norme $(a 33 c 8,0.8)$. norme $(233 c 9,0.6)$. norme (c1p2,1). norme (c1p3,0.3). norme (c1p4,0.9). norme(c1p5,0.4). norme (c1p6,0.3). norme (c1p7,0.2). norme (c1p8,0.6). norme (c1p9,0.6). 
norme(c1p10,0.6). norme(c1p11,0.6). norme(c1p12,0.4). norme(c1p13,0.4). norme(c1p14,0.2). norme(c1p15,0.2). norme (c2p1,0.2). norme (c2p2,0.3). norme (c2p3,0.6). norme(c2p4,0.6). norme (c2p5,0.9). norme(c2p6,0.8). norme (c2p7,0.7). norme (c2p8,0.9). norme (c2p9,0.9). norme (c2p10,0.9). norme(c2p11,0.9). norme(c2p12,0.8). norme(c2p13,0.8). norme(c2p14,0.7). norme(c2p15,0.7). norme (c3p1,1). norme $(c 3 p 3,1)$. norme (c3p4,0.3). norme (c3p5,1). norme (c3p6,1). norme $(3 \mathrm{p} 7,1)$. norme (c3p8,0.7). norme (c3p9,0.7). norme(c3p10,0.7). norme(c3p11,0.7). norme(c3p12,0.8). norme(c3p13,0.8). norme (c3p14,0.9). norme(c3p15,0.9). norme (c4p2,1). norme (c4p3,0.3). norme $(c 4 p 4,0.9)$. norme (c4p5,0.4). norme (c4p6,0.3). norme $(c 4 p 7,0.2)$. 


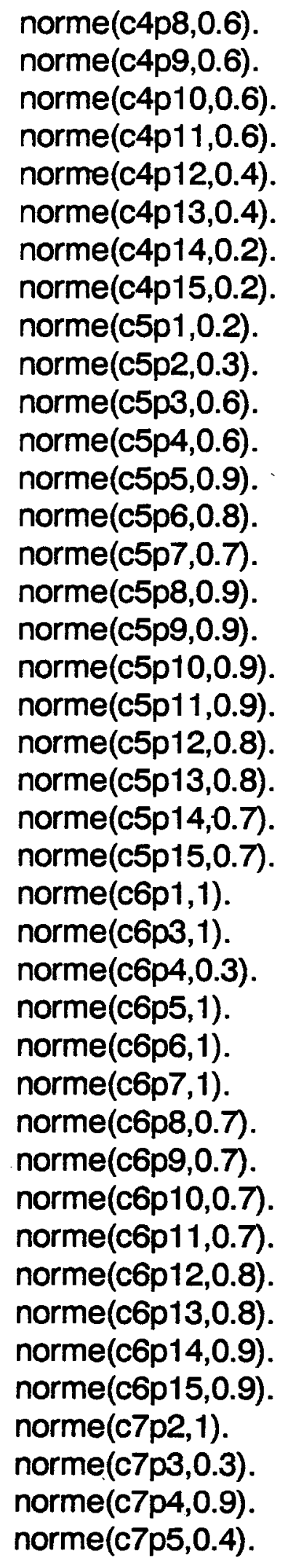




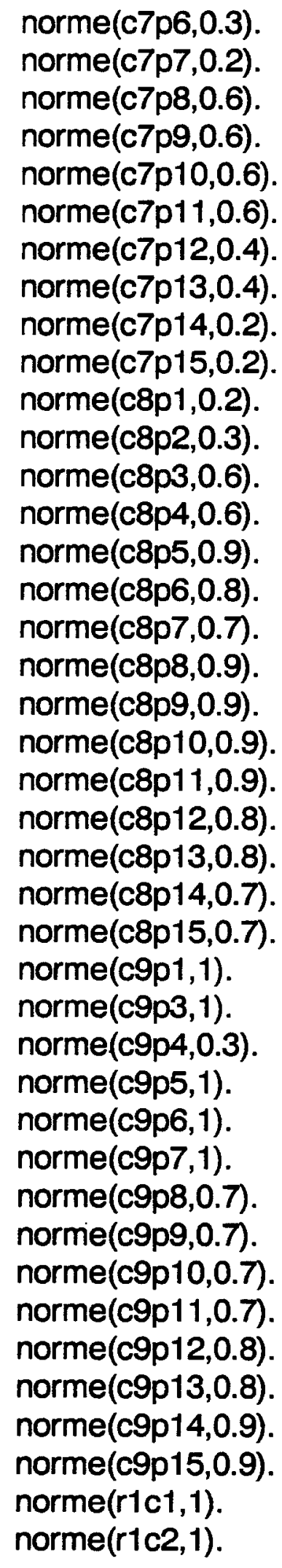


norme $(r 1 c 3,1)$. norme $(r 1 c 4,1)$. norme $(r 1 c 5,1)$. norme $(r 1 c 6,1)$. norme $(r 1 c 7,1)$. norme $(\mathrm{r} 1 \mathrm{c} 8,1)$. norme $(r 1 c 9,1)$. norme $(r 2 c 1,1)$. norme $(r 2 c 2,1)$. norme $(r 2 c 3,1)$. norme $(r 2 c 4,1)$. norme $(r 2 c 5,1)$. norme $(r 2 c 6,1)$. norme $(r 2 c 7,1)$. norme $(r 2 c 8,1)$. norme $(r 2 c 9,1)$. norme $(r 3 c 1,1)$. norme $(r 3 c 2,1)$. norme $\left(r 3 c_{3}, 1\right)$. norme $(r 3 c 4,1)$. norme $(r 3 c 5,1)$. norme $(r 3 c 6,1)$. norme $(r 3 c 7,1)$. norme $(r 3 c 8,1)$. norme $(r 3 c 9,1)$. norme $(r 4 c 1,1)$. norme $(r 4 c 2,1)$. norme $(r 4 c 3,1)$. norme $(r 4 c 4,1)$. norme $(r 4 c 5,1)$. norme $(r 4 c 6,1)$. norme $(r 4 c 7,1)$. norme $(r 4 c 8,1)$. norme $(r 4 c 9,1)$. norme $(r 5 c 1,1)$. norme $(r 5 c 2,1)$. norme $(r 5 c 3,1)$. norme $(r 5 c 4,1)$. norme $(r 5 c 5,1)$. norme $(r 5 c 6,1)$. norme $(r 5 c 7,1)$. 
norme $(r 5 c 8,1)$.

norme $(r 5 c 9,1)$.

norme $(r 6 c 1,1)$.

norme $(r 6 c 2,1)$.

norme $(r 6 c 3,1)$.

norme $(r 6 c 4,1)$.

norme $(\mathrm{r} 6 \mathrm{c} 5,1)$.

norme $(r 6 c 6,1)$.

norme $(r 6 c 7,1)$.

norme $(\mathrm{r} 6 \mathrm{c} 8,1)$.

norme $(r 6 c 9,1)$.

norme $(r 7 c 1,1)$.

norme $(r 7 c 2,1)$.

norme $(r 7 c 3,1)$.

norme $(r 7 c 4,1)$.

norme $(r 7 c 5,1)$.

norme $(r 7 c 6,1)$.

norme $(r 7 c 7,1)$.

norme $(r 7 c 8,1)$.

norme $(r 7 c 9,1)$.

norme $(r 8 c 1,1)$.

norme $(r 8 c 2,1)$.

norme $(r 8 c 3,1)$.

norme $(r 8 c 4,1)$.

norme $(r 8 c 5,1)$.

norme $(r 8 c 6,1)$.

norme $(r 8 c 7,1)$.

norme $(r 8 c 8,1)$.

norme $(r 8 c 9,1)$.

norme $(\mathrm{c} 1 \mathrm{r1}, 0.6)$.

norme $(c 1 r 2,0.5)$.

norme $(c 1 r 3,0.4)$.

norme (c1r4,0.3).

norme (c1r5,0.2).

norme $(c 1 r 6,0.1)$.

norme (c1r8,0.8).

norme $(c 2 r 1,0.8)$.

norme (c2r2,0.7).

norme (c2r3,0.6).

norme $(c 2 r 4,0.5)$.

norme(c2r5,0.4). 
norme (c2r6,0.3).

norme $(c 2 r 7,0.2)$.

norme (c2r8,0.9).

norme $(c 3 r 1,1)$.

norme (c3r2,1).

norme $(333,1)$.

norme $($ c3r 4,1$)$.

norme (c3r5,1).

norme $(c 3 r 6,1)$.

norme $(c 3 r 7,1)$.

norme $(c 3 r 8,1)$.

norme $(c 4 r 1,0.6)$.

norme $(c 4 r 2,0.5)$.

norme $(c 4 r 3,0.4)$.

norme (c4r4,0.3).

norme (c4r5,0.2).

norme $(\mathrm{c} 4 \mathrm{r} 6,0.1)$.

norme (c4r8,0.8).

norme (c5r1,0.8).

norme (c5r2,0.7).

norme (c5r3,0.6).

norme (c5r4,0.5).

norme (c5r5,0.4).

norme (c5r6,0.3).

norme (c5r7,0.2).

norme (c5r8,0.9).

norme (c6r1,1).

norme $(c 6 r 2,1)$.

norme (c6r3,1).

norme (c6r4,1).

norme (c6r5,1).

norme (c6r6,1).

norme $(c 6 r 7,1)$.

norme (c6r8,1).

norme $(\mathrm{c} 7 \mathrm{rt}, 0.6)$.

norme $(c 7 r 2,0.5)$.

norme $(c 7 r 3,0.4)$.

norme $(c 7 r 4,0.3)$.

norme (c7r5,0.2).

norme $(c 7 r 6,0.1)$.

norme $(c 7 r 8,0.8)$. 
norme (c8r1, 0.8).

norme (c8r2,0.7).

norme (c8r3,0.6).

norme $(c 8 r 4,0.5)$.

norme (c8r5,0.4).

norme (c8r6,0.3).

norme $(c 8 r 7,0.2)$.

norme $(c 8 r 8,0.9)$.

norme (c9r1,1).

norme(c9r2,1).

norme (c9r3,1).

norme (c9r4,1).

norme (c9r5,1).

norme (c9r6,1).

norme (c9r7,1).

norme (c9r8,1).

norme $(p 1 r 1,0.8)$.

norme $(p 1 \mathrm{r} 2,0.8)$.

norme $(p 1 r 3,0.8)$.

norme (p1r4,0.8).

norme $(p 1 r 5,0.8)$.

norme (p1r6,1).

norme (p2r1,0.7).

norme (p2r2,0.7).

norme (p2r3,0.7).

norme (p2r4,0.7).

norme (p2r8,1).

norme (p3r1,0.4).

norme $(p 3 r 2,0.4)$.

norme $(p 3 r 3,0.4)$.

norme (p3r4,1).

norme (p3r5,1).

norme $(p 3 r 6,0.9)$.

norme (p3r8,0.75).

norme $(p 4 r 8,0.8)$.

norme (p5r1,0.7).

norme (p5r2,0.7).

norme $(p 5 r 5,0.6)$.

norme (p5r6,0.8).

norme (p6r1,0.6).

norme(p6r2,0.6). 
norme (p6r3,0.7).

norme (p6r5,0.6).

norme (p6r6,0.8).

norme $(p 7 r 3,0.6)$.

norme (p7r4,0.7).

norme (p7r5,0.6).

norme (p7r6,0.8).

norme (p8r1,1).

norme (p8r5,0.3).

norme (p9r1,1).

norme (p9r5,0.3).

norme (p10r1,0.9).

norme (p10r2,1).

norme(p10r5,0.3).

norme (p11r1,0.9).

norme (p11r2,1).

norme (p11r5,0.3).

norme (p12r2,0.9).

norme (p12r3,1).

norme (p12r5,0.3).

norme (p13r2,0.9).

norme (p13r3,1).

norme (p13r5,0.3).

norme (p14r3,0.9).

norme (p14r4,1).

norme (p14r5,0.3).

norme (p15r3,0.9).

norme (p15r4,1).

norme (p15r5,0.3).

norme(r1a1,0).

norme(r1a2,1).

norme $(r 1 a 3,1)$.

norme $(r 1 a 4,1)$.

norme(r1a5,1).

norme $(r 1 a 7,1)$.

norme (r1a8,1).

norme $(r 1 a 9,1)$.

norme(r1a13,1).

norme $(r 1 a 17,1)$.

norme $(r 1 a 18,0.9)$.

norme(r1a22,0.8). 
norme $(r 1 a 23,0.8)$.

norme $(r 1230,1)$.

norme $(r 2 a 1,0)$.

norme (r2a2,1).

norme (r2a3,1).

norme (r2a4,1).

norme(r2a6,1).

norme $(r 2 a 7,1)$.

norme $(r 2 a 10,1)$.

norme(r2a11,1).

norme $(r 2 a 14,1)$.

norme(r2a17,1).

norme (r2a19,0.9).

norme (r2a24,0.8).

norme (r2a25,0.8).

norme $(r 2 a 30,1)$.

norme $(r 3 a 1,0)$.

norme $(r 3 a 2,1)$.

norme $(r 3 a 3,1)$.

norme $($ ß3a5,1).

norme $(r 3 a 6,1)$.

norme $(r 3 a 8,1)$.

norme $($ (3a10,1).

norme(r3a12,1).

norme(r3a15,1).

norme $(r 3 a 17,1)$.

norme $($ r3a20,0.9).

norme $($ (3a26,0.8).

norme $(r 3 a 27,0.8)$.

norme $($ r $3230,0.9)$.

norme $(r 4 a 1,0)$.

norme $(r 4 a 2,1)$.

norme $(r 4 a 4,1)$.

norme $(r 4 a 5,1)$.

norme $(r 4 a 6,1)$.

norme $(r 4 a 9,1)$.

norme(r4a11,1).

norme (r4a12,1).

norme $(r 4 a 16,1)$.

norme $(r 4 a 17,1)$.

norme $(r 4 a 21,0.9)$. 


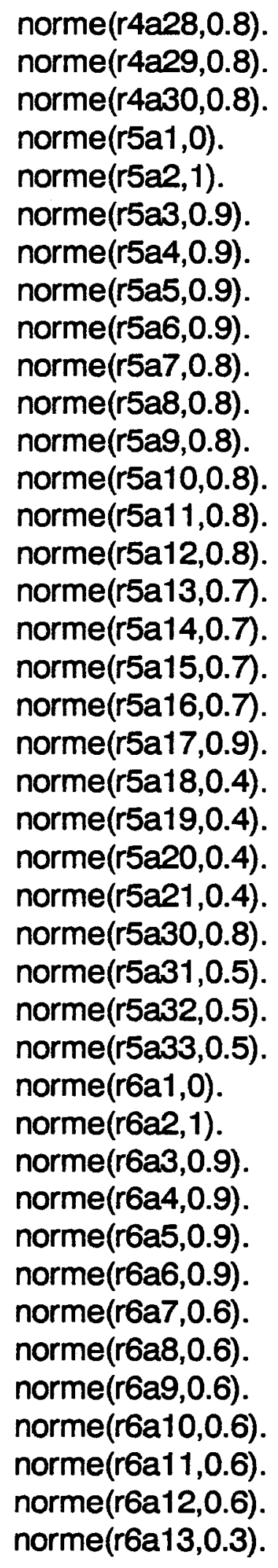


norme(r6a14,0.3). norme(r6a15,0.3). norme(r6a16,0.3). norme(r6a17,0.7). norme (r6a30,0.5). norme $(r 7 a 1,1)$. norme $(r 8 a 1,0)$. norme $(r 8 a 2,1)$. norme $(r 8 a 3,1)$. norme $(r 8 a 4,1)$. norme $(r 8 a 5,1)$. norme $(r 8 a 6,1)$. norme $(r 8 a 7,1)$. norme $(r 8 a 8,1)$. norme $(r 8 a 9,1)$. norme (r8a10,1). norme(r8a11,1). norme (r8a12,1). norme (r8a13,1). norme(r8a14,1). norme (r8a15,1). norme(r8a16,1). norme $($ r8a17,1). norme(r8a18,1). norme $($ r8a19,1). norme $(r 8 a 20,1)$. norme $(r 8 a 21,1)$. norme (r8a22,1). norme (r8a23,1). norme (r8a24,1). norme (r8a25,1). norme (r8a26,1). norme $(r 8 a 27,1)$. norme (r8a28,1). norme $(r 8 a 29,1)$. norme $(r 8 a 30,1)$. norme (r8a31,0.9). norme (r8a32,0.9). norme $(r 8 a 33,0.9)$. norme(Xiyj,0). 


\section{PARTIE 3}

\section{LES CONTENUS DES BASES DE CONNAISSANCES}




\section{PARTIE 3}

\section{LES CONTENUS DES BASES DE CONNAISSANCES}

\section{1: La base Programme:}

P1: Simplification d'une expression booléenne donnée

P2: Introduction à l'algèbre de Boole

P3: Simplification d'expressions booléennes

P4: Ensemble des postulats

P5: Les théorèmes de niveau 1

P6: Les théorèmes de niveau 2

P7: Les théorèmes de niveau 3

P8: Théorème de l'élément neutre du produit logique

P9: Théorème de l'élément neutre de la somme logique

P10: Théorème de la complémentation du produit logique

P11: Théorème de la complémentation de la somme logique

P12: Théorème de la distributivité du produit sur la somme logique

P13: Théorème de la distributivité de la somme sur le produit logique

P14: La première de De Morgan

P15: La deuxième loi de De Morgan 


\section{2: La base Contexte:}

C1: L'apprenant est visuel et lent

C2: L'apprenant est visuel et de vitesse moyenne

C3: L'apprenant est visuel et rapide

C4: L'apprenant est auditif et lent

C5: L'apprenant est auditif et de vitesse moyenne

C6: L'appreannt est auditif et rapide

C7: L'apprenant est kinesthésique et lent

C8: L'apprenant est kinesthésique et de vitesse moyenne

C9: L'apprenant est kinesthésique et rapide 


\section{3: La base Activité:}

A1: Simplifier l'expression donnée

A2: Simplifier des expressions booléennes concernant l'ensemble des théorèmes

A3: Simplifier des expressions booléennes concernant les théorèmes de l'élément neutre, complémentation et distributivité

A4: Simplifier des expressions booléennes concernant les théorèmes de l'élément neutre, complémentation et De Morgan

A5: Simplifier des expressions booléennes concernant les théorèmes de l'élément neutre, distributivité et De Morgan

A6: Simplifier des expressions booléennes concernant les théorèmes de complémentation, distrubutivité et De Morgan

A7: Simplifier des expressions booléennes concernant les théorèmes d'élément neutre et complémentation

A8: Simplifier des expressions booléennes concernant les théorèmes d'élément neutre et distributivité

A9: Simplifier des expressions booléennes concernant les théorèmes d'élément neutre et De Morgan

A10: Simplifier des expressions booléennes concernant les théorèmes de complémentation et distributivité

A11: Simplifier des expressions booléennes concernant les théorèmes de complémentation et De Morgan

A12: Simplifier des expressions booléennes concernant les théorèmes de distributivité et De Morgan

A13: Simplifier des expressions booléennes concernant les théorèmes de l'élément neutre

A14: Simplifier des expressions booléennes concernant les théorèmes de la complémentation

A15: Simplifier des expressions booléennes concernant les théorèmes de la distributivité 
A16: Simplifier des expressions booléennes concernant les lois de De Morgan

A17: Compléter des énoncés de théorèmes

A18: Compléter des tables de vérité concernant les théorèmes de l'élément neutre

A19: Compléter des tables de vérité concernant les théorèmes de la complémentation

A20: Compléter des tables de vérité concernant les théorèmes de la distributivité

A21: Compléter des tables de vérité concernant les lois de De Morgan

A22: Résoudre des expressions booléennes à l'aide de l'élément neutre du produit logique

A23: Résoudre des expressions booléennes à l'aide de l'élément neutre de la somme logique

A24: Résoudre des expressions booléennes à l'aide de la complémentation du produit logique

A25: Résoudre des expressions booléennes à l'aide de la complémentation de la somme logique

A26: Résoudre des expressions booléennes à l'aide de la distributivité du produit sur la somme logique

A27: Résoudre des expressions booléennes à l'aide de la distributivité de la somme sur le produit logique

A28: Résoudre des expressions booléennes à l'aide de la première loi de De Morgan

A29: Résoudre des expressions booléennes à l'aide de la deuxième loi de De Morgan

A30: Compléter des tables de vérité concernant l'ensemble des postulats

A31: Répondre à certaines questions concernant l'ensemble des postulats présentés à des apprenants visuels

A32: Répondre à certaines questions concernant l'ensemble des postulats présentés à des apprenants auditifs

A33: Répondre à certaines questions concernant l'ensemble des postulats présentés à des apprenants kinesthésiques 


\section{4: La base Résultat:}

R1: Utilisation des théorèmes de l'élément neutre

R2: Utilisation des théorèmes de la complémentation

R3: Utilisation des théorèmes de la distributivité

R4: Utilisation des lois de De Morgan

R5: Simplification d'expressions booléennes à 2 variables

R6: Simplification d'expressions booléennes à 3 variables

R7: Simplification de l'expression booléenne prédéfinie

R8: Utilisation des postulats 


\section{PARTIE 4}

\section{LE CAHIER D'ACTIVITES DE PROLIN}




\section{PARTIE 4}

\section{LE CAHIER D'ACTIVITES DE PROLIN}

Le cahier qui suit constitue l'outil de travail de l'apprenant lors de l'apprentissage. Les activités sont numérotées de façon à correspondre aux numéros d'activité que génère PROLIN. Ainsi, l'apprenant qui utilise PROLIN, n'a qu'à consulter le présent cahier pour faire les exercices que demande PROLIN. Les notes demandées par PROLIN sont obtenues en additionnant les codes de résultat correspondant à chacun des choix de réponse comme indiqué dans le cahier.

La situation idéale serait d'incorporer le cahier à l'intérieur d'un logiciel qui serait directement lié à PROLIN. Ce dernier ferait alors le calcul des notes luimême.

Les simulations ont été faites en tenant compte de ces activités en tentant d'immiter les comportements d'apprenants dans toutes sortes de situations.

Veuillez noter qu'il paraît évident qu'on ne pourrait se servir directement de ce cahier dans une vraie situation d'apprentissage. Nous sommes bien conscient que la présentation des choix de réponses suggère les réponse puisque la meilleure réponse possède le code de résultat le plus élevé. 


\section{Activité 1}

Simplifier l'expression booléenne suivante:

$$
A B \bar{C}+A B C+C((A+B)(\overline{A \bar{B}})
$$

Choix de réponses:
A B
$->$
0.7
$C B$
$->$
0.7
$(A+C) B$
$->$
1.0
$A B \bar{C}+C$
$->$
0.0
$A C+B$
$->$
0.8

Entrer au clavier le nombre (à droite) correspondant à la réponse obtenue. 


\section{Activité 2}

Simplifier les expressions booléennes suivantes:

a) $A(\overline{A \bar{B}})$

Choix de réponses:

$\begin{array}{lll}A+B & -> & 0.0 \\ A \bar{B} & -> & 0.1 \\ A B & -> & 0.3 \\ 1 & -> & 0.0\end{array}$

b) $A+(\overline{A+\bar{B}})$

Choix de réponses:

$\begin{array}{lll}A+B & -> & 0.3 \\ A \bar{B} & -> & 0.1 \\ A B & -> & 0.0 \\ 1 & -> & 0.0\end{array}$

c) $C[(A+B)(\overline{A \bar{B}})]$

Choix de réponses:

$\begin{array}{lll}\text { C }(A+B) & --> & 0.0 \\ \text { C A B } & -> & 0.1 \\ \text { C B } & -> & 0.4 \\ \text { C A } & -> & 0.2\end{array}$

Entrer au clavier la somme des nombres correspondants aux réponses obtenues. 


\section{Activité 3}

Simplifier les expressions booléennes suivantes:
a) $A B C+A B \bar{C}$

Choix de réponses:

$\begin{array}{lll}A & --> & 0.0 \\ A B & --> & 0.3 \\ A B C & --> & 0.0 \\ A B \bar{C} & --> & 0.0\end{array}$

b) $(A+B)(\bar{A}+B)$

Choix de réponses:

$\begin{array}{lll}\text { A } & -> & 0.0 \\ \text { B } & -> & 0.4 \\ \text { A B } & -> & 0.0 \\ \bar{A} \text { B } & --> & 0.0\end{array}$

c) $A B C+A \overline{B C}$

Choix de réponses:

$\begin{array}{lll}A & -> & 0.3 \\ A B & -> & 0.0 \\ A B C & -> & 0.0 \\ A B \bar{C} & -> & 0.0\end{array}$

Entrer au clavier la somme des nombres correspondants aux réponses obtenues. 


\section{Activité 4}

Simplifier les expressions booléennes suivantes:

a) $(A+\bar{A}) B$

Choix de réponses:

$\begin{array}{lll}\text { A } & --> & 0.0 \\ \text { A } & -> & 0.0 \\ \text { B } & -> & 0.3 \\ \text { A B } & -> & 0.0\end{array}$

b) $A(B \bar{B}+C)$

Choix de réponses:

$\begin{array}{lll}A C & -> & 0.4 \\ A+C & -> & 0.0 \\ A B+C & -> & 0.0 \\ A B+\bar{B}+C & -> & 0.0\end{array}$

c) $A+(B \bar{B}+C)$

Choix de réponses:

$\begin{array}{lll}A C & -> & 0.0 \\ A+C & -> & 0.3 \\ A B+C & -> & 0.0 \\ A B+\bar{B}+C & -> & 0.0\end{array}$

Entrer au clavier la somme des nombres correspondants aux réponses obtenues. 


\section{Activité 5}

Simplifier les expressions booléennes suivantes:

a) $A B C+A B$

Choix de réponses:

$\begin{array}{lll}A B+(C 1) & -> & 0.0 \\ A B+(C+1) & --> & 0.0 \\ A B(C+1) & -\rightarrow & 0.3 \\ A B(C 1) & -> & 0.0\end{array}$

b) $(A+B)(\overline{A \bar{B}})$

Choix de réponses:

$\begin{array}{lll}(A+\bar{A}) B & -\rightarrow & 0.0 \\ A \bar{A}+B & -\rightarrow & 0.4 \\ A(A+B) & -> & 0.0 \\ A+(\bar{A} B) & -> & 0.0\end{array}$

c) $A B+A$

Choix de réponses:
$A B$
$->$
0.0
$A+(B+A)$
$\rightarrow$
0.0
$A+B$
$\rightarrow$
0.0
A
$->$
0.3

Entrer au clavier la somme des nombres correspondants aux réponses obtenues. 


\section{Activité 6}

Simplifier les expressions booléennes suivantes:

a) $A(\overline{A \bar{B}})$

Choix de réponses:

$\begin{array}{lll}\bar{A}+A B & -> & 0.0 \\ 0+A B & -> & 0.3 \\ 1+A B & -> & 0.0 \\ A \bar{A}+B & -> & 0.0\end{array}$

b) $A+(\overline{A+\bar{B}})$

Choix de réponses:

$\begin{array}{lll}1(A+B) & -> & 0.4 \\ 0(A+B) & -> & 0.0 \\ (A+\bar{A}) 1 & -> & 0.0 \\ (A+\bar{A}) 0 & -> & 0.0\end{array}$

c) $(A+B)(\overline{A \bar{B}})$

Choix de réponses:

$\begin{array}{lll}\bar{A}+B & -> & 0.0 \\ A+B & -> & 0.0 \\ 1+B & -> & 0.0 \\ 0+B & -> & 0.3\end{array}$

Entrer au clavier la somme des nombres correspondants aux réponses obtenues. 


\section{Activité 7}

Simplifier les expressions booléennes suivantes:

a) $A \bar{A}+B$

Choix de réponses:

$\begin{array}{lll}A & -> & 0.0 \\ B & -> & 0.3 \\ A+B & -> & 0.0 \\ \text { A B } & -> & 0.0\end{array}$

b) $A B(\bar{C}+C)$

Choix de réponses:

$\begin{array}{lll}A B & -> & 0.4 \\ A B+C & -> & 0.0 \\ 1 & -> & 0.0 \\ A B C & -> & 0.0\end{array}$

c) $A B(\bar{C}+C)$

Choix de réponses:
$A B$
$->$
0.0
$A B+C$
0.0
1
$\begin{array}{ll}--> & 0.0 \\ --> & 0.3\end{array}$
A B C
0.0

Entrer au clavier la somme des nombres correspondants aux réponses obtenues. 


\section{Activité 8}

Simplifier les expressions booléennes suivantes:

a) $A B+A$

Choix de réponses:

$\begin{array}{lll}A(B+0) & -> & 0.0 \\ A(B+1) & -> & 0.3 \\ (A+B) A & -> & 0.0 \\ A+(B 0) & -> & 0.0\end{array}$

b) $A B C+A B$

Choix de réponses:

$\begin{array}{lll}A B & -> & 0.35 \\ A B C & -> & 0.0 \\ A B+C & -> & 0.0 \\ A+B C & -> & 0.0\end{array}$

c) $A(B+B C)$

Choix de réponses:
$A B+C$
$\rightarrow$
0.0
$A+B C$
$->$
$A B$
$->$
0.0
$A C$
$->$
0.35
0.0

Entrer au clavier la somme des nombres correspondants aux réponses obtenues. 


\section{Activité 9}

Simplifier les expressions booléennes suivantes:

a) $0+(A+B)$

Choix de réponses:

$\begin{array}{lll}0 & -> & 0.0 \\ 1 & -> & 0.0 \\ A+B & -> & 0.3 \\ A B & -> & 0.0\end{array}$

b) $(A+B)(\overline{A \bar{B}})$

Choix de réponses:

$$
\begin{array}{lll}
(A+B)(A+\bar{B}) & -> & 0.1 \\
(A+B)(\bar{A} B) & -> & 0.0 \\
(A+B)(A \bar{B}) & -> & 0.0 \\
(A+B)(\bar{A}+B) & -> & 0.4
\end{array}
$$

c) $A B C+A B$

Choix de réponses:

$\begin{array}{lll}A B C+A B 1 & -> & 0.3 \\ A B C+A B 0 & --> & 0.0 \\ A B C+A B+A & -> & 0.0 \\ A B C+\bar{A} \bar{B} & --> & 0.0\end{array}$

Entrer au clavier la somme des nombres correspondants aux réponses obtenues. 
Activité 10

Simplifier les expressions booléennes suivantes:

a) $A B C+A B \bar{C}$

Choix de réponses:

$\begin{array}{lll}A B(0) & -> & 0.1 \\ A B C+0 & -> & 0.0 \\ A B(1) & -> & 0.3 \\ A B C+1 & -> & 0.0\end{array}$

b) $(A+B)(\bar{A}+B)$

Choix de réponses:

$\begin{array}{lll}0+B & -> & 0.3 \\ O B & --> & 0.1 \\ 1+B & --> & 0.1 \\ A B & -> & 0.0\end{array}$

c) $A B C+A \overline{B C}$

Choix de réponses:
$A(0)$
$->$
0.1
$A(1)$
$->$
0.4
$A B+\bar{C}$
$->$
0.0
$0+(B C+\overline{B C})$
$->$
0.0

Entrer au clavier la somme des nombres correspondants aux réponses obtenues. 


\section{Activité 11}

Simplifier les expressions booléennes suivantes:

a) $\overline{A B}+A B$

Choix de réponses:

\begin{tabular}{lll} 
A B & $->$ & 0.0 \\
0 & $->$ & 0.0 \\
1 & $-\rightarrow$ & 0.3 \\
\hline A B & $->$ & 0.0
\end{tabular}

b) $(A+B)(\overline{A+B})$

Choix de réponses:

$\begin{array}{lll}A+B & -> & 0.0 \\ 0 & -> & 0.3 \\ \frac{1}{A}+\bar{B} & -> & 0.0 \\ & -> & 0.0\end{array}$

c) $\overline{A B \bar{C}}$

Choix de réponses:

$\begin{array}{lll}\bar{A}+\bar{B}+C & -> & 0.4 \\ \bar{A} \bar{B} C & --> & 0.0 \\ \overline{A B}+\bar{C} & -> & 0.0 \\ A B+\bar{C} & -> & 0.0\end{array}$

Entrer au clavier la somme des nombres correspondants aux réponses obtenues. 


\section{Activité 12}

Simplifier les expressions booléennes suivantes:

a) $A(\overline{A \bar{B}})$

Choix de réponses:

$\begin{array}{lll}A B+\bar{A} B & -> & 0.1 \\ A+\bar{A} B & -> & 0.0 \\ A \bar{A}+B & \rightarrow> & 0.0 \\ A \bar{A}+A B & -> & 0.3\end{array}$

b) $A+(\overline{A+\bar{B}})$

Choix de réponses:

$\begin{array}{lll}A+\bar{A}+B & -> & 0.0 \\ (A+\bar{A})(A+B) & -> & 0.3 \\ A \bar{A}+B & -> & 0.0 \\ A \bar{A}+A B & -> & 0.0\end{array}$

c) $C[(A+B)(\overline{A \bar{B}})]$

Choix de réponses:

$\begin{array}{lll}C[A \bar{A}+B] & -> & 0.4 \\ C[(A+\bar{A}) B] & --> & 0.0 \\ C[A(A+B)] & -\rightarrow & 0.0 \\ C[A+(\bar{A} B)] & -\rightarrow & 0.0\end{array}$

Entrer au clavier la somme des nombres correspondants aux réponses obtenues. 


\section{Activité 13}

Simplifier les expressions booléennes suivantes:

a) $A+B+0$

Choix de réponses:

$\begin{array}{lll}0 & -> & 0.0 \\ 1 & -> & 0.0 \\ A+B & \rightarrow & 0.3 \\ A B & \rightarrow & 0.0\end{array}$

b) $1(A+B)$

Choix de réponses:

$\begin{array}{lll}0 & -> & 0.0 \\ 1 & -> & 0.0 \\ A+B & -> & 0.4 \\ A B & -> & 0.0\end{array}$

c) $A 1 B$

Choix de réponses:

$\begin{array}{lll}0 & -> & 0.0 \\ 1 & -> & 0.0 \\ A B & -> & 0.3 \\ A+B & -> & 0.0\end{array}$

Entrer au clavier la somme des nombres correspondants aux réponses obtenues. 


\section{Activité 14}

Simplifier les expressions booléennes suivantes:

a) $A+\bar{A}$

Choix de réponses:

$\begin{array}{lll}0 & -> & 0.0 \\ 1 & -> & 0.2 \\ \text { A } & -> & 0.0 \\ \text { A } & -> & 0.0\end{array}$

b) $B \bar{B}$

Choix de réponses:

\begin{tabular}{lll}
0 & $->$ & 0.2 \\
1 & $-->$ & 0.0 \\
B & $->$ & 0.0 \\
\hline B & $->$ & 0.0
\end{tabular}

c) $A B(\bar{C}+C)$

Choix de réponses:

$\begin{array}{lll}A B 0 & -> & 0.2 \\ \text { AB } 1 & -> & 0.6 \\ \text { ABC } & -\rightarrow & 0.0 \\ \text { AB C } & -> & 0.0\end{array}$

Entrer au clavier la somme des nombres correspondants aux réponses obtenues. 
Activité 15

Simplifier les expressions booléennes suivantes:

a) $A B+A C$

Choix de réponses:

$\begin{array}{lll}A B C & -> & 0.0 \\ A(B+C) & --> & 0.3 \\ B C & --> & 0.0 \\ A B+C & -> & 0.0\end{array}$

b) $A B C+A B \bar{C}$

Choix de réponses:

$\begin{array}{lll}A B C+\bar{C} & -> & 0.0 \\ A B(C+\bar{C}) & -> & 0.3 \\ A B+C \bar{C} & -> & 0.0 \\ (A+B+C) \bar{C} & -> & 0.0\end{array}$

c) $(A+B)(\bar{A}+B)$

Choix de réponses:

$\begin{array}{lll}B(\bar{A}+B) & -> & 0.0 \\ (A+\bar{A}) B & -> & 0.0 \\ A \bar{A}+B & -> & 0.4 \\ A(\bar{A}+B) & -> & 0.0\end{array}$

Entrer au clavier la somme des nombres correspondants aux réponses obtenues. 


\section{Activité 16}

Simplifier les expressions booléennes suivantes:

a) $\overline{A+\bar{B}}$

Choix de réponses:

$\begin{array}{lll}\bar{A} B & -> & 0.3 \\ \bar{A}+B & -> & 0.1 \\ A \bar{B} & -> & 0.1 \\ A B & -> & 0.0\end{array}$

b) $A(\overline{A \bar{B}})$

Choix de réponses:
$A(\bar{A} B)$
$->$
0.1
$A(\bar{A}+B)$
$->$
0.4
$A(A \bar{B})$
$->$
0.0
$A(A+\bar{B})$
$\rightarrow$
0.1

c) $A(\overline{A+\bar{B}})$

Choix de réponses:
$A+(A+\bar{B})$
$->$
0.0
$A+(\bar{A}+B)$
0.1
$A+(A \bar{B})$
$->$
0.1
$A+(\bar{A} B)$
->>
0.3

Entrer au clavier la somme des nombres correspondants aux réponses obtenues. 


\section{Activité 17}

Pour toutes variables de Boole $\mathrm{A}, \mathrm{B}$ et $\mathrm{C}$ quelconques, il existe un certain nombre de théorèmes qui nous aident à simplifier des expressions booléennes.

Compléter les théorèmes qui suivent en choisissant la bonne réponse parmi celles qui sont proposées.

a) Elément neutre du produit logique:

Pour toute proposition $A$, on a
$A O=A$
$->$
0.0
$A 1=A$
$->$
0.1

b) Elément neutre de la somme logique:

Pour toute proposition $A$, on a

$$
\begin{aligned}
& A+0=A \\
& -> \\
& A+1=A \\
& ->
\end{aligned}
$$

c) Complémentation du produit logique:

Pour toute proposition $A$, on a
$A \bar{A}=0$
$-->$
0.1
$A \bar{A}=1$
$-->$
0.0

$*$

d) Complémentation de la somme logique:

Pour toute proposition $A$, on a

$$
\begin{array}{lll}
\bar{A}+A=0 & -\rightarrow & 0.0 \\
\bar{A}+A=1 & -> & 0.1
\end{array}
$$

e) Distributivité de l'addition sur le produit logique:

Pour toutes propositions $A, B$ et $C$, on a
$A+(B C)=A B+A C$
$\rightarrow \quad 0.0$
$A+(B C)=(A+B)(A+C)$
$\begin{array}{ll}-> & 0.15\end{array}$
$A+(B C)=(A+B)(B+C)$
$\rightarrow \quad 0.0$
$A+(B C)=A(B+C)$
$\rightarrow \quad 0.0$ 
Activité 17 (suite)

f) Distributivité du produit sur la somme logique:

Pour toutes propositions $A, B$ et $C$, on a
$A B+A C=A+(B C)$
$\rightarrow \quad 0.0$
$A B+A C=A(B+C)$
$->$
0.15
$A B+A C=(A+B)(A+C)$
$->$
0.0
$A B+A C=A B+B C$
$->$
0.0

g) Première loi de De Morgan:

Pour toutes propositions $A$ et $B$, on a

$\begin{array}{llll}\overline{A B} & =\bar{A} \bar{B} & \rightarrow & 0.0 \\ \overline{A B}=\bar{A} B & \rightarrow & 0.0 \\ \overline{A B}=\bar{A}+\bar{B} & \rightarrow & 0.15 \\ \overline{A B}=A+B & \rightarrow & 0.0\end{array}$

h) Deuxième loi de De Morgan:

Pour toutes propositions $A$ et $B$, on a

$$
\begin{array}{lll}
\overline{A+B}=\bar{A} \bar{B} & -> & 0.15 \\
\overline{A+B}=\bar{A} B & -> & 0.0 \\
\overline{A+B}=\bar{A}+\bar{B} & -> & 0.0 \\
\overline{A+B}=A+B & -> & 0.0
\end{array}
$$

Entrer au clavier la somme des nombres correspondants aux réponses obtenues. 


\section{Activité 18}

Compléter les tables de vérités qui suivent:

\begin{tabular}{c|c}
$A$ & $1 \cdot A$ \\
\hline 0 & 0 \\
1 & 1
\end{tabular}

\begin{tabular}{c|c}
$A$ & $A \cdot 1$ \\
\hline 0 & $a$ \\
1 & $b$
\end{tabular}

\begin{tabular}{c|c}
$A$ & $0+A$ \\
\hline 0 & 0 \\
1 & $C$
\end{tabular}

\begin{tabular}{c|c}
$A$ & $A+0$ \\
\hline 0 & $d$ \\
1 & $e$
\end{tabular}

Choix de réponses:

\begin{tabular}{|c|c|c|c|}
\hline \multirow[t]{2}{*}{$a=$} & 0 & $->$ & 0.2 \\
\hline & 1 & $-\rightarrow$ & 0.0 \\
\hline \multirow[t]{2}{*}{$b=$} & 0 & $-\rightarrow$ & \\
\hline & 1 & $-\rightarrow$ & \\
\hline \multirow[t]{2}{*}{$C=$} & 0 & $-\rightarrow$ & \\
\hline & 1 & $-\rightarrow$ & \\
\hline \multirow[t]{2}{*}{$d=$} & 0 & $-->$ & \\
\hline & 1 & $-\rightarrow$ & \\
\hline \multirow[t]{2}{*}{$e=$} & 0 & $->$ & \\
\hline & 1 & $-\rightarrow$ & \\
\hline
\end{tabular}

Entrer au clavier la somme des nombres correspondants aux réponses obtenues. 
Activité 19

Compléter les tables de vérités qui suivent:

\begin{tabular}{c|c}
$A$ & $A \cdot \bar{A}$ \\
\hline 0 & 0 \\
1 & $a$
\end{tabular}

\begin{tabular}{c|c}
$A$ & $\bar{A} \cdot A$ \\
\hline 0 & 0 \\
1 & $b$
\end{tabular}

\begin{tabular}{c|c}
$A$ & $A+\bar{A}$ \\
\hline 0 & $c$ \\
1 & $d$
\end{tabular}

\begin{tabular}{c|c}
$A$ & $\bar{A}+A$ \\
\hline 0 & 1 \\
1 & $e$
\end{tabular}

Choix de réponses:

\begin{tabular}{|c|c|c|c|}
\hline$a=$ & 0 & $-\rightarrow$ & 0.2 \\
\hline & 1 & $\rightarrow$ & 0.0 \\
\hline$b=$ & 0 & $-\rightarrow$ & 0.2 \\
\hline & 1 & $\rightarrow>$ & 0.0 \\
\hline$c=$ & 0 & $\rightarrow>$ & 0.0 \\
\hline & 1 & $\rightarrow>$ & 0.2 \\
\hline$d=$ & 0 & $\Rightarrow$ & 0.0 \\
\hline & 1 & $-\rightarrow$ & 0.2 \\
\hline$e=$ & 0 & $-->$ & 0.0 \\
\hline & 1 & $-\rightarrow$ & 0.2 \\
\hline
\end{tabular}

Entrer au clavier la somme des nombres correspondants aux réponses obtenues. 


\section{Activité 20}

Compléter les tables de vérités qui suivent:

\begin{tabular}{c|c|c|c|c}
$A$ & $B$ & $C$ & $A+(B C)$ & $(A+B)(A+C)$ \\
\hline 0 & 0 & 0 & 0 & 0 \\
0 & 0 & 1 & $a$ & 0 \\
0 & 1 & 0 & 0 & 0 \\
0 & 1 & 1 & $b$ & 1 \\
1 & 0 & 0 & 1 & $c$ \\
1 & 0 & 1 & 1 & 1 \\
1 & 1 & 0 & 1 & $d$ \\
1 & 1 & 1 & $e$ & 1
\end{tabular}

\begin{tabular}{c|c|c|c|c}
$A$ & $B$ & $C$ & $A(B+C)$ & $(A B)+(A C)$ \\
\hline 0 & 0 & 0 & 0 & 0 \\
0 & 0 & 1 & $f$ & 0 \\
0 & 1 & 0 & 0 & $g$ \\
0 & 1 & 1 & $h$ & 0 \\
1 & 0 & 0 & 0 & 0 \\
1 & 0 & 1 & 1 & 1 \\
1 & 1 & 0 & 1 & $i$ \\
1 & 1 & 1 & $j$ & 1
\end{tabular}


Activité 20 (suite)

Choix de réponses:

\begin{tabular}{|c|c|c|c|}
\hline \multirow[t]{2}{*}{$a=$} & 0 & $->$ & 0.1 \\
\hline & 1 & $->$ & 0.0 \\
\hline \multirow[t]{2}{*}{$b=$} & 0 & $->$ & 0.0 \\
\hline & 1 & $\rightarrow$ & 0.1 \\
\hline \multirow[t]{2}{*}{$c=$} & 0 & $\rightarrow$ & 0.0 \\
\hline & 1 & $\rightarrow$ & 0.1 \\
\hline \multirow[t]{2}{*}{$d=$} & 0 & $-\rightarrow$ & 0.0 \\
\hline & 1 & $-\rightarrow$ & 0.1 \\
\hline \multirow[t]{2}{*}{$e=$} & 0 & $-\rightarrow$ & 0.0 \\
\hline & 1 & $\rightarrow>$ & 0.1 \\
\hline \multirow[t]{2}{*}{$f=$} & 0 & $\rightarrow$ & 0.1 \\
\hline & 1 & $-\rightarrow$ & 0.0 \\
\hline \multirow[t]{2}{*}{$g=$} & 0 & $\rightarrow$ & 0.1 \\
\hline & 1 & $-\rightarrow$ & 0.0 \\
\hline \multirow[t]{2}{*}{$\mathrm{h}=$} & 0 & $-\rightarrow$ & 0.1 \\
\hline & 1 & $\rightarrow$ & 0.0 \\
\hline \multirow[t]{2}{*}{$\mathrm{i}=$} & 0 & $-\rightarrow$ & 0.0 \\
\hline & 1 & $-\rightarrow$ & 0.1 \\
\hline \multirow[t]{2}{*}{$\mathrm{j}=$} & 0 & $\rightarrow>$ & 0.0 \\
\hline & 1 & $-\rightarrow$ & 0.1 \\
\hline
\end{tabular}

Entrer au clavier la somme des nombres correspondants aux réponses obtenues. 
Activité 21

Compléter les tables de vérités qui suivent:

\begin{tabular}{c|c|c|c}
$A$ & $B$ & $\overline{A B}$ & $\bar{A}+\bar{B}$ \\
\hline 0 & 0 & 1 & $a$ \\
0 & 1 & $b$ & 1 \\
1 & 0 & 1 & $c$ \\
1 & 1 & 0 & 0
\end{tabular}

\begin{tabular}{c|c|c|c}
$A$ & $B$ & $\overline{A+B}$ & $\bar{A} \bar{B}$ \\
\hline 0 & 0 & 1 & 1 \\
0 & 1 & $d$ & 0 \\
1 & 0 & 0 & $e$ \\
1 & 1 & $f$ & 0
\end{tabular}

Choix de réponses:

\begin{tabular}{|c|c|c|c|}
\hline$a=$ & 0 & $\rightarrow$ & 0.0 \\
\hline & 1 & $-\rightarrow$ & 0.1 \\
\hline$b=$ & 0 & $-->$ & 0.0 \\
\hline & 1 & $-\rightarrow$ & 0.2 \\
\hline$C=$ & 0 & $->$ & 0.0 \\
\hline & 1 & $-\rightarrow$ & 0.2 \\
\hline$d=$ & 0 & $-\rightarrow$ & 0.1 \\
\hline & 1 & $-\rightarrow$ & 0.0 \\
\hline$e=$ & 0 & $->$ & 0.2 \\
\hline & 1 & $\rightarrow$ & 0.0 \\
\hline$f=$ & 0 & $\rightarrow$ & 0.2 \\
\hline & 1 & $-\rightarrow$ & 0.0 \\
\hline
\end{tabular}

Entrer au clavier la somme des nombres correspondants aux réponses obtenues. 


\section{Activité 22}

Compléter les énoncés suivants:

$\begin{array}{llllll}\text { Si } A=1 \text { alors } & 1 \cdot A & 0 & -\rightarrow & 0.0 \\ & & 1 & -> & 0.3 \\ & & & & \\ \text { Si } A=0 \text { alors } & A \cdot 1 & = & 0 & -> & 0.3 \\ & & 1 & -> & 0.0\end{array}$

Car l'élément neutre du produit logique est:

$\begin{array}{lll}0 & -> & 0.0 \\ 1 & --> & 0.4\end{array}$

Entrer au clavier la somme des nombres correspondants aux réponses obtenues. 


\section{Activité 23}

Compléter les énoncés suivants:

$\begin{array}{llllll}\text { Si } A=0 \text { alors } & 0+A & = & 0 & -\rightarrow & 0.3 \\ & & 1 & -> & 0.0 \\ & & & & \\ \text { Si } A=1 \text { alors } & A+0= & 0 & -> & 0.0 \\ & & 1 & -\rightarrow & 0.3\end{array}$

Car l'élément neutre de la somme logique est:

$\begin{array}{lll}0 & \rightarrow & 0.4 \\ 1 & \rightarrow & 0.0\end{array}$

Entrer au clavier la somme des nombres correspondants aux réponses obtenues. 


\section{Activité 24}

Compléter les énoncés suivants:

Si $A=0$ alors

$A \cdot \bar{A}=$

0

$->$

0.2

1

0.0

Si $A=1$ alors

$\bar{A} \cdot \mathrm{A}=$

$\begin{array}{lll}0 & -> & 0.2 \\ 1 & -\rightarrow & 0.0\end{array}$

En effet, le complémentaire de A est:

$\begin{array}{lll}0 & --> & 0.0 \\ 1 & --> & 0.0 \\ \bar{A} & --> & 0.3\end{array}$

et A multiplié par son complémentaire donne toujours:

$\begin{array}{lll}0 & -> & 0.3 \\ 1 & --> & 0.0\end{array}$

Entrer au clavier la somme des nombres correspondants aux réponses obtenues. 
Activité 25

Complétèr les énoncés suivants:

$\begin{array}{lllll}\text { Si } A=0 \text { alors } & A+\bar{A}= & 0 & -\rightarrow & 0.0 \\ & & 1 & -> & 0.2 \\ & & & & \\ \text { Si } A=1 \text { alors } & \bar{A}+A= & 0 & -\rightarrow & 0.0 \\ & & 1 & -> & 0.2\end{array}$

En effet, le complémentaire de A est:

\begin{tabular}{llll}
0 & $->$ & 0.0 \\
1 & & $->$ & 0.0 \\
\hline A & $-\rightarrow$ & 0.3
\end{tabular}

et $\mathrm{A}$ additionné à son complémentaire donne toujours:

$\begin{array}{lll}0 & -> & 0.0 \\ 1 & --> & 0.3\end{array}$

Entrer au clavier la somme des nombres correspondants aux réponses obtenues. 
Activité 26

Compléter les énoncés suivants:

Si $\quad A=0$

$B=0$

$C=1$

alors
$A(B+C)$

$=$

0
1

0.3

0.0

Si $\quad A=0$

$B=1$

$C=1$

alors

$A B+A C=$

$=0$

0
1

$-\rightarrow$

0.3

$\rightarrow \quad 0.0$

Si $\quad A=0$

$B=1$

$\mathrm{C}=1$

alors

$A(B+C)=$

0

$-->$
$-->$

0.4

0.0

Entrer au clavier la somme des nombres correspondants aux réponses obtenues. 
Activité 27

Compléter les énoncés suivants:

Si $\begin{aligned} A & =0 \\ B & =0 \\ C & =1\end{aligned}$

alors

$A+(B C)=$

0
1

$\begin{array}{ll}-> & 0.3 \\ --> & 0.0\end{array}$

Si $\quad A=0$

$B=1$

C $=1$

alors $(A+B)(A+C)=$

$\begin{array}{lll}0 & -> & 0.0\end{array}$

$\begin{array}{lll}0 & -> & 0.3\end{array}$

Si $\quad A=0$

$B=1$

C $=1$

alors

$A+(B C)=$

0

$\rightarrow \quad 0.0$

$1 \quad->\quad 0.4$

Entrer au clavier la somme des nombres correspondants aux réponses obtenues. 


\section{Activité 28}

Compléter les énoncés suivants:

$$
\text { Si } \begin{aligned}
A & =0 \\
B & =1
\end{aligned}
$$

alors

$\begin{array}{llll}\overline{\mathrm{AB}}= & 0 & --> & 0.0 \\ & 1 & --> & 0.25 \\ \overline{\mathrm{A}}+\overline{\mathrm{B}}= & 0 & --> & 0.0 \\ & 1 & --> & 0.25\end{array}$

Si $\quad A=1$

$B=1$

alors

$\begin{array}{llll}\overline{\mathrm{AB}}= & 0 & --> & 0.25 \\ & 1 & --> & 0.0 \\ \overline{\mathrm{A}}+\overline{\mathrm{B}}= & 0 & -> & 0.25 \\ & 1 & -> & 0.0\end{array}$

Entrer au clavier la somme des nombres correspondants aux réponses obtenues. 


\section{Activité 29}

Compléter les énoncés suivants:

$$
\text { Si } \begin{aligned}
A & =0 \\
B & =1
\end{aligned}
$$

alors

$\begin{array}{llll}\bar{A}+\bar{B}= & 0 & -\rightarrow & 0.25 \\ & 1 & -> & 0.0 \\ \overline{A B}=0 & -> & 0.25 \\ & 1 & -> & 0.0\end{array}$

Si $\quad A=0$

$B=0$

alors

$\bar{A}+\bar{B}=\quad \begin{array}{lll}0 & --> & 0.0 \\ 1 & --> & 0.25\end{array}$

et

$\overline{\mathrm{AB}}=$

0

$\begin{array}{ll}-> & 0.0\end{array}$

1

$\begin{array}{ll}-> & 0.25\end{array}$

Entrer au clavier la somme des nombres correspondants aux réponses obtenues. 


\section{Activité 30}

De façon générale, l'algèbre de Boole est une algèbre binaire en ce sens que les variables ne peuvent prendre que deux valeurs; soit 0 ou 1.

Les opérations possibles sur les variables de Boole sont:

La somme logique:

\begin{tabular}{c|c|c}
$A$ & $B$ & $A+B$ \\
\hline 0 & 0 & 0 \\
0 & 1 & 1 \\
1 & 0 & 1 \\
1 & 1 & 1
\end{tabular}

Le produit logique:

\begin{tabular}{c|c|c}
$A$ & $B$ & $A B$ \\
\hline 0 & 0 & 0 \\
0 & 1 & 0 \\
1 & 0 & 0 \\
1 & 1 & 1
\end{tabular}

La complémentation:

\begin{tabular}{c|c}
$A$ & $\bar{A}$ \\
\hline 0 & 1 \\
1 & 0
\end{tabular}

Connaissant ces postulats de base et un certain nombre de théorèmes, on peut simplifier n'importe quelle expression booléenne 
Activité 30 (suite)

Choisir la bonne réponse aux questions suivantes:
a) $0 \cdot 1=$
0
1
$-->$
0.1
$\begin{array}{lll}1 & -> & 0.0\end{array}$
b) $1+0=$
$\begin{array}{ll}0 & --> \\ 1 & ->\end{array}$
0.0
c) $0+0$
$=$
0
$-->$
0.1
1
0.0
d) $\overline{0}$
$=$
0
$->$
$->$
0.0
1
0.1
e) $1 \cdot 1$
$=$
0
1
$->$
0.0

1
0.1
f) $\overline{1}$

$=$
0
$->$
0.1
1
$->$
0.0
g) $0+1$ =
0
1
$->$
0.0
$->$
0.1
h) $1+1=$
$\begin{array}{ll}0 & --> \\ 1 & ->\end{array}$
0.0

1
0.1
i) $0 \cdot 0$
$=$
0
1
$-->$
$-->$
0.1
0.0
j) $1 \cdot 0$
$\begin{array}{ll}= & 0 \\ 1\end{array}$
$-->$
$-->$
0.1
0.0

Entrer au clavier la somme des nombres correspondants aux réponses obtenues. 


\section{Activité 31}

L'algèbre de Boole concerne les relations entre les parties ou sous-ensembles d'un ensemble (ou référentiel). Dans les applications, on n'envisage que des ensembles finis.

II faut, avant tout, définir un référentiel $\mathrm{R}$ qui est l'ensemble de tous les ensembles qu'on considère dans un problème. Il est d'usage de désigner le référentiel par 1 et l'ensemble vide $\phi$ par 0 .

On note un ensemble quelconque par son nom $A, B, C, \ldots$ et on peut le représenter graphiquement par un diagramme de Venn.

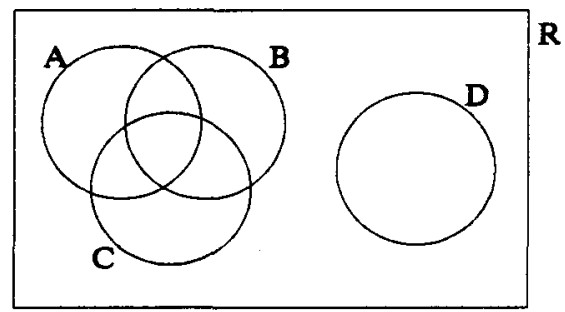

Les opérations à considérer sont:

-la réunion de deux ensembles: A U B

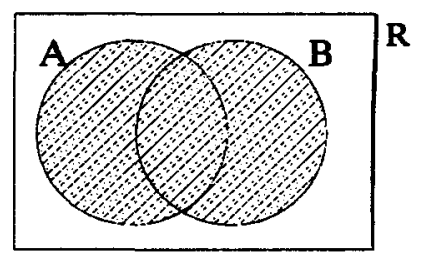

-l'intersection de deux ensembles: $A \cap B$

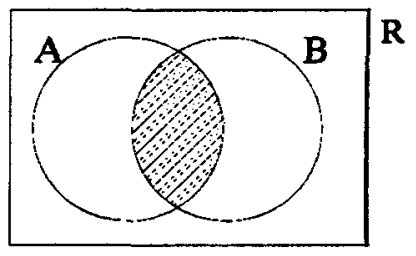




\section{Activité 31 (suite)}

-le complément d'un ensemble: $\overline{\mathrm{A}}$

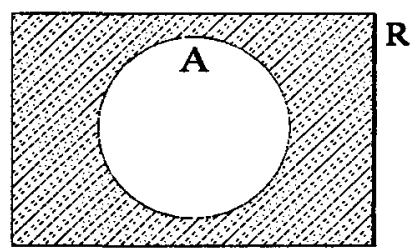

Parmi les diagrammes de Venn suivants:

(1)

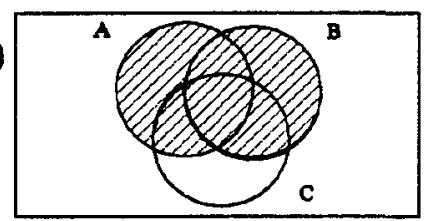

(3)

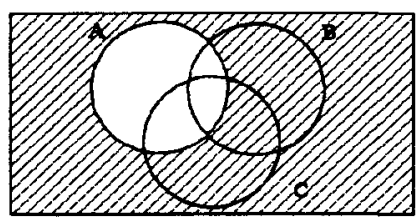

(5)

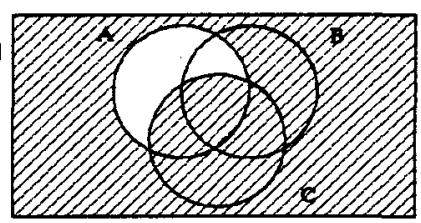

(7)

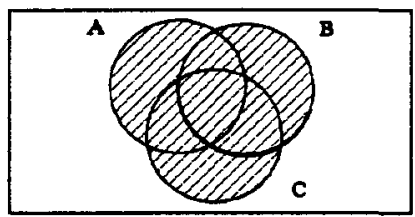

(9)

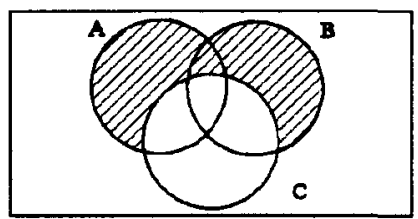

(2)

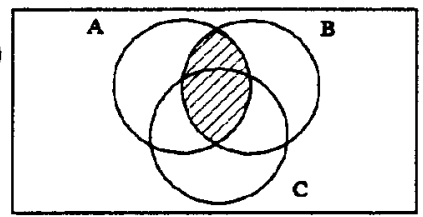

(4)

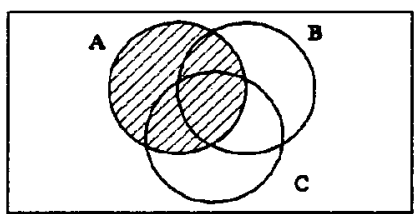

(6)

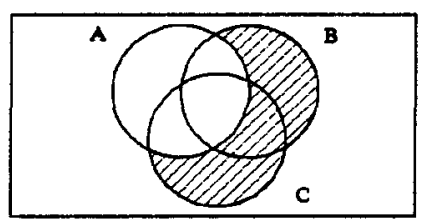

(8)

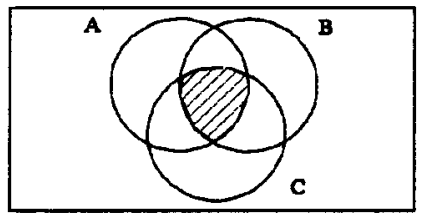

(10)

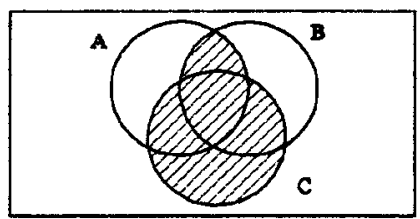


Activité 31 (suite)

choisir la bonne représentation pour les expressions qui suivent:

a) $A \cup B$ :

(1)

(2)

(3)

(4)

(5)

(6)

(7)

(8)

(9)

(10)

b) $A \cap B:$

c) $\bar{A}$ :
(1)

(2)

(3)

(4)

(5)

(6)

(7)

(8)

(9)

(10)

(1)

(2)

(3)

(4)

(5)

(6)

(7)

(8)

(9)

(10)

$\begin{array}{ll}--> & 0.1 \\ --> & 0.0 \\ --> & 0.0 \\ --> & 0.0 \\ -> & 0.0 \\ -> & 0.0 \\ --> & 0.0 \\ -> & 0.0 \\ --> & 0.0 \\ -> & 0.0\end{array}$

$\begin{array}{ll}-> & 0.0\end{array}$

$\rightarrow \quad 0.1$

$\begin{array}{ll}-> & 0.0\end{array}$

$\begin{array}{ll}-> & 0.0\end{array}$

$\begin{array}{ll}-\rightarrow & 0.0\end{array}$

$\begin{array}{ll}-> & 0.0\end{array}$

$\begin{array}{ll}-> & 0.0\end{array}$

$\begin{array}{ll}-> & 0.0\end{array}$

$\begin{array}{ll}-> & 0.0\end{array}$

$\begin{array}{ll}-> & 0.0\end{array}$

$\begin{array}{ll}-> & 0.0\end{array}$

$\begin{array}{ll}-> & 0.0\end{array}$

$\begin{array}{ll}-> & 0.1\end{array}$

$\begin{array}{ll}-> & 0.0\end{array}$

$\begin{array}{ll}-> & 0.0\end{array}$

$\begin{array}{ll}-> & 0.0\end{array}$

$\begin{array}{ll}-> & 0.0\end{array}$

$\begin{array}{ll}-> & 0.0\end{array}$

$\begin{array}{ll}-> & 0.0\end{array}$

$\begin{array}{ll}-> & 0.0\end{array}$ 
Activité 31 (suite)

d) $\bar{A} \cup \mathrm{C}:$

e) $A \cap B \cup C:$
(1)

(10)

$\begin{array}{ll}-> & 0.0 \\ -> & 0.0 \\ -> & 0.0 \\ -> & 0.0 \\ -> & 0.1 \\ -> & 0.0 \\ -> & 0.0 \\ -> & 0.0 \\ -> & 0.0 \\ -> & 0.0\end{array}$

$\begin{array}{ll}-> & 0.0\end{array}$

$\rightarrow \quad 0.0$

$\begin{array}{ll}-> & 0.0\end{array}$

$\begin{array}{ll}-> & 0.0\end{array}$

$\begin{array}{ll}-> & 0.0\end{array}$

$\begin{array}{ll}-> & 0.0\end{array}$

$\begin{array}{ll}-> & 0.0\end{array}$

$\begin{array}{ll}-> & 0.0\end{array}$

$\begin{array}{ll}-> & 0.0\end{array}$

$\begin{array}{ll}-> & 0.1\end{array}$

L'algèbre de Boole est basée sur un certain nombre de postulats que voici:

\section{Postulat du ET:}

Soit $A$ et $B$, deux ensembles quelconques, alors $A \cap B$ représente un nouvel ensemble qui contient les éléments qui sont à la fois dans l'ensemble $A$ et l'ensemble B.

Si les ensembles $A$ et $B$ ne peuvent être que l'ensemble vide $\phi$ ou le référentiel $R$, alors on peut affirmer les postulats suivants:

$$
\begin{aligned}
& \phi \cap \phi=\phi \\
& \phi \cap R=\phi \\
& R \cap \phi=\phi \\
& R \cap R=R
\end{aligned}
$$

qu'on peut résumer de la façon suivante en se souvenant que 1 représente le référentiel $R$ et que 0 représente l'ensemble vide $\phi$ :

$$
\begin{aligned}
& 0 \cdot 0=0 \\
& 0 \cdot 1=0 \\
& 1 \cdot 0=0 \\
& 1 \cdot 1=1
\end{aligned}
$$


Activité 31 (suite)

Postulats du OU:

Soit $A$ et $B$, deux ensembles quelconques, alors $A \cup B$ représente un nouvel ensemble qui contient les éléments qui sont dans $A$ ou encore dans $B$.

Si les ensembles $A$ et $B$ ne peuvent être que l'ensemble vide $\phi$ ou le référentiel $R$, alors on peut affirmer les postulats suivants:

$\phi \cup \phi=\phi$

$\phi \cup R=R$

$R \cup \phi=R$

$R \cup R=R$

qu'on peut résumer de la façon suivante en se souvenant que 1 représente le référentiel $R$ et que 0 représente l'ensemble vide $\phi: \quad 0+0=0$

$0+1=1$

$1+0=1$

$1+1=1$

Postulats du NON:

Soit $A$ un ensemble quelconque, alors on désigne $\bar{A}$ le complément de l'ensemble $A$, on peut donc dire que $\bar{A}$ est la négation de $A$.

Si l'ensemble A ne peut être que l'ensemble vide $\phi$ ou le référentiel $R$, alors on peut affirmer les postulats suivants: $\quad \bar{\phi}=R$

$$
\overline{\mathrm{R}}=\phi
$$

ou encore:

$$
\begin{aligned}
& \overline{0}=1 \\
& \overline{1}=0
\end{aligned}
$$


Activité 31 (suite)

En se souvenant que 1 représente l'ensemble référentiel $R, 0$ l'ensemble vide $f$, • l'intersection $(E T),+$ la réunion $(\mathrm{OU})$ et le complément (NON), choisir la bonne réponse aux questions suivantes:
a) $0 \cdot 1=$
$0 \quad->$
0.1
$1 \quad-\rightarrow \quad 0.0$
b) $1+0=$
$\begin{array}{lll}0 & --> & 0.0 \\ 1 & --> & 0.1\end{array}$
c) $\overline{0}=$
$0 \quad->\quad 0.0$
$1 \quad->\quad 0.1$
d) $1 \cdot 1=$

$\begin{array}{lll}0 & -> & 0.0 \\ 1 & --> & 0.1\end{array}$
e) $1+1=$
$0 \quad->$
0.0
$1 \quad \rightarrow \quad 0.1$

Entrer au clavier la somme des nombres correspondants aux réponses obtenues. 


\section{Activité 32}

L'algèbre de Boole est une algèbre binaire, ce qui veut dire que les différentes variables ne peuvent prendre que les deux valeurs numériques 0 ou 1 et aucune autre.

La propriété caractéristique des nombres binaires est d'être égaux à leur carré, ainsi on a:

$$
0^{2}=0 \cdot 0=0
$$

et

$$
1^{2}=1 \cdot 1=1
$$

\section{Postulats du ET:}

Soit $A$ et $B$, deux nombres binaires, alors $A \cdot B$ est le produit logique de $A$ par $B$ et on peut affirmer les postulats suivants:

$$
\begin{aligned}
& 0 \cdot 0=0 \\
& 0 \cdot 1=0 \\
& 1 \cdot 0=0 \\
& 1 \cdot 1=1
\end{aligned}
$$

En admettant de la même façon pour la somme logique que la somme d'un nombre booléen par lui-même est égale à ce nombre, on obtient:

$$
\text { et } \quad \begin{aligned}
& 0+0=0 \\
& 1+1=1
\end{aligned}
$$

\section{Postulats du OU:}

Soit $A$ et $B$, deux nombres binaires, alors $A+B$ est la somme logique de $A$ et $B$, on peut alors affirmer les postulats suivants:

$$
\begin{aligned}
& 0+0=0 \\
& 0+1=1 \\
& 1+0=1 \\
& 1+1=1
\end{aligned}
$$

\section{Postulats du NON:}

Soit $A$, un nombre binaire quelconque, alors $\bar{A}$ est appelé le complément de $A$ ou la négation de $A$ et on peut affirmer les postulats suivants: $\quad \overline{0}=1$

$$
\overline{1}=0
$$


Activité 32 (suite)

En se souvenant que l'algèbre de Boole est une algèbre binaire, choisir la bonne réponse aux questions suivantes:
a) $0 \cdot 1=$
0
$\begin{array}{ll}-> & 0.2 \\ -> & 0.0\end{array}$
b) $1+0=$

$\begin{array}{lll}0 & -> & 0.0 \\ 1 & -> & 0.2\end{array}$
c) $\overline{0}=$

$\begin{array}{lll}0 & -> & 0.0 \\ 1 & -> & 0.2\end{array}$
d) $1 \cdot 1=$

$\begin{array}{lll}0 & -> & 0.0 \\ 1 & -> & 0.2\end{array}$
e) $1+1=$

$\begin{array}{lll}0 & -> & 0.0 \\ 1 & -\rightarrow & 0.2\end{array}$

Entrer au clavier la somme des nombres correspondants aux réponses obtenues. 
Activité 33

L'algèbre de Boole est une algèbre logique dans laquelle on utilise des valeurs numériques à des fins logiques.

Soit $A$ et $B$, deux propositions logiques comme

$$
\begin{aligned}
& A=\text { "Pierre aime jouer au baseball" } \\
& B=\text { "Paul aime jouer au hockey" }
\end{aligned}
$$

On peut assigner à ces propositions des valeurs logiques en désignant chacune d'elles comme étant vraie ou fausse. Afin de faciliter la notation, on donne à la valeur logique "vraie" la valeur numérique 1 et à la valeur logique "fausse" la valeur numérique 0.

Ainsi, supposons que Pierre n'aime pas jouer au baseball et que Paul aime jouer au hockey, alors on peut écrire

$$
\begin{aligned}
& A=0 \\
& B=1
\end{aligned}
$$


Activité 33 (suite)

Se souvenant qu'on donne la valeur 0 à une proposition fausse et 1 à une proposition vraie, supposons les propositions suivantes:

$$
\begin{aligned}
& \text { A = "Tu aimes regarder le hockey à la télévision" } \\
& B=\text { "Tu aimes aller voir une partie de baseball" } \\
& C=\text { "Tu aime jouer à la balle molle" }
\end{aligned}
$$

Si ces trois affirmations sont vraies, alors choisir la bonne valeur pour les propositions suivantes:

$$
\begin{array}{lll} 
& D= \\
D= & \text { "Tu n'aimes pas jouer à la balle molle" } \\
0 & -\rightarrow & 0.1 \\
1 & -\rightarrow & 0.0
\end{array}
$$

$E=\quad$ "Tu aimes regarder le hockey à la télévision et aller voir une partie de baseball"

$E=\begin{array}{lll}0 & -> & 0.0 \\ 1 & -> & 0.1\end{array}$

$F=\quad$ "Tu aimes regarder le hockey à la télévision et tu n'aimes pas jouer à la balle molle"

$$
F=\begin{array}{lll}
0 & -> & 0.1 \\
1 & -> & 0.0
\end{array}
$$

$\mathrm{G}=\quad$ "Tu n'aimes pas jouer à la balle molle ou tu aimes aller voir une partie de baseball

$$
G=\begin{array}{lll}
0 & -> & 0.1 \\
1 & --> & 0.0
\end{array}
$$


Activité 33 (suite)

\section{Postulat duET:}

Soit A et B, deux propositions comme:

$$
\begin{aligned}
& A=\text { "Pierre aime jouer au baseball" } \\
& B=\text { "Paul aime jouer au hockey" }
\end{aligned}
$$

alors on note A.B la nouvelle proposition:

"Pierre aime jouer au baseball et Paul aime jouer au hockey"

La valeur de la nouvelle proposition dépend des valeurs des propositions $A$ et $B$. Ainsi on peut affirmer les résultats suivants:

Si $A$ est vraie et $B$ est vraie, alors $A \cdot B$ est vraie

Si $A$ est vraie et $B$ est fausse, alors $A \cdot B$ est fausse

Si $A$ est fausse et $B$ est vraie, alors $A \cdot B$ est fausse

Si A est fausse et $B$ est fausse, alors $A \cdot B$ est fausse

qu'on peut résumer de la façon suivante:

$$
\begin{aligned}
& 1 \cdot 1=1 \\
& 1 \cdot 0=0 \\
& 0 \cdot 1=0 \\
& 0 \cdot 0=0
\end{aligned}
$$


Activité 33 (suite)

\section{Postulat du OU:}

Soit $A$ et $B$, deux propositions comme:

$$
\begin{aligned}
& \text { A = "Pierre aime jouer au baseball" } \\
& \text { B = "Paul aime jouer au hockey" }
\end{aligned}
$$

alors on note $A+B$ la nouvelle proposition:

"Pierre aime jouer au baseball ou Paul aime jouer au hockey"

La valeur de la nouvelle proposition dépend des valeurs des propositions $A$ et $B$. Ainsi on peut affirmer les résultats suivants:

Si $A$ est vraie et $B$ est vraie, alors $A+B$ est vraie

Si $A$ est vraie et $B$ est fausse, alors $A+B$ est vraie

Si $A$ est fausse et $B$ est vraie, alors $A+B$ est vraie

Si $A$ est fausse et $B$ est fausse, alors $A+B$ est fausse

qu'on peut résumer de la façon suivante:

$$
\begin{aligned}
& 1+1=1 \\
& 1+0=1 \\
& 0+1=1 \\
& 0+0=0
\end{aligned}
$$


Activité 33 (suite)

\section{Postulat du NON:}

Soit une proposition comme:

$$
A=\text { "Tu aimes faire du ski" }
$$

alors on note $\overline{\mathrm{A}}$ la proposition contraire qui est:

$$
\bar{A}=\text { "Tu n'aimes pas faire du ski" }
$$

La valeur de la proposition $\overline{\mathrm{A}}$ dépend donc de celle de la proposition $\mathrm{A}$ de la manière suivante:

Si $A$ est vraie alors $\bar{A}$ est fausse

Si $A$ est fausse alors $\bar{A}$ est vraie

qu'on peut résumer de la façon suivante:

$$
\begin{aligned}
& \overline{1}=0 \\
& \overline{0}=1
\end{aligned}
$$




\section{Activité 33 (suite)}

En se souvenant que dans l'algèbre de Boole les propositions peuvent prendre seulement deux valeurs ( 0 ou 1 ) et que les opérations - représente le ET logique, + représente le OU logique et représente la négation de la proposition, choisir la bonne réponse pour les assertions suivantes:
a) $0 \cdot 1=$
$0 \quad \rightarrow$
0.1
$1 \quad-\rightarrow \quad 0.0$
b) $1+0=$

$\begin{array}{lll}0 & -> & 0.0 \\ 1 & -\rightarrow & 0.1\end{array}$
c) $\overline{0}=$
$0 \quad-\rightarrow \quad 0.0$
$1 \quad-\rightarrow \quad 0.1$
d) $1+1=$
$0 \quad->\quad 0.0$
$1 \quad->\quad 0.1$
e) $1 \cdot 1=$

$\begin{array}{lll}0 & -> & 0.0 \\ 1 & --> & 0.1\end{array}$
f) $0+0=$

$\begin{array}{lll}0 & -> & 0.0 \\ 1 & -\rightarrow & 0.1\end{array}$

Entrer au clavier la somme des nombres correspondants aux réponses obtenues. 


\section{PARTIE 5}

\section{SIMULATIONS DE PROLIN}




\section{PARTIE 5}

\section{SIMULATIONS DE PROLIN}

Suivent les sommaires de 11 résultats de simulations du programme PROLIN. Ils sont placés dans des tableaux afin de permettre une meilleure lisibilité. Vous trouverez la sortie d'imprimante obtenue lors de la simulation comme telle du cas numéro 11 à la partie 6 de ce même document. 


\section{SOMMAIRE DES RESULTATS DE 11 SIMULATIONS DE PROLIN}

\section{Cas 1:}

Profil simulé: Etudiant moyen qui obtient des résultats parfaits partout.

Pourcentage visuel: $\quad 0.0$

Pourcentage auditif: $\quad 0.8$

Pourcentage kinesthésique: $\quad 0.2$

Vitesse d'apprentissage: $\quad 0.6$

\begin{tabular}{|c|c|c|c|c|}
\hline & CONTEXTE & ACTIVITE & RESULTAT & PROGRAMME \\
\hline 1 & $\begin{array}{l}\text { 5: L'apprenant est } \\
\text { auditif et moyen }\end{array}$ & $\begin{array}{l}\text { 30: Compléter des } \\
\text { tables de vérité sur les } \\
\text { postulats } \\
\text { note: } 1.0\end{array}$ & $\begin{array}{l}\text { 1: Utilisation des } \\
\text { théorèmes de } \\
\text { l'élément neutre }\end{array}$ & $\begin{array}{l}\text { 2: Introduction à } \\
\text { l'algèbre de Boole }\end{array}$ \\
\hline 2 & $\begin{array}{l}\text { 7: L'apprenant est } \\
\text { kinesthésique et lent }\end{array}$ & $\begin{array}{l}\text { 26: Résoudre } \\
\text { (distributivité du } \\
\text { produit sur la somme } \\
\text { logique) } \\
\text { note: } 1.0\end{array}$ & $\begin{array}{l}\text { 3: Utilisation des } \\
\text { théorèmes de la } \\
\text { distributivité }\end{array}$ & $\begin{array}{l}\text { 13: Théorème de la } \\
\text { distributivité de la } \\
\text { somme sur le produit } \\
\text { logique }\end{array}$ \\
\hline 3 & $\begin{array}{l}\text { 6: L'apprenant est } \\
\text { auditif et rapide }\end{array}$ & $\begin{array}{c}\text { 27: Résoudre } \\
\text { (distributivité de la } \\
\text { somme sur le produit } \\
\text { logique) } \\
\text { note: } 1.0\end{array}$ & $\begin{array}{c}\text { 7: Simplification de } \\
\text { l'expression booléenne } \\
\text { prédéfinie }\end{array}$ & $\begin{array}{c}\text { 6: Les théorèmes de } \\
\text { niveau } 2\end{array}$ \\
\hline 4 & $\begin{array}{l}\text { 9: L'apprenant est } \\
\text { kinesthésique et } \\
\text { rapide }\end{array}$ & $\begin{array}{c}\text { 12: Simplifier } \\
\text { (distributivité et De } \\
\text { Morgan) } \\
\text { note: } 1.0\end{array}$ & $\begin{array}{c}\text { 4: Utilisation des } \\
\text { théorèmes des lois de } \\
\text { De Morgan }\end{array}$ & $\begin{array}{l}\text { 13: Théorème de la } \\
\text { distributivité de la } \\
\text { somme sur le produit } \\
\text { logique }\end{array}$ \\
\hline 5 & $\begin{array}{l}\text { 6: L'apprenant est } \\
\text { auditif et rapide }\end{array}$ & $\begin{array}{l}\text { 19: Table de vérité } \\
\text { (complémentation) } \\
\text { note: } 1.0\end{array}$ & $\begin{array}{l}\text { 2: Utilisation des } \\
\text { théorèmes de la } \\
\text { complémentation }\end{array}$ & $\begin{array}{l}\text { 12: Théorème de la } \\
\text { distributivité du produit } \\
\text { sur la somme logique }\end{array}$ \\
\hline 6 & $\begin{array}{l}\text { 9: L'apprenant est } \\
\text { kinesthésique et } \\
\text { rapide }\end{array}$ & $\begin{array}{l}\text { 8:Simplifier (élément } \\
\text { neutre et distributivité) } \\
\text { note: } 1.0\end{array}$ & $\begin{array}{l}\text { 5: Simplification } \\
\text { d'expressions } \\
\text { booléennes à } 2 \\
\text { variables }\end{array}$ & $\begin{array}{l}\text { 8: Théorème de } \\
\text { l'élément neutre du } \\
\text { produit logique }\end{array}$ \\
\hline 7 & $\begin{array}{l}\text { 6: L'apprenant est } \\
\text { auditif et rapide }\end{array}$ & $\begin{array}{c}\text { 10: Simplifier } \\
\text { (distributivité et De } \\
\text { Morgan) } \\
\text { note: } 1.0\end{array}$ & $\begin{array}{l}\text { 5: Simplification } \\
\text { d'expressions } \\
\text { booléennes à } 2 \\
\text { variables }\end{array}$ & $\begin{array}{l}\text { 10: Théorème de la } \\
\text { complémentation du } \\
\text { produit logique }\end{array}$ \\
\hline
\end{tabular}




\begin{tabular}{|c|c|c|c|c|}
\hline 8 & $\begin{array}{c}\text { 9: L'apprenant est } \\
\text { kinesthésique et } \\
\text { rapide }\end{array}$ & $\begin{array}{l}\text { 9: Simplifier (élément } \\
\text { neutre et De Morgan) } \\
\text { note: } 1.0\end{array}$ & $\begin{array}{l}\text { 5: Simplification } \\
\text { d'expressions } \\
\text { booléennes à } 2 \\
\text { variables }\end{array}$ & $\begin{array}{c}\text { 14: La première loi de } \\
\text { De Morgan }\end{array}$ \\
\hline 9 & $\begin{array}{l}\text { 6: L'apprenant est } \\
\text { auditif et rapide }\end{array}$ & $\begin{array}{l}\text { 11: Simplifier } \\
\text { (complémentation et } \\
\text { De Morgan) } \\
\text { note: } 1.0\end{array}$ & $\begin{array}{l}\text { 5: Simplification } \\
\text { d'expressions } \\
\text { booléennes à } 2 \\
\text { variables }\end{array}$ & $\begin{array}{c}\text { 15: La deuxième loi de } \\
\text { De Morgan }\end{array}$ \\
\hline 10 & $\begin{array}{c}\text { 9: L'apprenant est } \\
\text { kinesthésique et } \\
\text { rapide }\end{array}$ & $\begin{array}{l}\text { 7: Simplifier (élément } \\
\text { neutre et } \\
\text { complémentation) } \\
\text { note: } 1.0\end{array}$ & $\begin{array}{l}\text { 5: Simplification } \\
\text { d'expressions } \\
\text { booléennes à } 2 \\
\text { variables }\end{array}$ & $\begin{array}{l}\text { 9: Théorème de } \\
\text { l'élément neutre de la } \\
\text { somme logique }\end{array}$ \\
\hline 11 & $\begin{array}{l}\text { 6: L'apprenant est } \\
\text { auditif et rapide }\end{array}$ & $\begin{array}{l}\text { 21: Tables de vérité } \\
\text { (De Morgan) } \\
\text { note: } 1.0\end{array}$ & $\begin{array}{l}\text { 2: Utilisation des } \\
\text { théorèmes de la } \\
\text { complémentation }\end{array}$ & $\begin{array}{l}\text { 12: Théorème de la } \\
\text { distributivité du produit } \\
\text { sur la somme logique }\end{array}$ \\
\hline 12 & $\begin{array}{c}\text { 9: L'apprenant est } \\
\text { kinesthésique et } \\
\text { rapide }\end{array}$ & $\begin{array}{l}\text { 18: Tables de vérité } \\
\text { (élément neutre) } \\
\text { note: } 1.0\end{array}$ & $\begin{array}{l}\text { 2: Utilisation des } \\
\text { théorèmes de la } \\
\text { complémentation }\end{array}$ & $\begin{array}{l}\text { 11: Théorème de la } \\
\text { complémentation de la } \\
\text { somme logique }\end{array}$ \\
\hline 13 & $\begin{array}{l}\text { 6: L'apprenant est } \\
\text { auditif et rapide }\end{array}$ & $\begin{array}{l}\text { 3: Simplifier (élément } \\
\text { neutre, } \\
\text { complémentation et } \\
\text { distributivité) } \\
\text { note: } 1.0\end{array}$ & $\begin{array}{l}\text { 5: Simplification } \\
\text { d'expressions } \\
\text { booléennes à } 2 \\
\text { variables }\end{array}$ & $\begin{array}{l}\text { 1: Simplification de } \\
\text { l'expression booléenne } \\
\text { donnée }\end{array}$ \\
\hline 14 & $\begin{array}{l}\text { 3: L'apprenant est } \\
\text { visuel et rapide }\end{array}$ & $\begin{array}{l}\text { 1: Simplifier } \\
\text { l'expression donnée } \\
\text { note: } 1.0\end{array}$ & $\begin{array}{l}\text { 2: Utilisation des } \\
\text { théorèmes de la } \\
\text { complémentation }\end{array}$ & \\
\hline
\end{tabular}

Réussite de l'objectif final.

Remarquons que la seule différence ici avec le premier cas (cas 1) présenté au chapitre 8 du mémoire se situe au niveau de la vitesse d'apprentissage de l'apprenant. Cette dernière a été définie comme étant moyenne. PROLIN offre donc une progression du même type que pour le cas 1 du mémoire mais utilise plus d'étapes. En fait, l'apprenant aura deux fois plus d'activités (14 au lieu de 7 dans le cas 1 du mémoire) à faire ici avant d'atteindre l'objectif final. PROLIN vérifie l'acquisition des connaissances en demandant à l'usager de compléter des tables de vérités (aux étapes 5,11 et 18) et offre plus d'activités de simplification qu'à un apprenant rapide comme dans le cas 1 du mémoire. 


\section{Cas 2:}

Profil simulé: Etudiant rapide mais instable dans ses résultats.

Pourcentage visuel:

0.8

Pourcentage auditif:

0.2

Pourcentage kinesthésique:

0.0

Vitesse d'apprentissage:

0.8

\begin{tabular}{|c|c|c|c|c|}
\hline & CONTEXTE & ACTIVITE & RESULTAT & PROGRAMME \\
\hline 1 & $\begin{array}{l}\text { 3: L'apprenant est } \\
\text { visuel et rapide }\end{array}$ & $\begin{array}{c}\text { 30: Compléter des } \\
\text { tables de vérité sur les } \\
\text { postulats } \\
\text { note: } 1.0 \\
\end{array}$ & $\begin{array}{l}\text { 1: Utilisation des } \\
\text { théorèmes de } \\
\text { l'élément neutre }\end{array}$ & $\begin{array}{l}\text { 2: Introduction à } \\
\text { l'algèbre de Boole }\end{array}$ \\
\hline 2 & $\begin{array}{l}\text { 7: L'apprenant est } \\
\text { kinesthésique et lent }\end{array}$ & $\begin{array}{c}\text { 26: Résoudre } \\
\text { (distributivité du } \\
\text { produit sur la somme } \\
\text { logique) } \\
\text { note: } 0.5\end{array}$ & $\begin{array}{l}\text { 3: Utilisation des } \\
\text { théorèmes de la } \\
\text { distributivité }\end{array}$ & $\begin{array}{l}\text { 2: Introduction à } \\
\text { l'algèbre de Boole }\end{array}$ \\
\hline 3 & $\begin{array}{l}\text { 1: L'apprenant est } \\
\text { visuel et lent }\end{array}$ & $\begin{array}{c}\text { 29: Résoudre } \\
\text { (deuxième loi de De } \\
\text { Morgan) } \\
\text { note: } 0.0\end{array}$ & $\begin{array}{l}\text { 2: Utilisation des } \\
\text { théorèmes de la } \\
\text { complémentation }\end{array}$ & $\begin{array}{l}\text { 2: Introduction à } \\
\text { l'algèbre de Boole }\end{array}$ \\
\hline 4 & $\begin{array}{l}\text { 1: L'apprenant est } \\
\text { visuel et lent }\end{array}$ & $\begin{array}{c}\text { 27: Résoudre } \\
\text { (distributivité de la } \\
\text { somme sur le produit } \\
\text { logique) } \\
\text { note: } 0.5\end{array}$ & $\begin{array}{l}\text { 1: Utilisation des } \\
\text { théorèmes de } \\
\text { l'élément neutre }\end{array}$ & $\begin{array}{l}\text { 13: Théorème de la } \\
\text { distributivité de la } \\
\text { somme sur le produit } \\
\text { logique }\end{array}$ \\
\hline 5 & $\begin{array}{l}\text { 3: L'apprenant est } \\
\text { visuel et rapide }\end{array}$ & $\begin{array}{l}\text { 1: Simplifier } \\
\text { l'expression donnée } \\
\text { note: } 1.0\end{array}$ & $\begin{array}{c}\text { 8: Utilisation des } \\
\text { postulats }\end{array}$ & \\
\hline
\end{tabular}

Réussite de l'objectif final.

Cet apprenant présentant beaucoup d'instabilité, PROLIN ne sait pas trop comment agir. Face à la faible performance de l'usager à l'étape 2, PROLIN lui propose d'autres activités du même type (étape 3 et 4), puis lui proposera assez 
rapidement, dès l'étape 5, l'activité finale (1: Simplifier l'expression donnée) pour lequel par hasard, la réponse donnée par l'apprenant sera la bonne.

Le comportement de PROLIN ressemble ici à celui de I'humain. En effet, face à ce type de comportement d'un étudiant, l'enseignant ne sachant trop quelle attitude adopter, choisira probablement d'aller vérifier au plus tôt l'objectif final. 


\section{Cas 3:}

Profil simulé: Etudiant moyen mais instable dans ses résultats.

Pourcentage visuel: $\quad 0.8$

Pourcentage auditif: $\quad 0.2$

Pourcentage kinesthésique: $\quad 0.0$

Vitesse d'apprentissage: $\quad 0.6$

\begin{tabular}{|c|c|c|c|c|}
\hline & CONTEXTE & ACTIVITE & RESULTAT & PROGRAMME \\
\hline 1 & $\begin{array}{l}\text { 2: L'apprenant est } \\
\text { visuel et moyen }\end{array}$ & $\begin{array}{c}\text { 30: Compléter des } \\
\text { tables de vérité sur les } \\
\text { postulats } \\
\text { note: } 1.0 \\
\end{array}$ & $\begin{array}{l}\text { 1: Utilisation des } \\
\text { théorèmes de } \\
\text { l'élément neutre }\end{array}$ & $\begin{array}{l}\text { 2: Introduction à } \\
\text { l'algèbre de Boole }\end{array}$ \\
\hline 2 & $\begin{array}{l}\text { 7: L'apprenant est } \\
\text { kinesthésique et lent }\end{array}$ & $\begin{array}{l}\text { 26: Résoudre } \\
\text { (distributivité du } \\
\text { produit sur la somme } \\
\text { logique) } \\
\text { note: } 0.5\end{array}$ & $\begin{array}{c}\text { 7: Simplification de } \\
\text { l'expression booléenne } \\
\text { prédéfinie }\end{array}$ & $\begin{array}{l}\text { 8: Théorème de } \\
\text { l'élément neutre du } \\
\text { produit logique }\end{array}$ \\
\hline 3 & $\begin{array}{l}\text { 3: L'apprenant est } \\
\text { visuel et rapide }\end{array}$ & $\begin{array}{l}\text { 18: Tables de vérité } \\
\text { (élément neutre) } \\
\text { note: } 0.0\end{array}$ & $\begin{array}{l}\text { 1: Utilisation des } \\
\text { théorèmes de } \\
\text { l'élément neutre }\end{array}$ & $\begin{array}{l}\text { 3: Simplification } \\
\text { d'expressions } \\
\text { booléennes }\end{array}$ \\
\hline 4 & $\begin{array}{l}\text { 9: L'apprenant est } \\
\text { kinesthésique et } \\
\text { rapide }\end{array}$ & $\begin{array}{l}\text { 2: Simplifier (ensemble } \\
\text { des théorèmes) } \\
\text { note: } 0.5\end{array}$ & $\begin{array}{l}\text { 3: Utilisation des } \\
\text { théorèmes de la } \\
\text { distributivité }\end{array}$ & $\begin{array}{l}\text { 3: Simplification } \\
\text { d'expressions } \\
\text { booléennes }\end{array}$ \\
\hline 5 & $\begin{array}{l}\text { 6: L'apprenant est } \\
\text { auditif et rapide }\end{array}$ & $\begin{array}{l}\text { 1: Simplifier } \\
\text { l'expression donnée } \\
\text { note: } 1.0\end{array}$ & $\begin{array}{l}\text { 8: Utilisation des } \\
\text { postulats }\end{array}$ & \\
\hline
\end{tabular}

Réussite de l'objectif final.

Cet étudiant offre des résultats instables comme le précédant sauf qu'il est défini au départ comme ayant une vitesse d'apprentissage moyenne. PROLIN s'attend à lui offrir une progression plus lente d'activités mais les réponses démontrent que l'élève a plus de facilité avec les activités difficiles (résoudre et simplifier aux étapes 2 et 4) qu'avec les plus simples (tables de vérité à l'étape 3). II 
adoptera donc la même attitude que pour le cas précédent et offrira rapidement l'activité finale dès l'étape 5. 


\section{Cas 4:}

Profil simulé: Etudiant moyen et instable.

Pourcentage visuel: $\quad 0.8$

Pourcentage auditif: $\quad 0.2$

Pourcentage kinesthésique: $\quad 0.0$

Vitesse d'apprentissage: $\quad 0.6$

\begin{tabular}{|c|c|c|c|c|}
\hline & CONTEXTE & ACTIVITE & RESULTAT & PROGRAMME \\
\hline 1 & $\begin{array}{l}\text { 2: L'apprenant est } \\
\text { visuel et moyen }\end{array}$ & $\begin{array}{c}\text { 30: Compléter des } \\
\text { tables de vérité sur les } \\
\text { postulats } \\
\text { note: } 0.0\end{array}$ & $\begin{array}{c}\text { 7: Simplification de } \\
\text { l'expression booléenne } \\
\text { prédéfinie }\end{array}$ & $\begin{array}{c}\text { aucun } \\
\text { Niveau } 2\end{array}$ \\
\hline 2 & & & $\begin{array}{l}\text { 8: Utilisation des } \\
\text { postulats }\end{array}$ & $\begin{array}{c}\text { 3: Simplification } \\
\text { d'expressions } \\
\text { booléennes }\end{array}$ \\
\hline 3 & $\begin{array}{l}\text { 2: L'apprenant est } \\
\text { visuel et moyen }\end{array}$ & $\begin{array}{l}\text { 1: Simplifier } \\
\text { l'expression donnée } \\
\text { note: } 0.5\end{array}$ & \begin{tabular}{|c|} 
7: Simplification de \\
l'expression booléenne \\
prédéfinie
\end{tabular} & $\begin{array}{l}\text { 3: Simplification } \\
\text { d'expressions } \\
\text { booléennes }\end{array}$ \\
\hline 4 & $\begin{array}{l}\text { 3: L'apprenant est } \\
\text { visuel et rapide }\end{array}$ & $\begin{array}{c}\text { 10: Simplifier } \\
\text { (distributivité et De } \\
\text { Morgan) } \\
\text { note: } 1.0\end{array}$ & $\begin{array}{l}\text { 2: Utilisation des } \\
\text { théorèmes de la } \\
\text { complémentation }\end{array}$ & $\begin{array}{c}\text { 6: Les théorèmes de } \\
\text { niveau } 2\end{array}$ \\
\hline 5 & $\begin{array}{l}\text { 9: L'apprenant est } \\
\text { kinesthésique et } \\
\text { rapide }\end{array}$ & $\begin{array}{c}\text { 3: Simplifier (élément } \\
\text { neutre, } \\
\text { complémentation et } \\
\text { distributivité) } \\
\text { note: } 0.5\end{array}$ & $\begin{array}{l}\text { 3: Utilisation des } \\
\text { théorèmes de la } \\
\text { distributivité }\end{array}$ & $\begin{array}{l}\text { 3: Simplification } \\
\text { d'expressions } \\
\text { booléennes }\end{array}$ \\
\hline 6 & $\begin{array}{l}\text { 6: L'apprenant est } \\
\text { auditif et rapide }\end{array}$ & $\begin{array}{c}\text { 13: Simplifier (élément } \\
\text { neutre) } \\
\text { note: } 0.0\end{array}$ & $\begin{array}{c}\text { 5: Simplification } \\
\text { d'expressions } \\
\text { booléennes à } 2 \\
\text { variables } \\
\end{array}$ & $\begin{array}{l}\text { 13: Théorème de la } \\
\text { distributivité de la } \\
\text { somme sur le produit } \\
\text { logique }\end{array}$ \\
\hline 7 & $\begin{array}{l}\text { 3: L'apprenant est } \\
\text { visuel et rapide }\end{array}$ & $\begin{array}{l}\text { 8: Simplifier (élément } \\
\text { neutre et distributivité) } \\
\text { note: } 0.5\end{array}$ & $\begin{array}{l}\text { 1: Utilisation des } \\
\text { théorèmes de } \\
\text { l'élément neutre }\end{array}$ & $\begin{array}{l}\text { 13: Théorème de la } \\
\text { distributivité de la } \\
\text { somme sur le produit } \\
\text { logique }\end{array}$ \\
\hline 8 & $\begin{array}{l}\text { 3: L'apprenant est } \\
\text { visuel et rapide }\end{array}$ & $\begin{array}{l}\text { 19: Tables de vérité } \\
\text { (complémentation) } \\
\text { note: } 1.0\end{array}$ & $\begin{array}{l}\text { 3: Utilisation des } \\
\text { théorèmes de la } \\
\text { distributivité }\end{array}$ & $\begin{array}{l}\text { 12: Théorème de la } \\
\text { distributivité du produit } \\
\text { sur la somme logique }\end{array}$ \\
\hline 9 & $\begin{array}{l}\text { 9: L'apprenant est } \\
\text { kinesthésique et } \\
\text { rapide }\end{array}$ & $\begin{array}{l}\text { 9: Simplifier (élément } \\
\text { neutre et De Morgan) } \\
\text { note: } 0.5\end{array}$ & $\begin{array}{c}\text { 4: Utilisation des } \\
\text { théorèmes des lois de } \\
\text { De Morgan }\end{array}$ & $\begin{array}{c}\text { 1: Simplification de } \\
\text { l'expression booléenne } \\
\text { donnée }\end{array}$ \\
\hline
\end{tabular}




\begin{tabular}{|c|c|c|c|c|}
\hline 10 & $\begin{array}{c}\text { 9: L'apprenant est } \\
\text { kinesthésique et } \\
\text { rapide }\end{array}$ & $\begin{array}{l}\text { 1: Simplifier } \\
\text { l'expression donnée } \\
\text { note: } 0.0\end{array}$ & $\begin{array}{l}\text { 3: Utilisation des } \\
\text { théorèmes de la } \\
\text { distributivité }\end{array}$ & $\begin{array}{c}\text { 1: Simplification de } \\
\text { l'expression booléenne } \\
\text { donnée }\end{array}$ \\
\hline 11 & $\begin{array}{l}\text { 6: L'apprenant est } \\
\text { auditif et rapide }\end{array}$ & $\begin{array}{c}\text { 1: Simplifier } \\
\text { l'expression donnée } \\
\text { note: } 0.5\end{array}$ & $\begin{array}{l}\text { 1: Utilisation des } \\
\text { théorèmes de } \\
\text { l'élément neutre }\end{array}$ & $\begin{array}{c}\text { 15: La deuxième loi de } \\
\text { De Morgan }\end{array}$ \\
\hline 12 & $\begin{array}{l}\text { 3: L'apprenant est } \\
\text { visuel et rapide }\end{array}$ & $\begin{array}{l}\text { 20: Tables de vérité } \\
\text { (De Morgan) } \\
\text { note: } 1.0\end{array}$ & $\begin{array}{l}\text { 1: Utilisation des } \\
\text { théorèmes de } \\
\text { l'élément neutre }\end{array}$ & $\begin{array}{l}\text { 9: Théorème de } \\
\text { l'élément neutre de la } \\
\text { somme logique }\end{array}$ \\
\hline 13 & $\begin{array}{l}\text { 2: L'apprenant est } \\
\text { visuel et moyen }\end{array}$ & $\begin{array}{l}\text { 18: Tables de vérité } \\
\text { (élément neutre) } \\
\text { note: } 0.5\end{array}$ & $\begin{array}{c}\text { 7: Simplification de } \\
\text { l'expression booléenne } \\
\text { prédéfinie }\end{array}$ & $\begin{array}{l}\text { 9: Théorème de } \\
\text { l'élément neutre de la } \\
\text { somme logique }\end{array}$ \\
\hline 14 & $\begin{array}{l}\text { 3: L'apprenant est } \\
\text { visuel et rapide }\end{array}$ & $\begin{array}{c}\text { 12: Simplifier } \\
\text { (distributivité et De } \\
\text { Morgan) } \\
\text { note: } 0.0\end{array}$ & $\begin{array}{c}\text { 7: Simplification de } \\
\text { l'expression booléenne } \\
\text { prédéfinie }\end{array}$ & $\begin{array}{l}\text { 9: Théorème de } \\
\text { l'élément neutre de la } \\
\text { somme logique }\end{array}$ \\
\hline 15 & $\begin{array}{l}\text { 6: L'apprenant est } \\
\text { auditif et rapide }\end{array}$ & $\begin{array}{l}\text { 15: Simplifier } \\
\text { (distributivité) } \\
\text { note: } 0.5\end{array}$ & $\begin{array}{l}\text { 5: Simplification } \\
\text { d'expressions } \\
\text { booléennes à } 2 \\
\text { variables }\end{array}$ & $\begin{array}{l}\text { 13: Théorème de la } \\
\text { distributivité de la } \\
\text { somme sur le produit } \\
\text { logique }\end{array}$ \\
\hline 16 & $\begin{array}{l}\text { 6: L'apprenant est } \\
\text { auditif et rapide }\end{array}$ & $\begin{array}{l}\text { 6: Simplifier } \\
\text { (complémentation, } \\
\text { distributivité et De } \\
\text { Morgan) } \\
\text { note: } 1.0\end{array}$ & $\begin{array}{l}\text { 5: Simplification } \\
\text { d'expressions } \\
\text { booléennes à } 2 \\
\text { variables }\end{array}$ & $\begin{array}{l}\text { 12: Théorème de la } \\
\text { distributivité du produit } \\
\text { sur la somme logique }\end{array}$ \\
\hline 17 & $\begin{array}{l}\text { 9: L'apprenant est } \\
\text { kinesthésique et } \\
\text { rapide }\end{array}$ & $\begin{array}{l}\text { 2: Simplifier (ensemble } \\
\text { des théorèmes) } \\
\text { note: } 0.5\end{array}$ & $\begin{array}{l}\text { 5: Simplification } \\
\text { d'expressions } \\
\text { booléennes à } 2 \\
\text { variables }\end{array}$ & $\begin{array}{l}\text { 10: Théorème de la } \\
\text { complémentation du } \\
\text { produit logique }\end{array}$ \\
\hline 18 & $\begin{array}{l}\text { 9: L'apprenant est } \\
\text { kinesthésique et } \\
\text { rapide }\end{array}$ & $\begin{array}{l}\text { 31: Introduction à } \\
\text { l'algèbre de Boole pour } \\
\text { visuels } \\
\text { note: } 0.0\end{array}$ & $\begin{array}{l}\text { 5: Simplification } \\
\text { d'expressions } \\
\text { booléennes à } 2 \\
\text { variables }\end{array}$ & $\begin{array}{l}\text { 11: Théorème de la } \\
\text { complémentation de la } \\
\text { somme logique }\end{array}$ \\
\hline 19 & $\begin{array}{l}\text { 6: L'apprenant est } \\
\text { auditif et rapide }\end{array}$ & $\begin{array}{l}\text { 32: Introduction à } \\
\text { l'algèbre de Boole pour } \\
\text { auditifs } \\
\text { note: } 0.5\end{array}$ & $\begin{array}{c}\text { 4: Utilisation des } \\
\text { théorèmes des lois de } \\
\text { De Morgan }\end{array}$ & $\begin{array}{c}\text { 1: Simplification de } \\
\text { l'expression booléenne } \\
\text { donnée }\end{array}$ \\
\hline 20 & $\begin{array}{l}\text { 6: L'apprenant est } \\
\text { auditif et rapide }\end{array}$ & $\begin{array}{l}\text { 1: Simplifier } \\
\text { l'expression donnée } \\
\text { note: } 1.0\end{array}$ & $\begin{array}{l}\text { 8: Utilisation des } \\
\text { postulats }\end{array}$ & \\
\hline
\end{tabular}

Réussite de l'objectif final. 
Ce deuxième cas d'étudiant moyen et instable montre comment le comportement de PROLIN varie d'une fois à l'autre. La différence avec le cas 3 se situe uniquement dans le choix des éléments de chacune des bases que fait PROLIN. Ceci est dû au fait qu'il fait certains choix de façon aléatoire, un peu comme le professeur qui ne sait pas quelle est la meilleure à adopter. 


\section{Cas 5:}

Profil simulé: Instabilité moins fine que les précédentes.

Pourcentage visuel: $\quad 0.8$

Pourcentage auditif: $\quad 0.2$

Pourcentage kinesthésique: $\quad 0.0$

Vitesse d'apprentissage: $\quad 0.8$

\begin{tabular}{|c|c|c|c|c|}
\hline & CONTEXTE & ACTIVITE & RESULTAT & PROGRAMME \\
\hline 1 & $\begin{array}{l}\text { 3: L'apprenant est } \\
\text { visuel et rapide }\end{array}$ & $\begin{array}{c}\text { 30: Compléter des } \\
\text { tables de vérité sur les } \\
\text { postulats } \\
\text { note: } 0.8\end{array}$ & $\begin{array}{l}\text { 1: Utilisation des } \\
\text { théorèmes de } \\
\text { l'élément neutre }\end{array}$ & $\begin{array}{l}\text { 2: Introduction à } \\
\text { l'algèbre de Boole }\end{array}$ \\
\hline 2 & $\begin{array}{l}\text { 7: L'apprenant est } \\
\text { kinesthésique et lent }\end{array}$ & $\begin{array}{c}\text { 26: Résoudre } \\
\text { (distributivité du } \\
\text { produit sur la somme } \\
\text { logique) } \\
\text { note: } 0.4\end{array}$ & $\begin{array}{l}\text { 1: Utilisation des } \\
\text { théorèmes de } \\
\text { l'élément neutre }\end{array}$ & $\begin{array}{l}\text { 2: Introduction à } \\
\text { l'algèbre de Boole }\end{array}$ \\
\hline 3 & $\begin{array}{l}\text { 7: L'apprenant est } \\
\text { kinesthésique et lent }\end{array}$ & $\begin{array}{c}\text { 29: Résoudre } \\
\text { (deuxième loi de De } \\
\text { Morgan) } \\
\text { note: } 0.0\end{array}$ & $\begin{array}{l}\text { 3: Utilisation des } \\
\text { théorèmes de la } \\
\text { distributivité }\end{array}$ & $\begin{array}{l}\text { 8: Théorème de } \\
\text { l'élément neutre du } \\
\text { produit logique }\end{array}$ \\
\hline 4 & $\begin{array}{l}\text { 3: L'apprenant est } \\
\text { visuel et rapide }\end{array}$ & $\begin{array}{l}\text { 22: Résoudre (élément } \\
\text { neutre du produit } \\
\text { logique) } \\
\text { note: } 0.4\end{array}$ & $\begin{array}{l}\text { 3: Utilisation des } \\
\text { théorèmes de la } \\
\text { distributivité }\end{array}$ & $\begin{array}{l}\text { 8: Théorème de } \\
\text { l'élément neutre du } \\
\text { produit logique }\end{array}$ \\
\hline 5 & $\begin{array}{l}\text { 6: L'apprenant est } \\
\text { auditif et rapide }\end{array}$ & $\begin{array}{l}\text { 4: Simplifier (élément } \\
\text { neutre, } \\
\text { complémentation et } \\
\text { De Morgan) } \\
\text { note: } 0.6\end{array}$ & $\begin{array}{l}\text { 2: Utilisation des } \\
\text { théorèmes de la } \\
\text { complémentation }\end{array}$ & $\begin{array}{c}\text { 1: Simplification de } \\
\text { l'expression booléenne } \\
\text { donnée }\end{array}$ \\
\hline 6 & $\begin{array}{l}\text { 9: L'apprenant est } \\
\text { kinesthésique et } \\
\text { rapide }\end{array}$ & $\begin{array}{l}\text { 1: Simplifier } \\
\text { l'expression donnée } \\
\text { note: } 1.0\end{array}$ & $\begin{array}{c}\text { 8: Utilisation des } \\
\text { postulats }\end{array}$ & \\
\hline
\end{tabular}

Réussite de l'objectif final. 
Cet autre cas d'instabilité donne des résultats à peu près similaires aux précédents. PROLIN est donc aussi embarrassé et propose dès l'étape 6 à l'activité finale que l'usager réussi par hasard. 


\section{Cas 6:}

Profil simulé: Etudiant faible défini comme étant uniquement visuel. Cet étudiant ne comprendra la théorie de l'algèbre de Boole qu'après avoir fait l'activité 31.

Pourcentage visuel: $\quad 1.0$

Pourcentage auditif: $\quad 0.0$

Pourcentage kinesthésique: $\quad 0.0$

Vitesse d'apprentissage: $\quad 0.4$

\begin{tabular}{|c|c|c|c|c|}
\hline & CONTEXTE & ACTIVITE & RESULTAT & PROGRAMME \\
\hline 1 & $\begin{array}{l}\text { 3: L'apprenant est } \\
\text { visuel et rapide }\end{array}$ & $\begin{array}{l}\text { 30: Compléter des } \\
\text { tables de vérité sur les } \\
\text { postulats } \\
\text { note: } 0.3\end{array}$ & $\begin{array}{l}\text { 7: Simplification de } \\
\text { l'expression booléenne } \\
\text { prédéfinie }\end{array}$ & $\begin{array}{l}\text { 3: Simplification } \\
\text { d'expressions } \\
\text { booléennes }\end{array}$ \\
\hline 2 & $\begin{array}{l}\text { 3: L'apprenant est } \\
\text { visuel et rapide }\end{array}$ & $\begin{array}{l}17: \text { Compléter des } \\
\text { énoncés de théorèmes } \\
\text { note: } 0.0\end{array}$ & $\begin{array}{c}\text { 7: Simplification de } \\
\text { l'expression booléenne } \\
\text { prédéfinie }\end{array}$ & $\begin{array}{c}\text { 9: Théorème de } \\
\text { l'élément neutre de la } \\
\text { somme logique }\end{array}$ \\
\hline 3 & $\begin{array}{l}\text { 2: L'apprenant est } \\
\text { visuel et moyen }\end{array}$ & $\begin{array}{l}\text { 18: Tables de vérité } \\
\text { (élément neutre) } \\
\text { note: } 0.2\end{array}$ & $\begin{array}{c}\text { 8: Utilisation des } \\
\text { postulats }\end{array}$ & $\begin{array}{c}\text { 3: Simplification } \\
\text { d'expressions } \\
\text { booléennes }\end{array}$ \\
\hline 4 & $\begin{array}{l}\text { 2: L'apprenant est } \\
\text { visuel et moyen }\end{array}$ & $\begin{array}{l}\text { 21: Tables de vérité } \\
\text { (lois de De Morgan) } \\
\text { note: } 0.2\end{array}$ & $\begin{array}{c}\text { 4: Utilisation des } \\
\text { théorèmes des lois de } \\
\text { De Morgan }\end{array}$ & $\begin{array}{c}\text { 14: La première loi de } \\
\text { De Morgan }\end{array}$ \\
\hline 5 & $\begin{array}{l}\text { 6: L'apprenant est } \\
\text { auditif et rapide }\end{array}$ & $\begin{array}{c}\text { 28: Résoudre } \\
\text { (première loi de De } \\
\text { Morgan) } \\
\text { note: } 0.25 \\
\end{array}$ & $\begin{array}{l}\text { 4: Utilisation des } \\
\text { théorèmes des lois de } \\
\text { De Morgan }\end{array}$ & $\begin{array}{l}\text { 2: Introduction à } \\
\text { l'algèbre de Boole }\end{array}$ \\
\hline 6 & $\begin{array}{l}\text { 1: L'apprenant est } \\
\text { visuel et lent }\end{array}$ & $\begin{array}{c}\text { 23: Résoudre (élément } \\
\text { neutre de la somme } \\
\text { logique) } \\
\text { note: } 0.6\end{array}$ & $\begin{array}{l}\text { 1: Utilisation des } \\
\text { théorèmes de } \\
\text { l'élément neutre }\end{array}$ & $\begin{array}{l}\text { 11: Théorème de la } \\
\text { complémentation de la } \\
\text { somme logique }\end{array}$ \\
\hline 7 & $\begin{array}{l}\text { 2: L'apprenant est } \\
\text { visuel et moyen }\end{array}$ & $\begin{array}{l}\text { 19: Tables de vérité } \\
\text { (complémentation) } \\
\text { note: } 0.0\end{array}$ & $\begin{array}{l}\text { 1: Utilisation des } \\
\text { théorèmes de } \\
\text { l'élément neutre }\end{array}$ & \begin{tabular}{|c|} 
1: Simplification de \\
l'expression booléenne \\
donnée
\end{tabular} \\
\hline 8 & $\begin{array}{l}\text { 6: L'apprenant est } \\
\text { auditif et rapide }\end{array}$ & $\begin{array}{c}\text { 1: Simplifier } \\
\text { l'expression donnée } \\
\text { note: } 0.0\end{array}$ & $\begin{array}{c}\text { 4: Utilisation des } \\
\text { théorèmes des lois de } \\
\text { De Morgan }\end{array}$ & $\begin{array}{c}\text { 8: Théorème de } \\
\text { l'élément neutre du } \\
\text { produit logique }\end{array}$ \\
\hline 9 & $\begin{array}{c}\text { 9: L'apprenant est } \\
\text { kinesthésique et } \\
\text { rapide }\end{array}$ & $\begin{array}{l}\text { 1: Simplifier } \\
\text { l'expression donnée } \\
\text { note: } 0.0\end{array}$ & $\begin{array}{c}\text { 4: Utilisation des } \\
\text { théorèmes des lois de } \\
\text { De Morgan }\end{array}$ & $\begin{array}{c}\text { 8: Théorème de } \\
\text { l'élément neutre du } \\
\text { produit logique }\end{array}$ \\
\hline
\end{tabular}




\begin{tabular}{|c|c|c|c|c|}
\hline 10 & $\begin{array}{l}\text { 9: L'apprenant est } \\
\text { kinesthésique et } \\
\text { rapide }\end{array}$ & $\begin{array}{l}\text { 22: Résoudre (élément } \\
\text { neutre du produit } \\
\text { logique) } \\
\text { note: } 0.6\end{array}$ & $\begin{array}{c}\text { 4: Utilisation des } \\
\text { théorèmes des lois de } \\
\text { De Morgan }\end{array}$ & $\begin{array}{l}\text { 8: Théorème de } \\
\text { l'élément neutre du } \\
\text { produit logique }\end{array}$ \\
\hline 11 & $\begin{array}{c}\text { 9: L'apprenant est } \\
\text { kinesthésique et } \\
\text { rapide }\end{array}$ & $\begin{array}{c}\text { 12: Simplifier } \\
\text { (distributivité et De } \\
\text { Morgan) } \\
\text { note: } 0.0\end{array}$ & $\begin{array}{l}\text { 2: Utilisation des } \\
\text { théorèmes de la } \\
\text { complémentation }\end{array}$ & $\begin{array}{l}\text { 10: Théorème de la } \\
\text { complémentation du } \\
\text { produit logique }\end{array}$ \\
\hline 12 & $\begin{array}{l}\text { 3: L'apprenant est } \\
\text { visuel et rapide }\end{array}$ & $\begin{array}{c}\text { 10: Simplifier } \\
\text { (complémentation et } \\
\text { distributivité) } \\
\text { note: } 0.0\end{array}$ & $\begin{array}{l}\text { 2: Utilisation des } \\
\text { théorèmes de la } \\
\text { complémentation }\end{array}$ & $\begin{array}{l}\text { 3: Simplification } \\
\text { d'expressions } \\
\text { booléennes }\end{array}$ \\
\hline 13 & $\begin{array}{l}\text { 6: L'apprenant est } \\
\text { auditif et rapide }\end{array}$ & $\begin{array}{l}\text { 2: Simplifier (ensemble } \\
\text { des théorèmes) } \\
\text { note: } 0.0\end{array}$ & $\begin{array}{l}\text { 2: Utilisation des } \\
\text { théorèmes de la } \\
\text { complémentation }\end{array}$ & $\begin{array}{c}\text { 1: Simplification de } \\
\text { l'expression booléenne } \\
\text { donnée }\end{array}$ \\
\hline 14 & $\begin{array}{l}\text { 9: L'apprenant est } \\
\text { kinesthésique et } \\
\text { rapide }\end{array}$ & $\begin{array}{c}\text { 1: Simplifier } \\
\text { l'expression donnée } \\
\text { note: } 0.7\end{array}$ & $\begin{array}{l}\text { 1: Utilisation des } \\
\text { théorèmes de } \\
\text { l'élément neutre }\end{array}$ & $\begin{array}{l}\text { 2: Introduction à } \\
\text { l'algèbre de Boole }\end{array}$ \\
\hline 15 & $\begin{array}{l}\text { 9: L'apprenant est } \\
\text { kinesthésique et } \\
\text { rapide }\end{array}$ & $\begin{array}{l}\text { 8: Simplifier (élément } \\
\text { neutre et distributivité) } \\
\text { note: } 0.0\end{array}$ & $\begin{array}{l}\text { 5: Simplification } \\
\text { d'expressions } \\
\text { booléennes à } 2 \\
\text { variables }\end{array}$ & $\begin{array}{c}\text { 1: Simplification de } \\
\text { l'expression booléenne } \\
\text { donnée }\end{array}$ \\
\hline 16 & $\begin{array}{l}\text { 3: L'apprenant est } \\
\text { visuel et rapide }\end{array}$ & $\begin{array}{l}\text { 1: Simplifier } \\
\text { l'expression donnée } \\
0.7\end{array}$ & $\begin{array}{l}\text { 5: Simplification } \\
\text { d'expressions } \\
\text { booléennes à } 2 \\
\text { variables }\end{array}$ & $\begin{array}{c}\text { 11: Théorème de la } \\
\text { complémentation de la } \\
\text { somme logique }\end{array}$ \\
\hline 17 & $\begin{array}{l}\text { 6: L'apprenant est } \\
\text { auditif et rapide }\end{array}$ & \begin{tabular}{|c|}
$31:$ Introduction à \\
l'algèbre de Boole pour \\
visuels \\
note: 1.0
\end{tabular} & $\begin{array}{l}\text { 1: Utilisation des } \\
\text { théorèmes de } \\
\text { l'élément neutre }\end{array}$ & $\begin{array}{l}\text { 10: Théorème de la } \\
\text { complémentation du } \\
\text { produit logique }\end{array}$ \\
\hline 18 & $\begin{array}{l}\text { 3: L'apprenant est } \\
\text { visuel et rapide }\end{array}$ & $\begin{array}{c}\text { 5: Simplifier (élément } \\
\text { neutre, distributivité et } \\
\text { De Morgan) } \\
\text { note: } 0.7\end{array}$ & $\begin{array}{l}\text { 3: Utilisation des } \\
\text { théorèmes de la } \\
\text { distributivité }\end{array}$ & $\begin{array}{l}\text { 9: Théorème de } \\
\text { l'élément neutre de la } \\
\text { somme logique }\end{array}$ \\
\hline 19 & $\begin{array}{l}\text { 9: L'apprenant est } \\
\text { kinesthésique et } \\
\text { rapide }\end{array}$ & $\begin{array}{l}\text { 33: Introduction à } \\
\text { l'algèbre de Boole pour } \\
\text { kinesthésiques) } \\
\text { note: } 1.0\end{array}$ & $\begin{array}{c}\text { 4: Utilisation des } \\
\text { théorèmes des lois de } \\
\text { De Morgan }\end{array}$ & $\begin{array}{c}\text { 14: La première loi de } \\
\text { De Morgan }\end{array}$ \\
\hline 20 & $\begin{array}{l}\text { 3: L'apprenant est } \\
\text { visuel et rapide }\end{array}$ & $\begin{array}{l}\text { 11: Simplifier } \\
\text { (complémentation et } \\
\text { De Morgan) } \\
\text { note: } 1.0\end{array}$ & $\begin{array}{l}\text { 5: Simplification } \\
\text { d'expressions } \\
\text { booléennes à } 2 \\
\text { variables }\end{array}$ & $\begin{array}{c}\text { 15: La deuxième loi de } \\
\text { De Morgan }\end{array}$ \\
\hline 21 & $\begin{array}{l}\text { 9: L'apprenant est } \\
\text { kinesthésique et } \\
\text { rapide }\end{array}$ & $\begin{array}{l}\text { 7: Simplifier (élément } \\
\text { neutre et } \\
\text { complémentation) } \\
\text { note: } 1.0\end{array}$ & $\begin{array}{l}\text { 5: Simplification } \\
\text { d'expressions } \\
\text { booléennes à } 2 \\
\text { variables }\end{array}$ & $\begin{array}{c}\text { 6: Les théorèmes de } \\
\text { niveau } 2\end{array}$ \\
\hline
\end{tabular}




\begin{tabular}{|c|c|c|c|c|}
\hline 22 & $\begin{array}{l}\text { 6: L'apprenant est } \\
\text { auditif et rapide }\end{array}$ & $\begin{array}{c}\text { 3: Simplifier (élément } \\
\text { neutre, } \\
\text { complémentation et } \\
\text { distributivité) } \\
\text { note: } 1.0 \\
\end{array}$ & $\begin{array}{l}\text { 5: Simplification } \\
\text { d'expressions } \\
\text { booléennes à } 2 \\
\text { variables }\end{array}$ & $\begin{array}{l}\text { 10: Théorème de la } \\
\text { complémentation du } \\
\text { produit logique }\end{array}$ \\
\hline 23 & $\begin{array}{l}\text { 9: L'apprenant est } \\
\text { kinesthésique et } \\
\text { rapide }\end{array}$ & $\begin{array}{c}\text { 6: Simplifier } \\
\text { (complémentation, } \\
\text { distributivité et De } \\
\text { Morgan) } \\
\text { note: } 1.0\end{array}$ & $\begin{array}{l}\text { 5: Simplification } \\
\text { d'expressions } \\
\text { booléennes à } 2 \\
\text { variables }\end{array}$ & $\begin{array}{l}\text { 11: Théorème de la } \\
\text { complémentation de la } \\
\text { somme logique }\end{array}$ \\
\hline 24 & $\begin{array}{l}\text { 3: L'apprenant est } \\
\text { visuel et rapide }\end{array}$ & $\begin{array}{c}\text { 32: Introduction à } \\
\text { l'algèbre de Boole pour } \\
\text { auditifs } \\
\text { note: } 1.0 \\
\end{array}$ & $\begin{array}{l}\text { 2: Utilisation des } \\
\text { théorèmes de la } \\
\text { complémentation }\end{array}$ & $\begin{array}{l}\text { 4: Ensemble des } \\
\text { postulats }\end{array}$ \\
\hline 25 & $\begin{array}{l}\text { 7: L'apprenant est } \\
\text { kinesthésique et lent }\end{array}$ & $\begin{array}{l}\text { 25: Résoudre } \\
\text { (distributivité du } \\
\text { produit sur la somme } \\
\text { logique) } \\
\text { note: } 1.0\end{array}$ & $\begin{array}{c}\text { 7: Simplification de } \\
\text { l'expression booléenne } \\
\text { prédéfinie }\end{array}$ & $\begin{array}{c}\text { 5: Les théorèmes de } \\
\text { niveau } 1\end{array}$ \\
\hline 26 & $\begin{array}{l}\text { 3: L'apprenant est } \\
\text { visuel et rapide }\end{array}$ & $\begin{array}{l}\text { 9: Simplifier (élément } \\
\text { neutre et De Morgan) } \\
\text { note: } 1.0\end{array}$ & $\begin{array}{c}\text { 5: Simplification } \\
\text { d'expressions } \\
\text { booléennes à } 2 \\
\text { variables } \\
\end{array}$ & $\begin{array}{c}\text { 7: Les théorèmes de } \\
\text { niveau } 3\end{array}$ \\
\hline 27 & $\begin{array}{l}\text { 9: L'apprenant est } \\
\text { kinesthésique et } \\
\text { rapide }\end{array}$ & $\begin{array}{l}\text { 4: Simplifier (élément } \\
\text { neutre, } \\
\text { complémentation et } \\
\text { De Morgan) } \\
\text { note: } 1.0 \\
\end{array}$ & $\begin{array}{l}\text { 5: Simplification } \\
\text { d'expressions } \\
\text { booléennes à } 2 \\
\text { variables }\end{array}$ & $\begin{array}{l}\text { 9: Théorème de } \\
\text { l'élément neutre de la } \\
\text { somme logique }\end{array}$ \\
\hline 28 & $\begin{array}{l}\text { 3: L'apprenant est } \\
\text { visuel et rapide }\end{array}$ & $\begin{array}{l}\text { 1: Simplifier } \\
\text { l'expression donnée } \\
\text { note: } 1.0\end{array}$ & $\begin{array}{l}\text { 8: Utilisation des } \\
\text { postulats }\end{array}$ & \\
\hline
\end{tabular}

Réussite de l'objectif final.

Le problème dans la stratégie de PROLIN après la réussite de l'activité 31 à l'étape 17 est dû au fait qu'il ne peut pas répéter les activités déjà faites (sauf l'activité 1). Comme il en avait déjà proposé plusieurs avant, il se trouve limité et on a l'impression qu'il piétine jusqu'à l'étape 26 où il recommence à progresser pour terminer à l'étape $\mathbf{2 8}$ avec l'activité finale.

Ce fait pourrait se corriger en disposant de plus d'activités d'apprentissage. 


\section{Cas 7:}

Profil simulé: Etudiant qui n'a aucune connaissance de l'algèbre de Boole au départ.

Pourcentage visuel:

0.8

Pourcentage auditif:

0.2

Pourcentage kinesthésique:

Vitesse d'apprentissage:

0.2

\begin{tabular}{|c|c|c|c|c|}
\hline & CONTEXTE & ACTIVITE & RESULTAT & PROGRAMME \\
\hline 1 & $\begin{array}{l}\text { 1: L'apprenant est } \\
\text { visuel et lent }\end{array}$ & $\begin{array}{c}\text { 31: Introduction à } \\
\text { l'algèbre de Boole pour } \\
\text { visuels } \\
\text { note: } 1.0\end{array}$ & $\begin{array}{l}\text { 8: Utilisation des } \\
\text { postulats }\end{array}$ & $\begin{array}{l}\text { 3: Simplification } \\
\text { d'expressions } \\
\text { booléennes }\end{array}$ \\
\hline 2 & $\begin{array}{l}\text { 9: L'apprenant est } \\
\text { kinesthésique et } \\
\text { rapide }\end{array}$ & $\begin{array}{l}\text { 5: Simplifier (élément } \\
\text { neutre, distributivité et } \\
\text { De Morgan) } \\
\text { note: } 0.6\end{array}$ & $\begin{array}{l}\text { 1: Utilisation des } \\
\text { théorèmes de } \\
\text { l'élément neutre }\end{array}$ & $\begin{array}{l}\text { 3: Simplification } \\
\text { d'expressions } \\
\text { booléennes }\end{array}$ \\
\hline 3 & $\begin{array}{l}\text { 3: L'apprenant est } \\
\text { visuel et rapide }\end{array}$ & $\begin{array}{l}\text { 2: Simplifier (ensemble } \\
\text { des théorèmes) } \\
\text { note: } 0.3\end{array}$ & $\begin{array}{c}\text { 4: Utilisation des } \\
\text { théorèmes des lois de } \\
\text { De Morgan }\end{array}$ & \begin{tabular}{|c|} 
1: Simplification de \\
l'expression booléenne \\
donnée
\end{tabular} \\
\hline 4 & $\begin{array}{l}\text { 6: L'apprenant est } \\
\text { auditif et rapide }\end{array}$ & $\begin{array}{l}\text { 1: Simplifier } \\
\text { l'expression donnée } \\
\text { note: } 0.0\end{array}$ & $\begin{array}{l}\text { 3: Utilisation des } \\
\text { théorèmes de la } \\
\text { distributivité }\end{array}$ & $\begin{array}{c}\text { 1: Simplification de } \\
\text { l'expression booléenne } \\
\text { donnée }\end{array}$ \\
\hline 5 & $\begin{array}{l}\text { 3: L'apprenant est } \\
\text { visuel et rapide }\end{array}$ & $\begin{array}{l}\text { 1: Simplifier } \\
\text { l'expression donnée } \\
\text { note: } 0.7\end{array}$ & $\begin{array}{l}\text { 1: Utilisation des } \\
\text { théorèmes de } \\
\text { l'élément neutre }\end{array}$ & $\begin{array}{c}11: \text { Théorème de la } \\
\text { complémentation de la } \\
\text { somme logique }\end{array}$ \\
\hline 6 & $\begin{array}{l}\text { 3: L'apprenant est } \\
\text { visuel et rapide }\end{array}$ & $\begin{array}{l}\text { 8: Simplifier (élément } \\
\text { neutre et De Morgan) } \\
\text { note: } 0.65\end{array}$ & $\begin{array}{c}\text { 5: Simplification } \\
\text { d'expressions } \\
\text { booléennes à } 2 \\
\text { variables } \\
\end{array}$ & $\begin{array}{l}\text { 8: Théorème de } \\
\text { l'élément neutre du } \\
\text { produit logique }\end{array}$ \\
\hline 7 & $\begin{array}{l}\text { 3: L'apprenant est } \\
\text { visuel et rapide }\end{array}$ & $\begin{array}{l}\text { 19: Tables de vérité } \\
\text { (complémentation) } \\
\text { note: } 1.0\end{array}$ & $\begin{array}{l}\text { 2: Utilisation des } \\
\text { théorèmes de la } \\
\text { complémentation }\end{array}$ & $\begin{array}{l}\text { 12: Théorème de la } \\
\text { distributivité du produit } \\
\text { sur la somme logique }\end{array}$ \\
\hline 8 & $\begin{array}{l}\text { 9: L'apprenant est } \\
\text { kinesthésique et } \\
\text { rapide }\end{array}$ & $\begin{array}{c}\text { 11: Simplifier } \\
\text { (complémentation et } \\
\text { De Morgan) } \\
\text { note: } 1.0\end{array}$ & $\begin{array}{l}\text { 5: Simplification } \\
\text { d'expressions } \\
\text { booléennes à } 2 \\
\text { variables } \\
\end{array}$ & $\begin{array}{c}\text { 14: La première loi de } \\
\text { De Morgan }\end{array}$ \\
\hline 9 & $\begin{array}{l}\text { 3: L'apprenant est } \\
\text { visuel et rapide }\end{array}$ & $\begin{array}{c}\text { 12: Simplifier } \\
\text { (distributivité et } \mathrm{De} \\
\text { Morgan) } \\
\text { note: } 1.0\end{array}$ & $\begin{array}{c}\text { 5: Simplification } \\
\text { d'expressions } \\
\text { booléennes à } 2 \\
\text { variables }\end{array}$ & $\begin{array}{c}\text { 15: La deuxième loi de } \\
\text { De Morgan }\end{array}$ \\
\hline
\end{tabular}




\begin{tabular}{|c|c|c|c|c|}
\hline 10 & $\begin{array}{l}\text { 9: L'apprenant est } \\
\text { kinesthésique et } \\
\text { rapide }\end{array}$ & $\begin{array}{l}\text { 9: Simplifier (élément } \\
\text { neutre et De Morgan) } \\
\text { note: } 1.0\end{array}$ & $\begin{array}{l}\text { 5: Simplification } \\
\text { d'expressions } \\
\text { booléennes à } 2 \\
\text { variables }\end{array}$ & $\begin{array}{l}\text { 8: Théorème de } \\
\text { l'élément neutre du } \\
\text { produit logique }\end{array}$ \\
\hline 11 & $\begin{array}{l}\text { 3: L'apprenant est } \\
\text { visuel et rapide }\end{array}$ & $\begin{array}{l}\text { 21: Tables de vérité } \\
\text { (lois de De Morgan) } \\
\text { note: } 1.0\end{array}$ & $\begin{array}{l}\text { 1: Utilisation des } \\
\text { théorèmes de } \\
\text { l'élément neutre }\end{array}$ & $\begin{array}{c}\text { 11: Théorème de la } \\
\text { complémentation de la } \\
\text { somme logique }\end{array}$ \\
\hline 12 & $\begin{array}{l}\text { 9: L'apprenant est } \\
\text { kinesthésique et } \\
\text { rapide }\end{array}$ & $\begin{array}{l}\text { 18: Tables de vérité } \\
\text { (élément neutre) } \\
\text { note: } 1.0\end{array}$ & $\begin{array}{c}\text { 4: Utilisation des } \\
\text { théorèmes des lois de } \\
\text { De Morgan }\end{array}$ & $\begin{array}{l}\text { 2: Introduction à } \\
\text { l'algèbre de Boole }\end{array}$ \\
\hline 13 & $\begin{array}{l}\text { 3: L'apprenant est } \\
\text { visuel et rapide }\end{array}$ & $\begin{array}{l}\text { 4: Simplifier (élément } \\
\text { neutre, } \\
\text { complémentation et } \\
\text { De Morgan) } \\
\text { note: } 1.0\end{array}$ & $\begin{array}{l}\text { 5: Simplification } \\
\text { d'expressions } \\
\text { booléennes à } 2 \\
\text { variables }\end{array}$ & $\begin{array}{l}\text { 8: Théorème de } \\
\text { l'élément neutre du } \\
\text { produit logique }\end{array}$ \\
\hline 14 & $\begin{array}{l}\text { 6: L'apprenant est } \\
\text { auditif et rapide }\end{array}$ & $\begin{array}{l}\text { 33: Introduction à } \\
\text { l'algèbre de Boole pour } \\
\text { kinesthésiques } \\
\text { note: } 1.0\end{array}$ & $\begin{array}{l}\text { 1: Utilisation des } \\
\text { théorèmes de } \\
\text { l'élément neutre }\end{array}$ & $\begin{array}{l}\text { 9: Théorème de } \\
\text { l'élément neutre de la } \\
\text { somme logique }\end{array}$ \\
\hline 15 & $\begin{array}{l}\text { 3: L'apprenant est } \\
\text { visuel et rapide }\end{array}$ & $\begin{array}{l}\text { 7: Simplifier (élément } \\
\text { neutre et } \\
\text { complémentation) } \\
\text { note: } 1.0\end{array}$ & $\begin{array}{l}\text { 5: Simplification } \\
\text { d'expressions } \\
\text { booléennes à } 2 \\
\text { variables }\end{array}$ & $\begin{array}{c}\text { 6: Les théorèmes de } \\
\text { niveau } 2\end{array}$ \\
\hline 16 & $\begin{array}{c}\text { 9: L'apprenant est } \\
\text { kinesthésique et } \\
\text { rapide }\end{array}$ & $\begin{array}{l}\text { 30: Compléter des } \\
\text { tables de vérité sur les } \\
\text { postulats } \\
\text { note: } 1.0\end{array}$ & $\begin{array}{l}\text { 5: Simplification } \\
\text { d'expressions } \\
\text { booléennes à } 2 \\
\text { variables }\end{array}$ & $\begin{array}{l}\text { 10: Théorème de la } \\
\text { complémentation du } \\
\text { produit logique }\end{array}$ \\
\hline 17 & $\begin{array}{l}\text { 3: L'apprenant est } \\
\text { visuel et rapide }\end{array}$ & $\begin{array}{l}\text { 10: Simplifier } \\
\text { (complémentation et } \\
\text { distributivité) } \\
\text { note: } 1.0\end{array}$ & $\begin{array}{l}\text { 5: Simplification } \\
\text { d'expressions } \\
\text { booléennes à } 2 \\
\text { variables }\end{array}$ & $\begin{array}{l}\text { 13: Théorème de la } \\
\text { distributivité de la } \\
\text { somme sur le produit } \\
\text { logique }\end{array}$ \\
\hline 18 & $\begin{array}{l}\text { 3: L'apprenant est } \\
\text { visuel et rapide }\end{array}$ & $\begin{array}{l}\text { 3: Simplifier (élément } \\
\text { neutre, } \\
\text { complémentation et } \\
\text { distributivité) } \\
\text { note: } 1.0\end{array}$ & $\begin{array}{l}\text { 5: Simplification } \\
\text { d'expressions } \\
\text { booléennes à } 2 \\
\text { variables }\end{array}$ & $\begin{array}{l}\text { 11: Théorème de la } \\
\text { complémentation de la } \\
\text { somme logique }\end{array}$ \\
\hline 19 & $\begin{array}{l}\text { 9: L'apprenant est } \\
\text { kinesthésique et } \\
\text { rapide }\end{array}$ & $\begin{array}{c}\text { 6: Simplifier } \\
\text { (complémentation, } \\
\text { distributivité et De } \\
\text { Morgan) } \\
\text { note: } 1.0\end{array}$ & $\begin{array}{l}\text { 5: Simplification } \\
\text { d'expressions } \\
\text { booléennes à } 2 \\
\text { variables }\end{array}$ & $\begin{array}{l}\text { 13: Théorème de la } \\
\text { distributivité de la } \\
\text { somme sur le produit } \\
\text { logique }\end{array}$ \\
\hline 20 & $\begin{array}{l}\text { 6: L'apprenant est } \\
\text { auditif et rapide }\end{array}$ & $\begin{array}{l}\text { 1: Simplifier } \\
\text { l'expression donnée } \\
\text { note: } 1.0\end{array}$ & $\begin{array}{l}\text { 8: Utilisation des } \\
\text { postulats }\end{array}$ & \\
\hline
\end{tabular}

Réussite de l'objectif final. 
On voit ici que pour un étudiant n'ayant aucune connaissance de l'algèbre de Boole au départ, on a avantage à le définir très lent (vitesse d'apprentissage $=0.2$ ). De cette façon, PROLIN progressera lentement en variant le type d'activités proposées. II amène ainsi lentement mais sûrement l'apprenant vers l'objectif visé qu'il atteint à la vingtième étape. 


\section{Cas 8:}

Profil simulé: Etudiant qui est défini lent mais qui s'avère être rapide.

Pourcentage visuel: $\quad 0.8$

Pourcentage auditif: $\quad 0.2$

Pourcentage kinesthésique: $\quad 0.0$

Vitesse d'apprentissage: $\quad 0.2$

\begin{tabular}{c|c|c|c|c} 
& CONTEXTE & ACTIVITE & RESULTAT & PROGRAMME \\
\hline \hline 1 & $\begin{array}{c}\text { 1: L'apprenant est } \\
\text { visuel et lent }\end{array}$ & $\begin{array}{c}\text { 31: Introduction à } \\
\text { l'algèbre de Boole pour } \\
\text { visuels } \\
\text { note: } 1.0\end{array}$ & $\begin{array}{c}\text { 8: Utilisation des } \\
\text { postulats }\end{array}$ & $\begin{array}{c}\text { 3: Simplification } \\
\text { d'expressions } \\
\text { booléennes }\end{array}$ \\
\hline 2 & $\begin{array}{c}\text { 9: L'apprenant est } \\
\text { kinesthésique et } \\
\text { rapide }\end{array}$ & $\begin{array}{c}5: \text { Simplifier (élément } \\
\text { neutre, distributivité et } \\
\text { De Morgan) } \\
\text { note: } 1.0\end{array}$ & $\begin{array}{c}\text { 7: Simplification de } \\
\text { 'expression booléenne } \\
\text { prédéfinie }\end{array}$ & $\begin{array}{c}\text { 1: Simplification de } \\
\text { l'expression booléenne } \\
\text { donnée }\end{array}$ \\
\hline 3 & $\begin{array}{c}\text { 3: L'apprenant est } \\
\text { visuel et rapide }\end{array}$ & $\begin{array}{c}1: \text { Simplifier } \\
\text { l'expression donnée }\end{array}$ & $\begin{array}{c}\text { 8: Utilisation des } \\
\text { postulats }\end{array}$ & \\
\hline
\end{tabular}

Réussite de l'objectif final.

Si un étudiant est défini lent mais est rapide, PROLIN est en difficulté. II lui offrira donc rapidement l'activité finale puisqu'il ne comprend pas ce comportement en désaccord avec sa connaissance de l'apprenant. 


\section{Cas 9:}

Profil simulé: Etudiant rapide bien défini.

Pourcentage visuel: $\quad 0.8$

Pourcentage auditif: $\quad 0.2$

Pourcentage kinesthésique: $\quad 0.0$

Vitesse d'apprentissage: $\quad 1.0$

\begin{tabular}{|c|c|c|c|c|}
\hline & CONTEXTE & ACTIVITE & RESULTAT & PROGRAMME \\
\hline 1 & $\begin{array}{l}\text { 3: L'apprenant est } \\
\text { visuel et rapide }\end{array}$ & $\begin{array}{l}\text { 30: Compléter des } \\
\text { tables de vérité sur les } \\
\text { postulats } \\
\text { note: } 1.0\end{array}$ & $\begin{array}{l}\text { 1: Utilisation des } \\
\text { théorèmes de } \\
\text { l'élément neutre }\end{array}$ & $\begin{array}{l}\text { 2: Introduction à } \\
\text { l'algèbre de Boole }\end{array}$ \\
\hline 2 & $\begin{array}{l}\text { 7: L'apprenant est } \\
\text { kinesthésique et lent }\end{array}$ & $\begin{array}{c}\text { 26: Résoudre } \\
\text { (distributivité du } \\
\text { produit sur la somme } \\
\text { logique) } \\
\text { note: } 1.0\end{array}$ & $\begin{array}{l}\text { 3: Utilisation des } \\
\text { théorèmes de la } \\
\text { distributivité }\end{array}$ & $\begin{array}{l}\text { 13: Théorème de la } \\
\text { distributivité de la } \\
\text { somme sur le produit } \\
\text { logique }\end{array}$ \\
\hline 3 & $\begin{array}{l}\text { 3: L'apprenant est } \\
\text { visuel et rapide }\end{array}$ & $\begin{array}{l}\text { 27: Résoudre } \\
\text { (distributivité de la } \\
\text { somme sur le produit } \\
\text { logique) } \\
\text { note: } 1.0\end{array}$ & $\begin{array}{l}\text { 3: Utilisation des } \\
\text { théorèmes de la } \\
\text { distributivité }\end{array}$ & $\begin{array}{l}\text { 12: Théorème de la } \\
\text { distributivité du produit } \\
\text { sur la somme logique }\end{array}$ \\
\hline 4 & $\begin{array}{l}\text { 3: L'apprenant est } \\
\text { visuel et rapide }\end{array}$ & $\begin{array}{l}\text { 11: Simplifier } \\
\text { (complémentation et } \\
\text { De Morgan) } \\
\text { note: } 1.0\end{array}$ & $\begin{array}{l}\text { 2: Utilisation des } \\
\text { théorèmes de la } \\
\text { complémentation }\end{array}$ & $\begin{array}{c}\text { 14: La première loi de } \\
\text { De Morgan }\end{array}$ \\
\hline 5 & $\begin{array}{l}\text { 6: L'apprenant est } \\
\text { auditif et rapide }\end{array}$ & $\begin{array}{l}\text { 8: Simplifier (élément } \\
\text { neutre et distributivité) } \\
\text { note: } 1.0\end{array}$ & $\begin{array}{c}\text { 5: Simplification } \\
\text { d'expressions } \\
\text { booléennes à } 2 \\
\text { variables }\end{array}$ & $\begin{array}{c}\text { 6: Les théorèmes de } \\
\text { niveau } 2\end{array}$ \\
\hline 6 & $\begin{array}{l}\text { 9: L'apprenant est } \\
\text { kinesthésique et } \\
\text { rapide }\end{array}$ & $\begin{array}{l}\text { 3: Simplifier (élément } \\
\text { neutre, } \\
\text { complémentation et } \\
\text { distributivité) } \\
\text { note: } 1.0\end{array}$ & $\begin{array}{l}\text { 5: Simplification } \\
\text { d'expressions } \\
\text { booléennes à } 2 \\
\text { variables }\end{array}$ & $\begin{array}{l}\text { 1: Simplification de } \\
\text { l'expression booléenne } \\
\text { donnée }\end{array}$ \\
\hline 7 & $\begin{array}{l}\text { 2: L'apprenant est } \\
\text { visuel et moyen }\end{array}$ & $\begin{array}{l}\text { 1: Simplifier } \\
\text { l'expression donnée } \\
\text { note: } 1.0\end{array}$ & $\begin{array}{l}\text { 8: Utilisation des } \\
\text { postulats }\end{array}$ & \\
\hline
\end{tabular}

Réussite de l'objectif final. 
Si un étudiant rapide est bien défini, la progression des activités est correcte. PROLIN offre une suite d'activités en augmentant régulièrement le niveau de difficulté jusqu'à la fin. 


\section{Cas 10:}

Profil simulé: Etudiant défini faible qui obtient des résultats nuls partout.

Pourcentage visuel: $\quad 0.8$

Pourcentage auditif: $\quad 0.2$

Pourcentage kinesthésique: $\quad 0.0$

Vitesse d'apprentissage: $\quad 0.2$

\begin{tabular}{|c|c|c|c|c|}
\hline & CONTEXTE & ACTIVITE & RESULTAT & PROGRAMME \\
\hline 1 & $\begin{array}{l}\text { 1: L'apprenant est } \\
\text { visuel et lent }\end{array}$ & $\begin{array}{c}\text { 31: Introduction à } \\
\text { l'algèbre de Boole pour } \\
\text { visuels } \\
\text { note: } 0.0\end{array}$ & $\begin{array}{l}\text { 8: Utilisation des } \\
\text { postulats }\end{array}$ & $\begin{array}{l}\text { 3: Simplification } \\
\text { d'expressions } \\
\text { booléennes }\end{array}$ \\
\hline 2 & $\begin{array}{c}\text { 8: L'apprenant est } \\
\text { kinesthésique et } \\
\text { moyen }\end{array}$ & $\begin{array}{l}\text { 30: Compléter des } \\
\text { tables de vérité sur les } \\
\text { postulats } \\
\text { note: } 0.0\end{array}$ & $\begin{array}{l}\text { 2: Utilisation des } \\
\text { théorèmes de la } \\
\text { complémentation }\end{array}$ & $\begin{array}{l}\text { 10: Théorème de la } \\
\text { complémentation du } \\
\text { produit logique }\end{array}$ \\
\hline 3 & $\begin{array}{l}\text { 2: L'apprenant est } \\
\text { visuel et moyen }\end{array}$ & $\begin{array}{l}\text { 19: Tables de vérité } \\
\text { (complémentation) } \\
\text { note: } 0.0\end{array}$ & $\begin{array}{l}\text { 2: Utilisation des } \\
\text { théorèmes de la } \\
\text { complémentation }\end{array}$ & $\begin{array}{l}\text { 2: Introduction à } \\
\text { l'algèbre de Boole }\end{array}$ \\
\hline 4 & $\begin{array}{l}\text { 1: L'apprenant est } \\
\text { visuel et lent }\end{array}$ & $\begin{array}{c}\text { 24: Résoudre } \\
\text { (complémentation du } \\
\text { produit logique) } \\
\text { note: } 0.0\end{array}$ & $\begin{array}{l}\text { 3: Utilisation des } \\
\text { théorèmes de la } \\
\text { distributivité }\end{array}$ & $\begin{array}{l}\text { 10: Théorème de la } \\
\text { complémentation du } \\
\text { produit logique }\end{array}$ \\
\hline 5 & $\begin{array}{l}\text { 3: L'apprenant est } \\
\text { visuel et rapide }\end{array}$ & $\begin{array}{l}\text { 9: Simplifier (élément } \\
\text { neutre et De Morgan) } \\
\text { note: } 0.0\end{array}$ & $\begin{array}{l}\text { 4: Utilisation des } \\
\text { théorèmes des lois de } \\
\text { De Morgan }\end{array}$ & $\begin{array}{c}\text { 13: Théorème de la } \\
\text { distributivité de la } \\
\text { somme sur le produit } \\
\text { logique }\end{array}$ \\
\hline 6 & $\begin{array}{c}\text { 9: L'apprenant est } \\
\text { kinesthésique et } \\
\text { rapide }\end{array}$ & $\begin{array}{l}\text { 8: Simplifier (élément } \\
\text { neutre et distributivité) } \\
\text { note: } 0.0\end{array}$ & $\begin{array}{l}\text { 1: Utilisation des } \\
\text { théorèmes de } \\
\text { l'élément neutre }\end{array}$ & $\begin{array}{c}\text { 11: Théorème de la } \\
\text { complémentation de la } \\
\text { somme logique }\end{array}$ \\
\hline 7 & $\begin{array}{l}\text { 2: L'apprenant est } \\
\text { visuel et moyen }\end{array}$ & $\begin{array}{l}\text { 17: Compléter des } \\
\text { énoncés de théorèmes } \\
\text { note: } 0.0\end{array}$ & $\begin{array}{l}\text { 5: Simplification } \\
\text { d'expressions } \\
\text { booléennes à } 2 \\
\text { variables }\end{array}$ & $\begin{array}{l}\text { 11: Théorème de la } \\
\text { complémentation de la } \\
\text { somme logique }\end{array}$ \\
\hline 8 & $\begin{array}{l}\text { 6: L'apprenant est } \\
\text { auditif et rapide }\end{array}$ & $\begin{array}{c}\text { 11: Simplifier } \\
\text { (complémentation et } \\
\text { De Morgan) } \\
\text { note: } 0.0\end{array}$ & $\begin{array}{l}\text { 5: Simplification } \\
\text { d'expressions } \\
\text { booléennes à } 2 \\
\text { variables }\end{array}$ & $\begin{array}{l}\text { 10: Théorème de la } \\
\text { complémentation du } \\
\text { produit logique }\end{array}$ \\
\hline$\overline{9}$ & $\begin{array}{l}\text { 3: L'apprenant est } \\
\text { visuel et rapide }\end{array}$ & $\begin{array}{l}\text { 16: Simplifier (lois de } \\
\text { De Morgan) } \\
\text { note: } 0.0\end{array}$ & $\begin{array}{l}\text { 5: Simplification } \\
\text { d'expressions } \\
\text { booléennes à } 2 \\
\text { variables }\end{array}$ & $\begin{array}{c}\text { 1: Simplification de } \\
\text { l'expression booléenne } \\
\text { donnée }\end{array}$ \\
\hline
\end{tabular}




\begin{tabular}{|c|c|c|c|c|}
\hline 10 & $\begin{array}{l}\text { 9: L'apprenant est } \\
\text { kinesthésique et } \\
\text { rapide }\end{array}$ & $\begin{array}{l}\text { 1: Simplifier } \\
\text { l'expression donnée } \\
\text { note: } 0.0\end{array}$ & $\begin{array}{l}\text { 5: Simplification } \\
\text { d'expressions } \\
\text { booléennes à } 2 \\
\text { variables }\end{array}$ & $\begin{array}{l}\text { 1: Simplification de } \\
\text { l'expression booléenne } \\
\text { donnée }\end{array}$ \\
\hline 11 & $\begin{array}{l}\text { 6: L'apprenant est } \\
\text { auditif et rapide }\end{array}$ & $\begin{array}{l}1: \text { Simplifier } \\
\text { l'expression donnée } \\
\text { note: } 0.7\end{array}$ & $\begin{array}{l}\text { 2: Utilisation des } \\
\text { théorèmes de la } \\
\text { complémentation }\end{array}$ & $\begin{array}{l}\text { 12: Théorème de la } \\
\text { distributivité du produit } \\
\text { sur la somme logique }\end{array}$ \\
\hline 12 & $\begin{array}{l}\text { 6: L'apprenant est } \\
\text { auditif et rapide }\end{array}$ & $\begin{array}{c}\text { 12: Simplifier } \\
\text { (distributivité et De } \\
\text { Morgan) } \\
\text { note: } 0.0\end{array}$ & $\begin{array}{l}\text { 1: Utilisation des } \\
\text { théorèmes de } \\
\text { l'élément neutre }\end{array}$ & $\begin{array}{l}\text { 9: Théorème de } \\
\text { l'élément neutre de la } \\
\text { somme logique }\end{array}$ \\
\hline 13 & $\begin{array}{l}\text { 2: L'apprenant est } \\
\text { visuel et moyen }\end{array}$ & $\begin{array}{c}\text { 23: Résoudre (élément } \\
\text { neutre de la somme } \\
\text { logique) } \\
\text { note: } 0.0\end{array}$ & $\begin{array}{l}\text { 4: Utilisation des } \\
\text { thérèmes des lois de } \\
\text { De Morgan }\end{array}$ & $\begin{array}{c}\text { 15: La deuxième loi de } \\
\text { De Morgan }\end{array}$ \\
\hline 14 & $\begin{array}{l}\text { 3: L'apprenant est } \\
\text { visuel et rapide }\end{array}$ & $\begin{array}{l}\text { 10: Simplifier } \\
\text { (complémentation et } \\
\text { distributivité) } \\
\text { note: } 0.0\end{array}$ & $\begin{array}{l}\text { 3: Utilisation des } \\
\text { théorèmes de la } \\
\text { distributivité }\end{array}$ & $\begin{array}{l}\text { 3: Simplification } \\
\text { d'expressions } \\
\text { booléennes }\end{array}$ \\
\hline 15 & $\begin{array}{l}\text { 9: L'apprenant est } \\
\text { kinesthésique et } \\
\text { rapide }\end{array}$ & $\begin{array}{l}\text { 2: Simplifier (ensemble } \\
\text { des théorèmes) } \\
\text { note: } 0.0\end{array}$ & $\begin{array}{l}\text { 6: Simplification } \\
\text { d'expressions } \\
\text { booléennes à } 3 \\
\text { variables }\end{array}$ & $\begin{array}{c}\text { 15: La deuxième loi de } \\
\text { De Morgan }\end{array}$ \\
\hline 16 & $\begin{array}{l}\text { 6: L'apprenant est } \\
\text { auditif et rapide }\end{array}$ & $\begin{array}{l}\text { 18: Tables de vérité } \\
\text { (élément neutre) } \\
\text { note: } 0.0\end{array}$ & $\begin{array}{l}\text { 5: Simplification } \\
\text { d'expressions } \\
\text { booléennes à } 2 \\
\text { variables } \\
\end{array}$ & $\begin{array}{c}\text { 15: La deuxième loi de } \\
\text { De Morgan }\end{array}$ \\
\hline 17 & $\begin{array}{l}\text { 3: L'apprenant est } \\
\text { visuel et rapide }\end{array}$ & $\begin{array}{l}\text { 6: Simplifier } \\
\text { (complémentation, } \\
\text { distributivité et De } \\
\text { Morgan) } \\
\text { note: } 0.0\end{array}$ & $\begin{array}{l}\text { 5: Simplification } \\
\text { d'expressions } \\
\text { booléennes à } 2 \\
\text { variables }\end{array}$ & $\begin{array}{l}\text { 14: La première loi de } \\
\text { De Morgan }\end{array}$ \\
\hline 18 & $\begin{array}{c}\text { 9: L'apprenant est } \\
\text { kinesthésique et } \\
\text { rapide }\end{array}$ & $\begin{array}{c}1: \text { Simplifier } \\
\text { l'expression donnée } \\
\text { note: } 0.7\end{array}$ & $\begin{array}{l}\text { 1: Utilisation des } \\
\text { théorèmes de } \\
\text { l'élément neutre }\end{array}$ & $\begin{array}{c}\text { 1: Simplification de } \\
\text { l'expression booléenne } \\
\text { donnée }\end{array}$ \\
\hline 19 & $\begin{array}{l}\text { 6: L'apprenant est } \\
\text { auditif et rapide }\end{array}$ & $\begin{array}{l}\text { 7: Simplifier (élément } \\
\text { neutre et De Morgan) } \\
\text { note: } 0.0\end{array}$ & $\begin{array}{c}\text { 4: Utilisation des } \\
\text { théorèmes des lois de } \\
\text { De Morgan }\end{array}$ & $\begin{array}{l}\text { 9: Théorème de } \\
\text { l'élément neutre de la } \\
\text { somme logique }\end{array}$ \\
\hline 20 & $\begin{array}{l}\text { 9: L'apprenant est } \\
\text { kinesthésique et } \\
\text { rapide }\end{array}$ & $\begin{array}{l}\text { 3: Simplifier (élément } \\
\text { neutre, } \\
\text { complémentation et } \\
\text { distributivité) } \\
\text { note: } 0.0\end{array}$ & $\begin{array}{l}\text { 3: Utilisation des } \\
\text { théorèmes de la } \\
\text { distributivité }\end{array}$ & $\begin{array}{l}\text { 9: Théorème de } \\
\text { l'élément neutre de la } \\
\text { somme logique }\end{array}$ \\
\hline 21 & $\begin{array}{l}\text { 3: L'apprenant est } \\
\text { visuel et rapide }\end{array}$ & $\begin{array}{l}\text { 21: Tables de vérité } \\
\text { (lois de De Morgan) } \\
\text { note: } 0.0\end{array}$ & $\begin{array}{l}\text { 5: Simplification } \\
\text { d'expressions } \\
\text { booléennes à } 2 \\
\text { variables } \\
\end{array}$ & $\begin{array}{l}\text { 13: Théorème de la } \\
\text { distributivité de la } \\
\text { somme sur le produit } \\
\text { logique }\end{array}$ \\
\hline
\end{tabular}




\begin{tabular}{|c|c|c|c|c|}
\hline 22 & $\begin{array}{l}\text { 6: L'apprenant est } \\
\text { auditif et rapide }\end{array}$ & $\begin{array}{c}\text { 14: Simplifier } \\
\text { (complémentation) } \\
\text { note: } 0.0\end{array}$ & $\begin{array}{l}\text { 5: Simplification } \\
\text { d'expressions } \\
\text { booléennes à } 2 \\
\text { variables }\end{array}$ & $\begin{array}{c}\text { 14: La première loi de } \\
\text { De Morgan }\end{array}$ \\
\hline 23 & $\begin{array}{c}\text { 9: L'apprenant est } \\
\text { kinesthésique et } \\
\text { rapide }\end{array}$ & $\begin{array}{l}\text { 13: Simplifier (élément } \\
\text { neutre) } \\
\text { note: } 0.0\end{array}$ & $\begin{array}{c}\text { 5: Simplification } \\
\text { d'expressions } \\
\text { booléennes à } 2 \\
\text { variables }\end{array}$ & $\begin{array}{l}\text { 14: La première loi de } \\
\text { De Morgan }\end{array}$ \\
\hline 24 & $\begin{array}{l}\text { 3: L'apprenant est } \\
\text { visuel et rapide }\end{array}$ & $\begin{array}{c}\text { 32: Introduction à } \\
\text { l'algèbre de Boole pour } \\
\text { auditifs } \\
\text { note: } 0.0\end{array}$ & $\begin{array}{c}\text { 6: Simplification } \\
\text { d'expressions } \\
\text { booléennes à } 3 \\
\text { variables }\end{array}$ & $\begin{array}{c}\text { 6: Les théorèmes de } \\
\text { niveau } 2\end{array}$ \\
\hline 25 & $\begin{array}{l}\text { 6: L'apprenant est } \\
\text { auditif et rapide }\end{array}$ & $\begin{array}{l}\text { 4: Simplifier (élément } \\
\text { neutre, } \\
\text { complémentation et } \\
\text { De Morgan) } \\
\text { note: } 0.0\end{array}$ & $\begin{array}{l}\text { 6: Simplification } \\
\text { d'expressions } \\
\text { booléennes à } 3 \\
\text { variables }\end{array}$ & $\begin{array}{c}\text { 7: Les théorèmes de } \\
\text { niveau } 3\end{array}$ \\
\hline 26 & $\begin{array}{l}\text { 9: L'apprenant est } \\
\text { kinesthésique et } \\
\text { rapide }\end{array}$ & $\begin{array}{c}\text { 5: Simplifier (élément } \\
\text { neutre, distributivité et } \\
\text { De Morgan) } \\
\text { note: } 0.0\end{array}$ & $\begin{array}{l}\text { 6: Simplification } \\
\text { d'expressions } \\
\text { booléennes à } 3 \\
\text { variables } \\
\end{array}$ & $\begin{array}{c}\text { 5: Les théorèmes de } \\
\text { niveau } 1\end{array}$ \\
\hline 27 & $\begin{array}{l}\text { 3: L'apprenant est } \\
\text { visuel et rapide }\end{array}$ & $\begin{array}{r}\text { 15: Simplifier } \\
\text { (distributivité) } \\
\text { note: } 0.0\end{array}$ & $\begin{array}{l}\text { 5: Simplification } \\
\text { d'expressions } \\
\text { booléennes à } 2 \\
\text { variables }\end{array}$ & $\begin{array}{l}\text { 8: Théorème de } \\
\text { l'élément neutre du } \\
\text { produit logique }\end{array}$ \\
\hline 28 & $\begin{array}{l}\text { 6: L'apprenant est } \\
\text { auditif et rapide }\end{array}$ & $\begin{array}{l}\text { 33: Introduction à } \\
\text { l'algèbre de Boole pour } \\
\text { kinesthésiques } \\
\text { note: } 0.0\end{array}$ & $\begin{array}{l}\text { 5: Simṕlification } \\
\text { d'expressions } \\
\text { booléennes à } 2 \\
\text { variables }\end{array}$ & $\begin{array}{l}\text { 12: Théorème de la } \\
\text { distributivité du produit } \\
\text { sur la somme logique }\end{array}$ \\
\hline 29 & $\begin{array}{c}\text { 9: L'apprenant est } \\
\text { kinesthésique et } \\
\text { rapide }\end{array}$ & $\begin{array}{l}\text { 1: Simplifier } \\
\text { l'expression donnée } \\
\text { note: } 0.8\end{array}$ & $\begin{array}{l}\text { 8: Utilisation des } \\
\text { postulats }\end{array}$ & $\begin{array}{l}\text { 2: Introduction à } \\
\text { l'algèbre de Boole }\end{array}$ \\
\hline 30 & $\begin{array}{l}\text { 9: L'apprenant est } \\
\text { kinesthésique et } \\
\text { rapide }\end{array}$ & $\begin{array}{l}\text { aucune } \\
\text { Niveau } 2\end{array}$ & & \\
\hline 31 & $\begin{array}{l}\text { 9: L'apprenant est } \\
\text { kinesthésique et } \\
\text { rapide }\end{array}$ & $\begin{array}{l}\text { 1: Simplifier } \\
\text { l'expression donnée } \\
\text { note: } 1.0\end{array}$ & $\begin{array}{l}\text { 1: Utilisation des } \\
\text { théorèmes de } \\
\text { l'élément neutre }\end{array}$ & \\
\hline
\end{tabular}

Réussite de l'objectif final.

On a un étudiant faible qui obtient des résultats nuls partout. PROLIN offre tout ce qu'il a à offrir dans le but d'aider l'apprenant à comprendre. Finalement, 
PROLIN ne trouve plus d'activités compatibles et va au niveau 2 à l'étape 30 . II proposera ensuite une dernière fois l'activité finale.

Si l'apprenant n'avait pas encore réussit l'activité finale, PROLIN aurait continuer à offrir des activités (en aurait même répété). Si l'impasse avait perduré, PROLIN se serait rendu à l'évidence d'un échec en passant au niveau 3 sans réussite de l'objectif final. 


\section{Cas 11:}

Profil simulé: Etudiant présentant des difficultés avec les lois de De Morgan. II apprend toutefois en cours de route.

Pourcentage visuel:

0.8

Pourcentage auditif:

0.2

Pourcentage kinesthésique:

0.0

Vitesse d'apprentissage:

0.8

\begin{tabular}{|c|c|c|c|c|}
\hline & CONTEXTE & ACTIVITE & RESULTAT & PROGRAMME \\
\hline 1 & $\begin{array}{l}\text { 6: L'apprenant est } \\
\text { auditif et rapide }\end{array}$ & $\begin{array}{c}\text { 30: Compléter des } \\
\text { tables de vérité sur les } \\
\text { postulats } \\
\text { note: } 1.0\end{array}$ & $\begin{array}{l}\text { 1: Utilisation des } \\
\text { théorèmes de } \\
\text { l'élément neutre }\end{array}$ & $\begin{array}{c}\text { 2: Introduction à } \\
\text { l'algèbre de Boole }\end{array}$ \\
\hline 2 & $\begin{array}{l}\text { 7: L'apprenant est } \\
\text { kinesthésique et lent }\end{array}$ & $\begin{array}{l}\text { 26: Résoudre } \\
\text { (distributivité du } \\
\text { produit sur la somme } \\
\text { logique) } \\
\text { note: } 1.0\end{array}$ & $\begin{array}{l}\text { 3: Utilisation des } \\
\text { théorèmes de la } \\
\text { distributivité }\end{array}$ & $\begin{array}{l}\text { 13: Théorème de la } \\
\text { distributivité de la } \\
\text { somme sur le produit } \\
\text { logique }\end{array}$ \\
\hline 3 & $\begin{array}{l}\text { 6: L'apprenant est } \\
\text { auditif et rapide }\end{array}$ & $\begin{array}{c}\text { 27: Résoudre } \\
\text { (distributivité de la } \\
\text { somme sur le produit } \\
\text { logique) } \\
\text { note: } 1.0\end{array}$ & $\begin{array}{l}\text { 3: Utilisation des } \\
\text { théorèmes de la } \\
\text { distributivité }\end{array}$ & $\begin{array}{l}\text { 12: Théorème de la } \\
\text { distributivité du produit } \\
\text { sur la somme logique }\end{array}$ \\
\hline 4 & $\begin{array}{l}\text { 6: L'apprenant est } \\
\text { auditif et rapide }\end{array}$ & $\begin{array}{c}\text { 11: Simplifier } \\
\text { (complémentation et } \\
\text { De Morgan) } \\
\text { note: } 0.6\end{array}$ & $\begin{array}{l}\text { 2: Utilisation des } \\
\text { théorèmes de la } \\
\text { complémentation }\end{array}$ & $\begin{array}{c}\text { 14: La première loi de } \\
\text { De Morgan }\end{array}$ \\
\hline 5 & $\begin{array}{l}\text { 9: L'apprenant est } \\
\text { kinesthésique et } \\
\text { rapide }\end{array}$ & $\begin{array}{l}\text { 18: Tables de vérité } \\
\text { (élément neutre) } \\
\text { note: } 1.0\end{array}$ & $\begin{array}{l}\text { 3: Utilisation des } \\
\text { théorèmes de la } \\
\text { distributivité }\end{array}$ & $\begin{array}{c}\text { 14: La première loi de } \\
\text { De Morgan }\end{array}$ \\
\hline 6 & $\begin{array}{l}\text { 6: L'apprenant est } \\
\text { auditif et rapide }\end{array}$ & $\begin{array}{c}\text { 4: Simplifier (élément } \\
\text { neutre, } \\
\text { complémentation et } \\
\text { De Morgan) } \\
\text { note: } 1.0\end{array}$ & $\begin{array}{c}\text { 4: Utilisation des } \\
\text { théorèmes des lois de } \\
\text { De Morgan }\end{array}$ & $\begin{array}{l}\text { 1: Simplification de } \\
\text { l'expression booléenne } \\
\text { donnée }\end{array}$ \\
\hline 7 & $\begin{array}{l}\text { 3: L'apprenant est } \\
\text { visuel et rapide }\end{array}$ & $\begin{array}{l}\text { 1: Simplifier } \\
\text { l'expression donnée } \\
\text { note: } 0.0\end{array}$ & $\begin{array}{l}\text { 3: Utilisation des } \\
\text { théorèmes de la } \\
\text { distributivité }\end{array}$ & $\begin{array}{l}\text { 13: Théorème de la } \\
\text { distributivité de la } \\
\text { somme sur le produit } \\
\text { logique }\end{array}$ \\
\hline
\end{tabular}




\begin{tabular}{|c|c|c|c|c|}
\hline 8 & $\begin{array}{l}\text { 6: L'apprenant est } \\
\text { auditif et rapide }\end{array}$ & $\begin{array}{l}\text { 10: Simplifier } \\
\text { (complémentation et } \\
\text { distributivité) } \\
\text { note: } 1.0\end{array}$ & $\begin{array}{l}\text { 5: Simplification } \\
\text { d'expressions } \\
\text { booléennes à } 2 \\
\text { variables }\end{array}$ & $\begin{array}{c}\text { 6: Les théorèmes de } \\
\text { niveau } 2\end{array}$ \\
\hline 9 & $\begin{array}{l}\text { 9: L'apprenant est } \\
\text { kinesthésique et } \\
\text { rapide }\end{array}$ & $\begin{array}{l}\text { 3: Simplifier (élément } \\
\text { neutre, } \\
\text { complémentation et } \\
\text { distributivité) } \\
\text { note: } 1.0\end{array}$ & $\begin{array}{l}5: \text { Simplification } \\
\text { d'expressions } \\
\text { booléennes à } 2 \\
\text { variables }\end{array}$ & $\begin{array}{l}\text { 13: Théorème de la } \\
\text { distributivité de la } \\
\text { somme sur le produit } \\
\text { logique }\end{array}$ \\
\hline 10 & $\begin{array}{l}\text { 6: L'apprenant est } \\
\text { auditif et rapide }\end{array}$ & $\begin{array}{l}\text { 6: Simplifier } \\
\text { (complémentation, } \\
\text { distributivité et De } \\
\text { Morgan) } \\
\text { note: } 0.4\end{array}$ & $\begin{array}{l}\text { 4: Utilisation des lois } \\
\text { de De Morgan }\end{array}$ & $\begin{array}{c}\text { 1: Simplification de } \\
\text { l'expression booléenne } \\
\text { donnée }\end{array}$ \\
\hline 11 & $\begin{array}{l}\text { 3: L'apprenant est } \\
\text { visuel et rapide }\end{array}$ & $\begin{array}{l}\text { 1: Simplifier } \\
\text { l'expression donnée } \\
\text { note: } 0.0\end{array}$ & $\begin{array}{l}\text { 1: Utilisation des } \\
\text { théorèmes de } \\
\text { l'élément neutre }\end{array}$ & $\begin{array}{l}\text { 3: Simplification } \\
\text { d'expressions } \\
\text { booléennes }\end{array}$ \\
\hline 12 & $\begin{array}{l}\text { 6: L'apprenant est } \\
\text { auditif et rapide }\end{array}$ & $\begin{array}{l}\text { 2: Simplifier (ensemble } \\
\text { des théorèmes) } \\
\text { note: } 0.7\end{array}$ & $\begin{array}{l}\text { 5: Simplification } \\
\text { d'expressions } \\
\text { booléennes à } 2 \\
\text { variables }\end{array}$ & $\begin{array}{l}\text { 9: Théorème de } \\
\text { l'élément neutre de la } \\
\text { somme logique }\end{array}$ \\
\hline 13 & $\begin{array}{l}\text { 9: L'apprenant est } \\
\text { kinesthésique et } \\
\text { rapide }\end{array}$ & $\begin{array}{l}\text { 1: Simplifier } \\
\text { l'expression donnée } \\
\text { note: } 1.0\end{array}$ & $\begin{array}{l}\text { 8: Utilisation des } \\
\text { postulats }\end{array}$ & \\
\hline
\end{tabular}

Réussite de l'objectif final.

Ce dernier cas en est un typique pour ce type d'apprentissage. II s'agit d'un étudiant qui présente des difficultés avec les lois de De Morgan mais non avec les autres théorèmes. Il échoue la première activité où il a à se servir des lois de De Morgan à l'étape 4. A chaque fois qu'il a besoin de ces lois par la suite (aux étapes $6,7,10,11$ et 12), il réussit un peu mieux en s'améliorant à chaque fois. C'est ainsi qu'il finit par réussir l'objectif final à l'étape 13.

Remarque: Le lecteur trouvera l'impression complète tel qu'obtenue directement par le logiciel PROLIN pour ce cas à la partie 6 du présent rapport technique. 
PARTIE 6

\section{SORTIE D'IMPRIMANTE}




\section{PARTIE 6}

\section{SORTIE D'IMPRIMANTE}

Ce qui suit est une sortie d'imprimante du programme PROLIN. II s'agit en fait du résultat d'une simulation très semblable au cas numéro 11 paraîssant à la partie 5 du présent document. Seulement certains choix d'éléments de contexte les plus compatibles diffèrent parce que PROLIN devait faire certains choix de manière arbitraire. 
contexte le plus compatible est le no $3(0.8)$

Les nouvelles normes calculees pour les activites sont:

Activite $1 \quad 0.1<0.5547002<1$

Activite $20.4<0.5547002<1$

Activite $3 \quad 0.5<0.5547002<0.9$

Activite $4 \quad 0.5<0.5547002<0.9$

Activite $5 \quad 0.5<0.5547002<0.9$

Activite $6 \quad 0.5<0.5547002<0.9$

Activite $7 \quad 0.55<0.5547002<0.85$

Activite $8 \quad 0.55<0.5547002<0.85$

Activite $90.55<0.5547002<0.85$

Aclivite $10 \quad 0.55<0.5547002<0.85$

Activite $110.55<0.5547002<0.85$

Activite $120.55<0.5547002<0.85$

Activite $130.6<0.49923018<0.8$

Activite $140.6<0.49923018<0.8$

Activite $15 \quad 0.6<0.49923018<0.8$

Activite $160.6<0.49923018<0.8$

Activite $170.6<0.5547002<0.8$

Activite $180.6<0.49923018<0.8$

Activite $190.6<0.49923018<0.8$

Activite $200.6<0.49923018<0.8$

Activite $210.6<0.49923018<0.8$

Activite $220.4<0.22188008<0.7$

Activite $230.4<0.22188008<0.7$

Activite $240.4<0.22188008<0.7$

Activite $250.4<0.22188008<0.7$

Activite $260.4<0.22188008<0.7$

Activite $270.4<0.22188008<0.7$

Activite $280.4<0.22188008<0.7$

Activite $290.4<0.22188008<0.7$

Activite $30 \quad 0.2<0.5547002<0.8$

Activite $310.4<0.33282012<0.6$

Activite $320.4<0.33282012<0.6$

Activite $330.4<0.33282012<0.6$

activite le plus compatible est le no $30(0.5547002)$

La note est:1.0

La nouvelle norme pour le contexte choisi est: $0.8+(1.0-0.5)$

Les nouvelles calculees pour les resultats sont:

Resultat $10.3<0.98367977<1$

Resultat $20.3<0.98367977<1$

Resultat $30.3<0.94830405<1$

Resultat $40.3<0.91549413<1$

Resultat $50.3<0.91549413<1.0$

Resultat $60.3<0.83536922<1.0$

Resultat $70.3<0.77968819<0.8$

Resultat $8 \quad 0.3<0.98367977<0.9$

resultat le plus compatible est le no $1(0.98367977)$

Les nouvelles normes calculees pour les programmes sont:

Programme $10.4<0.56097444<1$

Programme $20.4<0.86550229<0.9$

Programme $30.1<0.70020067<0.8$

Programme $40.5<0.64156676<0.8$

Programme 50.6 $<0.75243429<0.8$

Programme $60.6<0.42073083<0.8$

Programme 70.6 $<0.0<0.8$
Programme 80.1 $<0.90384059<0.7$

Programme $90.1<0.90384059<0.7$

Programme $10 \quad 0.1<0.8505901<0.7$

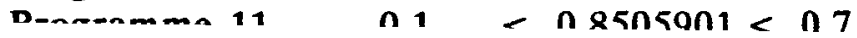


$\begin{array}{lll}\text { Programme 12 } & 0.1 & <0.0<0.7 \\ \text { Programme 13 } & 0.1 & <0.0<0.7 \\ \text { Programme 14 } & 0.1 & <0.0<0.7 \\ \text { Programme 15 } & 0.1 & <0.0<0.7\end{array}$

programme le plus compatible est le no 2(0.86550229)

Les nouvelles normes calculees pour les contextes sont:

$\begin{array}{llll}\text { Contexte } 1 & 0.1 & <0.79429588 & <0.8 \\ \text { Contexte 2 } & 0.2 & <0.62831182 & <0.85 \\ \text { Contexte 3 } & 0.3 & <0.76051083 & <1.0 \\ \text { Contexte 4 } & 0.1 & <0.79429588 & <0.8 \\ \text { Contexte 5 } & 0.2 & <0.62831182 & <0.85 \\ \text { Contexte 6 } & 0.3 & <0.7461907<1.0 \\ \text { Contexte 7 } & 0.1 & <0.79429588 & <0.8 \\ \text { Contexte 8 } & 0.2 & <0.62831182 & <0.85 \\ \text { Contexte 9 } & 0.3 & <0.74584014 & <1.0\end{array}$

contexte le plus compatible est le no 7(0.79429588)

Les nouvelles normes calculees pour les activites sont:

Activite $1 \quad 0.1<0.15430048<1$

Activite $20.4<0.16741166<1$

Activite $3 \quad 0.5<0.20169468<0.9$

Activite $4 \quad 0.5<0.20169468<0.9$

Activite 5 $0.5<0.20169468<0.9$

Activite $6 \quad 0.5<0.20169468<0.9$

Activite $70.55<0.24853747<0.85$

Activite $8 \quad 0.55<0.24853747<0.85$

Activite $90.55<0.24853747<0.85$

Activite $100.55<0.24853747<0.85$

Activite $110.55<0.24853747<0.85$

Activite $120.55<0.24853747 \quad<0.85$

Activite $130.6<0.35317934<0.8$

Activite $140.6<0.35317934<0.8$

Activite $150.6<0.35317934<0.8$

Activite $160.6<0.35317934<0.8$

Activite $17 \quad 0.6<0.59661758<0.8$

Activite $180.6<0.4753612<0.8$

Activite $190.6<0.4753612<0.8$

Activite $20 \quad 0.6<0.4753612<0.8$

Activite $210.6<0.4753612<0.8$

Activite $220.4<0.52322371<0.7$

Activite $230.4<0.52322371<0.7$

Activite $240.4<0.52322371<0.7$

Activite $250.4<0.52322371<0.7$

Activite $260.4<0.52322371<0.7$

Activite $270.4<0.52322371<0.7$

Activite $28 \quad 0.4<0.52322371<0.7$

Activite $290.4<0.52322371<0.7$

Activite $30 \quad 0.7<0.74145221<0.3$

Activite $310.9<0.72543955<0.1$

Activite $320.9<0.72543955<0.1$

Activite $330.9<0.96498344<0.1$

activite le plus compatible est le no $26(0.52322371)$

La note est:1.0

La nouvelle norme pour le contexte choisi est: $0.79429588-(1.0-0.5)$

Les nouvelles calculees pour les resultats sont:
Resultat 1
$0.35<0.59896799$
$<0.95$
Resultat 2
$0.3<0.59896799$
$<1$
Resultat 3
$0.3<0.86090836$
$<1$
Resultat $40.3<0.563972<1$
Resultat $50.3<0.563972<1.0$
Resultat $60.3<0.52342589<1.0$ 
$\begin{array}{llll}\text { Resultat } 7 & 0.3<0.49569345 & <0.8 \\ \text { Resultat } 8 & 0.3 & <0.99489971 & <0.9\end{array}$

resultat le plus compatible est le no 3(0.86090836)

Les nouvelles normes calculees pour les programmes sont:

$\begin{array}{llll}\text { Programme 10.4 } & <0.52239263 & <1 \\ \text { Programme 20.5 } & <0.46316167 & <0.8 \\ \text { Programme 30.1 } & <0.30897803 & <0.8 \\ \text { Programme 40.5 } & <0.06725486 & <0.8 \\ \text { Programme 50.6 } & <0.45902921 & <0.8 \\ \text { Programme 60.6 } & <0.75615022 & <0.8 \\ \text { Programme 70.6 } & <0.59669415 & <0.8 \\ \text { Programme 80.1 } & <0.09474878 & <0.7 \\ \text { Programme 90.1 } & <0.09474878 & <0.7 \\ \text { Programme 10 } & 0.1 & <0.08916658 & <0.7 \\ \text { Programme 11 } & 0.1 & <0.08916658 & <0.7 \\ \text { Programme 12 } & 0.1 & <0.99449025 & <0.7 \\ \text { Programme 13 } & 0.1 & <0.64884015 & <0.7 \\ \text { Programme 14 } & 0.1 & <0.58395614 & <0.7 \\ \text { Programme 15 } & 0.1 & <0.58395614 & <0.7\end{array}$

programme le plus compatible est le no $13(0.64884015)$

Les nouvelles normes calculees pour les contextes sont:

$\begin{array}{llll}\text { Contexte 1 } & 0.1 & <0.49061084 & <0.8 \\ \text { Contexte 2 } & 0.2 & <0.67082631 & <0.85 \\ \text { Contexte 3 } & 0.3 & <0.90536418 & <1.0 \\ \text { Contexte 4 } & 0.1 & <0.49061084 & <0.8 \\ \text { Contexte 5 } & 0.2 & <0.67082631 & <0.85 \\ \text { Contexte 6 } & 0.3 & <0.90331988 & <1.0 \\ \text { Contexte 7 } & 0.1 & <0.38395728 & <0.8 \\ \text { Contexte 8 } & 0.2 & <0.67082631 & <0.85 \\ \text { Contexte 9 } & 0.3 & <0.90327026 & <1.0\end{array}$

contexte le plus compatible est le no $3(0.90536418)$

Les nouvelles normes calculees pour les activites sont:

Activite 1

$0.1<0.75679921$

$<1$

Activite 2

$0.4<0.75718387$

$<1$

$<0.9$

Activite 3

$0.5<0.75833668$

$<0.9$

Activite 4

$0.5<0.75833668$

$<0.9$

Activite 6

$0.5<0.75833668$

$<0.9$

Activite 7

$0.5<0.75833668$

$<0.85$

Activite 8

$0.55<0.76025413$

$<0.85$

Activite 9

$0.55<0.76025413$

$<0.85$

Activite 10

$0.55<0.76025413$

$<0.85$

Activite 11

$0.55<0.76025413$

$<0.85$

Activite 12

$0.55<0.76025413$

$<0.85$

Activite 13

$0.55<0.76025413$

$<0.8$

Activite 14

$0.6<0.69172439$

$<0.8$

Activite 15

$0.6<0.69172439$

$<0.8$

Activite 16

$0.6<0.69172439$

$<0.8$

Activite 17

$0.6<0.69172439$

$<0.8$

Activite 18

$0.6<0.81571993$

$<0.8$

Activite 19

$0.6<0.70175453$

$<0.8$

Activite 20

$0.6<0.70175453$

$<0.8$

Activite 21

$0.6<0.82440533$

$<0.8$

Activite 22

$0.6<0.70175453$

$<0.7$

Activite 23

$0.4<0.35904126$

$<0.7$

Activite 24

$0.4<0.35904126$

$<0.7$

Activite 25

$0.4<0.35904126$

$<0.7$

Activite 26

$0.4<0.35904126$

$<0.2$

Activite 27

$0.9<0.47872669$

$<0.7$

Activite 28

$0.4<0.64914309$

$<0.7$ 
Activite $290.4<0.35904126<0.7$

Activite $30 \quad 0.7<0.83195915<0.3$

Activite $310.9<0.55134984<0.1$

Activite $320.9<0.55134984<0.1$

Activite $330.9<0.59991147<0.1$

activite le plus compatible est le no $27(0.64914309)$

La note est:1.0

La nouvelle norme pour le contexte choisi est: $0.90536418+(1.0-0.5)$

Les nouvelles calculees pour les resultats sont:

$\begin{array}{llll}\text { Resultat 1 } & 0.35 & <0.80492305 & <0.95 \\ \text { Resultat 2 } & 0.3 & <0.80492305 & <1 \\ \text { Resultat 3 } & 0.35<0.93369121 & <0.95 \\ \text { Resultat 4 } & 0.3 & <0.80359871 & <1 \\ \text { Resultat 5 } & 0.3<0.80359871 & <1.0 \\ \text { Resultat 6 } & 0.3 & <0.80216155 & <1.0 \\ \text { Resultat 7 } & 0.3 & <0.80123894 & <0.8 \\ \text { Resultat 8 } & 0.3 & <0.99973369 & <0.9\end{array}$

resultat le plus compatible est le no 3(0.93369121)

Les nouvelles normes calculees pour les programmes sont:

$\begin{array}{llll}\text { Programme 1 0.4 } & <0.54591238 & <1 \\ \text { Programme 20.5 } & <0.47768703 & <0.8 \\ \text { Programme 30.1 } & <0.30965931 & <0.8 \\ \text { Programme 4 0.5 } & <0.0032558<0.8 & \\ \text { Programme 5 0.6 } & <0.43860396 & <0.8 \\ \text { Programme 6 0.6 } & <0.75507712 & <0.8 \\ \text { Programme 7 0.6 } & <0.59999227 & <0.8 \\ \text { Programme 80.1 } & <0.00458678 & <0.7 \\ \text { Programme 9 0.1 } & <0.00458678 & <0.7 & \\ \text { Programme 10 } & 0.1 & <0.00431655 & <0.7 \\ \text { Programme 11 } & 0.1 & <0.00431655 & <0.7 \\ \text { Programme 12 } & 0.1 & <0.68335587 & <0.7 \\ \text { Programme 13 } & 0.2 & <0.99932131 & <0.6 \\ \text { Programme 14 } & 0.1 & <0.61414307 & <0.7 \\ \text { Programme 15 } & 0.1 & <0.61414307 & <0.7\end{array}$

programme le plus compatible est le no 12(0.68335587)

Les nouvelles normes calculees pour les contextes sont:

$\begin{array}{llll}\text { Contexte 1 } & 0.1 & <0.40983819 & <0.8 \\ \text { Contexte 2 } & 0.2 & <0.67595226 & <0.85 \\ \text { Contexte 3 } & 0.3 & <0.99150925 & <1.0 \\ \text { Contexte 4 } & 0.1 & <0.40983819 & <0.8 \\ \text { Contexte 5 } & 0.2 & <0.67595226 & <0.85 \\ \text { Contexte 6 } & 0.3 & <0.93201796 & <1.0 \\ \text { Contexte 7 } & 0.1 & <0.39844486 & <0.8 \\ \text { Contexte 8 } & 0.2 & <0.67595226 & <0.85 \\ \text { Contexte 9 } & 0.3 & <0.93201321 & <1.0\end{array}$

contexte le plus compatible est le no $3(0.99150925)$

Les nouvelles normes calculees pour les activites sont:

Activite $10.1<0.81903196<1$

Activite $20.4<0.81905612<1$

Activite $3 \quad 0.5<0.81912858<0.9$

Activite $4 \quad 0.5<0.81912858<0.9$

Activite $5 \quad 0.5<0.81912858<0.9$

Activite $6 \quad 0.5<0.81912858<0.9$

Activite $7 \quad 0.55<0.81924934<0.85$

Activite $8 \quad 0.55<0.81924934<0.85$

Activite $90.55<0.81924934<0.85$

Activite $10 \quad 0.55<0.81924934<0.85$

Activite $110.55<0.81924934<0.85$

Activite $120.55<0.81924934<0.85$

Activite $130.6<0.73779949<0.8$ 
Activite 14

$$
0.6
$$$$
<0.73779949
$$$$
<0.8
$$

Aclivite 15

$$
0.6
$$$$
<0.73779949
$$$$
<0.8
$$

Aclivite 16

$$
0.6
$$$$
<0.73779949
$$$$
<0.8
$$$$
<0.8
$$

Activite 18

$$
0.6<0.85154794
$$$$
<0.8
$$

Activite 19

$$
0.6<0.73844281
$$$$
<0.8
$$

Activite 20

$$
0.6<0.73844281
$$

$0.6<0.86609244$

$<0.8$

Activite 21

Activite 22

$0.6<0.73844281$

$<0.8$

Activite 23

$0.4<0.33145538$

$<0.7$

Activite 24

$0.4<0.33145538$

$<0.7$

Activite 25

$0.4<0.33145538$

$<0.7$

Activite 26

$0.4<0.33145538$

$0.9<0.64562459$

$<0.7$

Aclivite 27

$$
0.9<0.41115911
$$

$<0.2$

$<0.2$

Activite 28

Activite 29

$0.4<0.33145538$

$0.4<0.33145538$

$<0.7$

$<0.7$

Activite $\mathbf{3 0}$

0.7

$<0.8526148<0.3$

$<0.52454936$

$<0.1$

Activite 31

0.9

$<0.52454936$

$<0.52815797$

$<0.1$

$<0.1$

activite le plus compatible est le no 11(0.81924934)

La note est:0.6

La nouvelle norme pour le contexte choisi est: $0.99150925+(0.6-0.5)$

Les nouvelles calculees pour les resultats sont:

$\begin{array}{llll}\text { Resultat 1 } & 0.35 & <0.87235995 & <0.95 \\ \text { Resultat 2 } & 0.3 & <0.98977253 & <1 \\ \text { Resultat 3 } & 0.4 & <0.87974509 & <0.9 \\ \text { Resultat 4 } & 0.3 & <0.98971033 & <1 \\ \text { Resultat 5 } & 0.3 & <0.94911376 & <1.0 \\ \text { Resultat 6 } & 0.3 & <0.91622321 & <1.0 \\ \text { Resultat 7 } & 0.3 & <0.87216391 & <0.8 \\ \text { Resultat 8 } & 0.3 & <0.99998461 & <0.9\end{array}$

resultat le plus compatible est le no $2(0.98977253)$

Les nouvelles normes calculees pour les programmes sont:

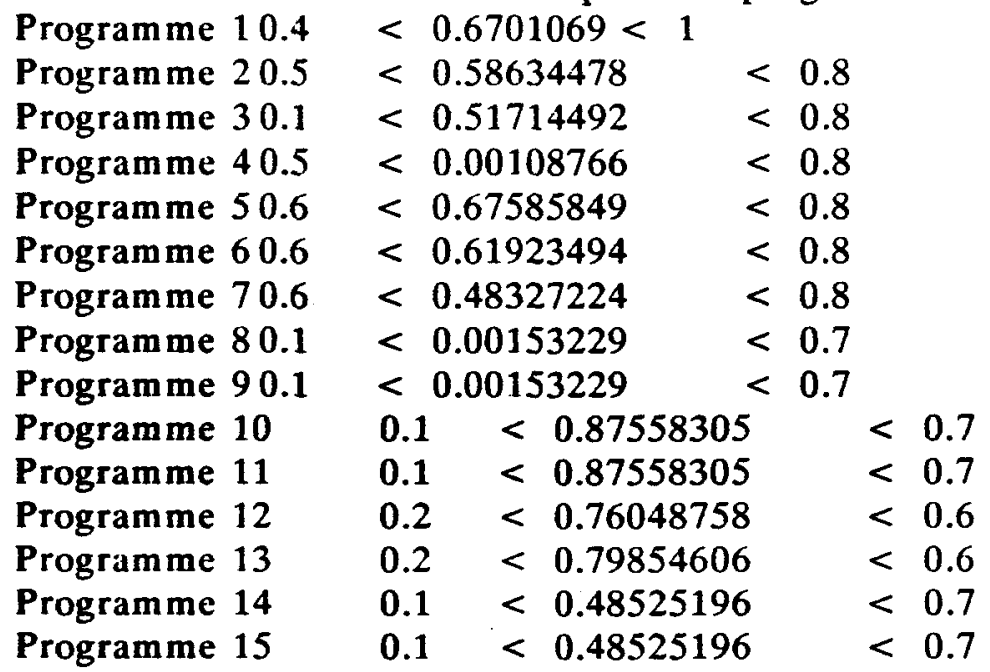

programme le plus compatible est le no 14(0.48525196)

Les nouvelles normes calculees pour les contextes sont:

$\begin{array}{llll}\text { Contexte 1 } & 0.1 & <0.44906913 & <0.8 \\ \text { Contexte 2 } & 0.2 & <0.69561677 & <0.85 \\ \text { Contexte 3 } & 0.3 & <1.0026825<1.0 \\ \text { Contexte 4 } & 0.1 & <0.44906913 & <0.8 \\ \text { Contexte 5 } & 0.2 & <0.69561677 & <0.85 \\ \text { Contexte 6 } & 0.3 & <0.97247415 & <1.0 \\ \text { Contexte 7 } & 0.1 & <0.44716948 & <0.8 \\ \text { Contexte 8 } & 0.2 & <0.69561677 & <0.85 \\ \text { Confexte 9 } & 03 & <007947331 & <1.0\end{array}$


contexte le plus compatible est le no $6(0.97247415)$

Les nouvelles normes calculees pour les activites sont:

Activite 2

$$
\begin{aligned}
& 0.1<0.88020908<1 \\
& 0.4<0.88021356<1 \\
& 0.5<0.880227<0.9
\end{aligned}
$$

Activite 3

Activite 4

Activite 5

$0.5<0.880227<0.9$

Activite 6

$0.5<0.880227<0.9$

Activite 7

$0.5<0.880227<0.9$

Activite 8

$0.55<0.8802494<0.85$

Activite 9

$0.55<0.8802494<0.85$

Activite 10

$0.55<0.8802494<0.85$

Activite 11

$0.55<0.8802494<0.85$

Activite 12

1.05

$0.55<0.8802494<0.85$

Activite 13

Activite 14

$0.6<0.79231262<0.8$

$0.6<0.79231262<0.8$

$<0.35$

Activite 15

Activite 16

0.6

$<0.79231262$

$<0.8$

$0.6<0.79231262<0.8$

Activite 17

0.6

$<0.79231262$

$<0.8$

Activite 18

0.6

$<0.90062988$

$<0.8$

Activite 19

0.6

$<0.79243208$

$<0.8$

Activite 20

0.6

$<0.79243208$

$<0.8$

$0.6<0.81778685<0.8$

Activite 21

Activite 22

0.6

$<0.85446008$

$<0.8$

Activite 23

$0.4<0.3527998<0.7$

Activite 24

$0.4<0.3527998<0.7$

Activite 25

$0.4<0.3527998<0.7$

Activite 26

$0.4<0.3527998<0.7$

Activite 27

0.9

$0.9<0.43087835$

$<0.2$

Activite 28

$0.9<0.36914219$

$0.4<0.53299384$

$<0.2$

Activite 29

$0.4<0.3527998<0.7$

Activite $\mathbf{3 0}$

0.7

$<0.90083105$

$<0.7$

Activite 31

$0.9<0.54771449$

$<0.3$

Activite 32

$0.9<0.54771449$

$<0.1$

Activite 33

$0.9<0.5484053<0.1$

$<0.1$

activite le plus compatible est le no $18(0.79243208)$

La note est:1.0

La nouvelle norme pour le contexte choisi est: $0.97247415+(1.0-0.5)$

Les nouvelles calculees pour les resultats sont:

$\begin{array}{llll}\text { Resultat 1 } & 0.35 & <0.96345488 & <0.95 \\ \text { Resultat 2 } & 0.35 & <0.83892672 & <0.95 \\ \text { Resultat 3 } & 0.4 & <0.83081793 & <0.9 \\ \text { Resultat 4 } & 0.3 & <0.83892189 & <1 \\ \text { Resultat 5 } & 0.3 & <0.86358963 & <1.0 \\ \text { Resultat 6 } & 0.3 & <0.83340909 & <1.0 \\ \text { Resultat 7 } & 0.3 & <0.83029164 & <0.8 \\ \text { Resultat 8 } & 0.3 & <0.99999899 & <0.9\end{array}$

resultat le plus compatible est le no $3(0.83081793)$

Les nouvelles normes calculees pour les programmes sont:

Programme 10.4

$<0.51428101$

$<1$

Programme 20.5

$<0.44999591$

$<0.8$

Programme 30.1

$<0.52739343$

$<0.8$

Programme 40.5

$<0.00014035$

$<0.8$

Programme 50.6

$<0.61639401$

$<0.8$

Programme 60.6

$<0.54427972$

$<0.8$

Programme 70.6

Programme 80.1

Programme 90.1

$<0.38529966$

$<0.76274103$

$<0.8$

$<0.76274103$

$<0.7$

Programme 10

Prooramme 11

$0.1<0.11298708$

$<0.7$

$0.1<0.11298708$

$<0.7$

$<0.7$ 


$\begin{array}{llll}\text { Programme 12 } & 0.2<0.64125249 & <0.6 \\ \text { Programme 13 } & 0.2<0.64202242 & <0.6 \\ \text { Programme 14 } & 0.2<0.57375621 & <0.6 \\ \text { Programme 15 } & 0.1<0.57375621 & <0.7\end{array}$

programme le plus compatible est le no 14(0.57375621)

Les nouvelles normes calculces pour les contextes sont:

$\begin{array}{lllll}\text { Contexte 1 } & 0.1 & <0.37682801 & <0.8 \\ \text { Contexte 2 } & 0.2 & <0.65047107 & <0.85 \\ \text { Contexte 3 } & 0.3 & <0.97768283 & <1.0 \\ \text { Contexte 4 } & 0.1 & <0.37682801 & <0.8 \\ \text { Contexte 5 } & 0.2 & <0.65047107 & <0.85 \\ \text { Contexte 6 } & 0.3 & <1.12067163 & <1.0 \\ \text { Contexte 7 } & 0.1 & <0.37624466 & <0.8 \\ \text { Contexte 8 } & 0.2 & <0.65047107 & <0.85 \\ \text { Contexte 9 } & 0.3 & <0.96977705 & <1.0\end{array}$

contexte le plus compatible est le no $3(0.97768283)$

Les nouvelles normes calculees pour les activites sont:

Activite $10.1<0.86487959<1$

Activite $20.4<0.8648802<1$

Activite $30.5<0.86488206<0.9$

Activite $40.5<0.86488206<0.9$

Activite $5 \quad 0.5<0.86488206<0.9$

Activite $60.5<0.86488206<0.9$

Activite $70.55<0.86488514<0.85$

Activite $80.55<0.86488514<0.85$

Activite $90.55<0.86488514<0.85$

Activite $100.55<0.86488514<0.85$

Activite $11 \quad 1.05<0.8600171<0.35$

Activite $120.55<0.86488514<0.85$

Activite $130.6<0.77840878<0.8$

Activite $140.6<0.77840878<0.8$

Activite $150.6<0.77840878<0.8$

Activite $160.6<0.77840878<0.8$

Activite $170.6<0.88790685<0.8$

Activite $181.1<0.81013159<0.3$

Activite $190.6<0.77842524<0.8$

Activite $20 \quad 0.6<0.7819673<0.8$

Activite $210.6<0.87265685<0.8$

Activite $220.4<0.34605059<0.7$

Activite $230.4<0.34605059<0.7$

Activite $240.4<0.34605059<0.7$

Activite $250.4<0.34605059<0.7$

Activite $260.9<0.35781915<0.2$

Activite $270.9<0.34835073<0.2$

Activite $280.4<0.60237133<0.7$

Activite $290.4<0.34605059<0.7$

Activite $30 \quad 0.7<0.88793448<0.3$

Activite $310.9<0.54043809<0.1$

Activite $320.9<0.54043809<0.1$

Activite $330.9<0.54053292<0.1$

activite le plus compatible est le no $4(0.86488206)$

La note est:1.0

La nouvelle norme pour le contexte choisi est: $0.97768283+(1.0-0.5)$

Les nouvelles calculees pour les resultats sont:

$\begin{array}{llll}\text { Resultat 1 } & 0.35 & <0.99759759 & <0.95 \\ \text { Resultat 2 } & 0.35 & <0.99004526 & <0.95 \\ \text { Resultat 3 } & 0.45 & <0.82835827 & <0.85 \\ \text { Resultat 4 } & 0.3 & <0.99004499 & <1 \\ \text { Resultat 5 } & 0.3 & <0.96296814 & <1.0 \\ \text { Resultat 6 } & 0.3 & <0.96118792 & <1.0\end{array}$


Resultat $7 \quad 0.3<0.82832297<0.8$

Resultat $8 \quad 0.3<0.99999993<0.9$

resultat le plus compatible est le no $4(0.99004499)$

Les nouvelles normes calculees pour les programmes sont:

$\begin{array}{llll}\text { Programme 10.4 } & <0.90651692 & <1 \\ \text { Programme 20.5 } & <0.49249049 & <0.8 \\ \text { Programme 30.1 } & <0.99998194 & <0.8 \\ \text { Programme 40.5 } & <0.00000099 & <0.8 \\ \text { Programme 50.6 } & <0.14219035 & <0.8 \\ \text { Programme 60.6 } & <0.21321997 & <0.8 \\ \text { Programme 70.6 } & <0.6514107<0.8 & \\ \text { Programme 80.1 } & <0.35534964 \quad<0.7 & \\ \text { Programme 90.1 } & <0.35534964 \quad<0.7 & \\ \text { Programme 10 } & 0.1 & <0.35530957 & <0.7 \\ \text { Programme 11 } & 0.1 & <0.35530957 & <0.7 \\ \text { Programme 12 } & 0.2 & <0.00453635 & <0.6 \\ \text { Programme 13 } & 0.2 & <0.0045418<0.6 & \\ \text { Programme 14 } & 0.3 & <0.78818378 & <0.5 \\ \text { Programme 15 } & 0.1 & <0.78818378 & <0.7\end{array}$

programme le plus compatible est le no $1(0.90651692)$

Les nouvelles normes calculees pour les contextes sont:

$\begin{array}{llll}\text { Contexte 1 } & 0.1 & <0.22249784 & <0.8 \\ \text { Contexte 2 } & 0.2 & <0.39473787 & <0.85 \\ \text { Contexte 3 } & 0.3 & <1.00348693 & <1.0 \\ \text { Contexte 4 } & 0.1 & <0.22249784 & <0.8 \\ \text { Contexte 5 } & 0.2 & <0.39473787 & <0.85 \\ \text { Contexte 6 } & 0.3 & <1.00075497 & <1.0 \\ \text { Contexte 7 } & 0.1 & <0.22249202 & <0.8 \\ \text { Contexte 8 } & 0.2 & <0.39473787 & <0.85 \\ \text { Contexte 9 } & 0.3 & <0.99982429 & <1.0\end{array}$

contexte le plus compatible est le no $9(0.99982429)$

Les nouvelles normes calculees pour les activites sont:

Activite $10.1<0.99939595<1$

Activite $20.4<0.74147387<1$

Aclivite $3 \quad 0.5<0.74147388<0.9$

Activite $4 \quad 1.0<0.74228784<0.4$

Activite $5 \quad 0.5<0.74147388<0.9$

Activite $60.5<0.74147388<0.9$

Activite $7 \quad 0.55<0.7414739<0.85$

Activite $8 \quad 0.55<0.7414739<0.85$

Activite $90.55<0.7414739<0.85$

Activite $10 \quad 0.55<0.7414739<0.85$

Activite $11 \quad 1.05<0.74144676<0.35$

Activite $120.55<0.7414739<0.85$

Activite $130.6<0.66732658<0.8$

Activite $140.6<0.66732658<0.8$

Activite $150.6<0.66732658<0.8$

Activite $16 \quad 0.6<0.66732658<0.8$

Activite $170.6<0.74160431<0.8$

Activite $18 \quad 1.1<0.66750752<0.3$

Activite $190.6<0.66732667<0.8$

Activite $20 \quad 0.6<0.66734652<0.8$

Activite $210.6<0.66788516<0.8$

Activite $22 \quad 0.4<0.2965901<0.7$

Activite $230.4<0.2965901<0.7$

Activite $24 \quad 0.4<0.2965901<0.7$

Activite $250.4<0.2965901<0.7$

Activite $260.9<0.29665702<0.2$

Activite $270.9<0.296603<0.2$

Anivits 20 ก A - n 2085470 - n 
Aclivite $290.4<0.2965901<0.7$

$\begin{array}{llll}\text { Activite } 30 & 0.7 & <0.74160446 & <0.3 \\ \text { Activite } 31 & 0.9 & <0.44500705 & <0.1\end{array}$

Aclivite $320.9<0.44500705<0.1$

Activite $330.9<0.4450076<0.1$

activite le plus compatible est le no $1(0.99939595)$

La note est:0.0

La nouvelle norme pour le contexte choisi est: $0.99982429+(0.0-0.5)$

Les nouvelles calculees pour les resultats sont:

$\begin{array}{llll}\text { Resultat 1 } & 0.35 & <0.99783789 & <0.95 \\ \text { Resultat 2 } & 0.35<0.99104439 & <0.95 \\ \text { Resultat 3 } & 0.45 & <0.84707332 & <0.85 \\ \text { Resultat 4 } & 0.35<0.99104415 & <0.95 \\ \text { Resultat 5 } & 0.3<0.96673199 & <1.0 \\ \text { Resultat 6 } & 0.3 & <0.96513604 & <1.0 \\ \text { Resultat 7 } & 0.3 & <0.84704226 & <0.8 \\ \text { Resultat 8 } & 0.3 & <0.99999994 & <0.9\end{array}$

resultat le plus compatible est le no $3(0.84707332)$

Les nouvelles normes calculees pour les programmes sont:

$\begin{array}{llll}\text { Programme 10.5 } & <0.87066018 & <0.9 \\ \text { Programme 20.5 } & <0.57384321 & <0.8 \\ \text { Programme 30.1 } & <0.83994012 & <0.8 \\ \text { Programme 40.5 } & <0.0000008<0.8 & \\ \text { Programme 50.6 } & <0.11458742 & <0.8 \\ \text { Programme 60.6 } & <0.44866836 & <0.8 \\ \text { Programme 7 0.6 } & <0.63386259 & <0.8 \\ \text { Programme 80.1 } & <0.28636683 & <0.7 \\ \text { Programme 90.1 } & <0.28636683 & <0.7 \\ \text { Programme 10 } & 0.1 & <0.28633454 & <0.7 \\ \text { Programme 11 } & 0.1 & <0.28633454 & <0.7 \\ \text { Programme 12 } & 0.2 & <0.59209914 & <0.6 \\ \text { Programme 13 } & 0.2 & <0.59209917 & <0.6 \\ \text { Programme 14 } & 0.3<0.82910139 & <0.5 \\ \text { Programme 15 } & 0.1 & <0.82910139 & <0.7\end{array}$

programme le plus compatible est le no 13(0.59209917)

Les nouvelles normes calculces pour les contextes sont:

$\begin{array}{llll}\text { Contexte 1 } & 0.1 & <0.3672388<0.8 \\ \text { Contexte 2 } & 0.2 & <0.62011983 & <0.85 \\ \text { Contexte 3 } & 0.3 & <0.95410059 & <1.0 \\ \text { Contexte 4 } & 0.1 & <0.3672388<0.8 \\ \text { Contexte 5 } & 0.2 & <0.62011983 & <0.85 \\ \text { Contexte 6 } & 0.3 & <0.95344762 & <1.0 \\ \text { Contexte 7 } & 0.1 & <0.367238<0.8 \\ \text { Contexte 8 } & 0.2 & <0.62011983 & <0.85 \\ \text { Contexte 9 } & 0.3 & <0.85910801 & <1.0\end{array}$

contexte le plus compatible est le no $3(0.95410059)$

Les nouvelles normes calculees pour les activites sont:

$\begin{array}{llll}\text { Activite 1 } & 0.3 & <0.78778038 & <1 \\ \text { Activite 2 } & 0.4 & <0.83533511 & <1 \\ \text { Activite 3 } & 0.5 & <0.83533511 & <0.9 \\ \text { Activite 4 } & 1.0 & <0.83543659 & <0.4 \\ \text { Activite 5 } & 0.5 & <0.83533511 & <0.9 \\ \text { Activite 6 } & 0.5 & <0.83533511 & <0.9 \\ \text { Activite 7 } & 0.55 & <0.83533511 & <0.85 \\ \text { Activite 8 } & 0.55<0.83533511 & <0.85 \\ \text { Activite 9 } & 0.55 & <0.83533511 & <0.85 \\ \text { Activite 10 } & 0.55 & <0.83533511 & <0.85 \\ \text { Activite 11 } & 1.05 & <0.83533173 & <0.35 \\ \text { Activite 12 } & 0.55<0.83533511 & <0.85 \\ \text { Artivite 12 } & 06 & <075180161 & -00\end{array}$


$\begin{array}{lllll}\text { Activite 14 } & 0.6 & <0.75180161 & <0.8 \\ \text { Activite 15 } & 0.6 & <0.75180161 & <0.8 \\ \text { Activite 16 } & 0.6 & <0.75180161 & <0.8 \\ \text { Activite 17 } & 0.6 & <0.85793534 & <0.8 \\ \text { Activite 18 } & 1.1 & <0.75182416 & <0.3 \\ \text { Activite 19 } & 0.6 & <0.75180162 & <0.8 \\ \text { Activite 20 } & 0.6 & <0.84745145 & <0.8 \\ \text { Activite 21 } & 0.6 & <0.75187124 & <0.8 \\ \text { Activite 22 } & 0.4 & <0.33413411 & <0.7 \\ \text { Activite 23 } & 0.4 & <0.33413411 & <0.7 \\ \text { Activite 24 } & 0.4 & <0.33413411 & <0.7 \\ \text { Activite 25 } & 0.4 & <0.33413411 & <0.7 \\ \text { Activite 26 } & 0.9 & <0.33414245 & <0.2 \\ \text { Activite 27 } & 0.9 & <0.5921603< & 0.2 \\ \text { Activite 28 } & 0.4 & <0.33437881 & <0.7 \\ \text { Activite 29 } & 0.4 & <0.33413411 & <0.7 \\ \text { Activite 30 } & 0.7 & <0.85793536 & <0.3 \\ \text { Activite 31 } & 0.9 & <0.52223413 & <0.1 \\ \text { Activite 32 } & 0.9 & <0.52223413 & <0.1 \\ \text { Activite 33 } 0.9 & <0.52223419 & <0.1\end{array}$

activite le plus compatible est le no $10(0.83533511)$

La note est:1.0

La nouvelle norme pour le contexte choisi est: $0.95410059+(1.0-0.5)$

Les nouvelles calculees pour les resultats sont:

$\begin{array}{llll}\text { Resultat 1 } & 0.35 & <0.83581266 & <0.95 \\ \text { Resultat 2 } & 0.35 & <0.9994462<0.95 \\ \text { Resultat 3 } & 0.5 & <0.99119073 & <0.8 \\ \text { Resultat 4 } & 0.35<0.83531056 & <0.95 \\ \text { Resultat 5 } & 0.3 & <0.94208454 & <1.0 \\ \text { Resultat 6 } & 0.3 & <0.89610831 & <1.0 \\ \text { Resultat 7 } & 0.3 & <0.82541313 & <0.8 \\ \text { Resultat 8 } & 0.3 & <1.0<0.9 & \end{array}$

resultat le plus compatible est le no $5(0.94208454)$

Les nouvelles normes calculces pour les programmes sont:

\begin{tabular}{|c|c|c|c|c|}
\hline rogramme 10.5 & $<$ & 4931067 & 0.9 & \\
\hline Programme 20.5 & $<$ & 2416886 & 0.8 & \\
\hline Programme 30.1 & $<$ & 9949419 & 0.8 & \\
\hline Programme 40.5 & $<$ & 0000003 & 0.8 & \\
\hline Programme 50.6 & $<$ & \multicolumn{3}{|c|}{$0.6543209<0.8$} \\
\hline Programme 60.6 & \multicolumn{2}{|c|}{0.77311269} & 0.8 & \\
\hline Programme 70.6 & $<$ & 0.54945434 & 0.8 & \\
\hline Programme 80.1 & $<$ & 0.20588557 & 0.7 & \\
\hline Programme 90.1 & $<$ & 0.20588557 & 0.7 & \\
\hline Programme 10 & 0.1 & $<0.61713535$ & & $<0.7$ \\
\hline Programme 11 & 0.1 & $<0.61713535$ & & $<0.7$ \\
\hline Programme 12 & 0.2 & $<0.68646765$ & & $<0.6$ \\
\hline rogramme 13 & 0.3 & $<0.68646765$ & & $<0.5$ \\
\hline Programme 14 & 0.3 & $<0.2084773$ & 0.5 & \\
\hline Programme 15 & 0.1 & $<0.2084773$ & 0.7 & \\
\hline
\end{tabular}

programme le plus compatible est le no $6(0.77311269)$

Les nouvelles normes calculees pour les contextes sont:

$\begin{array}{llll}\text { Contexte 1 } & 0.1 & <0.25296671 & <0.8 \\ \text { Contexte 2 } & 0.2 & <0.5956447<0.85 \\ \text { Contexte 3 } & 0.3 & <1.02845003 & <1.0 \\ \text { Contexte 4 } & 0.1 & <0.25296671 & <0.8 \\ \text { Contexte 5 } & 0.2 & <0.5956447<0.85 \\ \text { Contexte 6 } & 0.3 & <0.99764263 \quad<1.0 \\ \text { Contexte 7 } & 0.1 & <0.25296665 \quad<0 \\ \text { Contexte 8 } & 0.2 & <0.5956447<0.85 \\ \text { Contexte 9 } & 0.3 & <0.99319475 \quad<1.0\end{array}$


contexte le plus compatible est le no $6(0.99764263)$

Les nouvelles normes calculees pour les activites sont:

Activite $20.4<0.7919114<1$

Activite $30.5<0.82779201<0.9$

Activite $4 \quad 1.0<0.79191482<0.4$

Activite $5 \quad 0.5<0.7919114<0.9$

Activite $6 \quad 0.5<0.7919114<0.9$

Activite $7 \quad 0.55<0.7919114<0.85$

Activite $8 \quad 0.55<0.92713938<0.85$

Activite $9 \quad 0.55<0.7919114<0.85$

Activite $101.05<0.93232995<0.35$

Activite $11 \quad 1.05<0.79191129<0.35$

Activite $120.55<0.7919114<0.85$

Activite $130.6<0.71272026<0.8$

Activite $140.6<0.71272026<0.8$

Activite $150.6<0.93337816<0.8$

Activite $160.6<0.71272026<0.8$

Activite $170.6<0.87126729<0.8$

Activite $181.1<0.71272102<0.3$

Activite $190.6<0.71272026<0.8$

Activite $200.6<0.86331872<0.8$

Activite $210.6<0.71272261<0.8$

Activite $220.4<0.31676456<0.7$

Activite $230.4<0.31676456<0.7$

Activite $240.4<0.31676456<0.7$

Activite $250.4<0.31676456<0.7$

Activite $260.9<0.48072947<0.2$

Activite $270.9<0.48860388<0.2$

Activite $280.4<0.31677281<0.7$

Activite $290.4<0.31676456<0.7$

Activite $30 \quad 0.7<0.79268268<0.3$

Activite $310.9<0.47586972<0.1$

Activite $320.9<0.47586972<0.1$

Activite $330.9<0.47586972<0.1$

activite le plus compatible est le no $3(0.82779201)$

La note est:1.0

La nouvelle norme pour le contexte choisi est: $0.99764263+(1.0-0.5)$

Les nouvelles calculees pour les resultats sont:

$\begin{array}{llll}\text { Resultat 1 } & 0.35 & <0.98924981 & <0.95 \\ \text { Resultat 2 } & 0.35<0.99996072 & <0.95 \\ \text { Resultat 3 } & 0.5 & <0.99937756 & <0.8 \\ \text { Resultat 4 } & 0.35<0.83190772 & <0.95 \\ \text { Resultat 5 } & 0.3<0.96829121 & <1.0 \\ \text { Resultat 6 } & 0.3<0.96519007 & <1.0 \\ \text { Resultat 7 } & 0.3 & <0.83120653 & <0.8 \\ \text { Resultat 8 } & 0.3 & <1.0<0.9 & \end{array}$

resultat le plus compatible est le no 5(0.96829121)

Les nouvelles normes calculees pour les programmes sont:

Programme $10.5<0.90858474<0.9$

Programme $20.5<0.00055042<0.8$

Programme $30.1<0.99995049<0.8$

Programme 40.5 $<7.67478550$ E-10 $<0.8$

Programme $50.6<0.46985007<0.8$

Programme $60.7<0.50690797<0.7$

Programme $70.6<0.41745443<0.8$

Programme 80.1 $<0.41534248<0.7$

Programme $90.1<0.41534248<0.7$

Programme $10 \quad 0.1<0.41555376<0.7$

Programme $11 \quad 0.1<0.41555376<0.7$ 


$\begin{array}{llll}\text { Programme 12 } & 0.2<0.41561015 & <0.6 \\ \text { Programme 13 } & 0.3 & <0.41561015 & <0.5 \\ \text { Programme 14 } & 0.3<0.20868744 & <0.5 \\ \text { Programme 15 } & 0.1 & <0.20868744 & <0.7\end{array}$

programme le plus compatible est le no 13(0.41561015)

Les nouvelles normes calculees pour les contextes sont:

$\begin{array}{llll}\text { Contexte 1 } & 0.1 & <0.24499933 & <0.8 \\ \text { Contexte 2 } & 0.2 & <0.51504934 & <0.85 \\ \text { Contexte 3 } & 0.3 & <0.98639027 & <1.0 \\ \text { Contexte 4 } & 0.1 & <0.24499933 & <0.8 \\ \text { Contexte 5 } & 0.2 & <0.51504934 & <0.85 \\ \text { Contexte 6 } & 0.3 & <1.12912463 & <1.0 \\ \text { Contexte 7 } & 0.1 & <0.24499931 & <0.8 \\ \text { Contexte 8 } & 0.2 & <0.51504934 & <0.85 \\ \text { Contexte 9 } & 0.3 & <0.97714254 & <1.0\end{array}$

contexte le plus compatible est le no $3(0.98639027)$

Les nouvelles normes calculees pour les activites sont:

$\begin{array}{llll}\text { Activite 1 } & 0.3 & <0.89208327 & <1 \\ \text { Activite 2 } & 0.4 & <0.89241187 & <1 \\ \text { Activite 3 } & 1.0 & <0.94079665 & <0.4 \\ \text { Activite 4 } & 1.0 & <0.89241259 & <0.4 \\ \text { Activite 5 } & 0.5 & <0.89241187 & <0.9 \\ \text { Activite 6 } & 0.5 & <0.89241187 & <0.9 \\ \text { Activite 7 } & 0.55 & <0.89241187 & <0.85 \\ \text { Activite 8 } & 0.55 & <0.9228744 & 0.85 \\ \text { Activite 9 } & 0.55 & <0.89241187 & <0.85 \\ \text { Activite 10 } & 1.05 & <0.92411747 & <0.35 \\ \text { Activite 11 } & 1.05 & <0.89241185 & <0.35 \\ \text { Activite 12 } & 0.55 & <0.89241187 & <0.85 \\ \text { Activite 13 } & 0.6 & <0.80317068 & <0.8 \\ \text { Activite 14 } & 0.6 & <0.80317068 & <0.8 \\ \text { Activite 15 } & 0.6 & <0.85526989 & <0.8 \\ \text { Activite 16 } & 0.6 & <0.80317068 & <0.8 \\ \text { Activite 17 } & 0.6 & <0.91987998 & <0.8 \\ \text { Activite 18 } & 1.1 & <0.80317084 & <0.3 \\ \text { Activite 19 } & 0.6 & <0.80317068 & <0.8 \\ \text { Activite 20 } & 0.6 & <0.88038658 & <0.8 \\ \text { Activite 21 } & 0.6 & <0.80317118 & <0.8 \\ \text { Activite 22 } & 0.4 & <0.35696475 & <0.7 \\ \text { Activite 23 } & 0.4 & <0.35696475 & <0.7 \\ \text { Activite 24 } & 0.4 & <0.35696475 & <0.7 \\ \text { Activite 25 } & 0.4 & <0.35696475 & <0.7 \\ \text { Activite 26 } & 0.9 & <0.39815715 & <0.2 \\ \text { Activite 27 } & 0.9 & <0.52463939 & <0.2 \\ \text { Activite 28 } & 0.4 & <0.35696649 & <0.7 \\ \text { Activite 29 } & 0.4 & <0.35696475 & <0.7 \\ \text { Activite 30 } & 0.7 & <0.90281445 & <0.3 \\ \text { Activite 31 } & 0.9 & <0.54516814 & <0.1 \\ \text { Activite 32 } & 0.9 & <0.54516814 & <0.1 \\ \text { Activite 33 } & 0.9 & <0.54516814 & <0.1\end{array}$

activite le plus compatible est le no $6(0.89241187)$

La note est:0.4

La nouvelle norme pour le contexte choisi est: $0.98639027+(0.4-0.5)$

Les nouvelles calculees pour les resultats sont:

$\begin{array}{llll}\text { Resultat 1 } & 0.35 & <0.93943809 & <0.95 \\ \text { Resultat 2 } & 0.35 & <0.99998625 & <0.95 \\ \text { Resultat 3 } & 0.5 & <0.99978218 & <0.8 \\ \text { Resultat 4 } & 0.35<0.94457481 & <0.95 \\ \text { Resultat 5 } & 0.3< & <0.97839696 & <1.0 \\ \text { Resultat 6 } & 0.3<0.97732387 & <1.0\end{array}$


Resultat $7 \quad 0.3<0.88421843<0.8$

Resultat $8 \quad 0.3<1.0<0.9$

resultat le plus compatible est le no $4(0.94457481)$

Les nouvelles normes calculees pour les programmes sont:

Programme $10.5<0.85595357<0.9$

Programme $20.5<0.54317418<0.8$

Programme $30.1<0.99998565<0.8$

Programme 40.5 $<4.13232078 \mathrm{E}-10<0.8$

Programme $50.6<0.25510568<0.8$

Programme $60.7<0.29019063<0.7$

Programme $70.6<0.62002488<0.8$

Programme 80.1 $<0.2236321<0.7$

Programme $90.1<0.2236321<0.7$

Programme $10 \quad 0.1<0.27759023<0.7$

Programme $11 \quad 0.1<0.27759023<0.7$

Programme $12 \quad 0.2<0.2776147<0.6$

Programme $13 \quad 0.4<0.2776147<0.4$

Programme $14 \quad 0.3<0.80108564<0.5$

Programme $15 \quad 0.1<0.80108564<0.7$

programme le plus compatible est le no $1(0.85595357)$

Les nouvelles normes calculees pour les contextes sont:

$\begin{array}{llll}\text { Contexte 1 } & 0.1 & <0.2228734<0.8 \\ \text { Contexte 2 } & 0.2 & <0.39741604 & <0.85 \\ \text { Contexte 3 } & 0.3 & <0.99743546 & <1.0 \\ \text { Contexte 4 } & 0.1 & <0.2228734<0.8 \\ \text { Contexte 5 } & 0.2<0.39741604 & <0.85 \\ \text { Contexte 6 } & 0.3 & <1.00328022 & <1.0 \\ \text { Contexte 7 } & 0.1 & <0.2228734<0.8 \\ \text { Contexte 8 } & 0.2 & <0.39741604 & <0.85 \\ \text { Contexte 9 } & 0.3 & <0.99945976 & <1.0\end{array}$

contexte le plus compatible est le no 9(0.99945976)

Les nouvelles normes calculces pour les activites sont:

Activite $10.3<0.99878138<1$

Activite $20.4<0.7612511<1$

Activite $3 \quad 1.0<0.76194575<0.4$

Activite $4 \quad 1.0<0.76125111<0.4$

Activite $5 \quad 0.5<0.7612511<0.9$

Activite $6 \quad 1.0<0.75624841<0.4$

Activite $7 \quad 0.55<0.7612511<0.85$

Activite $80.55<0.76168424<0.85$

Activite $9 \quad 0.55<0.7612511<0.85$

Activite $101.05<0.76170222<0.35$

Activite $11 \quad 1.05<0.7612511<0.35$

Activite $120.55<0.7612511<0.85$

Activite $130.6<0.68512599<0.8$

Activite $140.6<0.68512599<0.8$

Activite $150.6<0.68587778<0.8$

Activite $160.6<0.68512599<0.8$

Activite $170.6<0.76164103<0.8$

Activite $181.1<0.68512599<0.3$

Activite $190.6<0.68512599<0.8$

Activite $200.6<0.68625677<0.8$

Activite $210.6<0.685126<0.8$

Activite $220.4<0.30450044<0.7$

Activite $230.4<0.30450044<0.7$

Aclivite $240.4<0.30450044<0.7$

Activite $250.4<0.30450044<0.7$

Activite $260.9<0.30510911<0.2$

Activite $270.9<0.30738232<0.2$

Activite $28 \quad 0.4<0.30450046<0.7$ 


$\begin{array}{llll}\text { Activite 29 } & 0.4 & <0.30450044 & <0.7 \\ \text { Activite 30 } & 0.7 & <0.76139741 & <0.3 \\ \text { Activite 31 } & 0.9 & <0.45688781 & <0.1 \\ \text { Activite 32 } & 0.9 & <0.45688781 & <0.1 \\ \text { Activite 33 } & 0.9 & <0.45688781 & <0.1\end{array}$

activite le plus compatible est le no $1(0.99878138)$

La note est:0.0

La nouvelle norme pour le contexte choisi est: $0.99945976+(0.0-0.5)$

Les nouvelles calculees pour les resultats sont:

$\begin{array}{llll}\text { Resultat 1 } & 0.35 & <0.94565273 & <0.95 \\ \text { Resultat 2 } & 0.35<0.99998762 & <0.95 \\ \text { Resultat 3 } & 0.5 & <0.99980391 & <0.8 \\ \text { Resultat 4 } & 0.4<0.95024812 & <0.9 \\ \text { Resultat 5 } & 0.3<0.98057303 & <1.0 \\ \text { Resultat 6 } & 0.3<0.97960917 & <1.0 \\ \text { Resultat 7 } & 0.3<0.89643822 & <0.8 \\ \text { Resultat 8 } & 0.3<0<0.9 & \end{array}$

resultat le plus compatible est le no $1(0.94565273)$

Les nouvelles normes calculees pour les programmes sont:

\begin{tabular}{|c|c|c|c|c|c|}
\hline Programme 10.6 & $<$ & 0.83147402 & $<$ & 0.8 & \\
\hline Programme 20.5 & $<$ & $0.6180248<0.8$ & & & \\
\hline Programme 30.1 & $<$ & 0.79088644 & $<$ & 0.8 & \\
\hline Programme 40.5 & $<$ & $3.07625463 E-10$ & - & 0.8 & \\
\hline Programme 50.6 & $<$ & 0.50449397 & $<$ & 0.8 & \\
\hline Programme 60.7 & $<$ & 0.45514947 & $<$ & 0.7 & \\
\hline Programme 70.6 & $<$ & 0.46156979 & $<$ & 0.8 & \\
\hline Programme 80.1 & $<$ & 0.68813401 & $<$ & 0.7 & \\
\hline Programme 90.1 & $<$ & 0.68813401 & $<$ & 0.7 & \\
\hline Programme 10 & 0.1 & $<0.63546203$ & & & $<0.7$ \\
\hline Programme 11 & 0.1 & $<0.63546203$ & & & $<0.7$ \\
\hline Programme 12 & 0.2 & $<0.2066668$ & & 0.6 & \\
\hline Programme 13 & 0.4 & $<0.2066668$ & & 0.4 & \\
\hline Programme 14 & 0.3 & $<0.5963582$ & & 0.5 & \\
\hline Programme 15 & 0.1 & $<0.5963582$ & & 0.7 & \\
\hline
\end{tabular}

programme le plus compatible est le no $3(0.79088644)$

Les nouvelles normes calculces pour les contextes sont:

$\begin{array}{llll}\text { Contexte 1 } & 0.1 & <0.4900745<0.8 \\ \text { Contexte 2 } & 0.2 & <0.71325736 \quad<0.85 \\ \text { Contexte 3 } & 0.3 & <0.99988812<0 \\ \text { Contexte 4 } & 0.1 & <0.4900745<0.8 \\ \text { Contexte 5 } & 0.2 & <0.71325736 \quad<0 \\ \text { Contexte 6 } & 0.3 & <1.0001435<1.0 \\ \text { Contexte 7 } & 0.1 & <0.4900745<0.8 \\ \text { Contexte 8 } & 0.2 & <0.71325736 & \\ \text { Contexte 9 } & 0.3 & <0.98347244 & <0.85 \\ \text { Conte }\end{array}$

contexte le plus compatible est le no $3(0.99988812)$

Les nouvelles normes calculees pour les activites sont:

Aclivite $10.5<0.77395616<1$

Activite $20.4<0.9944704<1$

Activite $3 \quad 1.0<0.92416665<0.4$

Activite $4 \quad 1.0<0.92415163<0.4$

Activite $50.5<0.92415163<0.9$ s

Activite $6 \quad 1.0<0.9240439<0.4$

Aclivite $70.55<0.86551849<0.85$

Activite $80.55<0.86552848 \quad<0.85$

Activite $90.55<0.86551849<0.85$

Activite $101.05<0.8655289<0.35$

Aclivite $11 \quad 1.05<0.86551849<<0.35$

Activite $120.55<0.86551849<0.85$

Activite $130.6<0.74663917<0.8$ 
Activite 14

Activite 15

0.6

$<0.74663917$

$<0.8$

Activite 16 .

0.6

$<0.74665727$

$<0.8$

-

Activite 17

$0.6<0.74663917$

$<0.8$

Activite 18

$0.6<0.99447823$

. $<0.8$

Activite 19

1.1

$<0.85869487$

$<0.3$

Activite 20

$0.6<0.85869487$

$<0.8$

Activite 21

0.6

$<0.85871856$

$<0.8$

Activite 22

$0.6<0.85869488$

$<0.8$

Activite 23

$0.4<0.4381372<0.7$

Activite 24

$0.4<0.4381372<0.7$

Activite 25

$0.4<0.4381372<0.7$

Activite 26

$0.4<0.4381372<0.7$

Activite 27

$0.9<0.4381483<0.2$

Activite 28

Activite 29

$0.9<0.43818997<0.2$

Activite 30

$0.4<0.4381372<0.7$

Activite 31

$0.4<0.4381372<0.7$

Activite 32

$0.7<0.7837174<0.3$

$0.9<0.4702317<0.1$

$<0.4702317<0.1$

Activite $330.9<0.4702317<0.1$

activite le plus compatible est le no $2(0.9944704)$

La note est:7.0

La nouvelle norme pour le contexte choisi est: $0.99988812+(7.0-0.5)$

Les nouvelles calculees pour les resultats sont:

$\begin{array}{lllr}\text { Resultat 1 } & 0.4 & <0.96789376 & <0.9 \\ \text { Resultat 2 } & 0.35 & <0.9999926<0.95 \\ \text { Resultat 3 } & 0.5 & <0.99988284 & <0.8 \\ \text { Resultat 4 } & 0.4 & <0.97057927 & <0.9 \\ \text { Resultat 5 } & 0.3 & <0.98843803 & <1.0 \\ \text { Resultat 6 } & 0.3 & <0.98786681 & <1.0 \\ \text { Resultat 7 } & 0.3 & <0.83382579 & <0.8 \\ \text { Resultat 8 } & 0.3 & <1.0<0.9 & \end{array}$

Resultat $80.3<1.0<0.9$

resultat le plus compatible est le no $5(0.98843803)$

Les nouvelles normes calculces pour les programmes sont:

Programme $10.6<0.8132973<0.8$

Programme $20.5<0.60421409<0.8$

Programme $30.2<0.91844582<0.7$

Programme $40.5<0.30221584<0.8$

Programme 50.6 $<0.56207681<0.8$

Programme $60.7<0.54419413<0.7$

Programme 70.6 $<0.54644866<0.8$

Programme $80.1<0.50022966<0.7$

Programme 90.1 $<0.50022966<0.7$

Programme $10 \quad 0.1<0.47021668<0.7$

Programme $11 \quad 0.1<0.47021668<0.7$

Programme $12 \quad 0.2<0.26503541<0.6$

Programme 13 ' $0.4<0.26503541<0.4$

Programme $14 \quad 0.3<0.44830774<0.5$

Programme $15 \quad 0.1<0.44830774<0.7$

programme le plus compatible est le no 14(0.44830774)

Les nouvelles normes calculees pour les contextes sont:

Contexte $1 \quad 0.1<0.28717929<0.8$

Contexte $20.2<0.52758853<0.85$

Contexte $30.3<3.56326235<1.0$

Contexte $40.1<0.28717929<0.8$

Contexte $50.2<0.52758853<0.85$

Contexte $60.3<0.98717906<1.0$

Contexte $70.1<0.28717929<0.8$

Contexte $8 \quad 0.2<0.52758853<0.85$

Contexte $90.3<0.98361885<1.0$ 
contexte le plus compatible est le no 6(0.98717906)

Les nouvelles normes calculees pour les activites sont:

Activite $1 \quad 0.5<0.8831583<1$

Activite $20.9<3.33209158<0.5$

Activite $3 \quad 1.0<0.91343892<0.4$

Activite $4 \quad 1.0<0.91343568<0.4$

Activite $50.5<0.91343568<0.9$

Activite $6 \quad 1.0<0.91341243<0.4$

Activite $7 \quad 1.05<0.9011019<0.35$

Activite $8 \quad 1.05<0.90110394<0.35$

Activite $9 \quad 1.05<0.9011019<0.35$

Activite $101.55<0.90110403<-0.15$

Activite $11 \quad 1.55<0.9011019<-0.15$

Activite $121.05<0.9011019<0.35$

Activite $131.1<0.80448051<0.3$

Activite $141.1<0.80448051<0.3$

Activite $151.1<0.8044841<0.3$

Activite $161.1<0.80448051<0.3$

Activite $170.6<0.94056572<0.8$

Activite $181.1<0.82798237<0.3$

Activite $190.6<0.82798237<0.8$

Activite $20 \quad 0.6<0.8279876<0.8$

Activite $210.6<0.87843062<0.8$

Activite $220.4<0.38117696<0.7$

Activite $230.4<0.38117696<0.7$

Activite $240.4<0.38117696<0.7$

Activite $250.4<0.38117696<0.7$

Activite $260.9<0.38117968<0.2$

Activite $270.9<0.3811899<0.2$

Activite $280.4<0.52896554<0.7$

Activite $290.4<0.38117696<0.7$

Activite $30 \quad 0.7<0.89706902<0.3$

Activite $310.9<0.54227498<0.1$

Activite $320.9<0.54227498<0.1$

Activite $330.9<0.54227498<0.1$

activite le plus compatible est le no $1(0.8831583)$

La note est: 1.0

La nouvelle norme pour le contexte choisi est: $0.98717906+(1.0-0.5)$

Les nouvelles calculees pour les resultats sont:

$\begin{array}{llll}\text { Resultat 1 } & 0.4 & <0.84006956 & <0.9 \\ \text { Resultat 2 } & 0.35 & <0.84265275 & <0.95 \\ \text { Resultat 3 } & 0.5 & <0.84264378 & <0.8 \\ \text { Resultat 4 } & 0.4 & <0.84028275 & <0.9 \\ \text { Resultat 5 } & 0.3<0.84171409 & <1.0 \\ \text { Resultat 6 } & 0.3<0.84166794 & <1.0 \\ \text { Resultat 7 } & 0.3 & <0.98945941 & <0.8 \\ \text { Resultat 8 } & 0.3 & <0.84265335 & <0.9\end{array}$

resultat le plus compatible est le no $8(0.84265335)$

Reussite de l'objectif

Les resultats obtenus sont:

Resultat no 1: 0.84006956

Resultat no 2: 0.84265275

Resultat no 3: 0.84264378

Resultat no 4: 0.84028275

Resultat no 5: 0.84171409

Resultat no 6: 0.84166794

Resultat no 7: 0.98945941

Resultat no 8: 0.84265335 